九州大学学術情報リポジトリ

Kyushu University Institutional Repository

\title{
Palaeontological Study of the Miyazaki Group : A General Account of Faunas
}

Shuto, Tsugio

Faculty of Sciences, Kyushu University

https://doi.org/10.5109/1526109

出版情報：九州大學理學部紀要：Series D, Geology. 10 (2)，pp.73-206，1961-03-10. Faculty of Science, Kyushu University バージョン :

権利関係 : 
Mem. Fac. Sci., Kyushu Univ., Ser. D, Geology, Vol. X, No. 2, pp. 73-206, text-figs. 1-23, tables 1-5, pls. 11-13, March 10, 1961

\title{
Palaeontological Study of the Miyazaki Group - A General Account of the Faunas -
}

By

\section{Tsugio SHUTO}

\begin{abstract}
This report contains three principal results of the palaeontological study of the Miyazaki group. They concern with palaeoecology, biostratigraphical correlation, and evolution problem.

The Miyazaki group, occupying the south-east part of the Miyazaki Prefecture, Japan, includes two major cycles of sedimentation and is composed of laterally segregated a few sedimentary facies.

The faunal and the lineal successions of the mollusks of the Miyazaki group are surprisingly concordant each other and a few distinct faunal zonules and topozones are recognized. The results of the correlation depends much upon the materials based on and they are not always quite harmonious one another according to the different groups of the organisms. My conclusion on age, which is harmonized to the data on the mollusks, higher foraminifers, and plankton foraminifers with least inconsistence, indicates that the Miyazaki group ranges from upper Helvetian to Plaisancian.

Salinity and water temperature are respectively one of the most important limiting factors for the neritic benthos. Temperature of the sea water is considered to have lowered down gradually from tropic to subtropic condition in the laspe of time from late Helvetian to Tortonian. Thereafter no essential change took place. While the area was differentiated into several physiological environments including open oceanic one and bay water one and the biofacies apparently correspond to these environments at the beginning of the first transgression. Then the oceanographic conditions became gradually more open oceanic uniformly over the whole area and the minor segregations of the molluscan communities seems to be due to the mutual reaction among organisms.

Concerning the evolution of the ancient animals recognition of the speciation process is very important, but it seems to have been neglected by palaeontologists for long period. The approach to this problem, however, may be possible by means of the analysis of the intraspecies polytypy, especially the sympatric subspecies and of the examination of the relation between these subspecies and of the examination of the relation between these subspecies and the environmental factors of their habitats. Paphia (Paphia) exilis SHuto and a few other examples are offered here.
\end{abstract}

\footnotetext{
* Received September 14, 1960.
} 


\section{Contents}

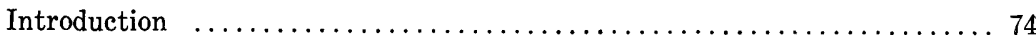

Acknowledgements $\ldots \ldots \ldots \ldots \ldots \ldots \ldots \ldots \ldots \ldots \ldots \ldots \ldots \ldots 77$

A brief note on the lithostratigraphy of the Miyazaki group......... 78

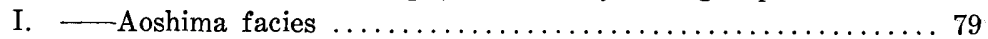

II. -Tsuma facies $\ldots \ldots \ldots \ldots \ldots \ldots \ldots \ldots \ldots \ldots \ldots \ldots \ldots 1$

III. - Miyazaki facies $\ldots \ldots \ldots \ldots \ldots \ldots \ldots \ldots \ldots \ldots \ldots \ldots \ldots \ldots$

IV. - Sedimentary history of the Miyazaki group .......... 85

Complete list of the molluscan species from the Miyazaki group ...... 87

Palaeoecology of the molluscan faunas $\ldots \ldots \ldots \ldots \ldots \ldots \ldots \ldots \ldots . \ldots 9$

I Descriptions of the localities and the fossil assemblages .... 98

II. - Stratigraphic position of the fossil localities . . . . . . . . . . 117

III. - Physico-chemical aspects of the fossil beds of the first

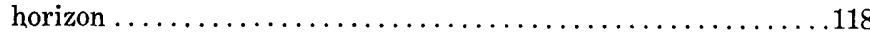

IV. - Biologic aspects of the fossil beds of the first horizon . . . . 122

V. Biologic features of the fossil beds of the second to the

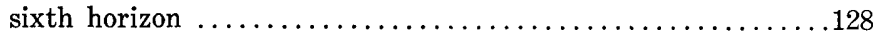

VI. Concluding remarks on the palaeoecology ............131

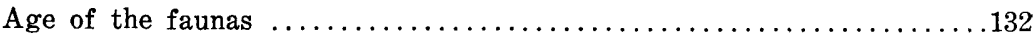

I. - Analysis of the fossil assemblages ...................132

II. - Biologic sequence and the zonation in the Miyazaki group ..150

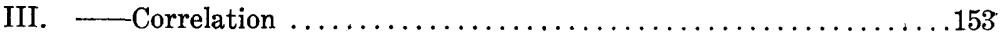

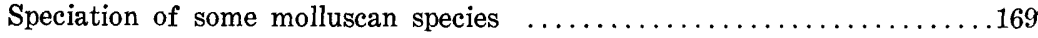

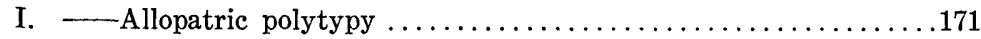

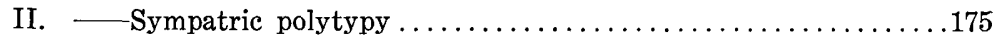

III. Environmental influence to the speciation of the marine mollusca .................................. 180

IV. - Paphia exilis group and its characteristics .............185

V. Adaptive evolution of Venericardia (Megacardita) panda

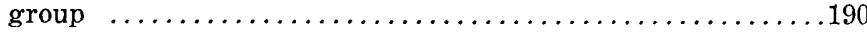

VI. - Other examples of sympatric species among the marine

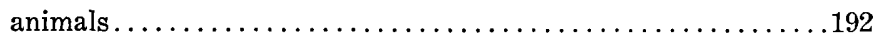

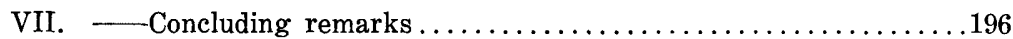

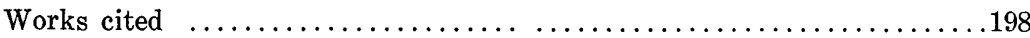

\section{Introduction}

This report is the results of the general considerations of the fossil assemblages of the Miyazaki group. This completes the "Palaeontological Study of the Miyazaki group" together with the descriptive parts, some of which have been issued serially since 1955, and others are being prepared for future publication.

The late Professor M. Yoкоуама (1928, 331-350) was the first who published the result of purely palaeontological study on the material from the Miyazaki group. Of course some authors, such as S. OTSUKA $(1900,14-15)$ and T. IGI $(1904,19-21)$, listed a few molluscan species in their papers on the general geology of the Miyazaki district. However they neither mentioned the analytic or synthetic results of the fossil assemblages in detail, nor attempted to do so. 
In 1928 YoKoyama described 218 molluuscan species in the paper entitled "Pliocene Shells from Hyuga" based on the T. KoBAyashi's collection from the northern localities of the Miyazaki group. He stratigraphically divided the group into four "Zones" (Zones A to D) and determined the geologic age of each "Zone" on the basis of the molluscan assemblages included in it. He adopted the Lyellian method on that occasion, as he had already tried at the several younger Caenozoic faunas from various localities of our country. According to him the assemblage from the uppermost horizon of the Miyazaki group, Zone A, contains the living species by about three-fourths of the total number of the species and shows the faunal affinity to the Lower Musashino and to other Pliocene faunas in our country. Thereupon he concluded that the age of Zone $\mathrm{A}$ was Lower Musashino or Upper Pliocene. In the similar manner he determined the age of Zones B, C, and D as Middle Pliocene, upper Lower Pliocene, and Lower Pliocene, respectively.

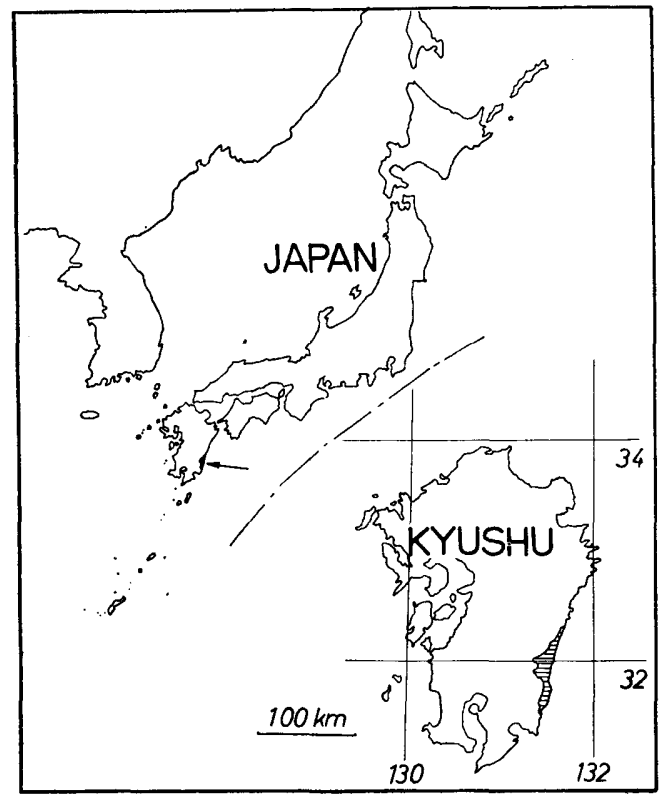

Text-fig. 1. Index map of the Miyazaki group.

Although his contributions to the biochronology are very valuable, the conclusions do not necessarily agree to the recent knowledge on the Neogene biostratigraphy in our country, and his classification of the fossil material includes some doubtful or incorrect points in the sense of the modern taxonomy. Furthermore his material was obtained from so limited small part of the Miyazaki group that it is not sufficient enough to argue the biostratigraphy of the whole group. Hereupon some revisions on the faunal analysis with additional data from various parts of the Miyazaki group are now necessary.

Y. OTUKA (1930, 1048-1074) offered a refined stratigraphy of the Miyazaki 
group of almost the same area as that YokoYama once treated. He stated that the strata developed in the suburbs of Tsuma and Takanabe Town were divided into two parts with a major unconformity. The lower part consists chiefly of the grey massive mudstones and was called by him the Tsuma group, while the upper part consists of the alternations of sandstones and siltstones of light grey color and was named the Takanabe group. He determined further the geologic ages of these groups on the basis of the molluscan fossils. According to him they are Upper Miocene and Plaisancian in age, respectively. Concerning the geologic age my conclusion is not much different from his result, but is remarkably divergent from his opinion about the geologic relation between the two. The detailed field survey has clarified that a thin but distinct tuff bed lies continuously across the lithological boundary which he considered the unconformity. He seems to have been misled by rather the abrupt change in the lithofacies from the monotonous mudstones of his "Tsuma group" to the thick medium sandstones of the lower part of his "Takanabe group".

Although T. UcHro (1947, 87-88) scrutinized OTUKA's frame work of the local stratigraphy in the area surrounding Miyazaki City, he also erroneously reccgnized the unconformity between the lower and the upper part, that is to say, between his "Miyazaki group" and the "Uryuno formation", which he correlated to the Tsuma and to the Takanabe group, respectively. He furthermore referred to the fossil remains, especially to the significance of Operculina complanata japonica HANZAWA and $O$. ammonoides (GRoNovius).

J. IToIgawa (1953, 213-218) described a new species of Siphonalia, one of the typical Japanese elements of the Gastropods, obtained from the basal part of the Miyazaki group. His contribution furnished a firm basis to MakiYama's scheme of the evolution of that genus with the illustration of an ancestral form.

In brief, valuable contributions have been made by several previous authors, especially to the field of biostratigraphy, but much has remained to be done in various aspects of stratigraphy and palaeontology. For instance, only the limited portion of the fauna was previously studied and consequently for grasping the general features of the whole fauna more study is necessary. More extensive and systematic field survey is needed for understanding the natural and reasonable relationships of the stratigraphic units. Palaeontological studies of the contained fossils could be extended along the lines of palaeoecology and evolution.

Under these situations I have been engaged in the stratigraphic and palaeontologic study of the Miyazaki group since 1948 at the suggestion of Professor Tatsuro Matsumoto. This study has the three-folded purposes; first to clarify the role of the Miyazaki group in the Caenozoic history in southwest Japan, especially in Kyushu Island, secondly to establish a biostratigraphic standard there for the basis of the intra- and interregional correlation, and thirdly to make evident the palaeoecological relation between the fossil assemblages and the enclosing rocks. I already described the results of the general stratigraphy 
and history of that group in two papers $(1952,1-40 ; 1958,643-653)$. I also issued several serial reports of the description of the mollusca (1955-1960) and a paper on the evolution of the mollusea $(1957,565-585 ; 636-647)$.

This report is a summary of the consecutive data for twelve years in realization of the main purposes in the palaeontological and biostratigraphical branches. It contains three principal results. The first is the result of the correlation of the faunal assemblages to other faunas, that concerns with biochronology and biostratigraphy. The second is about the composition of the fossil assemblages, the differentiation in the biofacies, and the relation among the biofacies, lithofacies, and the environmental factors. In other words, the second results are the palaeoecological remarks. The third concerns with the succession of the fossil assemblages, the lineages of the molluscan species and their speciation mechanics, that is about a problem of evolution.

The fossil material at my disposal comprises the collections of Professor T. MAtsumoto and the second year students in 1938 of the Department of Geology, Faculty of Science, Kyushu University and my own ones since 1948. All the described or listed specimens are registered and deposited in the type room of the Department of Geology, Kyushu University, Fukuoka.

\section{Acknowledgements}

This study is a part of the synthetic project, the study of the Caenozoic history in Kyushu Island and its adjacent, which was planned and started in 1948 under the direction of Professor Tatsuro Matsumoto. I wish to express my cordial thanks to him for his appropriate guidance and criticisms through which the project has been carried out safely and effectively. He also gave me thorough advices and suggestions at the typescript.

I am deeply indebted to Dr. Katura OYAMA of the Geological Survey of Japan, who led me into the taxonomic and ecologic field of the mollusca with kind advices and valuable criticisms. Drs. Tokubei KuRoDA of Kyoto University and Tadashige HABE of Kyushu University also gave me useful advices concerning the classification and ecology of the molluscan species and made me access to the publications and the specimens needed.

I am also deeply indebted to Professor Ryuzo ToRIYAMa of Kyushu University for his kind helps and encouragements in the course of the study and for his pertinent criticisms at the typescript. Mes. Kiyoshi TaKahashi, Yoshiro UyedA, and Ikuo OBATA of the same university, and especially Mr. Kôji FUJII of the Onoda Cement Co. Ltd. gave me valuable criticisms and advices on the course of the general study.

Professor Jiro MakiYama of Kyoto University, Teiichi Kobayashi and Fuyuji TAKaI of the University of Tokyo, and Nobuo IKEBE of Osaka City University made me access to the necessary specimens and the libraries.

Drs. Shoji IJIRI of the Association of the Geological Collaborators of Japan, Tamio KoTaKa of Tohoku University, Tetsuo HaNaI of the University of 
Tokyo, Mitoshi TokudA of Kyoto University, and Kazunori HuJita and Shigeru AokI of Tokyo University of Education gave me valuable criticisms and advices concerning the palaeoecology and the speciation problem.

Drs. Kazuyoshi IDA of the Geological Survey of Japan and Yusho OKaмото of Kyushu University, Ms. Mitsuo Suzuki and Tetsuji Hara gave me attentive advices and helps during the field survey. Miss Chizuko OKAMURA helped me in preparing the typescript.

I was deeply enlightened by Professor Ernst MAYER of Harvard University in general biological problems, especially in regards to the speciation of the animals and he made me access to the indispensable papers.

I wish to express here my hearty thanks to these persons without whose supports the project could not been executed.

This study was partly financed with a grant in aid by the Ministry of Education.

\section{A brief note on the lithostratigraphy of the Miyazaki group.}

The Miyazaki group is distributed in the Miyazaki district of southeast Kyushu from Takanabe Town on the north via Miyazaki City to Nichinan City on the south. The extent of the area is about $60 \mathrm{~km}$. from north to south and some $30 \mathrm{~km}$. from east to west.

Formerly the southern part of the Miyazaki group was called the Aburatsu series (M. SAKAWA, 1933, 1-48) or Aburatsu group (Y. OTukA, 1939, 481-519). The northern part was studied, irrespective of the southern part, by Y. OTUKA and named the Tsuma and Takanabe groups (OTUKA, 1930, 1048-1074). Some portion of the central part, surrounding Miyazaki City, was named the Miyazaki group by UCHIO (1947, 87-88). However the systematic field work for four years revealed the evidence that these stratigraphic units really belong to a single continuous sedimentary body (= MAKIYAMA's neptone) and are not separated by any unconformity or diastem. Thereupon I proposed that all these units should be united under a single group name, the Miyazaki group, which was named after the major city of the district (SHUTO, 1952, 1-40).

On northwest of the area occupied by the Miyazaki group the exceedingly thick Mesozoic strata, called the Shimanto supergroup, are extensively developed. On the southwest the Oligocene Nichinan and the Oligo- Miocene Sakatani groups are distributed in an intricate and mozaic pattern indicating the intensive disturbance by folding and faulting. These older rocks are overlain unconformably by the Miyazaki group.

The Miyazaki group consists of the very thick strata of various facies and displays rather simple structures with the gentle seaward dip. Along the strike-trend the strata form two shallow structural arcs, which face to east with their concave sides. The dip of the strata ranges between six to twenty degrees on the north arc and between fifteen to fourty degrees on the south one. These general arcuate distribution is moderately modified by the node and the fault structures. 
It contains three mega-facies* distributed from north to south: the Tsuma, the Miyazaki, and the Aoshima facies. The Tsuma facies is typified by the predomination of the monotonous unconsolidated thick siltstones. While the Aoshima facies is characterized by the very thick sequence of thin-cycled graded beds of hard sandstones and mudstones. The lithologic and diagenetic difference between the two facies is so great that they were considered to belong to the utterly different geological units of different ages. The Miyazaki facies is intergradational, being featured by the interfinger distribution of the Tsuma and the Aoshima facies, that is to say, it comprises mainly the large scale alternation of the relatively thick mudstones and the graded beds of a few ten-meters cycles.

The Miyazaki group, on the whole, also contains two major cycles of sedimentation in the vertical sequence. Consequently the stratigraphic details of the group are realized in this frame work. The recognized stratigraphic units are named and arranged in table $1 . * *$

Table 1. Stratigraphic subdivisions of the Miyazaki group.

\section{Miyazaki Group}

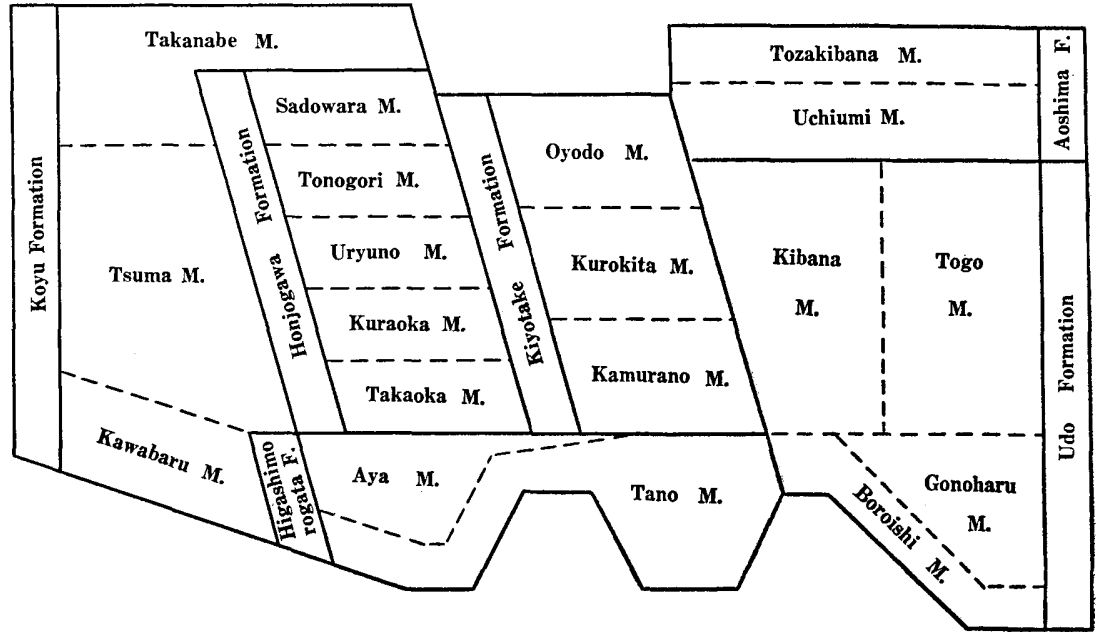

\section{I-Aoshima facies}

The strata of the Aoshima facies are divided into two formations, the lower, Udo and the upper, Aoshima formations, each of which represents a major cycle of sedimentation. The Udo formation is in turn subdivided into the Boroishi sandstone-conglomerate, the Gônoharu mudstone, and the very thick Kibana-Tôgô alternation members in ascending order. The Udo formation is much thicker than the Aoshima formation which contains the Uchiumi mudstone and the Tozakibana alternation members.

The Boroishi sandstone-conglomerate member unconformably covers the

* Moore, Raymond C., 1947, 1-34.

** See plate 1 and 2 . 
Oligocene Nichinan group. At the type section, from Mt. Boroishi to Kaichigô, it consists of the lower coarser and the upper finer parts. The lower part shows the ascending sequence of the basal conglomerate $(70 \mathrm{~m}$.), alternation of the conglomerates and the sandstones $(100 \mathrm{~m}$.), and pebble-bearing medium sandstone $(40 \mathrm{~m}$.), of which the second unit preserves a few molluscan remains and the last one contains numerous specimens of Operculina complanata japonica HANZAWA. The cobbles and the pebbles in these beds are well rounded, consisting of cherts, sandstones, slates, silicified grey shales, etc. The upper part, $190 \mathrm{~m}$., is generally predominated by the massive thick fine sandstones with the intercalated beds and lenses of pebble- or granule-conglomerates. It is rich in the calcareous nodules and sandpipes in its middle and the molluscan fossils in its upper portion.

The Boroishi member is a wedge-like unit which not only becomes thin southward but also decreases the amount of conglomerates in the same direction.

The Gônoharu mudstone member is also a wedge-like body thinning northwards and has the maximum thickness of about $400 \mathrm{~m}$. at the type section from Gônoharu to Kamigô in Kitagô mura, Minaminaka gun. The fine sandstone of the upper part of the Boroishi member grades upwards into the grey compact mudstone of Gônoharu member with the intercalation of thin alternation at the boundary. The main part of this member consists of the monotonous and poorly stratified mudstones and contains many arenaceous foraminifers throughout the beds but no available mollusks. The lower part is silty and contains a few molluscan remains.

The Kibana-Tôgô alternation members consist of the thin-cycled graded beds of hard sandstones and mudstones. In the northern part the member contains a few conglomerate beds and attains the thickness of about $1000 \mathrm{~m}$., which is the minimum figure of the member. Generally the sandstone layer is much thicker than the siltstone in each graded cycle there, but the relative thickness of a silty layer increases upwards and southwards, while the thickness of cycle does not change remarkably. The Kibana and the Tôgô members were respectively established with the northern type section along Kayeda river, where the sequence is predominated by sandstones, and with the southern type section from Masuyasu to Udo, where sandstones and mudstones are equally developed. The boundary of the two members is purely artificial on account of the gradational change of the lithological character.

In these two members several massive medium sandstones of three to eight meters in thickness are intercalated at several horizons, and they are generally very extensive in lateral continuation. For example the Bunsui sandstone, which is distributed along the main ridge of the coast range, is traced for about $15 \mathrm{~km}$. The pelecypods with thin tests rarely occur in the siltstones, while the foraminifers are rather common in them.

These members are overlain by the Uchiumi mudstone member of the Aoshima formation. Although the Uchiumi member is predominated by the mudstones, it is not at all so massive as the Gônoharu member, but is frequently stratified 
and intercalated by numerous sandstone layers at many horizons. The type section of this member is taken at Uchiumi, Miyazaki City. The upper part of this member is successively increased with the layers of sandstones and grades upwards into the Tozakibana alternation member without any abrupt change of the facies. Consequently the boundary between the two members is artificial.

The Tozakibana alternation member is typically developed at Tozakibana, the south extremity of Miyazaki City, where the member is composed of thin-cycled graded beds of sandstones and siltstones like as the Tôgô member and intercalated by a thin pumiceous tuff bed. The members of the Aoshima formation are also generally scarce of the molluscan remains.

\section{II-Tsuma facies}

In the Tsuma facies the stratigraphic sequence is divided on the lithological grounds into three parts which are named the Kawabaru conglomerate-sandstone, the Tsuma mudstone, and the Takanabe mudstone members in ascending order. They are grouped up into one formation, the Koyu formation.

The Kawabaru conglomerate-sandstone member covers unconformably the Mesozoic strata with its basal conglomerate. This member is unique by two characteristic features. The one is that the member has at various localities and horizons several beds and lenses of striped black mudstones (or muds) with grey streaks, rich in plant drifts. The other is that the member is frequently intercalated with the conglomerates and composed of the irregular alternation of cross-bedded sandstones, conglomerates, and mudstones, many of which are featured by the products of the penecomtemporaneous erosion and deformation such as the mudstone pebbles of irregular pan shape, truncation of some mudstones and sandstones, and intrastratal sliding. In other words, this member is predominated by the deltaic sediments, which especially developed in the area from Mino to Kamihokita with lignite seams. The upper most part of the member, however, is purely marine in origin and consists of the calcareous fine sandstones with the frequent intercalation of medium sandstones and mudstones, being rich in molluscan remains. It is very interesting that the axis of this fossil delta shifted northwards in the lapse of time. The thickness of the member is about $400 \mathrm{~m}$. in the type section from Kawabaru to Takajô in Kijô mura, where the sediments represent the later axis of the fossil delta and largely consist of pebble conglomerates and pebble-bearing sandstones. The maximum thickness is measured at Mino mura, the assumed earlier center of the delta.

The Tsuma mudstone member is extensively developed at Saito City (formerly Tsuma Town) and its suburbs and the type section is chosen along the left bank of the Hitotsuse river from Sugio to Yamasumi. It is almost wholly composed of grey, soft, massive, and friable mudstones with some intercalation of few sandy siltstones at the middle and a thin key tuff bed at the poorly stratified upper part. At the north area it overlies the alternation of the 
upper part of the Kawabaru member. While in the south area it succeeds the Aya mudstone member without any marked lithological change. The Tsuma member is rich in foraminifers, but impoverished in the molluscan fossils except for rare occurrence of such deep water forms as Limopsis, Acila, Nucula, Periploma, etc.

The Takanabe mudstone member overlies the Tsuma member and its type section is chosen from Taiheiji to Nihonmatsu via Kôonji in Takanabe Town. It consists dominantly of the stratified, soft, and pale grey siltstones with subordinately the associated sandy siltstones and fine sandstones, often exhibiting the irregular alternation. Comparing with the Tsuma member it differs apparently in a few features. First, frequent intercalation of the loose sand beds and soft sandstones of various thickness; second, the marked development of the stratification and even lamination in the siltstones except for a small part; third, common occurrence of the plant drifts in the fine beds especially in the foliated parts; fourth, the tendency of abrupt lateral change in lithofacies; and fifth, the pale coloration. Two tuffaceous horizons prolific in molluscan fossils are developed at the upper part east of Takanabe.

North of the Komaru river this member largely consists of the coarse siltstones, while south of the Hitotsuse river it contains the stratified thick sandstones and thin-cycled alternations, and the pebble- and shell-bearing contorted mudstones are developed in large scale, which indicate the submarine sliding during sedimentation.

\section{III-Miyazaki facies}

In the Miyazaki facies the strata are grouped into three formations, the Higashimorogata formation on the west, the Honjoggawa formation on the north, and the Kiyotake formation on the south. The first mentioned formation is subdivided into the Tano sandstone and the Aya mudstone members on the lithologic ground. The second is subdivided on the basis of the cyclic sedimentation into the Takaoka, Kuraoka, Uryuno, Tonogôri, and Sadowara sandstonemudstone members in ascending order. The third, on the same reason as the preceding one, is subdivided into the Kamurano, Kurokita, and ôyodo alternation members in ascending order.

The Tano sandstone member in the type section from west of Uyeharu to Hôkôbô in Tano Town begins with the thin basal conglomerate, which grades upwards into the large-cycled alternation of coarse and medium sandstones of bluish grey or greenish grey color with the pebble lenses and fossils such as Operculina complanata japonica HANZAWA at a few horizons and the imperfect molluscan remains at different horizons other than the Operculina horizons. These sandstones in turn grade upwards into rather monotonous and fossiliferous silty sandstones. This member is thickest at Tano and Aya "embayment" with the thickness of about 400 to $450 \mathrm{~m}$. and thin on Yusunoki "ridge", where the lithology is dominated by the frequent alternation of the coarse to medium sandstones with some boulder conglomerates, and the fossiliferous fine sandstone 
of upper part is only 40 to $60 \mathrm{~m}$. thick. In general the member is highly calcareous, monotonous, and rather massive in lithologic feature, but has occaionally the thinly alternating parts and the intraformational conglomerates as seen south of Aya Town. It changes gradually its lithology north of Aya, where the basal conglomerate and coarse sandstones with Operculina are very thin and overlain by the alternation of medium and fine sandstones with round pebbles, calcareous nodules, and molluscan remains. This alternating part towards its top gradually becomes finer but the monotonous silty sandstone, which features the southern area, is not developed.

The Aya mudstone member consists chiefly of massive compact mudstones, resembling the Gônoharu member in lithology and thickness, and represents the sediments at the time of the maximum subsidence of the transgressive phase. It is distributed in the area from Takaoka northwards to Sanzai via Aya and Yatsushiro with the type section along the right bank of the Ayanami river, south of Aya. In the type section it is exceedingly monotonous in lithology with the occasional intercalation of thin fine sandstones and attains about $500 \mathrm{~m}$. While it becomes abruptly thin and hard southwards through the replacement by the sandy alternation in its upper part. Northwards it is replaced at several horizons by irregular alternation of fine to silty sandstones and mudstones. It is also thining out dipwards rather abruptly. The lower boundary of the member is artificial in the type section and the southward adjacent part because of the perfectly gradational character of the vertical change in lithology. In the north area the conglomerate of the Kawabaru member is directly overlain by the Aya mudstone without any remarkable transition beds. In the intermediate area between the above mentioned parts the mudstone covers the irregular alternation with the comtemporaneous erosion-features. This member is rather scanty of the molluscan fossils like the Gônoharu member.

The Honjôgawa formation overlies the foregoing member and is distributed along the Honjô river (type section) and immediate south of it (Kuraoka and Takaoka). It is characterized by the alternating thick beds of mudstones and sandstones, the latter of which are the tongue bodies of narrow extent. Exhibiting the typical interfinger relation, they grade northwards to the mudstones of the Koyu formation and southwards to the thin-cycled alternation of the Kiyotake formation. The sandstone bodies of the lower part are generally massive and hard, on the contrary those of the upper part are rather loose, stratified, and friable. The higher the positions of the sandbeds, the more north their locations. Along the north-eastern edge of the sandstone bodies various abnormal features in sedimentation such as submarine sliding, submarine erosion, and other comtemporaneous deformation are observed at several localities. These features indicate that the Honjôgawa formation represents the sediments on the burried high, which acted periodically as positive element throughout medial and late Miocene time.

The molluscan remains are generally rare in the lower half of this formation except for a few fossil banks, but rather frequent in the upper half. 
T'he Kiyotake formation partly overlies the Aya member and in part directly covers the Tano member. It is distributed in the area between the Kayeda river on the south and the ôyodo river on the north with the type section along the Kiyotake river from west of Kamurano to Gunjibu. This formation is represented chiefly by the typical graded sequences of sandstones and mudstones separated by the intercalation of the compact sandy siltstones of about 5 to $30 \mathrm{~m}$. thick, and is subdivided into three members as follows.

The Kamurano alternation member, the lower member, is predominated by the stratified hard calcareous siltstones and featured by the subordinate constituents, the nodulous sandstones and conglomerates, at three horizons. The proportion of the siltstones to the sandstones gradually increases northwards in spite of the invariability in the thickness of each graded bed and it abruptly decreases southwards.

The Kurokita alternation member, the middle member, is composed of the alternations, which is predominated by the sandy materials in the lower half and by the mudstones in the upper half in the area north of the Kiyotake river. South of that river, however, the member is represented by the sand-prevalent alternations throughout the entire sequence. The thickness of the grading cycles in the alternation ranges from 10 to $50 \mathrm{~cm}$. in every area.

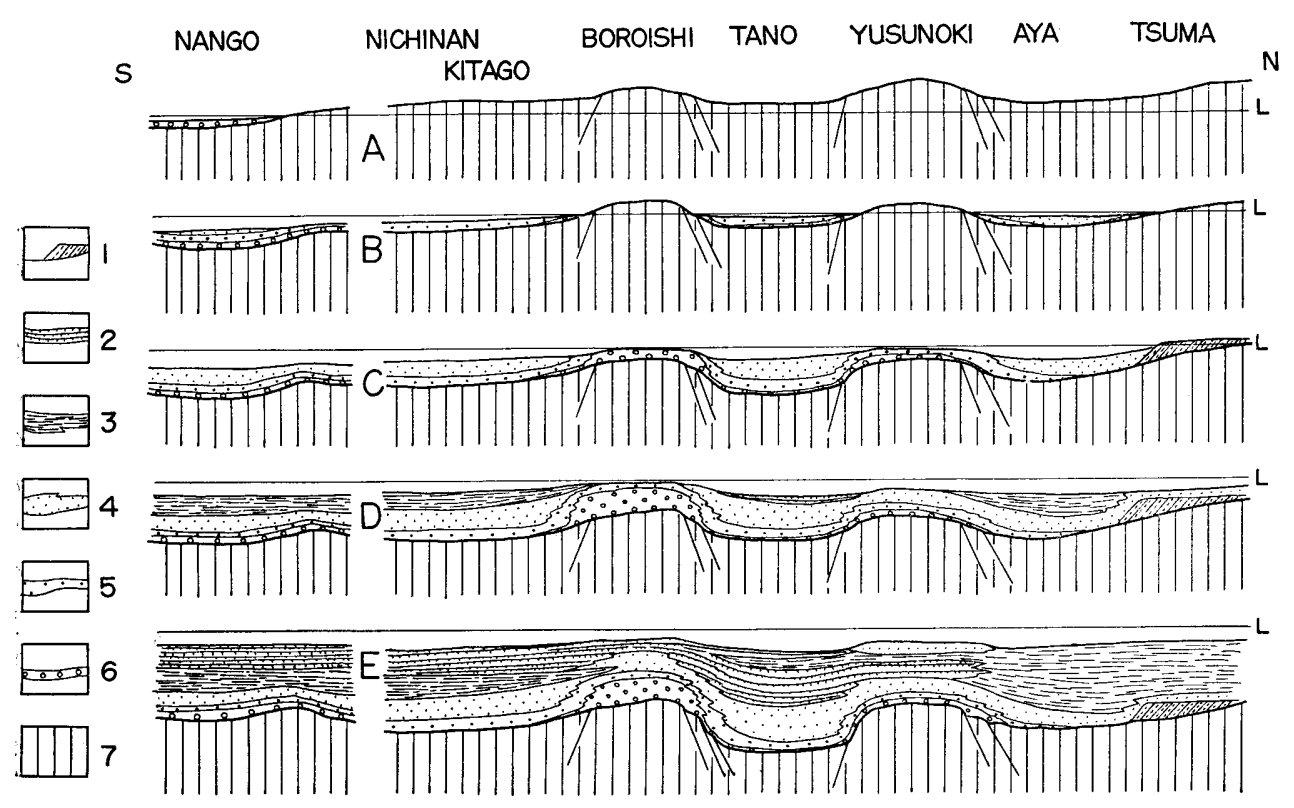

Text-fig. 2. Generalized cross section of the Miyazaki group showing the sedimentary frame work.

$\mathrm{A}-\mathrm{E}$ indicate the lower cycle of the sedimentation. Note the contrast between the positive and the negative elements.

1 .... deltaic sediment; $2 \ldots$. alternation of sandstones and mudstones;

$3 . .$. mudstone $; 4 \ldots$ fine sandstone; $5 \ldots$ medium and coarse sandstone ;

6 .... conglomerate ; 7 ... basement rocks. 
The ôyodo member, the upper member, consists of three cycles of sedimentation, each of which exhibits the lithologic change from mud-predominate to sand-predominate alternation from lower to upper. At the middle horizon of this member a thin tuff bed crops out, which is considered to be the continuation of the key tuff in the Tsuma member.

The abrupt lateral change in lithology between the Kiyotake formation and the sand-rich sequence of the Kibana member of the Udo formation can be explained by the assumption of a trough and a burried high.

The Kiyotake formation contains rare molluscan remains, but is rich in foraminifers and spines of echinoderms.

IV-Sedimentary history of the Miyazaki group

Judging from the relatively flat surface of the unconformity at the base of the Miyazaki group in comparison with the hardness of the basement rocks and from the limited distribution of the basal conglomerates of that group on the faulted ridges, the Miyazaki district must have suffered the intensive erosion before the invasion of the transgressive sea (late Helvetian)*, which deposited the Miyazaki group. While this monotonous flat topography and the simple coast line are modified by a few ridges. It is a matter of question whether these ridges represent the relict topography by differential erosion or the newly introduced tectonic highs. Whereas the important fault zones run along the landward continuation of these ridges and the ridges themselves are considered to have been the active elements during the sedimentary period. Accordingly I am inclined to consider that the ridges are of tectonic origin.

The transgressive sea which invaded the faulted area suddenly became deep to accumulate silty sediments even on the ridges (Tortonian), and then it became gradually shallower with minor fluctuations as the result of the approximate balance between the sinking of the basement and the deposition of the sediments (Sarmatian).

Examination of the fossil delta west of Tsuma reveals that the basal conglomerates in that area are rather meager and limited in distribution and the conglomerates of large scale are developed at higher horizons. This means the increase of the erosion on the backland owing to the upheaval or to the increase in the precipitation, of which the latter case is less possible, because the coarse sediments of this time are generally calcareous except for the deltaic part. In other words, the sinking on the basin was corresponding to the upheaval of the backland at least at the early stage of the Miyazaki group.

During the long period of equilibrium between sinking and sedimentation, the burried ridges especially the northern Yusunoki ridge developed a tendency to act as the positive elements and resulted to form on one hand the sand banks on the burried ridges and on the other hand the partially protected basin on the rear side of the ridge from the oceanic current. In other words the silty formations of the Tsuma area and the silt rich alternation of the

\footnotetext{
* The determination of ages indicated in ( ) is to be discussed in Chapter IV.
} 


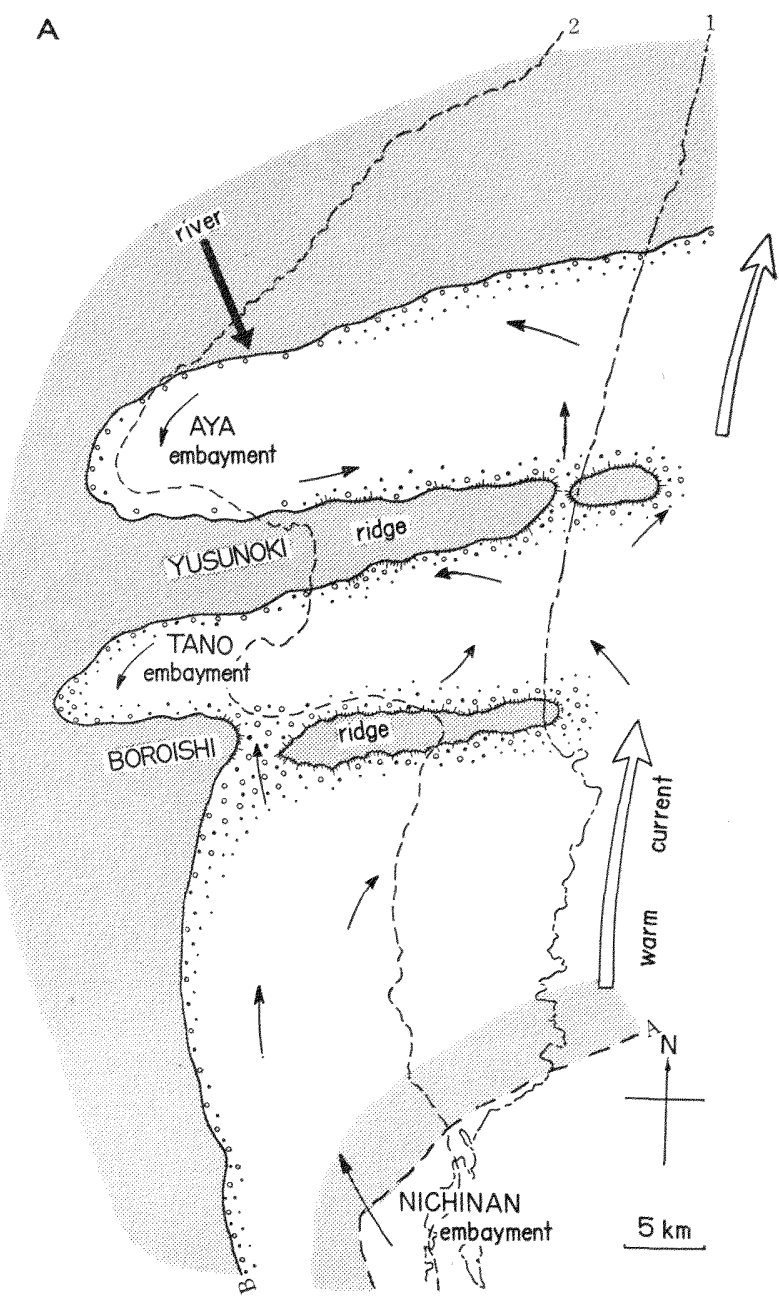

Text-fig. 3. Palaeogeographical map of the Miyazaki district at the early stage of the first transgression of the Mio-Pliocene cycles.

grey ground....land; dotted and circled area.... habitat of Operculina complanata japonica HANZA.WA; line A.... shore line at the beginning of the transgression; line B..., shore line at the time of the Operculina horizon; broken line $1 \ldots$ present shore line; broken line $2 \ldots$ pre sent boundary between the Miyazaki group and the basement.

Kiyotake area were respectively formed north of the Yusunoki and the Boroishi burried ridges. Reflecting the active tendency of the ridges the sediments on the sand banks occasionally slided down into the silty basin to form many irregular deposits.

After a long duration of the very gradual regressive phase a renewed transgression abruptly invaded this district with the accompaniment of the volcanism to form the finer sediments (Pontian) and then gradually withdrew 


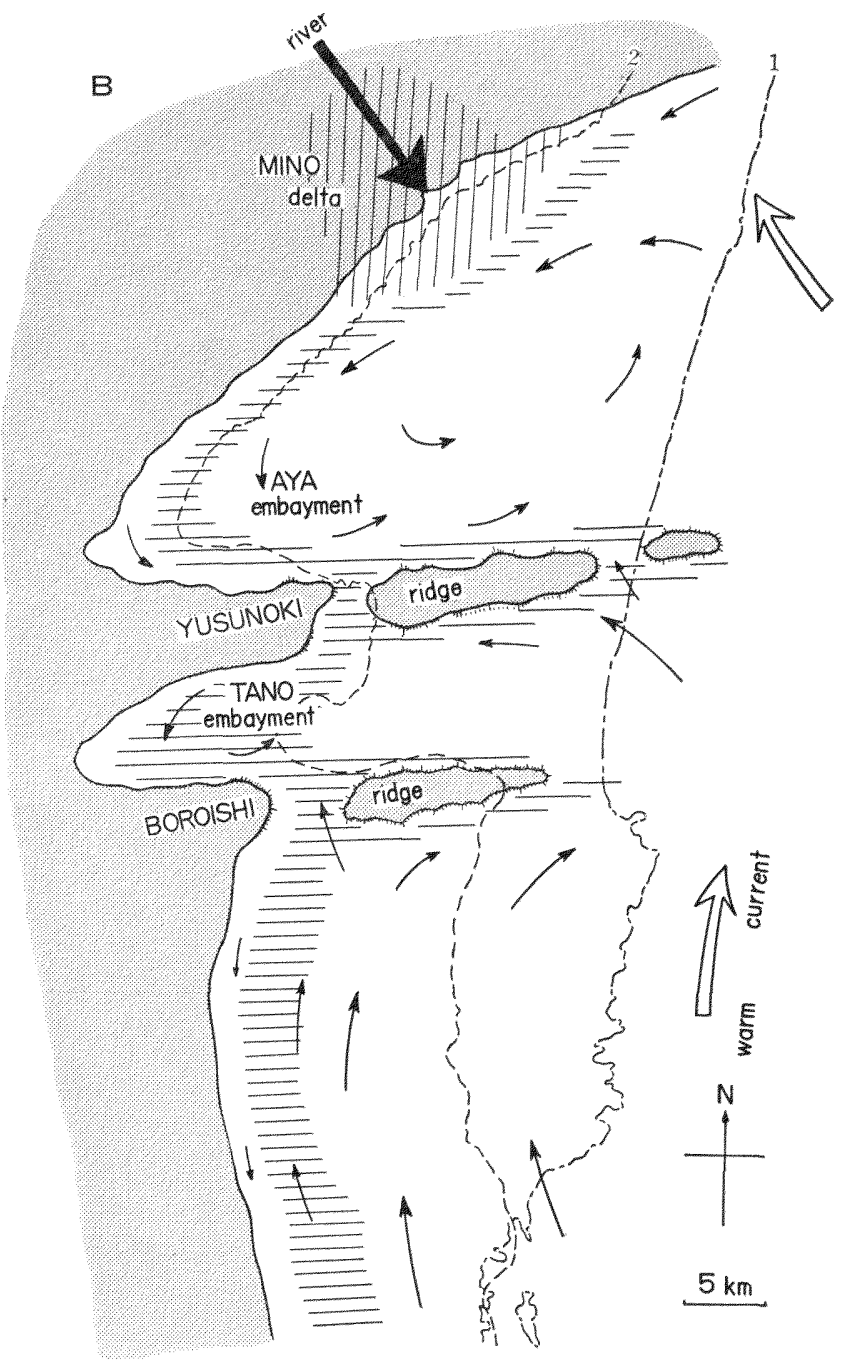

Text-fig. 4. Palaeogeographical map of the Miyazaki district at the time of the first horizon of the Miyazaki group.

grey ground... land; horizontally lined area... habitat of the neritic mollusca; broken line 1... present shore line; broken line 2...present boundary between the Miyazaki group and the basement.

from this area (Plaisancian).

\section{Complete list of the molluscan species from the Miyazaki group.}

The following is the complete list of the molluscan species obtained from the Miyazaki group except for the indeterminable ones. The geographical range and the bathymetric distribution of each species are shown in the second and third columns, respectively.

The figures in the second column indicate the north latitude along Pacific 


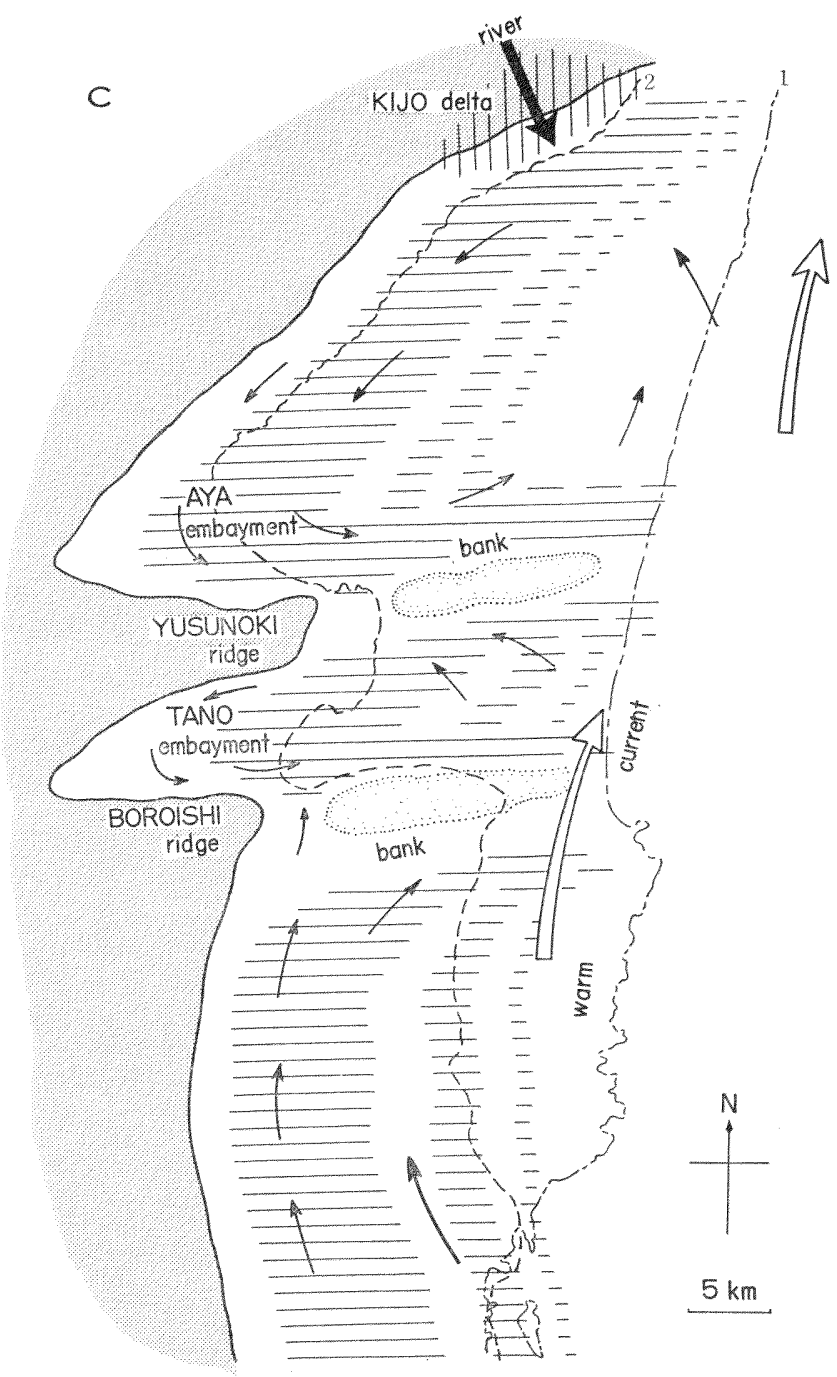

Text-fig. 5. Palaeogeographical map of the Miyazaki district at the time of the maximum subsidence resulting the accumulation of thick mudstones of the Aya and the Gônoharu member.

grey ground.... land; horizontally lined area....area of the mud accumulation; broken line $1 . .$. present shore line; broken line $2 . .$. present boundary between the Miyazaki group and the basement.

coast from Japan to Philippines.

In the third column $N_{0}, N_{1}, N_{2}, N_{3}, N_{4}$, and $B$ indicate the tidal (between high high tide and low low tide level), euneritic (between low low tide and 20$30 \mathrm{~m}$.), mesoneritic (between 20-30 and 50-60 m.), subneritic (between 50-60 and 100-120.), bathyneritic (between 100-120 and $200 \mathrm{~m}$.), and bathyal fascia (between 200-1000 m.), respectively (OYAMA, 1952, 27-35). 
Phylum Mollusca

Class Pelecypoda

Subclass Prionodesmacea

Order Taxodonta

Superfamily Nuculacea

Family Nuculidae

1 Nucula (Nucula) paulula (A. ADAMs)

Ennucula cfr. akitana OTUKA

Acila (Acila) divaricata (HINDS)

A. (A.) submirabilis MaKiYama

A. (Truncacila) minuta MakiYAma

Family Nuculanidae

6 Saccella confusa (HANLEY)

7 S. confusa kongiensis OTUKA

8 Nuculana (Nuculana) pennula (YoкоуамA)

9 Yoldia (Tepidoleda) naganumana (OTUKA) n. subsp.

10 Portlandia (Portlandella) japonica (ADAMS and ReEve)

11 Sarepta sp. efr. speciosa A. AdAMs

Superfamily Arcacea

Family Arcidae

12 Anadara (Scapharca) castellata (Yokoyama)

$\begin{array}{cc}33-39 & \mathrm{~N}_{1} \\ (35-39) & \left(\mathrm{N}_{1}-\mathrm{N}_{1}\right) \\ 30-35 & \mathrm{~N}_{1}-\mathrm{N}_{4} \\ - & (\mathrm{N}) \\ (33-35) & \left(\mathrm{N}_{2}\right) \\ & \\ 0-36 & \mathrm{~N}_{1}-\mathrm{B} \\ - & \left(\mathrm{N}_{1}-\mathrm{N}_{1}\right) \\ - & \left(\mathrm{N}_{1}-\mathrm{N}_{4}\right) \\ - & \left(\mathrm{N}_{4}-\mathrm{B}\right) \\ 33-34 & \mathrm{~N}_{1}-\mathrm{N}_{3} \\ 31-36 & \mathrm{~N}_{2}-\mathrm{N}_{4}\end{array}$

$-\quad\left(\mathrm{N}_{2}\right)$

$-\quad\left(\mathrm{N}_{0}-\mathrm{N}_{1}\right)$

$-\quad\left(\mathrm{N}_{0}-\mathrm{N}_{1}\right)$

$0-15 \quad \mathrm{~N}_{0}-\mathrm{N}_{1}$

A. (S.) daitokudoensis (MAKIYAMA)

A. (S.) rhombea (BoRN)

A. sp.

A. (Diluvarca) triceniosta (Nyst)

Pseudogrammatodon sp.

9 Barbatia (Barbatirus) cometa (REeve)

0 Acar plicata (DILlwYN)

21 Hawaiarca uwaensis (YokоYамA)

Family Cucullaeidae

22 Cucullaea granulosa (JoNAs)

23 C. pamotanensis MARTIN

Family Glycymeridae

24 Clycymeris rotunda (DUNKER)

25 G. totomiensis MAKIYAMA

26 G. formosana YoKoYAMA

27 Veletuceta nakamurai (MAкIYAMA)

28 V. reevei (MAYER)

Family Limopsidae

29 Limopsis obliqua A. AdAMs

30 Pectunculina oblonga (A. ADAMS)

31 Oblimopa forskalii (A. ADAMs)

Order Dysodonta

Superfamily Pteriacea

Family Mytilidae

32 Volsella nitida (REsve)

$31-35 \quad \mathrm{~N}_{3}$

Superfamily Pteriacea

Family Pteriidae

.33 Pteria sp.

Superfamily Pinnacea

25-35 $\overline{N_{3}}$

$-\quad\left(\mathrm{N}_{0}-\mathrm{N}_{\mathrm{i}}\right)$

14-26 $\mathrm{N}_{1}$

0-39 $\mathrm{N}_{0}-\mathrm{N}_{\text {. }}$

30-35 N- $\mathrm{N}_{\text {, }}$

0-35 $\mathrm{N}_{1}-\mathrm{N}_{\text {. }}$

$(0-10) \quad\left(N_{1}-N_{2}\right)$

31-42 N.-N,

- $\left(\mathrm{N}_{2}-\mathrm{N}_{3}\right)$

- $\left(\mathrm{N}_{1}-\mathrm{N}_{3}\right)$

$-\quad\left(\mathrm{N}_{1}-\mathrm{N}_{2}\right)$

0-31 $\mathrm{N}_{\mathrm{t}}-\mathrm{N}_{2}$

22-39 $\mathrm{N}_{3}-\mathrm{N}_{1}$

$34 \quad \mathrm{~N}_{z}-\mathrm{N}_{1}$

23-39 $\mathrm{N}_{-2}-\mathrm{N}_{4}$ 
Family Pinnidae

34 Atrina (Servatrina) pectinata (LINNÉ)

$0-36 \quad \mathrm{~N}_{1}-\mathrm{N}_{2}$

35 A. (Atrina) (REEVE)

0-33 $\quad \mathrm{N}_{3}-\mathrm{N}_{4}$

Superfamily Pectinacea

Family Pectinidae

Subfamily Pectininae

36 Gloripallium satowi (YoKоYAMA)

- $\quad\left(\mathrm{N}_{1}-\mathrm{N}_{2}\right)$

37 G. miurensis (YoKoYaMA)

38 G. aurantiacus (SOWERBY)

39 Mimachlamys miniacea (LAMARCK)

40 Cryptopecten vesiculosus (DUNKER)

- $\quad\left(\mathrm{N}_{1}-\mathrm{N}_{2}\right)$

20-26 $\quad \mathrm{N}_{1}-\mathrm{N}_{2}$

0-32 $\mathrm{N}_{1}-\mathrm{N}_{2}$

41 Patinopecten taiwanus NomurA

42 Palliolum (Delectopecten) peckhami (GABB)

0-35 $\quad \mathrm{N}_{-}-\mathrm{N}_{3}$

3 P. (D.) macrocheiricola HABE

- $\quad\left(\mathrm{N}_{1}-\mathrm{N}_{2}\right)$

$-\quad\left(\mathrm{N}_{3}-\mathrm{N}_{4}\right)$

$0-35 \quad\left(\mathrm{~N}_{3}-\mathrm{B}\right)$

Subfamily Amussiinae

44 Amusium n. sp.

45 Amussiopecten praesignis (YokOYAMA)

46 A. iitomiensis (ОTUKA) *

47 Parvamussium (Parvamussium) kyushuense SHuto

Family Limidae

48 Lima (Acesta) goliath (SMITH)

$$
\begin{array}{ll}
- & \left(\mathrm{N}_{2}-\mathrm{N}_{4}\right) \\
- & \left(\mathrm{N}_{1}-\mathrm{N}_{2}\right) \\
- & \left(\mathrm{N}_{1}-\mathrm{N}_{2}\right) \\
- & \left(\mathrm{N}_{3}-\mathrm{B}\right)
\end{array}
$$

$10-35 \quad \mathrm{~N}_{1}-\mathrm{B}$

Superfamily Anomiacea

Family Anomiidae

49 Anomia lischkei Dautzenberg and Fischer

$23-42$

$N_{10}-N_{i}$

50 A. sp.

Superfamily Ostreacea

Family Ostreidae

51 Pycnodonta musashiana (Yokoyama)

52 Ostrea (Crassostrea) gigas (THuMBERG)

26-36 $\quad \mathrm{N}_{10}-\mathrm{N}_{\text {: }}$

$23-43 \quad \mathrm{~N}_{0}-\mathrm{N}_{1}$
53 Nodostrea sp.

Subclass Teleodesmacea

Order Heterodonta

Superfamily Astartacea

Family Crassatellidae

54 Crassatellites (Crassatellites) takanabensis SHuTo

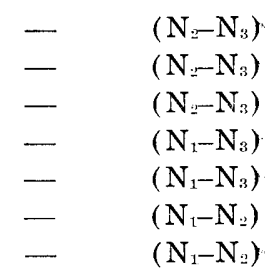

55 C. (C.) tenuiliratus tenuiliratus SHUTo

56 C. (C.) tenuiliratus triangularis SHUTo

57 C. (C.) tsumaensis Shuto

58 C. (C.) tosaensis Nomura

59 C. (Crassatina) oblongatus uchidanus (YoKoyama)

60 Scambra (Eucrassatella) sp.

Superfamily Carditacea

Family Carditidae

61 Cardita sp.

62 Venecricardia (Megacardita) panda (YoKoYAMA)

$\begin{array}{cc}- & - \\ - & \left(\mathrm{N}_{1}-\mathrm{N}_{2}\right) \\ - & \left(\mathrm{N}_{1}-\mathrm{N}_{2}\right) \\ - & \left(\mathrm{N}_{1}-\mathrm{N}_{3}\right) \\ 31-35 & \mathrm{~N}_{1}-\mathrm{N}_{3} \\ - & \left(\mathrm{N}_{1}-\mathrm{N}_{2}\right)\end{array}$
$63 V .(M$.$) oyamai SHuTo$

$64 V .(M$.$) megacostata Shuto$

$65 V .(M$.$) ferruginosa (A. AdAMs and ReEve)$

$66 V .(M$.$) granulicostata Nomura$

Superfamily Lucinacea
$\left(\mathrm{N}_{1}-\mathrm{N}_{4}\right)$

* Amussiopecten iitomiensis (OTUKA) $=$ A. hyugaensis SHUTo. 
Family Ungulinidae

67 Joannisiella cumingii (HANELY)

$\begin{array}{cc}33-35 & \mathrm{~N}_{1}-\mathrm{N}_{2} \\ 33-35 & \mathrm{~N}_{1}-\left(\mathrm{N}_{2}\right) \\ 33-45 & \mathrm{~N}_{1}-\mathrm{N}_{3} \\ & \\ 33-35 & \mathrm{~N}_{-}-\mathrm{N}_{1} \\ - & \left(\mathrm{N}_{2}-\mathrm{N}_{4}\right) \\ (36-56) & \mathrm{N}_{+} \\ 31-41 & \mathrm{~N}_{3} \\ - & \mathrm{N}_{2} \\ - & - \\ 23-41 & \cdot \\ - & \mathrm{N}_{0}-\mathrm{N}_{4} \\ 32-36 & \left(\mathrm{~N}_{1}\right) \\ & \mathrm{N}_{1}-\mathrm{N}_{2}\end{array}$

68 J. lunaris (YoKоYaMA)

69 Felaniella usta (Gould)

Family Thyasiridae

70 Thyasira (Thyasira) tokunagai KURODA and HABE

71 T. n. sp.

72 T. (Conchocele) nipponica $\mathrm{Y}_{\mathrm{ABE}}$ and Nomura

Family Lucinidae

73 Lucinoma annulata (REEVE)

74 L. acutilineata (CONRAD)

75 L. sp.

76 Pillucina pisidium (DUNKER)

77 P. (Sydlorina) efr. yokoyamai OTUKA

78 Bellucina civica (YoкоYамA)

Superfamily Ericinacea

Family Montacutidae

79 Mysella n. sp.

Superfamily Cardiacea Family Cardiidae

80 Vasticardium ogurai (ОTUKA)

$81 V$. hyugaense SHUTо

82 Vepricardium kyushuense Sнuto

83 Trachycardium shiobarense (YoKoYAMA)

84 Dinocardium braunsi (ToKUNAGA)

85 Clinocardium shinjiense (Үокоуама)

86 C. subdecussatum SHUTO

87 Laevicardium pigmaea SHUTo

88 Fulvia mutica (REEve)

89 Nemocardium (Keenaea) samarangae (MAKIYAMA)

Superfamily Veneracea Family Veneridae Subfamily Venerinae

90 Venus (Ventricoloidea) foveolata miyazakiensis (SнUто) Subfamily Pitarinae

91 Pitar (Pitarina) dohrni (ROEMER)

92 P. ( $P$.) pellucida (LAMARCK)

93 P. (P.) sulfurea (PILSBRY)

94 P. (P.) semeliformis SнUто

95 P. (Agriopoma) sp.

96 Callista (Callista) chinensis (HoLten)

97 C. (C.) roscida (Gould) Subfamily Dosiniinae

98 Dosinia (Phacosoma) troscheli LISCHKE

99 D. (P.) nomurai ОтUкA

100 D. (Dosinorbis) bilunulata (GRAY)

101 D. (Bonartemis) suketoensis (OTUKA)

102 Kaneharaia kaneharai (Yокоунма)

Subfamily Clementiinae

103 Clementia (Clcmentia) papyracea (Gray)

Subfamily Tapetinae

$1^{04}$ Paphia (Paphia) takanabensis Shuto
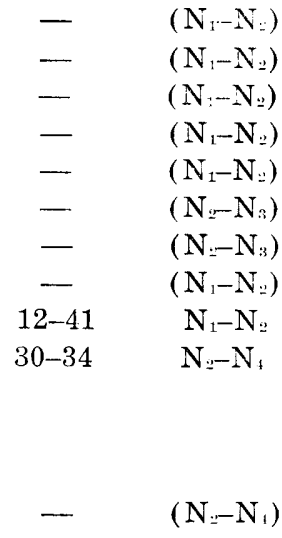

$0-10 \quad \mathrm{~N}_{1}-\mathrm{N}_{2}$

14-29 $\mathrm{N}_{1}-\mathrm{N}_{3}$

23-35 $\quad \mathrm{N}_{1}-\mathrm{N}_{3}$

- $\left(\mathrm{N}_{1}-\mathrm{N}_{3}\right)$

- $\quad\left(\mathrm{N}_{1}-\mathrm{N}_{3}\right)$

23-39 $\quad \mathrm{N}_{1}-\mathrm{N}_{\text {. }}$

26-33 $\mathrm{N}_{\mathrm{t}}-\mathrm{N}$ :

30-39 $\quad \mathrm{N}_{1}-\mathrm{N}$.

- $\quad\left(\mathrm{N}_{1}-\mathrm{N}_{\mathrm{i}}\right)$

31-35 $\mathrm{N}_{1}-\mathrm{N}_{2}$

- $\left(\mathrm{N}_{1}-\mathrm{N}_{2}\right)$

$-\left(\mathrm{N}_{1}-\mathrm{N}_{2}\right)$

$0-29 \quad \mathbf{N}_{1}$

$-\quad\left(N_{i}-N_{*}\right)$ 


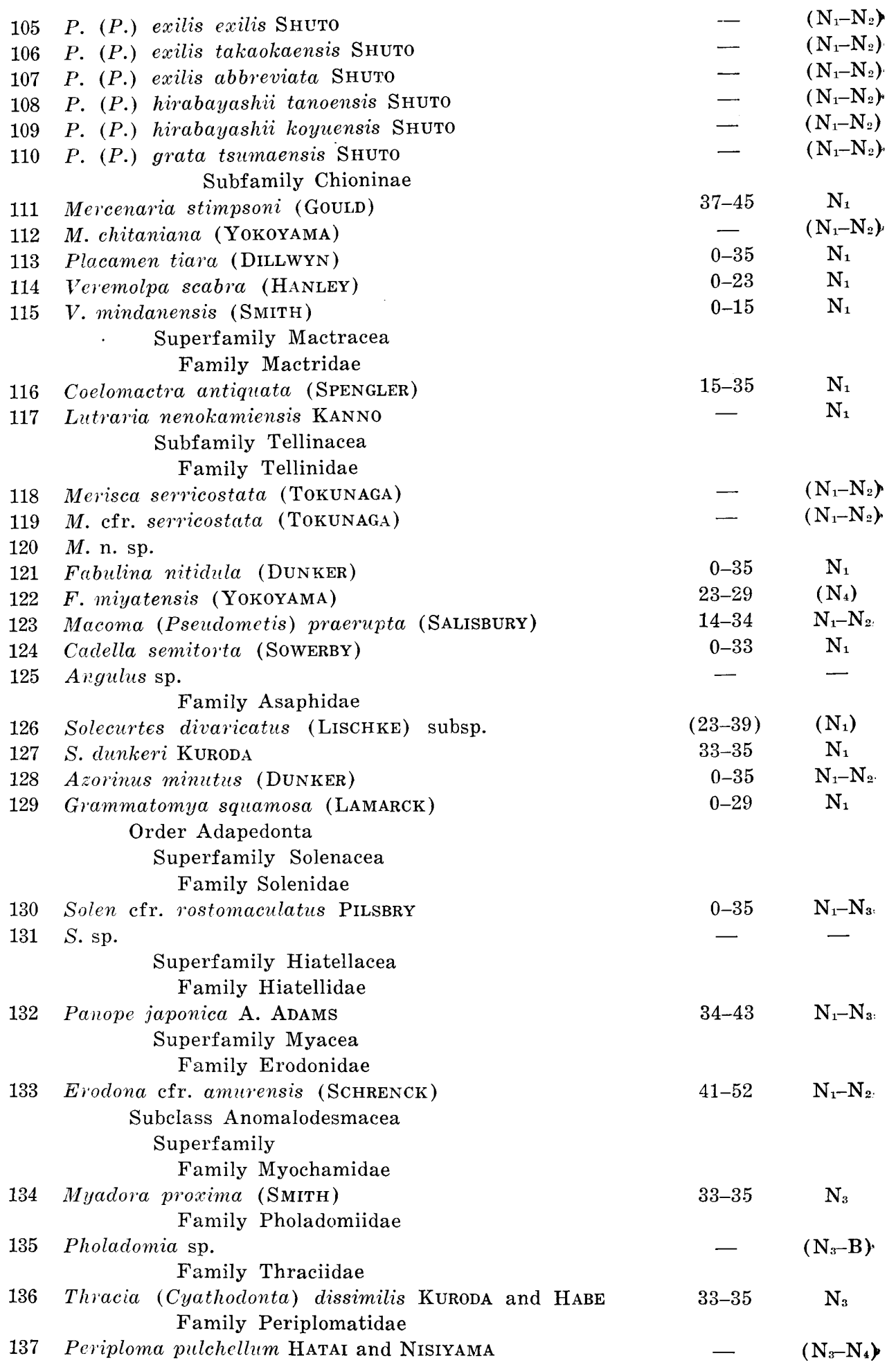


P. besshoensis (YoKoYAMA) n. subsp.

$$
\begin{array}{cc}
- & \left(\mathrm{N}_{3}-\mathrm{N}_{4}\right) \\
32-35 & \mathrm{~N}_{2}-\mathrm{B} \\
- & \left(\mathrm{N}_{2}-\mathrm{B}\right) \\
- & \left(\mathrm{N}_{2}-\mathrm{B}\right) \\
- & \left(\mathrm{N}_{2}-\mathrm{B}\right) \\
- & \left(\mathrm{N}_{2}-\mathrm{B}\right) \\
- & \left(\mathrm{N}_{2}-\mathrm{B}\right) \\
- & \left(\mathrm{N}_{2}-\mathrm{B}\right) \\
- & \left(\mathrm{N}_{2}-\mathrm{B}\right)
\end{array}
$$

Superfamily Poromyacea

Family Cuspidariidae

Cuspidaria (Cuspidaria) hindsiana (A. ADAMs)

C. (C.) n. sp. a

C. (C.) n. sp. b

C. (C.) n. sp. c

C. (Myonella) n. sp

C. (M.) sp.

C. (Cardiomya) n. sp. a

C. (C.) n. sp. b

Class Scaphopoda

147 Dentalium yokoyamai MaKIYAMA

148 D. (Paradentalium) octangulatum Donovan

149 D. (P.) hexagonum Gould

$(29-34) \quad\left(\mathrm{N}_{4}\right)$

$0-42 \quad \mathrm{~N}_{1}-\mathrm{N}_{2}$

0-42 $\quad \mathrm{N}_{1}$

150 D. (Antalis) totomiensis MaKiYama

$-\quad\left(\mathrm{N}_{2}-\mathrm{N}_{3}\right)$

151 Siphonodentalium (Siphonodentalium) nipponicum MAKIYAMA

152 S. (Pulserum) ozavai (YokoYAMA)

Class Gastropoda

Subclass Prosobranhia

Order Archaeogastropoda

Superfamily Trochacea

Family Trochidae

Subfamily Margaritinae

153 Lischkeia alwinae (LIScHKE)

154 L. n. sp.

155 Turcicula argenteonitens convexiusculla (YoKoYAMA)

156 Turcica sp.

Subfamily Calliostomatinae

157

C. $\mathrm{sp}$.

Subfamily Monodontinae

Calliostoma (Otukaia) kounjianum (Yoкоуама)

C. (Tristichotrochus) shinagawaense cipangoanum (YoKOYAMA)

Tegula (Chlorostoma) yokoyamai Nomura and HATAI

Subfamily Umboniinae

Suchium (Suchium) suchiense subsuchiense MAKIYAMA

S. (S.) obsoletum arenarium MAKIYAMA

S. (S.) koyuense SHUTo

S. (S.) n. sp.

S. (Protorotella) hyugaensis SHUTo

S. (P.) maxima Shuto

Ethalia (Ethaliella) ? sp.

Subfamily Trochinae

Trochus sp. a

T. sp. b

Family Skeneidae

170 Teinostoma pusillum (C. B. AdAMs)

171 Starkeyna sobrina (A. ADAMs)

Superfamily Rissoacea

Family Tornidae

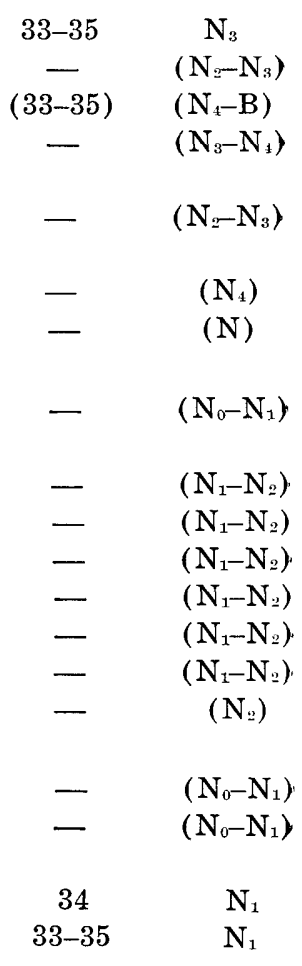


173 Turritella (Turritella) perterebra Yокоуама

$\begin{array}{ll}- & \left(\mathrm{N}_{1}-\mathrm{N}_{2}\right) \\ - & \left(\mathrm{N}_{2}-\mathrm{N}_{3}\right) \\ - & \left(\mathrm{N}_{2}-\mathrm{N}_{3}\right)\end{array}$

175 T. (H.) n. sp. b

Subfamily Orectospirinae

176

Orectospira excelsa (YoKoYAMA)

Family Architectonidae

177 Philippia cinglata (KIENER)

178 Architectonia (Stellaxis) n sp.

Family Cerithiidae

Subfamily Cerithiinae

179 Bittium binodulosum Yокоуама

180 B. sp.

Superfamily Scalacea

Family Scalidae

181

Scala (Boreoscala) nagamiensis OTUKA

Superfamily Eulimacea

Family Eulimidae

182. Melanella (Melanella) sp.

M. (Balcis) musta (YokoYama)

Family Pyramidellidae

184 Pyramidella (Tibera) sp. a and b

185 Turbonilla (Tropaeas) sp.

186 T. (Turbonilla) sp. a and b

187 Odostomia (Odostomia) hilgendorfi (ClEssin)

188 O. (O.) sp. a and b

189 Syrnola sp.

190 Kleinella (Leucotina) sp.

191 Menestho (Oscilla) sp.

Superfamily Calyptraeacea

Family Calyptraeidae

192 Calyptraea yokoyamai KURODA

193 C. sp.

194 Crepidula cfr. isimotoi ОтUкA

195 C. sp.

Superfamily Strombacea

Family Xenophoridae

196 Xenophora (Tugurium) exuta (REEve)

$197 X .(T$.$) dunkeri MarTiN$

\section{Superfamily Naticacea}

Family Naticidae

198 Polinices (Mammilaria) sagamiensis (PILSBRY)

199 Neverita (Glossaulax) reiniana (DUNKER) n. subsp.

200 N. (G.) didyma (RoEDING)

201 N. (G.) didyma bicolor (PHILIPPI)

202 Mammila simiae (Deshayes)

203 Uberella yokoyamai KURODA and HABE

204 U. rufa (BORN)

$205 U$. n. sp.
$(33-35) \quad\left(\mathrm{N}_{2}-\mathrm{N}_{3}\right)$

$0-35 \quad \mathrm{~N}_{-}-\mathrm{N}_{3}$

$-\quad\left(\mathrm{N}_{2}-\mathrm{N}_{3}\right)$

$\left(\mathrm{N}_{1}\right)$

$\left(\mathrm{N}_{1}\right)$

- $\quad\left(\mathrm{N}_{1}\right)$

$-\quad\left(\mathrm{N}_{1}\right)$

$\left(\mathrm{N}_{1}\right)$

- $\quad\left(\mathrm{N}_{1}-\mathrm{N}_{1}\right)$

- $\left(\mathrm{N}_{\mathrm{i}}-\mathrm{N}_{+}\right)$

$34-42 \quad \mathrm{~N}_{1}$

- $\quad\left(\mathrm{N}_{1}\right)$

- $\quad\left(\mathrm{N}_{1}\right)$

$-\quad\left(N_{1}\right)$

$-\quad\left(\mathrm{N}_{1}\right)$

31-35 N

- $\quad\left(\mathrm{N}_{1}\right)$

- $\quad\left(\mathrm{N}_{1}\right)$

$-\quad\left(\mathrm{N}_{1}\right)$

0-35 $\quad \mathrm{N}_{2}-\mathrm{N}_{3}$

$(0-10) \quad\left(\mathrm{N}_{-}-\mathrm{N}_{3}\right)$

$\begin{array}{cc}31-35 & \mathrm{~N}_{1}-\mathrm{N}_{:} \\ (31-35) & \left(\mathrm{N}_{1}-\mathrm{N}_{3}\right) \\ 0-42 & \mathrm{~N}_{0}-\mathrm{N}_{3} \\ 0-29 & \mathrm{~N}_{1}-\mathrm{N}_{3} \\ 0-35 & \mathrm{~N}_{1} \\ - & \left(\mathrm{N}_{1}\right) \\ 0-15 & \mathrm{~N}_{1} \\ - & \left(\mathrm{N}_{1}\right)\end{array}$


206 Tectonatica janthostomoides KURODA and HABE

207 T. andoi Nomura

Superfamily Cypraeacea

Family Amphiperatidae

208 Volva sp.

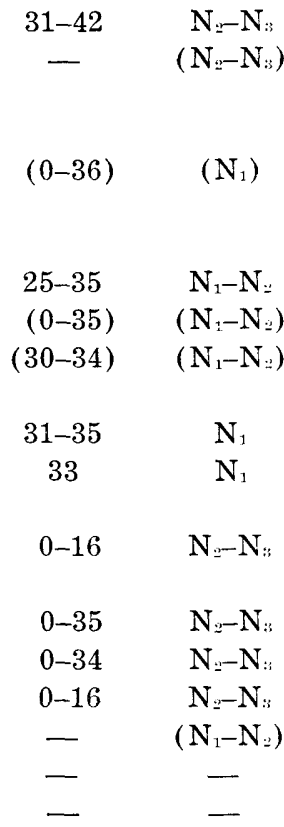

$(0-34) \quad\left(N_{1}-N_{2}\right)$

Order Neogastropoda

Superfamily Muricacea

Family Muricidae

Subfamily Rapaninae

222 Rapana aff. bulbosa (REEve)

Subfamily Columbariinae

223 Columbarium pagodum (LEsson)

Subfamily Muricinae

224 Poirieria azami KURodA

225 Chicoreus pliciferoides KURODA

226 Murex (Murex) cfr. verbecki MARTIN

227 M. (M.) cfr. djarianensis MARTIN

228 M. (M.) n. sp.

Subfamily Drupinae

229 Ocenebra adunka (SowerBy)

Subfamily Typhiinae

230 Siphonochelus (Siphonochelus) japonicus (A. ADAMs)

Superfamily Buccinacea

Family Pyrenidae

231 Pyrene bicinctella (Yokoyama)

232 P. lischkei (SMITH)

Family Buccinidae

233 Babylonia elata (YoKoyama)

234 B. areolata (LINK)

235 Plicifusus sp.

236 Ancistrolepis (Clinopegma) unicus (PILSBRY)

237 Siphonalia declivis declivis Yoroyama

$0-35 \quad \mathrm{~N}$

32-35 $\quad \mathrm{N}_{3}-\mathrm{N}$

10-35 $\mathrm{N}_{:-}-\mathrm{N}$

29-35 $\quad \mathrm{N}_{:-\mathrm{N}_{4}}$

$-\left(\mathrm{N}_{1}-\mathrm{N}_{2}\right)$

- $\left(\mathrm{N}_{1}-\mathrm{N}_{2}\right)$

$-\left(\mathrm{N}_{1}-\mathrm{N}_{2}\right)$

25-39 $\mathrm{N}_{2}-\mathrm{N}_{\text {, }}$

33-33 $\mathrm{N}$

(33-35) $\quad\left(\mathrm{N}_{1}-\mathrm{N}_{2}\right)$

24-39 $\mathrm{N}_{1}-\mathrm{N}$.

$\begin{array}{cc}- & \left(\mathrm{N}_{1}\right) \\ 0-24 & \mathrm{~N}_{1} \\ - & - \\ 38-42 & \mathrm{~N}_{:-\mathrm{N}_{4}} \\ - & \left(\mathrm{N}_{1}-\mathrm{N}_{: 2}\right) \\ - & \left(\mathrm{N}_{1}-\mathrm{N}_{:}\right)\end{array}$


239 S. declivis n. sp. a

240 S. declivis n. sp. b

241 S. dainitiensis MAKIYAMA

242 S. mikado Melville

243 S. praedeclivis ITOIGAWA

244 S. spadicea fuscolineata (PEASE)

245 S. cfr. tonohamaensis totomiensis MAKIYAMA

246 S. cfr. ikebei TsudA

247 Nassaria magnifica LischKe

248 N. magnifica LISCHKE n. subsp.

249 N. lischkei MAKIYAMA

250 Kelletia sp.

251

252 Nassarius (Zeuxis) caelatus dainitiensis MAKIYAMA

253 N. (Zeuxis) caelatus (A. AdAMs) n. subsp.

254 N. (Tritia) festivus (PowYs) n. subsp.
N. (Hinia) liratus (DUNKER) Family Fasciolariidae

Granulifusus dualis (YoKoYAMA)

G. koyuanus SHUTo

G. matsumotoi SHUTO

Fusinus simplex SMITH

F. perplexus (A. AdAMs)

Fasciolaria sp.

Family Galeodidae

Hemifusus ternatanus (GMELIN)

H. cfr. tuba (GMELIN)

H. sp.

Superfamily Volutacea

Family Olividae

Oliva (Oliva) mustellina LAMARCH

O. (O.) ispidula (LINNE)

Olivella (Olivella) spretoides YoKoxama

O. (O.) baetica Carpenter

O. (O.) consobrina (LISCHKE)

O. (O.) fulgurata (A. ADAMS and REEVE)

Ancilla (Baryspira) albocallosa okawai (YokoYama)

A. (B.) oyamai SHUTo

A. (Turrancilla) otukai SHUTo

A. (T.) miserula (YokoYAMA)

Family Mitridae

Mitra (Nebularia) hanleyana (DUNKER)

Fuscomitra sp.

Family Voltidae

Fulgoraria (Fulgoraria) cancellata KURodA and HABE

$F$. (F.) daviesi Fulton

F. (Psephaea) kamakuraensis OtukA

$F$. (P.) kosibensis OTUKA

F. (P.) sp.

Lyria mizuhonica mizuhonica MAKIYAMA

L. mizuhonica koyuana (YoKoYAMA)

Uromitar nakamurai ugariensis MAKIYAMA

$\begin{array}{cc}- & \left(\mathrm{N}_{1}-\mathrm{N}_{2}\right) \\ - & \left(\mathrm{N}_{1}-\mathrm{N}_{2}\right) \\ - & \left(\mathrm{N}_{1}-\mathrm{N}_{2}\right) \\ 31-35 & \mathrm{~N}_{3} \\ - & \left(\mathrm{N}_{1}-\mathrm{N}_{2}\right) \\ 31-35 & \mathrm{~N}_{2}-\mathrm{N}_{4} \\ - & \left(\mathrm{N}_{1}-\mathrm{N}_{3}\right) \\ - & \left(\mathrm{N}_{1}-\mathrm{N}_{3}\right) \\ 33-35 & \mathrm{~N}_{3} \\ (33-35) & \left(\mathrm{N}_{3}\right) \\ 33-35 & \mathrm{~N}_{3} \\ - & \left(\mathrm{N}_{2}\right) \\ - & \left(\mathrm{N}_{1}\right) \\ & \\ - & \left(\mathrm{N}_{1}\right) \\ - & \left(\mathrm{N}_{1}\right) \\ - & \left(\mathrm{N}_{1}\right) \\ 29-33 & \mathrm{~N}_{1}\end{array}$

$\begin{array}{cl}- & \left(\mathrm{N}_{2}-\mathrm{N}_{4}\right) \\ - & \left(\mathrm{N}_{2}-\mathrm{N}_{4}\right) \\ - & \left(\mathrm{N}_{2}-\mathrm{N}_{4}\right) \\ 29-34 & \mathrm{~N}_{3}-\mathrm{N}_{4} \\ 31-42 & \mathrm{~N}_{1}-\mathrm{N}_{2}\end{array}$

0-35 $\quad \mathrm{N}_{1}-\mathrm{N}_{3}$

$9 \sim 34 \quad \mathrm{~N}_{1}-\mathrm{N}_{3}$

$\begin{array}{cc}11-35 & \mathrm{~N}_{1} \\ 0-33 & \mathrm{~N}_{1} \\ - & \left(\mathrm{N}_{3}-\mathrm{N}_{4}\right) \\ - & \left(\mathrm{N}_{1}-\mathrm{N}_{2}\right) \\ 29-35 & \mathrm{~N}_{1}-\mathrm{N}_{2} \\ 25-39 & \mathrm{~N}_{3} \\ - & \left(\mathrm{N}_{2}-\mathrm{N}_{3}\right) \\ - & \left(\mathrm{N}_{2}-\mathrm{N}_{3}\right) \\ - & \left(\mathrm{N}_{4}-\mathrm{B}\right) \\ - & \left(\mathrm{N}_{3}-\mathrm{N}_{4}\right)\end{array}$

25-35 $\quad \mathrm{N}_{1}$

$\begin{array}{cc}33 & \mathrm{~N}_{3}-\mathrm{B} \\ 33-34 & \mathrm{~N}_{4}-\mathrm{B} \\ - & \left(\mathrm{N}_{3}-\mathrm{N}_{4}\right) \\ - & \left(\mathrm{N}_{3}-\mathrm{N}_{4}\right) \\ - & - \\ - & \left(\mathrm{N}_{1}-\mathrm{N}_{2}\right) \\ - & \left(\mathrm{N}_{1}-\mathrm{N}_{2}\right) \\ - & \left(\mathrm{N}_{2}\right)\end{array}$


Family Cancellariidae

285 Cancellaria (Cancellaria) reeveana laticostata LoEBECKE

286 C. (Merica) pristina (YoKoYAMA)

287 Sydaphera spengleriana (DESHAYES)

288 S. spengleriana (DESHAYES) subsp.

289 Trigonostoma kurodai MAKIYAMA

Family Marginellidae

290 Marginella novemprovincialis (YokoYAMA)

291 M. flaccida (YokoYama)

$$
\begin{array}{cc}
31-35 & \mathrm{~N}_{2}-\mathrm{N}_{4} \\
- & \left(\mathrm{N}_{2}\right) \\
0-39 & \mathrm{~N}_{2} \\
- & \left(\mathrm{N}_{2}\right) \\
- & \left(\mathrm{N}_{1}-\mathrm{N}_{2}\right) \\
- & \left(\mathrm{N}_{1}\right) \\
- & \left(\mathrm{N}_{1}\right)
\end{array}
$$

Superfamily Conacea

Family Turridae

Subfamily Turrinae

292 Turris (Polystira) kurodai Makiyama

293 T. (Turris) cfr. ugariensis MAKIYAMA

294 Ptychosyrinx n. sp.

295 Gemmula granosa (HeLBLING)

296 G. granosa (Helbling) n. subsp.

297 G. n. sp.

298 Micantapex n. sp.

299 Bathytoma striatotuberculata (YoKoYAMA)

300 B. luedorfi (LISCHKE)

301 B. n. sp. a

302 B. n. sp. b

303 Optoturis n. sp.

Subfamily Turriculinae

304 Turricula (Surcula) sobrina (Yokoyama)

305 T. (S.) n. sp.

306 T. (Orthosurcula) australis (RoISSY)

307 T. (O.) pervirgo (YoKoYaMA)

308 Fusituricula n. sp.

Subfamily Cochlespirinae

309 Ancistrosyrinx osawanoensis TsuDA

310 Cosmasyrinx n. sp.

311 Spirotropis subdeclivis (Yokoyama)

312 S. subdeclivis (YoKоYAMA) n. subsp.

313 Leucosyrinx creanica (A. AdAMS and REEVE)

314 Parasyrinx sp.

Subfamily Clavatulinae

315 Clavatula (Paradrillia) djocdjocartae serrana Fischen

316 C. (P.) astuta (YoKoYaMA)

317 C. (P.) dainichiensis (YoKoYAMA)

318 C. (P.) n. sp.

Subfamily Conorbiinae

319 Surculites siogamensis NomURA

320 S. yokoyamai OTUKA

Subfamily Clavinae
Cymatosyrinx rinsuikawaensis Nomura

C. osawanoensis TSUDA

Inquisitor (Inquisitor) jeffreysii (SMITH)

Pseudoinquisitor pseudoprincipalis (YoKoYAMA)

$P$. totomiensis ugariensis MAKIYAMA

P. hyugana (YoKoYAMA)

Comitas sp.

Tomopleura subdifficilis MakiYama

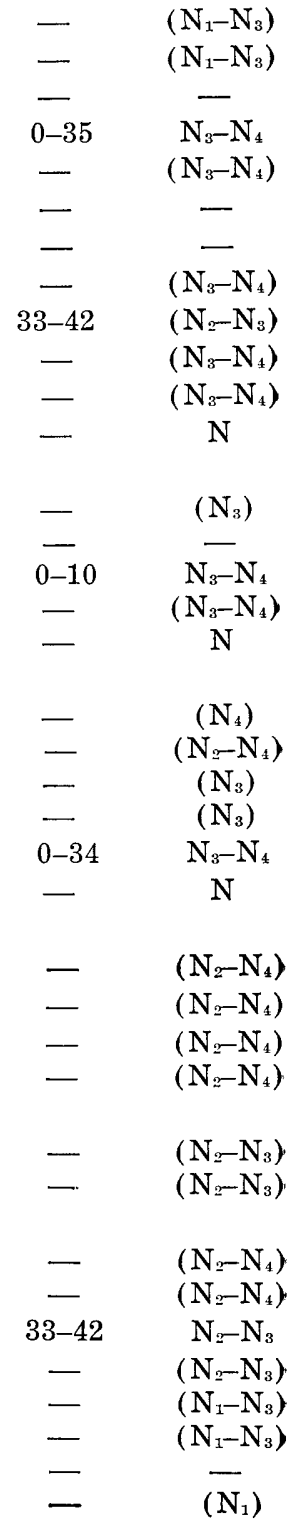


Borsonia n. sp. a

B. n. sp. b

Subfamily Mangeliinae

332 Etrema hayasakai NomurA

333 Lienardia n. sp.

334 Bellaspira (Lyromangilia) semicarinata (PILsBRY)

335 Propebella cf. yokoyamai ONoYAMA

336 Antiguraleus sp.

337 Filodrillia n. sp.

338 Thatchelia gradata (YoKoYAMA) Family Conidae

$$
\begin{array}{cc}
- & \left(\mathrm{N}_{1}\right) \\
- & - \\
- & - \\
- & \left(\mathrm{N}_{1}\right) \\
26-35 & \left(\mathrm{~N}_{1}\right) \\
- & \mathrm{N}_{2}-\mathrm{N}_{: 3} \\
- & - \\
- & - \\
- & \left(\mathrm{N}_{1}\right) \\
28-33 & \mathrm{~N}_{: 3} \\
- & \left(\mathrm{N}_{:}-\mathrm{N}_{:}\right) \\
0-29 & \mathrm{~N}_{1}-\mathrm{N}_{2} \\
0-35 & \mathrm{~N}_{1} \\
(33-37) & \left(\mathrm{N}_{1}\right) \\
- & \left(\mathrm{N}_{1}\right) \\
- & \left(\mathrm{N}_{1}\right) \\
27-31 & \mathrm{~N}_{1} \\
- & \left(\mathrm{N}_{1}\right) \\
- & \left(\mathrm{N}_{1}\right) \\
&
\end{array}
$$

339 Asprella (Asprella) comatosa (PILSBRY)

340 A. (Endemoconus) toyamaensis (TSUDA)

341 Floracons sp. Family Terebridae

342 Myurella (Myurella) naumanni (YoKoYAMA)

343 M. (Pervicacia) recticostata (YoKoYAMA)

$344 M$. (P.) sp.

346 S. (P.) orthocostulata (NomURA)

348 Microtrypetus sp.

349 Terebra sp.

Subclass Opithobranchia

Order Tectibranchia

Superfamily Cephalaspidea

Family Pupidae

350 Pupa strigosa (GouLd)

25-38 $\mathrm{N}_{1}$

351 Acteon kirai HABE

Family Ringiculidae

352 Ringicula (Ringiculina) doliaris (GoULD)

353 R. (R.) teramachii $\mathrm{HABE}$

Family Retusidae

354 Acteocina (Decolifer) oyamai HABE

31-34 N

31-42 $\mathrm{N}_{1}-\mathrm{N}_{2}$

$33 \quad \mathrm{~N}_{1}$

31-35 $\mathrm{N}:-\mathrm{N}:$

355 Retusa sp.

Family Philinidae

356 Philine scalpta A. Adams
$-\left(\mathrm{N}_{1}\right)$

34-35 $\mathrm{N}_{1}-\mathrm{N}_{\text {. }}$

\section{Palaeoecology of the molluscan faunas}

I-Descriptions of the localities and the fossil assemblages

The Miyazaki group contains a great number of the mega-fossil localities to be sorted into several horizons as listed below. In addition there are some unlisted localities, where only few and imperfect fossils have been found. In the following list the lithology, mode of preservation and occurrence of the fossils, specific names, and other remarks, if any, are given for each locality. The symbols prefixed at the species names, $\bullet, \odot$, or $\bigcirc$ indicate the categories: dominant, characteristic, or accompany species, respectively. The species without 
these symbols are of rare occurrence.

(1)-Kaburake (NH-3721), Nangô machi, Minami-Naka gun (N. 31 $31^{\prime} 13^{\prime \prime}$, E. $\left.131^{\circ} 23^{\prime} 17^{\prime \prime}\right)$

Fossil bed: Calcareous silty sandstone; quartz grains dominant; lower part of the Boroishi member.

Preservation: Moderately favourable, almost all the specimens identified are conjoined; shell materials remarkably recrystallized.

Occurrence: Sporadic, rare.

Andara (Scapharca) valentula (YoкоYамa), A. (S.) daitokudoensis MakiYama and other indeterminable specimens.

(2)-Setare (NH-3704), Nangô machi, Minami-Naka gun (N. 31 $31^{\prime} 45^{\prime \prime}$, E. $131^{\circ} 23^{\prime} 24^{\prime \prime}$ )

Fossil bed: Calcareous silty sandstone of grey color; lower part of the Boroishi member.

Preservation: Perfect.

Occurrence: Sporadic, rare.

Anadara (Scanpharca) rhombea BoRN, Dosinia sp.

(3)-Kiyama (NH-1274), Aburatsu, Nichnan City ('N. $31^{\circ} 35^{\prime} 24^{\prime \prime}$, E. $131^{\circ} 24^{\prime} 1^{\prime \prime}$ )

Fossil bed: Quartz rich fine sandstone, rarely stratified; somewhat weathered; upper part of the Boroishi member.

Preservation: Imperfect; shell matter greatly removed; greater parts of the specimens are conjoined.

Occurrence: Dence but scattered.

Nuculana sp., Portlandia (Portlandella) japonica (AdAms and ReEve), Anadara (Scapharca) valentula (Yokoyama), A. (S.) daitokudoensis MakiYama, O Amussiopecten iitomiensis (OTUKA), $\odot$ Vasticardium ogurai (OTUKA), - Paphia (Paphia) exilis takaokaensis SнUto, Epitonium (Boreoscala) nagamiensis OTUKA. (4) -Uchinota (MI-7590), rail-road side, northern extremity of Nichinan City (N. $31^{\circ} 38^{\prime} 22^{\prime \prime}$, E. $131^{\circ} 23^{\prime} 55^{\prime \prime}$ )

Fossil bed: Calcareous very fine sandstone, scarcely stratified, grey in color; upper part of the Boroishi member.

Preservation: Almost perfect, but conjoined valves rare.

Occurrence: Sporadic in muddy and rather massive part and densely crowded in a few thin sandstone lenses.

- Paphia (Paphia) exilis takaokaensis SuUto, Dosinia (Phacosoma) sp., Cuspidaria (Cuspidaria) n. sp.

(5)-Inohaye (MI-7449), $1800 \mathrm{~m}$. north of Inohaye along the Kamigo river, Kitagô mura, Minami Naka gun (N. $31^{\circ} 44^{\prime} 10^{\prime \prime}$, E. $131^{\circ} 22^{\prime} 26^{\prime \prime}$ )

Fossil bed: Grey silty sandstone, slightly calcareous, stratified, rather hard; upper part of the Boroishi member.

Preservation: Moderately favourable.

Occurrence: Dense but sporadic, pelecypod valves almost separated.

Amussiopecten iitomiensis (OTUKA), Venericardia (Megacardita) megacostata Shuto, Clementia (Clementia) papyracea Gray, Paphia (Paphia) exilis taka- 
okaensis SHUTO, Solecurtes divaricatus LISCHKE, Eudolium sp. c.

(6) -Kaichigô-a (MI-2694), $2000 \mathrm{~m}$. south of Maruno, Kibana, Miyazaki City (N. $31^{\circ} 47^{\prime} 50^{\prime \prime}$, E. $131^{\circ} 24^{\prime} 13^{\prime \prime}$ )

Fossil bed: Grey, hard, somewhat calcareous fine sandstone with silty matrix, scarcely stratified; the upper part of the Boroishi member.

Preservation: Fairly well, but the shell matter was partially removed.

Dentition of pelecypods and other detailed features are rarely observable.

Occurrence: Sporadic; conjoined valves are rare.

OAmussiopecten iitomiensis (OTUKA), $\odot$ Paphia (Paphia) exilis takaokaensis Sнuto, Solecurtes divaricatus LischKe, Architectonica (Stellaxis) n. sp., Balanus sp.

(7) - Kaichigô-b (MI-2705), $3200 \mathrm{~m}$. southwest of Maruno, Kibana, Miyazaki City (N. $31^{\circ} 47^{\prime} 16^{\prime \prime}$, E. $131^{\circ} 23^{\prime} 33^{\prime \prime}$ )

Fossil bed: Round pebble bearing grey medium sandstone, rarely stratified.

Middle part of the Boroishi member.

Preservation: Imperfect; greater parts of the specimens are fragmental.

Occurrence: Sporadic.

Amussiopecten iitomiensis (OTUKA), Paphia (Paphia) exilis takaokaensis SHuto, Siphonalia praedeclivis ITOIGAWA

(8) -Kaichigô-c (MI-2699), 2500 m. south of Maruno, Kibana, Miyazaki City (N. $31^{\circ} 47^{\prime} 12^{\prime \prime}$, E. $131^{\circ} 24^{\prime} 2^{\prime \prime}$ )

Fossil bed: Grey silty sandstone, almost massive; middle part of the Boroishi member.

Preservation: Favourable but shell substance is partly replaced.

Occurrence: Spradic, rare.

Paphia (Paphia) exilis takaokaensis SHuto, Merisca n. sp.

(9)-Northwest cliff of Mt. Boroishi (MI-2824), Kiyotake machi, Miyazaki gun (N. $31^{\circ} 48^{\prime} 19^{\prime \prime}$, E. $131^{\circ} 23^{\prime} 33^{\prime \prime}$ )

Fossil bed: Bluff medium sandstone of the lower part of the Boroishi member. Moderately stratified, calcareous and well-sorted.

Preservation: Favourable; conjoined valves not rare.

Occurrence: Rare, sporadic.

Amussiopecten iitomiensis (OTUKA), Trachicardium shiobarense (YoKoYAMA) and many other indeterminated specimens.

(10)-Takeuchi (MI-1077), right bank of Kayeda river, $600 \mathrm{~m}$. south of Takeuchi,

Kibana, Miyazaki City (N. $31^{\circ} 17^{\prime} 59^{\prime \prime}$, E. $131^{\circ} 24^{\prime} 30^{\prime \prime}$ )

Fossil bed: Grey, hard, massive silty sandstone of the upper part of the Boroishi member.

Preservation: Imperfect; shells are not worn, but the shell substance was largely removed through diagenetic process.

Occurrence: Sporadic and conjoined valves common.

Portlandia (Portlandella) japonica (ADAMs and REEve), Palliolum (Delectopecten) peckhami (GABR), $\odot$ Thyasira (Conchocele) nipponica YABE and NomurA, - Lucinoma acutilineata (CONRAD), Ancistrolepis (Clinopegma) unicus (PILSBRY). 
(11)-Maruno (MI-2466), $500 \mathrm{~m}$. southeast of Maruno, Kibana, Miyazaki City (N. $31^{\circ} 48^{\prime} 46^{\prime \prime}$, E. $\left.131^{\circ} 24^{\prime} 15^{\prime \prime}\right)$.

Fossil bed: Very hard, grey calcareous, scarcely stratified silty sandstone of the upper part of the Boroishi member.

Preservation: Rather favourable with preserved minutue ornamentation; shells are not worn, but the shell substance was partially removed.

Occurrence: Sporadic, conjoined valve rare.

Venericardia (Megacardita) ferruginosa (ADAMs and REEve), Vepricardium kyushuense Shuto, Dosinia sp. indet., Lutraria nenokamiensis Kanno, $\odot$ Merisca n. sp., Azorinus minutus (DUNKER).

(12) - Kagamisu pass (MI-2495), road side cutting $500 \mathrm{~m}$. north east of Kagamisu, Preservation: Favourable; minute ornamentation is observed in many Fossil bed: Grey, hard, somewhat calcareous silty fine sandstone intercalated in the fine sandstones of the upper part of the Boroishi member.

Preservation: Favourable; minute ornamentation is observed in many specimens.

Occurrence: Abundant but sporadic; conjoined valves common.

OSaccella confusa kongiensis OTUKA, Joannisiella cunimgii (HANLEY), Laevicardium pigmaea Sнuто, $\bigcirc$ Vepricardium kyushuense SHuto, Ventricoloidea foveolata miyazakiensis (SHuto), O Paphia (Paphia) exilis takaokaensis SHUto, Clementia (Clementia) papyracea GRAY, Solecurtes divaricatus LISCHKE, Solen sp., Turritella (Haustator) n. sp., Architectonica (Stellaxis) n. sp.

(13) $-400 \mathrm{~m}$. northeast of Kagamisu pass (MI-2610), Kiyotake machi, Miyazalki gun (N. $31^{\circ} 49^{\prime} 40^{\prime \prime}$, E. $131^{\circ} 23^{\prime} 48^{\prime \prime}$ ).

Fossil bed: Grey, hard, somewhat calcareous silty fine sandstone intercalated in the irregular alternation of medium to silty sandstones of the upper part of the Boroishi member.

Preservation: Fairly suitable; conjoined valves are not rare.

Occurrence: Abundant but sporadic; large shells are rare.

Acila (Acila) submirabilis MakiYama, $\odot$ Saccella confusa kongiensis Otuka, Yoldia (Tepidoleda) naganumana (OTUkA) n. subsp., Palliolum (Delectopecten) peckhami (GABB), Amussiopecten iitomiensis (OTUKA), Joannisiella cumingii (HANley), O Laevicardium pigmaea Shuto, Pitar (Agriopoma) sp., O Paphia (Paphia) exilis takaeokaensis SHuto, Clementia (Clementia) papyracea Gray. (14)-Matsugano (MI-2593), foot of the hill, $300 \mathrm{~m}$. west of Matsugano, Kiyotake machi, Miyazaki gun (N. $31^{\circ} 49^{\prime} 49^{\prime \prime}$, E. $131^{\circ} 22^{\prime} 59^{\prime \prime}$ ).

Fossil bed: Grey, hard, scarcely stratified silty fine sandstone with coarse sand mixture of the upper part of the Boroishi member.

Preservation: Imperfect; determinable specimens rare; shell matter is hardly preserved.

Occurrence: Rare, sporadic.

$\odot$ Paphia (Paphia) exilis takaokaensis Shuto, Solecurtes divaricatus LIScHKe, Eudolium sp. c, Balanus sp.

(15)-Obira (MI-1028), 700 m. southeast of Obira, road side, Tano machi, 
Miyazaki gun (N. $31^{\circ} 50^{\prime} 42^{\prime \prime}$, E. $\left.131^{\circ} 20^{\prime} 25^{\prime \prime}\right)$.

Fossil bed: Grey, hard, rarely stratified silty fine sandstone with coarse sand mixture of the upper part of the Tano member; small ferruginous nodules and plant drifts are common.

Preservation: Detailed characteristics are hardly observed on account of the partial removement of the shell substance.

Occurrence: Common, sporadic.

Saccella confusa kongiensis OTUKA, $\bigcirc$ Amussiopecten iitomiensis (ОTUKA), Joannisiella cumingii (HANLEY), $\odot$ Paphia (Paphia) exilis takaokaensis SHUTo, Clementia (Clementia) papyracea GRAY, Xenophora (Tugurium) sp., Carcinoplax antiqua (RISTORI).

(16)-Hôkôbô (MI-3121), $800 \mathrm{~m}$. southeast of Hokobo, river side, Tano machi, Miyazaki gun (N. $31^{\circ} 51^{\prime} 39^{\prime \prime}$, E. $131^{\circ} 19^{\prime} 16^{\prime \prime}$ ).

Fossil bed: Thick, monotonous, grey, somewhat calcareous sandy siltstone; scarcely stratified; upper part of the Tano member.

Preservation: Almost perfect; perfect specimens of crabs common.

Occurrence: Abundant but sporadic; greater parts of the pelecypod specimens are conjoined.

Saccella confusa kongiensis OTUKA, Amussiopecten iitomiensis (OTUKA), Joannisiella cumingii (HANley), Paphia (Paphia) exilis exilis SHUTo, $\bigcirc P$. (P.) exilis takaokaensis ShUTo, P. (P.) hirabayashii tanoensis ShUTo, $\odot$ Clementia (Clementia) papyracea GRAY, Fusinus sp., O Schizaster miyazakiensis MoRISHITA. Carcinoplax antiqua (RISTORI).

(17) - Kariyabaru (MI-903), river side, north of Kariyabaru, Tano machi, Miyazaki gun (N. $31^{\circ} 50^{\prime} 52^{\prime \prime}$, E. $\left.131^{\circ} 17^{\prime} 38^{\prime \prime}\right)$.

Fossil bed: Imperfectly sorted mixture of silt and sand; hard, grey, somewhat calcareous, and irregularly stratified.

Preservation: Not always perfect; shell material was partially removed in some specimens, though the shell surface was not worn before deposition.

Occurrence: Abundant but sporadic.

Amussiopecten iitomiensis (OTUKA), Joannisiella cumingii (HANLEY), O Paphia (Paphia) exilis takaokaensis SHUTO, OP. (P.) hirabayashii tanoensis SHUTo, Clementia (Clementia) papyracea GRAY, Lutraria nenokamiensis KANNo, Calliostoma sp., Xenophora (Tugurium) dunkeri MARTin, Phalium sp., Eudolium sp. $\mathrm{b}$ and c, Siphonalia praedeclivis ItoIGAWA, Murex (Murex), n. sp., Turris cfr. ugariensis MAKIYAMA, Turricula sp., Floraconus sp., Schizaster miyazakiensis MORISHITA, Carcinoplax antiqua (RISTORI).

(18) - Haigano (MI-1434), small cliff of the tributary of the Tano river, $700 \mathrm{~m}$. south of Haigano, Tano machi, Miyazaki gun (N. $31^{\circ} 51^{\prime} 16^{\prime \prime}$, E. $131^{\circ} 20^{\prime} 3^{\prime \prime}$ ). Fossil bed: Grey, somewhat brittle, scarcely stratified silty sandstone with calcareous nodules; upper part of the Tano member.

Preservation: Perfect; even minute characteristics are preserved.

Occurrence: Dense but sporadic, not crowded; conjoined valves are not rare. Ammusiopecten iitomiensis (OTUKA), O Joannisiella cumingii (HANLEY), Paphia 
(Paphia) exilis exilis Shuto, $\odot P .(P$.$) exilis takaokaensis Shuto, P$. (P.) exilis abbreviata SHUTo, O Clementia (Clementia) papyracea GRAY, Ventricoloidea foveolata miyazakiensis (SHUTo), Solecurtes divaricatus LISchKE, Cuspidaria (Myonella) n. sp., Euspira sp., Eudolium sp. c, Siphonalia praedeclivis ItoIGaWa, Schizaster miyazakiensis MORISHITA, Carcinoplax antiqua (RISTORI).

(19) - Uyeharu-a (MI-913), river side $700 \mathrm{~m}$. northwest of Uyeharu, Tano machi, Miyazaki gun (N. $31^{\circ} 50^{\prime} 23^{\prime \prime}$, E. $131^{\circ} 17^{\prime} 2^{\prime \prime}$ ).

Fossil bed: Irregularly stratified, grey, hard, calcareous silty fine sandstone with coarse sand mixture of the upper middle part of the Tano member.

Preservation: Shell matter was partly removed and the detailed feature of the shells is not generally observed.

Occurrence: Common, sporadic; broken shells not rare.

Amussiopecten iitomiensis (OTUKA), O Paphia (Paphia) exilis takaokaensis Shuto, Clementia (Clementia) papyracea Gray, Hemifusus cfr. tuba (GMELIN). (20) -Uyeharu-b (MI-3293), rail road side, $1100 \mathrm{~m}$. northwest of Uyeharu, Tano machi, Miyazaki gun (N. $31^{\circ} 50^{\prime} 44^{\prime \prime}$, E. $\left.131^{\circ} 16^{\prime} 28^{\prime \prime}\right)$.

Fossil bed: Almost massive, hard, greenish grey medium sandstone with silty matrix. Middle part of the Tano member.

Preservation: Shell matter was greatly removed and the detailed feature of the shell is not observable.

Occurrence: Rare and sporadic.

$\odot$ Laevicardium pigmaea SHUTo, $\odot$ Solen cfr. rostomaculatus PILsBRy.

(21) - Horiguchi (MI-820 to 822 ), road side $300 \mathrm{~m}$. south of Horiguchi, Tano machi, Miyazaki gun (N. $31^{\circ} 52^{\prime} 1^{\prime \prime}$, E. $131^{\circ} 18^{\prime} 49^{\prime \prime}$ ).

Fossil bed: Grey to greenish grey, hard, medium to fine sandstone with silty patches and lenses. Upper part of the Tano member.

Preservation: Imperfect; shell matter was partly removed.

Detailed characteristics are in many cases not ooserved even on the moulds because of the coarseness of the matrix.

Occurrence: Common to rare; sporadic.

Amussiopecten iitomiensis (OTUKA), Venericardia (Megacardita) megacostata Shuto, Joannisiella cumingii (HANLEY), Clinocardium shinjiense (YoKoYAMA), OVepricardium kyushuense SHUTo, $\odot$ Paphia (Paphia) exilis takaokaensis Shuto, $P$. (P.) hirabayashii tanoensis SHuto, Clementia (Clementia) papyracea GRAY, Lutraria nenokamiensis KANNo, Solecurtes divaricatus LISCHKE, Panope japonica A. ADAms, Cantharus sp.

(22)-Uchinohaye (MI-826), road side, $100 \mathrm{~m}$. southeast of the Uchinohaye bridge, Ikime machi, Miyazaki gun (N. $31^{\circ} 51^{\prime} 10^{\prime \prime}$, E. $131^{\circ} 19^{\prime} 1^{\prime \prime}$ ).

Fossil bed: Irregularly stratified, grey, hard, calcareous, silty fine sandstone of the upper part of the Tano member.

Occurrence: Rare to common, sporadic.

Cucullaea pamotanensis MARTIN, Vasticardium hyugaense SHUto, Paphia (Paphia) exilis takaokaensis SHUTo, Solen sp., Bathytoma aff. luedorfi (LISCHKE). (23) - Yusunoki-Hashi (MI-1620), northern extremity of the village, road side, 
Takaoka machi, Higashi-Morogata gun (N. $31^{\circ} 53^{\prime} 55^{\prime \prime}$, E. $131^{\circ} 18^{\prime} 53^{\prime \prime}$ ).

Fossil bed: Irregularly stratified, grey, hard calcareous silty fine sandstone with silty matrix of the upper middle part of the Tano member.

Preservation: Moderately favourable, but in general pelecypod dentition is not observable.

Occurrence: Common and sporadic.

Amussiopecten iitomiensis (OTUKA), $\odot$ Vepricardium kyushuense SHUTo, Lucinoma acutilineata (CoNRAD), ○ Paphia (Paphia) exilis takaokaensis SHuto, Clementia (Clementia) papyracea GRAY, Fabulina miyatensis (YoKoyamA), Solecurtes divaricatus LischKe, Siphonalia praedeclivis ITOIGAWA, Fusinus sp.

(24) - Oyamada (MI-3952), road side small cliff, $1100 \mathrm{~m}$. southwest of Oyamada,

Takaoka machi, Higashi-Morogata gun (N. $31^{\circ} 55^{\prime} 45^{\prime \prime}$, E. $131^{\circ} 18^{\prime} 33^{\prime \prime}$ ).

Fossil bed: Grey, hard, calcareous medium sandstone, hardly stratified.

Upper part of the Tano member.

Preservation: Large shells are favourably preserved, but the preservation of the small and medium shells is imperfect.

Occurrence: Common and sporadic.

- Amussiopecten iitomiensis (ОтUKA), Venericardia (Megacardita) megacostata Shuto, Ventricoloidea foveolata miyazakiensis (Shuto), O Paphia (Paphia) exilis takaolaensis SHuto, Cuspidaria (Cuspidaria) hindsiana (A. AdAMs), Brachiopoda gen. and sp. indet.

(25) - Kanekakematsu (MI-3362), small cliff along the ridge $300 \mathrm{~m}$. north of Kanekakematsu, Takaoka machi, Higashi-Morogata gun (N. $31^{\circ} 55^{\prime} 52^{\prime \prime}$, E. $\left.131^{\circ} 17^{\prime} 51^{\prime \prime}\right)$.

Fossil bed: Grey, hard, massive fine sandstone of the upper part of the Tano member; well sorted.

Preservation: Imperfect; shell matter was almost utterly removed. Details of the shell character are not observable.

Occurrence: Rare and sporadic; conjoined valves absent.

Amussiopecten iitomiensis (OTUKA), - Paphia (Paphia) exilis exilis SHuto, $\bigcirc P$. (P.) exilis takaokaensis Shuto, P. (P.) exilis abbreviata SHUto, Eudolium sp. b.

(26)-Kusumi (MI-178), hill side $300 \mathrm{~m}$. northeast of Kusumi, Takaoka machi, Higashi-Morogata gun (N. $31^{\circ} 56^{\prime} 32^{\prime \prime}$, E. $131^{\circ} 17^{\prime} 49^{\prime \prime}$ ).

Fossil bed: Rarely stratified, grey, silty sandstone of the upper part of the Tano member.

Preservation: Favourable.

Occurrence: Common and sporadic; conjoined valves not rare.

$\odot$ Paphia (paphia) exilis exilis SHuto, P. (P.) exilis takaokaensis SHuto.

(27) - Kano (MI-154 and 157), road side small cliff at the southwestern extremity of the village, Takaoka machi, Higashi-Morogata gun (N. $31^{\circ} 56^{\prime} 35^{\prime \prime}$, E. $\left.131^{\circ} 17^{\prime} 33^{\prime \prime}\right)$.

Fossil bed: Scarcely stratified, grey, slightly calcareous silty sandstone of the upper part of the Tano member. 
Preservation: Favourable, but shell matter partially removed. Dentition of the pelecypods is rarely observed.

Occurrence: Common and sporadic; conjoined valves rare.

Nuculana (Nuculana) pennula (YokoYAma), Veletuceta reevei (MAYER), OCrassatellites (Crassatellites) tenuiliratus tenuiliratus SHuto, C. (C.) tenuiliratus triangularis SHUTO, OVenus (Ventricoloidea) foveolata miyazakiensis (Shuto), Paphia (Paphia) exilis exilis Shuto, P. (P.) exilis abbreviata Shuto, $P$. (P.) exilis takaokaensis Shuto, Cantharus sp., Siphonalia praedeclivis IтоGAWA, Parasyrinx n. sp., Bathytoma n. sp.

(28)-Hamako (MI-153), road side near small shrine, Kano, Takaoka machi, Higashi-Morogata gun (N. $31^{\circ} 56^{\prime} 59^{\prime \prime}$, E. $131^{\circ} 17^{\prime} 42^{\prime \prime}$ ).

Fossil bed: Rarely stratified, grey, hard, silty sandstone of the upper part of the Tano member.

Preservation: Imperfect; shell material was partly removed.

Occurrence: Isolated small valves occur sporadically.

Cucullaea pamotanensis MarTiN, Crassatellites (Crassatellites) tosanus NomURA, OPaphia (Paphia) exilis exilis SHuto, Clementia (Clementia) papyracea Gray, Cuspidaria (Cuspidaria) n. sp., C. (Myonella) sp.

(29) - Kawarada (MI-159), road side, 400 m. west of Kawarada, Takaoka machi, Higashi-Morogata gun (N. $31^{\circ} 56^{\prime} 27^{\prime \prime}$, E. $131^{\circ} 11^{\prime} 47^{\prime \prime}$ ).

Fossil bed: Massive, grey, solid sandy siltstone of the upper part of the Tano member.

Preservation: Imperfect, slightly deformed.

Occurrence: Rare and sporadic.

- Acila (Acila) submirabilis Makiyama, Cuspidaria (Cuspidaria) sp.

(30)-Akatani-a (MI-161), road side, eastern extremity of the village, Takaoka machi, Higashi-Morogata gun (N. $31^{\circ} 56^{\prime} 38^{\prime \prime}$, E. $131^{\circ} 16^{\prime} 28^{\prime \prime}$ ).

Fossil bed: Massive, grey, hard, sandy siltstone of the upper part of the Tano member.

Preservation: Favourable, but shell matter was partially removed and the dentition is not always preserved.

Occurrence: Abundant but sporadic; conjoined valves not rare.

Acila (Acila) submirabilis MAKIYAMa, Nuculana (Nuculana) cfr. pennula (YoKOYAMA), Amussiopecten iitomiensis (OTUKA), OJoannisiella cumingii (HANLEY), Pitar (Pitarina) sulfurea PILSBRY, OVenus (Ventricoloidea) foveolata miyazakiensis (Shuto), $\odot$ Paphia (Paphia) exilis exilis Shuto, P. (P.) exilis abbreviata Shuto, P. (P.) hirabayashii tanoensis SHU'T, OClementia (Clementia) papyracea GRAY, Solecurtes divaricatus LischKE, Thracia (Cyathodonta) dissimilis KURODA and HABE, Semicassis japonicus (REEVE), Siphonalia praedeclivis ITOIgaWa.

(31) - Akatani-b (MI-770), road side, at the central part of the village, Takaoka machi, Higashi-Morogata gun (N. $31^{\circ} 56^{\prime} 48^{\prime \prime}$, E. $131^{\circ} 16^{\prime} 13^{\prime \prime}$ ).

Fossil bed: Massive, somewhat calcareous, grey, hard silty sandstone of the upper part of the Tano member; calcareous nodules common. 
Preservation: Moderately favourable; conjoined valves not rare.

Occurrence: Abundant and sporadic.

Amussiopecten iitomiensis (ОTUKA), OCrassatellites (Crassatellites) tenuiliratustenuiliratus SHuTo, C. (C.) tenuiliratus triangularis SHUto, OPitar (Pitar) dohrni (RoEMER), OP. (P.) semeliformis SHuto, OVenus (Ventricoloidea) foveolata miyazakiensis (Shuto), OPaphia (Paphia) exilis exilis Shuto, $P$ (P.) exilis abbreviata Shuto, $P$. ( $P$.) hirabayashii tanoensis Shuto, Clementia (Clementia) papyracea Gray, Solecurtes divaricatus LISC Siphonalia praedeclivis Itolgawa.

(32) - Kawaguchi (MI-766), road side, $400 \mathrm{~m}$. north of the Kawaguchi bridge,

Takaoka machi, Higashi-Morogata gun (N. $31^{\circ} 56^{\prime} 37^{\prime \prime}$, E. $131^{\circ} 15^{\prime} 57^{\prime \prime}$ ).

Fossil bed: Rarely stratified, grey, calcareous fine sandstone with calcareous. nodules; middle part of the Tano member.

Preservation: Favourable; detailed feature of the shells is preserved.

Occurrence: Very rare and sporadic.

- Amussiopecten iitomiensis (OTUKA), Paphia (Paphia) exilis takaokaensis Shuto, Siphonalia (?) sp.

(33)-Yanaze (MI-744), road side, $200 \mathrm{~m}$. east of Yanaze, Takaoka machi, Higashi-Morogata gun (N. $31^{\circ} 56^{\prime} 50^{\prime \prime}$, E. $131^{\circ} 15^{\prime} 31^{\prime \prime}$ ).

Fossil bed: Irregularly stratified, grey, hard, calcareous fine sandstone of the middle part of the Tano member.

Preservation: Moderately favourable.

Occurrence: Dense; partially crowded and partially sporadic.

$\odot$ Amussiopecten iitomiensis (OTUKA), OVenus (Ventricoloidea) joveolata miyazakiensis (Shuto), O Paphia (Paphia) exilis exilis Shuto, $\odot P$. (P.) exilis takaokaensis SHuTo.

(34) - Kurawa-a (MI-451), river side small cliff, along Uranomyo river, $3500 \mathrm{~m}$. northwest of Tanohira, Takaoka machi, Higashi-Morogata gun (N. $31^{\circ} 58^{\prime} 48^{\prime \prime}$, E. $\left.131^{\circ} 12^{\prime} 27^{\prime \prime}\right)$.

Fossil bed: Scarcely stratified, grey, hard sardy siltstone of the upper part of the Tano member.

Preservation: Perfect.

Occurrence: Rare and sporadic; conjoined valves not rare.

- Acila (Acila) submirabilis MAKIYAMA.

(35) - Kurawa-b (MI-458), $3900 \mathrm{~m}$. northwest of Tanohira, Takaoka machi, Higashi-Morogata gun (N. $31^{\circ} 58^{\prime} 19^{\prime \prime}$, E. $\left.131^{\circ} 12^{\prime} 16^{\prime \prime}\right)$.

Fossil bed: Massive, highly calcareous, grey, solid silty sandstone of the upper part of the Tano member.

Preservation: Favourable.

Occurrence: Rare and sporadic.

Chlamys sp., $\odot$ Amussiopecten itomiensi (OTUKA), $\odot$ Paphia (Paphia) exilis takaokaensis SHUTo.

(36) -Waritsuke (MI-671), small cutting, road side $300 \mathrm{~m}$. northeast of Waritsuke, Aya machi, Higashi-Morogata gun (N. $32^{\circ} 0^{\prime} 15^{\prime \prime}$, E. $131^{\circ} 13^{\prime} 43^{\prime \prime}$ ). 
Fossil bed: Rarely stratified, grey, hard, highly calcareous fine sandstone with coarse sand mixture. Upper middle part of the Tano member.

Preservation: Imperfect; shell matter was greatly removed.

Occurrence: Rare and sporadic; conjoined valves rare.

Glycymeris formosana (YoKoYAMA), Veletuceta reevei (MAYER), O Amussiopecten iitomiensis (OTUKA), Ostrea (Pycnodonta) musashiana (YoKoYAl工), Lucinoma sp., Vasticardium hyugaense SHuto, $\odot$ Dosinia (Bonartemis) suketoensis (OtuKa), OPaphia (Paphia) exilis exilis SHUTo, Solecurtes divaricatus LISCHKe, Grammatomya squamosa (LAMARCK).

(37)-Mokudô (MI-437), river side cliff, Aya machi, Higashi-Morogata gun (N. $32^{\circ} 0^{\prime} 57^{\prime \prime}$, E. $\left.131^{\circ} 14^{\prime} 17^{\prime \prime}\right)$.

Fossil bed: Solid, massive, granule conglomerate cemented by calcareous matter. Lower part of the Tano member.

Preservation: Imperfect, slightly worn.

Occurrence: Common and sporadic.

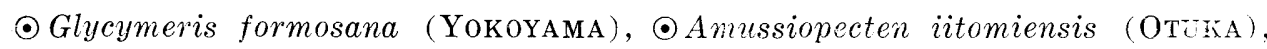
Ostrea (Nodostrea) sp., Trachycardium cfr. shiobarense (YokоYAмA), Paphia (Paphia) hirabayashii tanoensis SHUTo.

(38)-Kugino (MI-565), road side cutting, $900 \mathrm{~m}$. south of Kugino, Aya machi, Higashi-Morogata gun ( N. $32^{\circ} 1^{\prime} 11^{\prime \prime}$, E. $131^{\circ} 15^{\prime} 29^{\prime \prime}$ ).

Fossil bed: Scarcely stratified, grey, brittle, slightly calcareous silty fine sandstone of the uppermost part of the Tano member.

Preservation: Perfect.

Occurrence: Abundant and sporadic; conjoined valves common.

$\bigcirc$ Acila (Acila) submirabilis MAKIYAMA, $\bigcirc$ Glycymeris rotunda DUNKER, Limopsis obliqua A. ADAMs, Atrina (Servatrina) pectinata (LINNÉ), OJoannisiella cumingii (HANley), • Paphia (Paphia) hirabayashii loyuensis SHuto, O Polynices (Glossaulax) reiniana (DUNKER) subsp., Fulgoraria (Psephaea) liosibensis OTUKA.

(39) - Nakabyu-a (MI-2019), river side near bridge, Yatsushiro mura, Koyu gun (N. $32^{\circ} 2^{\prime} 58^{\prime \prime}$, E. $\left.131^{\circ} 17^{\prime} 49^{\prime \prime}\right)$.

Fossil bed: Rarely stratified, grey, hard, somewhat calcareous silty sandstone with coarse sand mixture Upper part of the Kawabaru member.

Preservation: Moderately favourable.

Occurrence: Rare and sporadic.

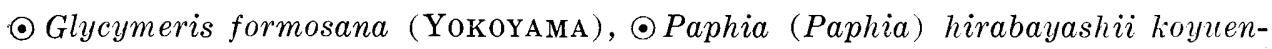
sis Shuto, ODosinia (Phacosoma) nomurai (OTUKa).

(40) - Nakabyu-b (MI-2017), $200 \mathrm{~m}$. east of the village, road side, Yatsushiro mura, Koyu gun (N. $32^{\circ} 2^{\prime} 53^{\prime \prime}$, E. $131^{\circ} 18^{\prime} 2^{\prime \prime}$ ).

Fossil bed: Grey, brittle, massive siltstone overlying the fossil horizon of the preceding one.

Preservation: Favourable.

Occurrence: Sporadic and rare. 
$\odot$ Acila (Acila) submirabilis MAKIYAma, $\odot$ Limopsis obliqua A. AdAms, Nuculana. sp.

(41) - Kakoi (MI-4074), brook side $350 \mathrm{~m}$. south of Kakoi village, $100 \mathrm{~m}$. east of the highway, Sanzai mura, Koyu gun (N. $32^{\circ} 3^{\prime} 58^{\prime \prime}$, E. $131^{\circ} 18^{\prime} 55^{\prime \prime}$ ).

Fossil bed: Poorly stratified, grey, hard, calcareous silty sandstone of the upper part of the Kawabaru member.

Preservation: Almost perfect; shell matter is preserved in favourable condition to enable the scrutiny of the pelecypod dentition.

Occurrence: Abundant, but sporadic.

OAcila (Acila) submirabilis MAKIYAMA, Limopsis obliqua A. ADAM, Gloripallium miurensis (YокоҮАма), Crassatellites (Crassatellites) tosanus NomuRA, OJoannisiella cumingii (HANLEY), Thyasira (Conchocele) cfr. nipponica YABE and Nomura, $\odot$ Nemocardium (Keenaea) samarangae (MAKIYAMA), Vepricardium kyushuense SHuto, Macoma (Pseudometis) praerupta (SALISBURY), Cuspidaria (Cuspidaria) sp. b., Lischkeia alwinae (LISCHKE), Suchium (Protorotella) maxima SHuto, Tectonatica janthostomoides KURODA and HABE, Eudolium oyamai Shuto, Galeodea n. sp., Murex (Murex) cfr. djarianensis MarTin, Nassaria magnifica LischKe subsp., Granulifusus koyuanus SHUTo, Columbarium pagodum (LESSON), Ancilla (Turrancilla) miserula (Yokoyama), Cancellaria cfr. pristina (Yokoyama), Ancistrosyrinx osawanoensis Tsuda Spirotropis subdeclivis (YokoYAMA) n. subsp., Ringicula sp.

(42)-Kanekura (MI-4082), river side small cliff $200 \mathrm{~m}$. down stream from the bridge at Kanekura, Sanzai mura, Koyu gun (N. $32^{\circ} 4^{\prime} 12^{\prime \prime}$, E. $131^{\circ} 19^{\prime} 39^{\prime \prime}$ ).

Fossil bed: Rarely stratified, grey, brittle siltstone of the uppermost part of the Kawabaru member.

Preservation: Slightly deformed, but shell matter is perfectly preserved.

Occurrence: Very rare and sporadic.

Limopsis obliqua A. AdAms, $\odot$ Ancilla (Turrancilla) otukai SHuto, Fulgoraria (Psephaea) sp.

(43) - Azukino-a (MI-4853), brook side small cliff, $300 \mathrm{~m}$. northeast from the extremity of the village, Sanzai mura, Koyu gun (N. $32^{\circ} 4^{\prime} 48^{\prime \prime}$, E. $131^{\circ} 19^{\prime} 37^{\prime \prime}$ ). Fossil bed: Calcareous nodules intercalated in the grey, brittle fine sandstone in the irregularly alternating sequence of sandstones and siltstones of the upper part of the Kawabaru member.

Preservation: Shells are generally in favourable state of preservation, but they are tend to be broken in taking out from the rock because of tight cementation.

Occurrence: Dense and sporadic.

Acila (Acila) submirabilis VĩakiYama, Ennucula sp., O Saccella confusa kongiensis OTUKa, Anadara (Scapharca) sp., OGlycymeris formosana (YoKoYama), Crassatellites (Crassatina) oblongatus uchidanus (YokoYama), OJoannisiella cumingii (HANLEY), Vepricardium kyushuense Shuto, ONemocardium (Keenaea) samarangae (MakiYama), Callista (Callista) chinensis (Holten), Pitar (Pitarina) pellucida (LAMARCK), Dosinia (Phacosoma) nomurai OTUKA, Mer- 
cenaria chitaniana (YokоYама), Crepidula sp., Xenophora (Tugurium) dunkeri Martin, Nassaria magnifica (LischKe) subsp., Granulifusus koyuanus SHUto, Murex (Murex) cfr. verbecki MARTIN, Fasciolaria sp., Spirotropis subdeclivis (Yokoyama) n. subsp., Asprella (Endemoconus) toyamaensis TsudA, Ringicula. sp.

(44)-Azukino-b (MI-4862), $60 \mathrm{~m}$. apart the preceding locality, Sanzai mura, Koyu gun (N. $32^{\circ} 4^{\prime} 42^{\prime \prime}$, E. $\left.131^{\circ} 19^{\prime} 11^{\prime \prime}\right)$.

Fossil bed: Stratified, grey, hard, sandy siltstone of the upper part of the Kawabaru member.

Preservation: Perfect.

Occurrence: Few and sporadic; conjoined valves common.

$\odot$ Limopsis obliqua A. AdAMs, Lima (Acesta) goliath (SowerBy), Cuspidaria (Cuspidaria) n. sp., C. (Cardiomya) sp., $\odot$ Spirotropis subdeclivis (YoкоYAмA) n. subsp.

(45) - Nagano (MI-5109), road side, south end of the village, Mino mura, Koyu gun (N. $32^{\circ} 6^{\prime} 26^{\prime \prime}$, E. $131^{\circ} 21^{\prime} 55^{\prime \prime}$ ).

Fossil bed: Scarcely stratified, grey, brittle sandy siltstone of the uppermost part of the Kawabaru member.

Preservation: Moderately favourable, but slightly deformed.

Occurrence: Rare and sporadic; conjoined valves common.

- Parvamussium (Parvamussium) kyushuense SHuto, Cuspidaria (Cuspidaria) hindsiana (A. AdAMs), Fulgoraria (Psephaea) sp.

(46) - Yamaji-a (MI-5060 to 5061), road side, between the village and the tunnel, Mino mura, Koyu gun (N. $32^{\circ} 7^{\prime} 17^{\prime \prime}$, E. $131^{\circ} 22^{\prime} 26^{\prime \prime}$ ).

Fossil bed: Irregularly stratified, grey, hard sandy siltstone with some calcareous nodules of the uppermost part of the Kawabaru member. Two localities (MI-5060 and 5061) apart about $150 \mathrm{~m}$. each other, but belong to the same bed of about $3 \mathrm{~m}$. thick.

Preservation: Generally favourable and the detailed characteristics are observable.

Occurrence: Abundant and sporadic except in the calcareous nodules which bear crowded molluscan specimens and plant leaves such as Quercus.

○ Acila (Acila) submirabilis MAKIYAMA, Ennucula cfr. akitana OTUKA, O Saccella. confusa kongiensis OTUKA, OYoldia (Tepidoleda) naganumana OTUKA n. subsp., Acar plicata (DILWYN), Glycymeris rotunda DUNKER, OLimopsis obliqua A. AdAms, Pectunculina oblonga (A. AdAms), Lima (Acesta) goliath (Sowerby), Nemocardium (Keenaea) samarangae (MAKIYAMA), Lucinoma annulata (REEve), Dosinia (Phacosoma) cfr. nomurai OTUKA, Lischkeia alwinae (LISCHKE), Galeodea n. sp., Siphonochelus (Siphonochelus) japonica (A. ADAMS), Fulgoraria (Fulgoraria) daviesi (FultoN), F. (Psephaea) sp., Uromitra nakamurai ugariensis MAKIYAMA, Inquisitor (Inquisitor) hyugana (YoKOYAMA) n. subsp., Turricula (Turricula) n. sp., Bathytoma n. sp., Spirotropis subdeclivis (YокоYама), O Turris (Gemmula) n. sp., Ptychosyrinx n. sp., Clavatula (Paradrillia) n. sp. 
(47) - Yamaji-b (MI-5070), cutting approach to the tunnel, Mino mura, Koyu gun (N. $32^{\circ} 7^{\prime} 20^{\prime \prime}$, E. $\left.131^{\circ} 22^{\prime} 33^{\prime \prime}\right)$.

Fossil bed: Grey, brittle, fully sorted fine sandstone intercalated in the irregularly stratified sandy siltstone sequence of the uppermost part of the Kawabaru member.

Preservation: Perfect.

Occurrence: Abundant but not crowded.

Glycymeris rotunda Dunker, Pteria sp., Chlamys sp., Scambra (Eucrassatella) sp., Crassatellites (Crassatellites) tsumaensis Sнuтo, Venericardia (Megacardita) megacostata SHUTO, Joannisiella cumingii (HANLEY), Nemocardium (Keenaea) samarangae (MAKIYAMA), Vepricardium kyushuense SHUTo, Clinocardium subdecussatum Shuto, Callista (Callista) roscida Gould, Paphia (Paphia) hirabayashii koyuensis Shuto, P. (P.) tsumaensis Shuto, Clementia (Clementia) papyracea Gray, Placamen tiara (DILWYN), Dosinia (Phacosoma) nomurai OTUKA, Solen sp., Solecurtes dunkeri Kuroda, Fabulina nitidula (DuNkeR), Periploma pulchellum HATAI and Nisiyama, Cadella semitorta (Sowerby), Myadora proxima SMITH, Cuspidaria (Cardiomya) n. sp., Calliostoma (Otukaia) lounjianum (Yoкочама), Suchium (Protorotella) hyugaensis Shuto, $S$. (Suchium) koyuense SHuto, S. (S.) n. sp., Polinices (Glossaulax) reiniana (DUNKer) subsp., Mammila simiae (DEshayes), Tectonatica janthostomoides Kuroda and HABE, Tonna costatum (DEshaYes), Eudolium oyamai Shuto, Pirula sp., Cymatium cfr. tenuiliratus (LISCHKE), Siphonalia tonohamaensis totomiensis MaKIYAma, Nassaria magnifica (Lischke) subsp., Plicifus? sp., Nassarius (Hinia) caelatus (A. ADAMS) n. subsp., Granulifusus koynanus Shuto, Oliva (Oliva) ispidula LinNé, Olivella (Olivella) fulgurata (AdAMs and REEVE), O. (O.) consobrina (LischKe), Retusa sp.

(48) - Shimauchi (MI-4912), bank of the pond, south of Minamikata, Saito City (N. $32^{\circ} 7^{\prime} 33^{\prime \prime}$, E. $\left.131^{\circ} 23^{\prime} 7^{\prime \prime}\right)$.

Fossil bed: Irregularly stratified, grey, slightly calcareous sandy siltstone of the uppermost part of the Kawabaru member.

Preservation: Favourable.

Occurrence: Rare and sporadic.

$\odot$ Acila (Acila) submirabilis MakiYama, ๑Limopsis obliqua A. AdAms, OUberella yokoyamai KURODA and HABE.

(49)-Kushiki (MI-4923), road side, $250 \mathrm{~m}$. north of the bridge, Kamihokita mura, Koyu gun (N. $32^{\circ} 8^{\prime} 31^{\prime \prime}$, E. $\left.131^{\circ} 23^{\prime} 29^{\prime \prime}\right)$.

Fossil bed: Irregularly stratified, grey, brittle sandy siltstone of the upper part of the Kawabaru member.

Preservation: Generally favourable, but in some specimens shell matter was removed greatly.

Occurrence: Rare and sporadic.

Acila (Acila) submirabilis MAKIYAMA, Cucullaea granulosa (JoNAs), OGlycymeris formosana (YoKoYAMA), ONemocardium (Keenaea) samarangae (MAKIYAMA), Paphia (Paphia) hirabayashii koyuensis Sнuto, Cuspidaria (Myonella) 
n. sp., Fulgoraria (Psephaea) kosibensis OTUKA, Borsonia sp., Surculites siogamensis Nomura.

(50)-Takajô (MI-5377), river side cliff, Kijô mura, Koyu gun (N. $32^{\circ} 9^{\prime} 35^{\prime \prime}$, E. $\left.131^{\circ} 28^{\prime} 24^{\prime \prime}\right)$.

Fossil bed: Scarcely stratified, grey, brittle, silty sandstone of the upper part of the Kawabaru member.

Preservation: Perfect.

Occurrence: Common and sporadic.

$\odot$ Acila (Acila) submirabilis MakiYama, $\odot$ Merisca serricostata (TokunAGa), Ancilla (Baryspira) albocallosa okawai (YokoyAMA), Clavatula sp., Inquisitor (Inquisitor) jeffreysii (SMITH) n. subsp., Thatcheria gradata (YoKoYAMA).

(51) - Jinkundani (MI-5392), east cliff at the entrance of Jinkundani dale, Kijô mura, Koyu gun (N. $32^{\circ} 9^{\prime} 46^{\prime \prime}$, E. $131^{\circ} 27^{\prime} 26^{\prime \prime}$ ).

Fossil bed: Grey, brittle, fine sandstone with coarse sand patches intercalated in the irregularly stratified medium sandstones of the upper middle part of the Kawabaru member.

Preservation: Imperfect; shell surface is moderately worn.

Occurrence: Abundant and sporadic.

$\odot$ Glycymeris formosana (YoKoYAMA), ๑ Paphia (Paphia) hirabayashii koyuensis Shuto, Tectonatica janthostomoides KURoDA and HABE.

(52)-Yamasumi (MI-5135), river side cliff east of the bridge, Tsuma, Saito City (N. $32^{\circ} 6^{\prime} 37^{\prime \prime}$, E. $\left.131^{\circ} 25^{\prime} 19^{\prime \prime}\right)$.

Fossil bed: Rarely and obscurely stratified grey, brittle siltstone of the middle part of the Tsuma member.

Preservation: Favourable; minute structures are preserved.

Occurrence: Rare and sporadic; conjoined valves common.

OMacoma (Pseudometis) praerupta (SALISBURY), OPeriploma besshoensis (YoKoYama) n. subsp., $\odot$ Operculina ammonoides (GronoviUs).

(53) - West of Nishinobyu (MI-5541), $600 \mathrm{~m}$. west of the village, hill side, Kawaminami mura, Koyu gun (N. $32^{\circ} 9^{\prime} 44^{\prime \prime}$, E. $131^{\circ} 27^{\prime} 47^{\prime \prime}$ ).

Fossil bed: Scarcely stratified, pale grey, brittle sandy siltstone of the upper part of the Tsuma member.

Preservation: Favourable.

Occurrence: Rare and sporadic; almost all the pelecypod specimens are conjoined.

Thyasira n. sp., OMacoma (Pseudometris) praerupta (SALISBURY), O Periploma besshoensis (YoкоYама) n. subsp., Mangelia sp.

(54)--Tonogôri (MI-4717), north hillside of Tonogôri, Saito City (N. 32 $3^{\prime} 49^{\prime \prime}$, E. $\left.131^{\circ} 22^{\prime} 42^{\prime \prime}\right)$

Fossil bed: Thin, grey, calcareous fine sandstone intercalated in the monotonous grey, brittle siltstone sequence of the Tonogôri member.

Preservation: Imperfect; shell matter has been greatly removed in the course of weathering, though the original state is favourable.

Occurrence: Abundant and crowded. 
Acila (Acila) submirabilis MakiYama, Pseudogrammatodon sp., Barbatia (Barbatirus) cometa (REEVE), OGlycymeris rotunda DUNKER, OG. totomiensis MakiYama, Limopsis obliqua A. Adams, Amusium n. sp., Mimachlamys miniacea (LAmarck), Ostrea (Pycnodonta) musashiana (Yokoyama), Crassatellites (Crassatellites) tosanus NomURA, OVenericardia (Megacardita) oyamai SHUTo, OJoannisiella cumingii (HANLEY), Thyasira tokunagai KurodA and HABE, T. sp., Felaniella usta (GoULD), Lucinoma annulata (REEVE), OPillucina pisidium (DUNKer), P. (Sydlorina) cfr. yokoyamai OTUKA, ONemocardium (Keenaea) samarangae (MAKIYAMA), Vepricardium kyushuense Shuto, Paphia (Paphia) grata tsumaensis Shuto, Callista (Callista) chinensis (Holten), Dosinia (Phacosoma) cfr. nomurai OTUKA, Merisca cfr. serricostata (ToKUnAGA), Angulus sp., Dentalium hexagonum Gould, Siphonodentalium (Pulserum) ozawai (YokoYAMA), "Dentalium" sp., Ethalia (Ethaliella) ? sp., Calyptraea sp., Crepidula cfr. isiiana OTUKA, Turritella perterebra Yoкоуама, Xenophora (Tugurium) dunkeri MARTIN, Polinices (Glossaulax) reiniana (DUNKER) subsp., OUberella yokoyamai KURODA and HABE, Eudolium oyamai SHUTo, E. sp. a, Tonna deshayesii (REeve), Apollon sp., Murex (Murex) cfr. verbecki MaRTin, Columbarium pagodum (LESSON), Mitrella sp., Kelletia sp., Siphonalia declivis tosaensis MAKIyAMA, S. dainitiensis MAKIYAMA, S. ikebei TsudA, Babylonia areolata LINNÉ, Cantharus wangwanensis NomURA, ONassaria magnifica (LISCHKE) n. subsp., Buccinum sp., Nassarius sp., Granulifusus dualis (YокоYамA), Oliva (Oliva) mustellina LAMARCK, Ancilla (Baryspira) albocallosa okawai (YoKoYAMA), Olivella (Olivella) fulgurata (ADAMS and REEVE), Sydaphera spengleriana (DEshayes), Cancellaria (Merica) cfr. pristina (Yokoyama), Turris cfr. kurodai MaKIYAMa, O Turricula (Surcula) sobrina (YoKoYama), Ancistrosyrinx osawanoensis TsUDA, Bathytoma sp., OInquisitor (Inquisitor) jeffreysi (E. A. SMITH), Turricula spp., Retusa sp.

(55) - Kirishima (MI-4690), foot of the hill $650 \mathrm{~m}$. south of Kirishima, Tonogôri, Saito City (N. $32^{\circ} 4^{\prime} 19^{\prime \prime}$, E. $131^{\circ} 23^{\prime} 12^{\prime \prime}$ ).

Fossil bed: Coarse sand bearing grey, hard siltstone intercalated in the alternation sequence of medium sandstones and siltstones of the Tonogôri members.

Preservation: Moderately favourable.

Occurrence: Generally rare but somewhat crowded in thin layers and patches.

$\odot$ Glycymeris rotunda (DUNKer), OG. totomiensis MAKIYAMA, Polinices (Glossaulax) reiniana (DUNKER) subsp.

(56)-Kubo (MI-310), $400 \mathrm{~m}$. southwest of Kubo on the road from Kubo to

Uyeno, Uryuno, Miyazaki City (N. $31^{\circ} 59^{\prime} 54^{\prime \prime}$, E. $131^{\circ} 24^{\prime} 18^{\prime \prime}$ ).

Fossil bed: Pale grey, hard siltstone in the alternation sequence of medium sandstones and siltstones of the Uryuno member.

Preservation: Moderately favourable.

Occurrence: Rare and sporadic.

$\odot$ Glycymeris rotunda DUNKER, G. totomiensis MAKIYAMA, Limopsis obliqua A. 
AdAms, Polnices (Glossaulax) reiniana (DUnker) subsp., Ancilla (Turrancilla) miserula (YoKоYAMA).

(57) -Kôonji (MI-5528), road side cutting $400 \mathrm{~m}$. southwest of Kôonji, Takanabe machi, Koyu gun (N. $32^{\circ} 6^{\prime} 45^{\prime \prime}$, E. $131^{\circ} 30^{\prime} 17^{\prime \prime}$ ).

Fossil bed: Grey, hard, somewhat calcareous siltstone intercalated in the irregular alternation of sandstones and siltstones of the lower part of the Takanabe member.

Preservation: Perfect; minute structures of the shell are preserved.

Occurrence: Rare and sporadic; conjoined valves common.

- Acila (Acila) submirabilis MAKIYAMA, Yoldia (Tepidoleda) naganumana (OTUKa) n. subsp., Uberella cfr. yokoyamai KuRoda and HABE.

(58) - Hagenoshita (MI-6351), road side cutting on the highway south of Hagenoshita, Uwaye mura, Koyu gun (N. $32^{\circ} 8^{\prime} 35^{\prime \prime}$, E. $131^{\circ} 30^{\prime} 57^{\prime \prime}$ ).

Fossil bed: Unconsolidated grey, fine sand with brownish tint and the underlying grey, brittle sandy siltstone of the lower part of the Takanabe member.

Preservation: Perfect in sand and siltstone.

Occurrence: Abundant but not crowded; pelecypods valves are generally separated, but not at all abraded.

(in siltstone)

Limopsis obliqua A. ADAMs, Erodona cfr. amurensis SCHRENCK, OOrectospira excelsa (YoKоYAмA), OPolinices (Glossaulax) reiniana (DUNKER) subsp., O Nassaria lischkei MakiYama, N. magnifica Lischke, Fulgoraria (Psephaea) kamakuraensis OTUKA, Lyria mizuhonica koyuana (YокоYамA), Bathytoma striatotuberculata (YokoYAma), OLencosyrinx coreanica (AdAMs and REEVE), Cymatosyrinx osawanoensi TsudA n. subsp., Pseudoinquisitor totomiensis ugariensis MAKIYAMA.

(in sand)

Anadara (Diluvarca) tricenicosta (NYST), Hawaiarca uwaensis (YoKoYAMA), OGlycymeris rotunda DUNKeR, G. totomiensis MAKIYAMA, Limopsis obliqua A. Adams, Pectunculina oblonga (A. Adams), Oblimopa forskalii (A. AdAms), Cryptopecten vesiculosus (DUNKeR), Anomia lischkei DAUTZENBERG and Fischer, Crassatellites (Crassatellites) tosanus Nomura, Venericardia (Magacardita) granulicostata Nomura, $\bigcirc V$. (M.) panda (YoKoyama), Nemocardium (Keenaea) samarangae (MAKIYAMA), Callista (Callista) chinensis (HoLTeN), Veremolpa scabra (HANLEY), OMyadora proxima (SMITH), Dentalium hexagonuum Gould, D. (Antalis) totomiensis MAKIYAMA, Siphonodentalium (Pulserum) ozawai (Yoкочама), Calliostoma (Otukaia) kounjianum (Yoкоуама), Suchium (Suchium) obsoletum arenarium MakiYAma, Starkeyna pusillum (C. B. AdAMS), S. sobrina (A.ADAMS), Calyptraea yokoyamai Kuroda, Crepidula cfr. isimotoi OTUkA, Turritella (Haustator) n. sp., ○Orectospira excelsa (YokoAma), Solarium (Philippia) cingulum (KIENER), Bittium binodulosum YoKoAMA, Melanella (Balcis) musta (Yoкоуама), Pyramidella (Tibera) spp., Turbonilla (Tropaeas) sp., T. (Turbonilla) spp., Odostomia (Odostomia) hilgendorfi (Lesson), O. (O.) 
spp., Syrnola sp., Kleinella (Leucotina) sp., Menestho (Oscilla) sp., Uberella rufa (BORN), U. yokoyamai KURODA and HABE, Tectonatica janthostomoides KuRODA and HABE, T. andoi NomURA, O Polinices (Glossaulax) reiniana (DUNKER) subsp., P. (G.) didyma (RöDING), P. (G.) didyma (RödING) subsp., Mammila simiae (DeshyaEs), Volva sp., Cymatium cfr. exaratus (REeve), Apollon bituberculata (LAMARCK), Murex (Murex) djarianensis MARTIN, Ocenebra adunka (SowerBY), Poirieria azami KURODA, Chicoreus pliciferoides KURODA, Mitrella bicinctella YoKOYAMa, M. lischkei SMITh, OSiphonalia declivis declivis Yokoyama, S. declivis tosaensis Yokoyama, $S$. declivis Yokoyama n. subsp. a. S. declivis Yokoyama n. subsp. b, S. spadicea fuscolineata (PEASE), Babylonia elata (YoKoYAMA), ๑ Nassaria lischkei MAKIYAMA, N. magnifica LisCHKE, N. magnifica LischKe subsp., Hemifusus ternatanus (GMELIN), Nassarius (Hinia) festivus (PowYs), Granulifusus dualis (YokоYAмa), G. matsumotoi SHuto, Oliva (Oliva) mustellina LAMARCK, Ancilla (Baryspira) albocallosa okawai (Yokoyama), A. (B.) oyamai Shuto, OOlivella (Olivella) spretoides Yokoyama, OO. (O.) baetica CARPENTER, Fulgoraria (Fulgoraria) cancellata KURODA and HABE, Cancellaria (Merica) pristina (Yokoyama), C. (Cancellaria) reevei laticostata (LOEBECKe), Sydaphera cfr. spengleriana (DEshaYes), Trigonostoma kurodai MAKIYAMA, Mitra hanlayana DUNKER, Fuscomitra sp., OMarginella novemprovincialis (YoKoYAMA), $M$. flaccida (Yokoyama), OTurris kurodai MAKIYAMA, Gemmula granosa (HelBRING) n. subsp., Optoturris n. sp., Ptychosyrinx n. sp., Bathytoma striatotuberculata (Yокочама), B. luedorf (LISCHE), OSpirotropis subdeclivis (YoKoYAMA), Leucosyrinx coreanica (ADAMS and REEVE), Turricula (Orthosurcula) pervirgo (Yокочама), T. (Surcula) sobrina (Yoкоуама), T. (S.) n. sp., Ancistrosyrinx osawanoensis TSUDA, Cosmasyrinx n. sp., Cymatosyrin $x$ osawanoensis TSUDA n. subsp., C. rinsuikowaensis NomURA, Clavatula (Paradrillia) djocdjocartae serrana (FISCHER), C. (P.) astuta (YoKoYAMA), C. (P.) dainichiensis (YokоYama), Inquisitor (Inquisitor) jeffreysii (SмITH), Pseudoinquisitor totomiensis ugariensis MAKIYAMA, Tomopleura subdifficilis (MAKIYAMA), $T$. yokoyamai (MAKIYAMA), Etrema hayasakai Nomura, Lienardia (L.) n. sp., Micantapex n. sp., Borsonia n. sp., B. sp., Comitas sp., Propebella cfr. yokoyamai Ozaki sp., Asprella tomacosa (PILsBRY), Filodrillia n. sp., Antiguraleus sp., Strioterebrum (Punctoterebra) makiyamae Tsuda, S. (P.) ozawai Yokoyama, Myurella (Pervicacia) recticostata (YoKoyama), "T." spp., Ringicula (Ringiculina) teramachii HABE, $R$. (R.) doliaris (GoUld), Acteocina oyamai HABE.

(59) - Nishinobyu (MI-5553), hill side east of the village, Kawaminami mura, Koyu gun (N. $32^{\circ} 9^{\prime} 32^{\prime \prime}$, E. $\left.131^{\circ} 30^{\prime} 1^{\prime \prime}\right)$.

Fossil bed: Rarely stratified, pale grey, brittle siltstone of the lower part of the Takanabe member.

Preservation: Perfect.

Occurrence: Very rare and sporadic.

$\bigcirc$ Limopsis obliqua A. AdAMs, Cymatosyrinx rinsuikawaensis Nomura, Propebella cfr. yokoyamai OzAKI. 
(60)-Nihonmatus (MI-5551), road side, Takanabe machi, Koyugun (N. $32^{\circ} 6^{\prime} 42^{\prime \prime}$, E. $\left.131^{\circ} 31^{\prime} 17^{\prime \prime}\right)$.

Fossil bed: Unconsolidated, grey, thin, fine sandstone intercalated in the pale grey siltstone sequence of the middle part of the Takanabe member.

Preservation: Perfect.

Occurrence: Abundant; generally sporadic, but partially crowded in thin layers or patches. Large fossil absent.

Nucula (Nucula) paulula (A. ADAMS), Glycymeris rotunda DUNKER, Limopsis obliqua A. Adams, Pectunculina oblonga (A. ADAms), Anomia sp., Bellucina civica (YoKoYAMA), Joannisiella lunaris (YoKoYAMA), Myadora proxima (SмIтH), Pseudoliotia micans (A. ADAMs), Turritella perterebra YoKoYAma, Orectospira excelsa (YoKoYama), Bittium binodulosum YoKoYama, Turbonilla sp., Melanella (Balcis) musta (Yokoyama), Polinices (Mammilaria) sagamiensis (Plisbry), P. (Glossaulax) didyma (RöDING) subsp., O Uberella yolkoyamai KURODA and HABE, $U$. n. sp., Tectonatica janthostomoides KuRODA and HABE, Cymatium eñr. tenuiliratus (LISCHKE), Chicoreus pliciferoides KURODA, Siphonalia declivis declivis Yokoyama, S. declivis tosaensis MakiYama, ONassaria lischkei MAKIYAMA, Babylonia elata (YoKoYAMA), OGranulifusus dualis (YoKoYama), G. matsumotoi Shuto, Nassarius (Hinia) caelatus (A. AdAMs) n. subsp., N. (H.) caelatus dainitiensis MAKIYAMA, Ancilla (Baryspira) albocallosa okawai (YoKoYAMA), Olivella (Olivella) spretoides YoKOYAMA. OMarginella novemprovincialis (Yokoyama), M. flaccida (Yokoyama), Gemmula graiosa (Helbring) n. subsp., O Bathytoma striatotuberculata (YokoYAMA), O Spirotropis subdeclivis (YoKoYAMA), Leucosyrinx coreanica (A. ADAMS and REEVE), Clavatula (Paradrillia) dainichiensis (Yokоуама), Tomopleura subdifficilis MaкiYama, Cymatosyrinx rinsuitawaensis NomURA, Bellaspira (Lyromangilia) semicarinata (PILSBRY), Acteon kirai HABE, Pupa strigosa (GOULD), Ringicula (Ringiculina) doliaris (GOULD).

(61) - Idenouye (MI-5669), river side small cliff south of Idenouye, Kawaminami machi, Koyu gun (N. $32^{\circ} 12^{\prime} 53^{\prime \prime}$, E. $131^{\circ} 31^{\prime} 38^{\prime \prime}$ ).

Fossil bed: Pale grey, rather soft fine sandstone intercalated in the silty sequence of the middle part of the Takanabe member.

Preservation: Favourable.

Occurrence: Sporadic; conjoined valves not rare.

- Cucullaea granulosa (JoNAs), Limopsis obliqua A. ADAMs.

(62)-Iyedoko (MI-5575), cliff at the entrance of a small lane southwest of Shigino village, Uwaye mura, Koyu gun (N. $32^{\circ} 8^{\prime} 30^{\prime \prime}$, E. $131^{\circ} 31^{\prime} 54^{\prime \prime}$ ).

Fossil bed: Irregularly stratified, pale grey silty sandstone of the upper middle part of the Takanabe member.

Preservation: Moderately favourable.

Occurrence: Rare and sporadic.

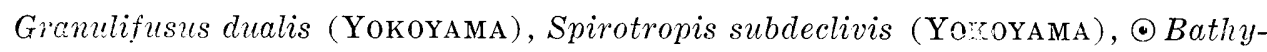
toma striatotuberculata (YoKoYAMA).

(63)-Kizukume (MI-5739), upper part of the south cliff of the hill at Kizukume, 
Tonda machi, Koyu gun (N. $32^{\circ} 4^{\prime} 12^{\prime \prime}$, E. $131^{\circ} 30^{\prime} 21^{\prime \prime}$ ).

Fossil bed: Moderately weathered, brownish grey, tuffaceous, rigid silty sandstone intercalated in the tuffaceous sandy siltstone sequence of the upper part of the Takanabe member.

Preservation: Perfect; minute feature of the shell is observable.

Occurrence: Abundant, but not crowded.

Acila (Truncacila) minuta (MakiYama), Acila (Acila) divaricata (Hinds), Limopsis obliqua A. ADAMs, Gloripallium aurantiacus (ADAMS and REEvE), Cryptopecten vesiculosus (DUNKER), Palliolum (Delectopecten) macrocheiricola HABE, Anomia sp., OCrassatellites (Crassatellites) takanabensis SHuto, $\odot$ Nemocardium (Keenaea) samarangae (MAKIYAMA), Dosinia (Phacosoma) troscheli (LISchKe), Veremolpa mindanensis (SMITH), Solecurtes dunkeri KuRoda, Mysella n. sp., Turcicula argenteonitens convexiuscula (Yokoyama), Turcica sp., Polinices (Glossaulax) reiniana (DUNKER) subsp., Mitrella bicinctella (YoKoYAMA), Siphonalia mikado Melville, Nassaria magnifica Lischke subsp., Granulifusus dualis (YokоYамa), G. matsumotoi ShUto, Fusinus simplex SMITH, Turris kurodai MaKiYama, Gemmula granosa (Helbling), Bathytoma striatotuberculata (YokоYама), Spirotropis subdeclivis (YокоYамA), OLeucosyrinx coreanica (AdAMs and ReEve), Turricula (Surcula) sobrina (YoKoYAma).

(64) - Iwawaki (MI-5674), small cliff at the south extremity of the hill, Tonda machi, Koyu gun (N. $32^{\circ} 4^{\prime} 30^{\prime \prime}$, E. $\left.131^{\circ} 30^{\prime} 39^{\prime \prime}\right)$.

Fossil bed: Shell bank in the tuffaceous sitly sandstone of the upper part of the Takanabe member.

Preservation: Perfect; but the detouched specimens were more or less broken in separating from the rock because of the tight cementing.

Occurrence: Shell bank; greater parts of the constituents are aberrant forms of Lucinoma annulata.

Glycymeris totomiensis MAKIYAMA, Pectunculina oblonga (A. ADAMS), Venericardia (Megacardita) ferruginosa (A. ADAMS and REEVE), O Nemocardium (Keenaea) samarangae (MAKIYAMA) [aberrant form], - Lucinoma annulata (REEve) [aberrant form], Trochus sp., Calliostoma (Tristichotrochus) shinagawaense cipangoanum (YoKoYAMA), Xenophora (Tugurium) dunkeri MaRTIN.

(65)-Tôriyama (MI-5595), cliff at the entrance of a gorge $1200 \mathrm{~m}$. south of

Tôriyama-Hama, Kawaminami machi, Koyu gun (N. $32^{\circ} 9^{\prime} 40^{\prime \prime}$, E. $131^{\circ} 32^{\prime} 45^{\prime \prime}$ ).

Fossil bed: Rarely stratified, pale grey, rigid, somewhat calcareous, tuffaceous silty sandstone of the upper part of the Takanabe member.

Preservation: Perfect.

Occurrence: Abundant, but not crowded; conjoined valves common. OAnadara (Scapharca) castellata (YokoyAMA), A. sp., O Glycymeris rotunda Dunker, Veletuceta nakamurai (MakiYama), Limopsis obliqua A. AdAMs, Pectunculina oblonga (A. AdAMs), Volsella nitida (REEve), Atrina penna (REEVE), Gloripallium satowi (YoKoyama), G. aurantiacus (Sowerby), Mimachlamys miniacea (LAMARCK), Cryptopecten vesiculosus (DUNKER), Patinopecten taiwanus Nomura, $\bigcirc$ Amussiopecten praesignis (YoKoYAMA), Ostrea (Pycnodonta) musa- 
shiana (Yokoyama), O. (Crassostrea) gigas (Thumberg), Cardita sp., OVenericardia (Megacardita) panda (YoKoYAMA), V. (M.) granulicostata Nomura, Thyasira (Conchocele) cfr. disjuncta (Gould), Lucinoma annulata (REEve), OFulvia mutica (REEve), Callista (Callista) chinensis (HoLTEN), Mercenaria stimpsoni (Gould), Glementia (Clementia) papyracea GRAY, Dosinia (Phacosoma) troscheli (LISCHKE), D. (Dosinorbis) bilunulata (GRAY), O Paphia (Paphia) takanabensis Shuto, Placamen tiara (DILWYN), Coelomactra antiquata (SPENGER), Solecurtes dunkeri KurodA, Cuspidaria (Cuspidaria) hindsiana (A. ADAMs), Lischkeia n. sp., Calliostoma (Tristichotrochus) shinagawaense cipangoanum (Yoкоуама), Suchium (Suchium) suchiense subsuchiense MAKIYAMA, Bittium sp., Polinices (Mammilaria) sagamiensis (PILSBRY), Tonna canaliculata (Linné), Ocenebra adunka (Sowerdy), Murex (Murex) cfr. djarianensis MARTiN, Rapana aff. bulbosa (ReEve), Babylonia elata (Yokoyama), Plicifusus sp., Granulifusus dualis (YoкоYамA), Fusinus simplex Sмгтн, Hemifusus sp., Oliva (Oliva) mustellina LAMARCK, Ancilla (Baryspira) albocallosa okawai (YoKoYAMA), A. (B.) oyamai SHuto, Fulgoraria (Fulgoraria) cancellata KuRodA and HABE, Lyria mizuhonica mizuhonica MAKIYAMA, Spirotropis subdeclivis (YoKoYAMA), Leucosyrinx coreanica (A. ADAMS), Turricula (Orthosurcula) australis (RoIssy), Bathytoma luedorfi Lischke, Philine scalpta A. Adams.

II-The stratigraphic positions of the fossil localities

The above mentioned fossil-localities are not uniformly scattered throughout the Miyazaki group, but differentially concentrated in certain facies and at certain stratigraphic horizons as indicated in the geological map. The lower part of the group is rich in molluscan fossils throughout entire areas and facies, but in the middle and the upper parts the majority of the fossiliferous beds is found only in the Tsuma facies, and in other facies only a few localities with meager assemblages are known.

These localities may be grouped into six horizons* in rather natural way and the six horizons are named here for convenience the first to sixth fossil horizon in ascending order.

The first fossil horizon has an unnegligible time range and shows a general tendency that the more north the geographic position, the higher the stratigraphic position caused by abutting, although the fossil beds are rather continuous one another. Thus the stratigraphic position of the horizon at the northern extremity is slightly but apparently different from those at the southern localities. This is the reason to subdivide this horizon into first- $\alpha$ (south) and first- $\beta$ (north) horizons. The first- contains Akatani, Yanaze, Kurawa, Waritsuke, Mokudo, Kawarada, Kano, Hamako, Kanekakematsu, Oyamada, Yusunokihashi, Uchinohaye, Horiguchi, Haigano, Kariyabaru, Uyeharu, Hôkôbô, Obira, Matsugano, northeast

* Strictly speaking, the term horizon should represent the time plane and not the strata of any time range. For the requirements of present stratigraphy, however, the term horizon can be used so as to cover a limited thickness of strata which range for so short time interval that the geological contemporaneity can be represented by that part. 
of Kagamisu pass, Kagamisu pass, Kaichigô, Maruno, Takeuchi, Inohaye, Uchinota, Kiyama, Kaburake, and Setare; and the first- $\beta$ contains Kakoi, Azukino, Kanekura, Kushiki, Jinkundani, Nakabyu, and Kugino. The second fossil horizon includes the localities Yamji, Nagano, Morinaga, Tsubone, and Takajô. Tonogôri, Yamasumi, and a locality $600 \mathrm{~m}$. west of Nishinobyu belong to the third one. The fourth includes Hagenoshita, Nishinobyu, Idenouye, and Kônji. The fifth contains Nihonmatsu and Iyedoko; and the sixth covers Tôriyama, Iwawaki, and Kizukume.

III-Physico-chemical aspects of the fossil beds of the first horizon

Palaeoecology concerns with the mutual relation between the ancient organisms and their environments, both organic and inorganic. The representatives of the physico-chemical factors of the ancient environment are nothing but the sedimentary rocks deposited in that environment, although the whole environmental factors are not always preserved in the rocks. Some parts of the factors fail to leave their records in the rocks. However others of them apparently affect the sedimentation in any way and accordingly these ancient factors may be evaluated indirectly with regard to the respective speciality through the observation or measurements of the particular physico-chemical features of the rocks. Furthermore a few but important factors can be evaluated directly through the measurements of the character in themselves. Therefore the sedimentary rocks make up themselves the best and sole criteria, though not perfect, for the consideration of the ancient environment. In this section the physico-chemical aspects of the fossil beds are described for the basis of the comprehension of the environmental conditions.

As above mentioned the fossiliferous beds are most extensively developed in the first horizon irrespective of the difference in the major sedimentary facies.

The fossil beds of the first horizon occupy mainly the silty fine sandstone of the upper half of the Boroishi and Tano member and the irregular alternation of siltstones and sandstones of the upper part of the Kawabaru member. Their thickness is varying from 30 to $300 \mathrm{~m}$. by locations. The lower part of the southern half is slightly lower stratigraphically than the northern half because of abutting phenomena as indicated in text-figs. 2, 3, 4, and 5 and plate 3 . The southern half south of Yatsushiro-mura is named, for convenience, the first- $\alpha$ and the northern part first- $\beta$.

The fossil bed of the first- $\alpha$ horizon is highly calcareous, grey, and silty sandstone of about ten $\mathrm{m}$. at Nangô and grey fine sandstone of a few tens meters at Nichinan. They are indistinctly stratified by a few meters and become coarse dipwards in a few hundreds meters. At both localities they are underlain by the stratified, thick, and medium sandstones and overlain by the alternation of sandstones and siltstones, which is in turn covered by the thick, grey, hard, monotonous, and coarse siltstone. The greater parts of the pelecypod fossils are the forms characteristic to the coastal waters and buried with conjoined values. At Uchinota, a little north of Kiyama, the assemblage chiefly consists of the 
openwater forms and many pelecypod valves are separated and buried in the sediment with the convex sides upwards. However they are not subjected to distinct erosion. This area may represent the margin or inlet of the protected mud flat. Hence a quiet and shallow mud flat with the off-shore sand bar and with the northern inlet may be assumed in the area from Nangô to Uchinota. At Kitagô mura the lithology of the upper part of the Boroishi member rather resembles that of the preceding area with the features that the sandstones are graded finer and finer upwards and become irregualr alternation of fine sandstones and siltstones. The molluscan fossils occur in parts of these calcareous grey fine sandstones with meager stratification.

At Boroishi there develops the thick alternation sequence of highly calcareous, coarse to medium sandstones and conglomerates above the basal conglomerates. The fine to medium sandstones of this part yield some molluscan fossils of warm open water elements. This part is overlain by thick barren sequence of conglomerates and sandstones, which is in turn covered by the fossiliferous medium to fine sandstones intercalated with some conglomerates. The fossils of this part is characterized by the exclusive predominance of species of Operculina, with rare, fragmental, or abraded occurrence of the subordinate species. Above this sequence the grey monotonous silty sandstone of the first- $a$ horizon is developed and yields much molluscan fossils, which show the evidence of the reworking after damage before burial. These facts suggest that the area, even in the time favourable for benthos lives, was under the moderate influence of the current or wave action of the open water.

At Tano the fossil beds of the first-a horizon are poorly sorted, represented largely by grey silty fine sandstones containing some patches of coarse to medium sand, and stratified only partially and irregularly. The remains of the benthos are assumed to have been subjected to the wave actinn and found sporadically but uniformly throughout these beds except in the coarse-grained part.

Northwards the lithofacies changes abruptly coarser and at Uchinohaye and Horiguchi the fossil beds are composed of the calcareous fine sandstones with coarse sand mixture. Further north at Yusunokihashi and Kanekakematsu they are very hard with much more content of coarse and medium sands than the rocks at Horiguchi and show the tendency for lensing reflecting the buried ridge geomorphology.

In the Aya area the main part of the fossil beds are composed of the monotonous, grey, and massive or scarcely stratified silty sandstones, yielding Paphia, Crassatellites, Ventricoloidea etc. and grade upwards into the grey, hard, and sandy siltstone with Acila. The total thickness of the fossil beds is about $200 \mathrm{~m}$. In this area the sediments represent the mudflat deposition in the open embayment like the Tano area.

North of Aya the fossiliferous beds branch into two parts with the intercalation of alternating, thick, and irregular beds of barren sandstones and siltstones between the two fossiliferous beds. The lower fossil beds are grey, massive, and sandy siltstone and the upper ones are composed of the alternation of fine 
sandstones and siltstones.

Furthernorth the lower fossil beds become coarser, gradually decrease in fossil contents, and finally disappear north of Fukuôji of Sanzai-mura. The upper fossil beds, representing the first- $\beta$ horizon here, are underlain by the coarse alternating sequence of coglomerates and coarse to medium sandstones. This alternation part is generally characterized by the round-pebble conglomerates and thick, massive, and unconsolidated fine sand lenses rich in plant drifts. The first- $\beta$ horizon itself is composed of the lithologically different two parts, of which the lower part consists of the alternation of the sandy siltstones and silty sandstones with irregular bedding of a few meters thick and is occasionally intercalated by thin sandstones. The upper part is chiefly silty sands but partly replaced by the alternation with indistinct bedding. Fossils occur both in the lower and the upper parts, and the assemblages from the lower part are dominated by Paphia, Acila, Glycymeris, Keenaea etc. and those from the upper by deeper forms such as Acila, Limopsis, Cuspidaria etc. These fossil beds occupy an outside belt of a large delta projecting into a kettle basin.

Summarizing the above mentioned evidences, the first horizon is differentiated lithologically into six parts; monotonous and massive sequence of Tano and Aya embayment areas; irregular alternation of northern area (Tsuma area) representing the marginal belt of a kettle basin; coarser and calcareous representatives of Yusunokihashi and Boroishi ridges; and the highly calcareous, scarcely sorted silty sandstones of the protected mud flat of Nangô-Nichinan area. This areal lithologic differentiation of the fossil beds in various facies is more readily understood through the examination of the total lithologic characters of the sequences underlying and overlying these fossil beds. In other words the differentiated parts in the fossil beds roughly correspond to the mega facies in the Miyazaki group.

To find out the minor differences in the contents of carbonates and nutrient organic matter in the fossil beds I have attempted the differential thermal analysis (D-T-A.) of about fourty samples of the sediments from various localities. The results indicate that the D-T-A. curves of the silty sandstones have some apparent relation to the stratigraphic position of the examined samples and to the distance from the shore margin. A very distinctly recognized tendency is that the lower or more marginal in the stratigraphic position of the localities, the more carbonate is included in the samples. D-T-A. also reveals the evident difference in certain other components of the rock samples by area. The fossil beds in the northern half of the Aoshima facies are most deficient in the organic matter and in the carbonate content and thus clearly distinguished from those in other areas. D-T-A. curves of the rock samples from the fossil beds of the first horizon in the Tano, Aya, Tsuma, and the southern half of the Aoshima facies do not differ essentially from one another, except that the curves of the Tsuma materials show an apparent peak caused by pyrite.

It may be presumably concluded on the basis of the D-T-A. curves that (1) the fossil beds of the first- $a$ horizon of the northern half of the Aoshima facies 


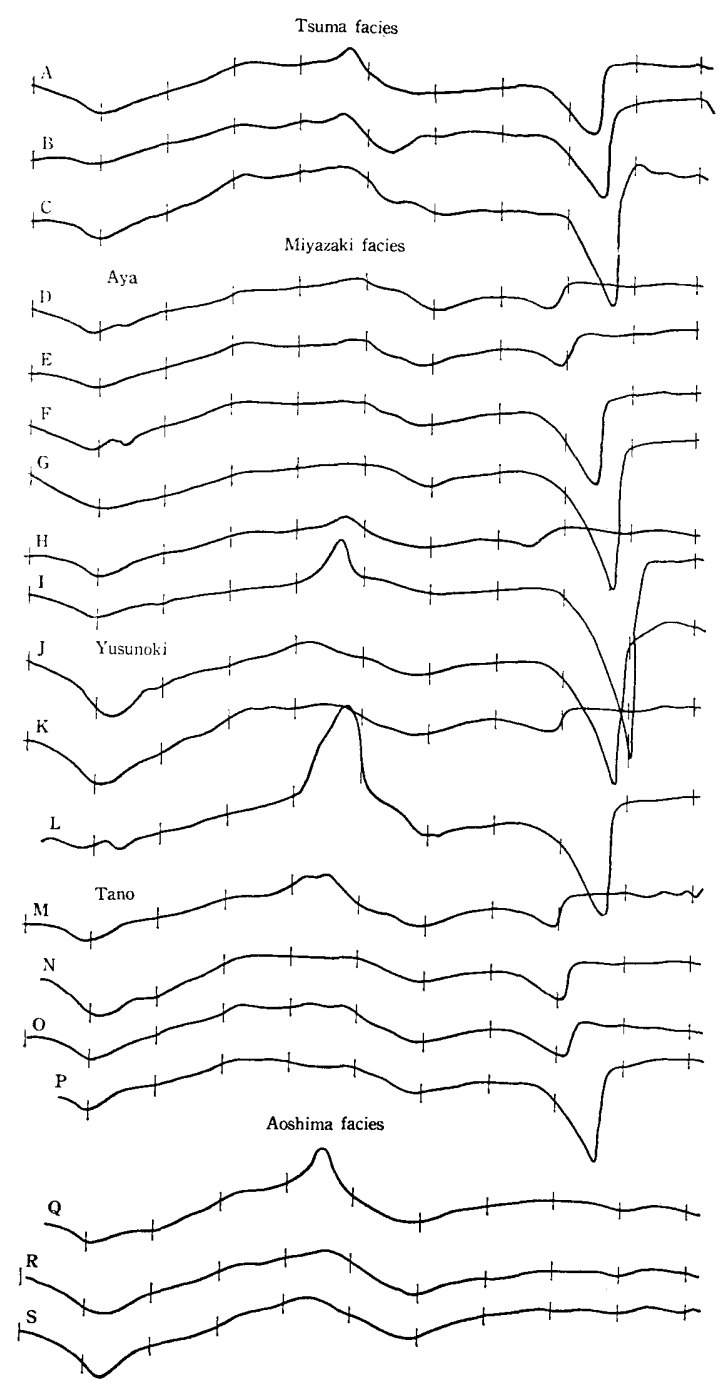

Text-fig. 6. D-T-A curves of the selected specimens of silty sandstones from the lower part of the Miyazaki group.

A....Nagano ; B....Yamaji ; C...Kakoi ; D....Kano ; E....Akatani-a ; F....Akatani-b; G....Yanaze; H....Kurawa-a ; I...Kurawa-b ; J... Oyamada; K...Uchinohaye ; L...Horiguchi ; M....Haigano ; N... Obira ; O....Hôkôbô ; P....Kariyabaru ; Q.... Kaichigô ; R.... Inohaye ; S....Uchinota.

characterized by the paucity of the organic matter and carbonates may represent the deposition in the open sea under the intense action of the water; (2) those of the southern half of the Aoshima facies, of the Tano and Aya areas of the Miyazaki facies, and of the Tsuma facies rich in carbonates and organic matter are considered to be the sediments in the quiet environments more or less protected from the direct influence of the open water; (3) the pyrite-peak of the Tsuma 
material means that the area was under more restricted condition than other facies.

It is noticeable that the result of this assumption, though rough and bold, is well harmonizing with the conclusion from the analysis of the sedimentaryfacies of the whole Miyazaki group and especially from the analysis of the biofacies of the first horizon, which is given below.

IV-Biological aspects of the fossil beds of the first horizon

As indicated in the description of the fossil localities and their fossil assemblages, some localities really yield the allochthonous elements derived from shallower or deeper depth after death. However these cases are rather exceptional and the allochthonous elements occupy only a very small fraction of each assemblage, if exist. Accordingly the greater parts are of the autochthonous or at least semiautochthonous in origin. This is verified by the following facts; namely, the occurrence of the fossils is sporadic in the matrix, the conjoined valves are frequently preserved in situ, abrasion effect is slight or negligible even on the odd valves of pelecypods, and the sand pipes accompanied with the shells are frequently preserved.

The water temperature, salinity, and depth of the basin bottom at the time of sedimentation of certain beds may be assumed rather acculately through the analysis of the autochthonous assemblages of the benthonic forms in these beds. As well known the water temperature affects the aquatic organic lives acting as limiting factor, and each species realizes its life in the distinct range of tolerance of temperature. However it is difficult and not practical to examine the exact thermal tolerance of numberless species. Within the Japano-Philippine faunal province the thermal range of the species which dwell in the surface water is approximately representd by the latitudinal range of the habitat of each species, since the present thermal range of the water at any latitude has been known. The vertical range of the surface water is now about $600 \mathrm{~m}$. in the Kuroshio water near the north extremity, Sagami bay. Thus we can roughly estimate the thermal tolerance of the species on the basis of its latitudinal range of distribution. In the case that an autochthonous assemblage of neritic benthos is given, we prot the known range of each constituent of the assemblage on the diagram as a bar (text-fig. 7) and sum up the number of the species at each latitude (text-fig. 8). The conseqent cumulative curve indicates the multiple latitudinal range of the assemblage, i.e. the possible thermal range of the assemblage on the whole. In the case of the fossil material, the older the geologic age, the less the percentage of the living species, and accordingly the more erroneous the assumption concerning with the thermal range of the assemblage. To decrease the possible error the ranges of the related living species are used in place of the extinct forms. The diagrams in text-fig. 8 were prepared generally according to IDA's method (IDA, 1956, pp. 15-20).

The fossil assemblages from the first- $\alpha$ and first- $\beta$ horizon contain the living forms by about twenty-nine and thirty-four percent of total number of the 
identified species respectively. As indicated in the diagrams the cumulative curves of the latitudinal range of only the living forms in each assemblage of the first horizon do not differ essentially from those of the recent faunas of Kii and Enshî. While that curve of the total species containing living and extinct agrees with that of the molluscan fauna of north Formosa, where the temperature of the surface sea water ranges between $20^{\circ} \mathrm{C}$ in winter and $28^{\circ} \mathrm{C}$ in summer. In other words, the molluscan assemblages of the first horizon, which contain much extinct warm water forms, might have dwelled within the temperature range from about $20^{\circ} \mathrm{C}$ to $28^{\circ} \mathrm{C}$.

The depth of the habitat of the fossil assemblage may be assumed through the average depth range of all the constituent species which is calculated through the examination of the depth ranges of living forms and living allies of the extinct forms. Of course the careful consideration is needed in dealing with the materials from different geomorphologic environments such as shelf or kettle basin, for the apparent tendency has been recognized that the same species ranges downwards on deeper bottom of the kettle basin than on the normal shelf (for example: Tsuchi, 1958, 69-76).

The fossil beds of the first horizon are further divided stratigraphicaily into

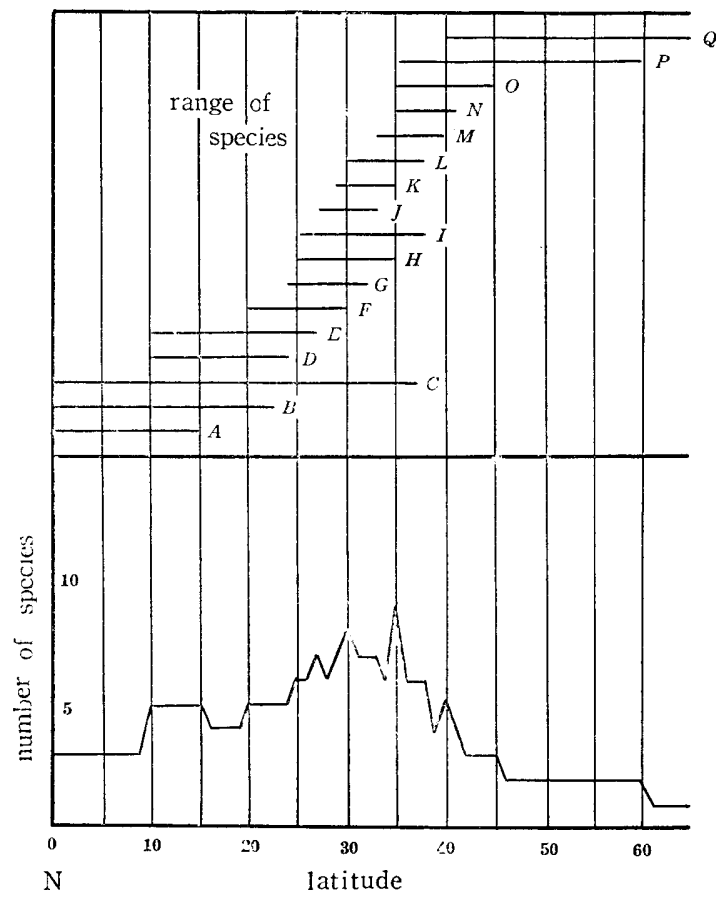

Text-fig. 7. Graphic method showing the character of the faunas. In the upper figure the bars A, B, C, etc. indicate the latitudinal range of individual species. The lower figure, in which the number of the species at each latitude is given, indicates the approximate faunal character with regard to the thermal condition of the sea waters. 

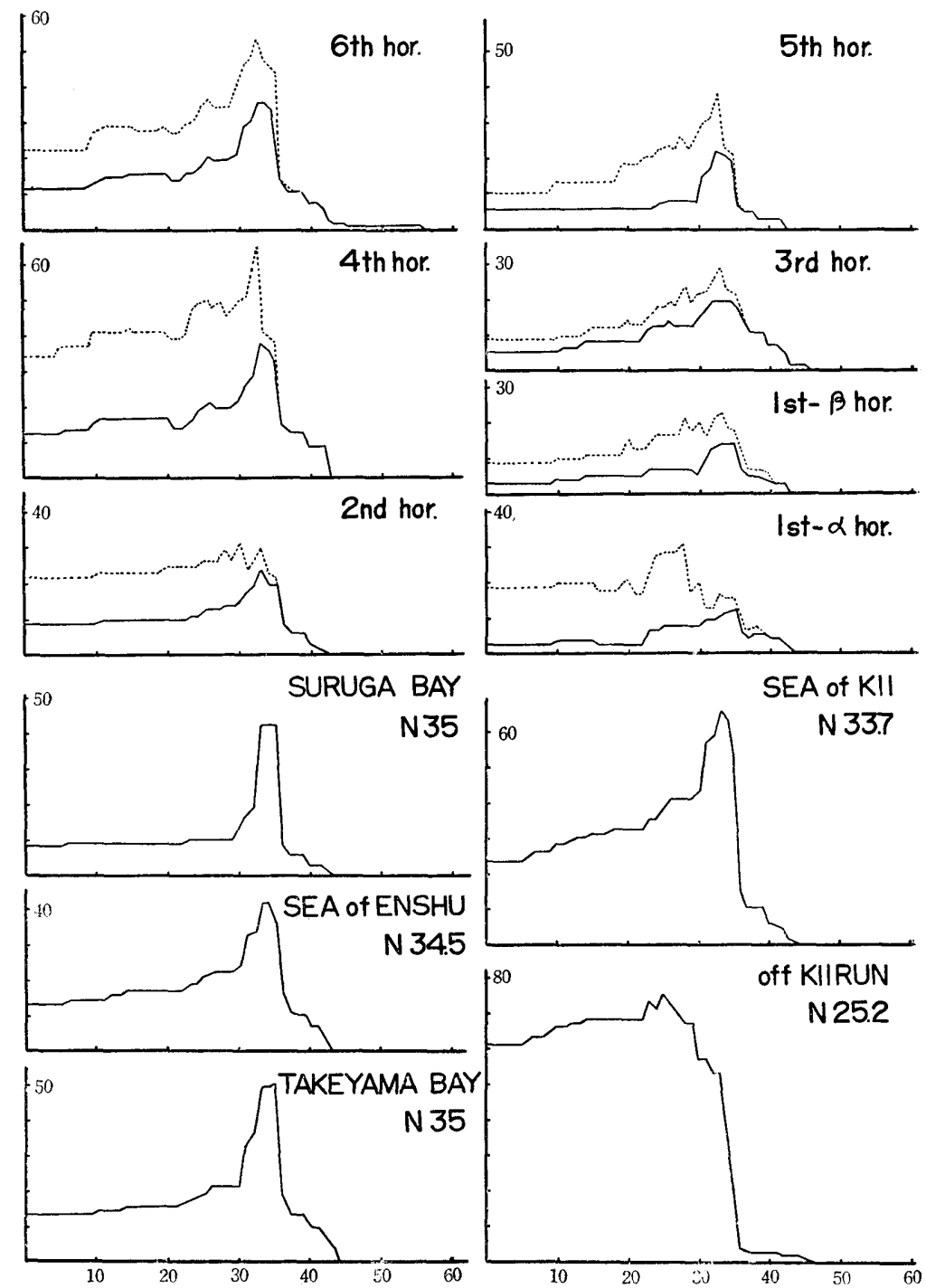

Text-fig. 8. Graphic presentation of the thermal range of the faunas in the term of the latitude.

Note the resemblance between the graphs based on the assemblages of the upper horizons of the Miyazaki group and the living faunas off Japan, and also between those of the lower assemblages of the group and the present fauna off Kiirun, Formosa.

The solid lines are based on the number of the living species and the dottedlines are based on those of the living allies of the extinct species.

three parts, the lower $\alpha_{1}$, the main middle $a_{2}$ and $\beta_{2}$, and the upper $\beta_{33}$. The assemblages of the lower part (first- $\alpha_{1}$ ) including Kaburake, Setare, Kiyama, Mt. Boroishi, Uyeharu-b, and Mokudô assemblages consist of the elements dwelling on the euneritic to mesomeritic $\left(\mathrm{N}_{1}-\mathrm{N}_{2}\right)$ fascia. Those of the middle main part 
(first- $a_{2}$ and $\beta_{2}$ ), including the assemblages of Inohaye, Kaichigô, Maruno, Kagamisu pass, Hôkôbô, Kariyabaru, Haigano, Akatani, Waritsuke, Kugino, Kakoi, Azukino-a etc. are composed of the mesoneritic to subneritic $\left(\mathrm{N}_{2}-\mathrm{N}_{3}\right)$ elements. The upper part (first- $\beta_{3}$ ) is characterized by the deeper water (bathyneritic to to bathyal, i.e. $\mathrm{N}_{4}$-B) elements at Kawarada, Takeuchi, Kanekura, Nagano, Azukino-b, Takajô, etc.

Either of these parts are laterally seggregated into a few biofacies with particular structure of the organic community. The main part is described here at first.

Paphia (Paphia) exilis exilis SHUto, P. (P.) exilis abbreviata SHUTo, Joannisiella cumingii (HANLEY), Venus (Ventricoidea) foveolata miyazakiensis (SHuto), and Clementia papyracea (GRAY) make the off shore faunule of Aya embayment distinctive. Pitar (Pitarina) dohrni (RöMER), P. (P.) semeliformis Shuto, Paphia (Paphia) hirabayashii tanoensis Shuto, P. (P.) exilis takaokaensis SHuto, and Turrid gastropods are the subordinate elements of this assemblage. In other words this assemblage is almost purely composed of the pelecypod species and contain only few gastropods. The similar assemblages occurs at a few localities in the upper Oligocene Ashiya group, which consist chiefly of Dosinia (Phacosoma), Pitar, Callista, and Lucinoma. At present time the analogous faunas are often developed on the muddy bottom of the partly protected waters. These pelecypods, the detritus- and plankton-eaters, form the part of the lower level of the food chain and accordingly the density of their total population depends mainly on the supply of the food material and on the quantity of the carnivora if the other conditions are equal. Since there is no or least records of the carnivora, the ecological pyramid of that area might have extremely small apex in comparison with the large middle layer. In this case abnormal paucity of the gastropods may be caused by the limiting factor, the muddy bottom, rather than the food.

The above mentioned species do not always occur frequently and uniformly throughout the off shore area, but the structure of the molluscan assemblage generally differs in details by place. A few pervading species are found together at almost equal frequency where the population density is not high, but the localities with dense population are characterized by the dominance of one or two species. For example the dense assemblage is dominated by Crassatellites (Crassatellites) tenuiliratus SHUTo at Kano, and by Paphia (Paphia) exilis exilis SHUTo at Akatani and Kano.

The near shore assemblage of Aya area is characterized by the dominance of Amussiopecten iitomiensis (OTUKa), Paphia (Paphia) exilis takaokaensis SHUTo, and Siphonalia praedeclivis ITOIGAWA and also contains Ventricoloidea foveolata miyazakiensis (SHuto), Solecurtes divaricatus LISCHKE, and Balanus as the subordinate elements.

The assemblage of the middle fossil part of the Tano area, where the lithology is rather muddy like as that of the Aya area, is featured by the predominance of Clementia payracea (GRAY), Paphia (Paphia) exilis takaokaensis SHUto, Joan- 
nisiella cumingii (HANLEY), Siphonalia praedeclivis ITOIGAWA, Carcinoplax antiqua (RISTORI), and Schizaster hyugaensis MoRISHITA at the off shore area of the embayment. At the particularly muddy localities of the center of the embayment it is almost purely composed of Clementia and Carcinoplax with high density. In the marginal coarser sediments of shallower depth Amussiopecten iitomiensis (Otuka), Paphia exilis takaokaensis Shuto, Murex, Siphonalia, Eudolium, Conus etc. occur abundantly.

The assemblage of Yusunoki peninsula, especially of northeast side of it, is characterized by fully grown specimens of Aussiopecten iitomiensis (OTUKA), Paphia exilis takaokaensis SHUTO, and Brachiopods, which comprise the "PectenBrachiopod" community (OYAMA, 1954-b, 97-99), indicating the strong water action at the time of sedimentation.

The Boroishi-Kitagô assemblage consists dominantly of Paphia exilis takaokaensis SHUTо, Amussiopecten iitomiensis (OTUKA), Solecurtes divaricatus (LischKe), Eudolium, and Siphonalia, the greater parts of which more or less suffered the abrasion by water action. The Nichinan-Nangô assemblage is specialized by Anadara daitokudoensis MakiYama, A. rhombea (Born), A. valentula (Yokoyama), Dosinia etc. at the south and Paphia, Anadara, Vasticardium etc. at the north. These assemblages as well as the lithology indicate the protected environment against open water with a inlet at the north.

The Tsuma assemblage is characterized as a whole by the frequent occurrence of Acila submirabilis MaKiYAMA, Saccella confusa kongiensis (OTUKa), Glycymeris rotunda Duker, G. formosana Yokoyama, Dosinia nomurai Otuka, Paphia hirabayashii koyuensis SHUTO, Joannisiella cumingii (HANLEY), Keenaea samarangae (MAKIYAMA), Glossaulax reiniana (DUNKER) subsp., Nassaria magnifica LISCHKE n. subsp., and Spirotropis subdeclivis (YoKOYAMA) n. subsp. While the assemblage at each locality in this area is specialized by the subordinate elements such as Crassatellites (Crassatellites) tsumaensis SHuTo, Venericardia (Megacardita) megacostata SHUTo, and Suchium at Yamaji-b, Vepricardium kyushuense Shuto, Mercenaria chitaniana (YokoYama), and Granulifusus koyuanus SHuto at Azukino-a, Lischkeia alwinae (LISCHKE), Galeodea n. sp., Murex, and Vepricardium at Kakoi, and Callista chinensis (HoLten) at Nakabyu.

The assemblages of the upper part of the fossil beds are divided into two facies: Those of the Aya and Tano area are led by Acila submirabilis Makryama and those of Tsuma area north of Kugino, Aya machi are characterized by Acila submirabilis MAKIYAMA and Limopsis obliqua A. AdAMs. At the BoroishiNichinan area this part contains practically no mollusks. While immediate north of the Boroishi ridge the typical "Lucinoma-Conchocele" community with the subordinate elements such as Clinopegma unicum (PILSBRY) and Palliolum peckhami (GABB) is developed which indicates relatively deep water condition.

In brief the main fossil part (first- $a_{2}$ and $\beta_{2}$ ) is clearly differentiated into the following biofacies; they are Paphia-Ventricoloidea-Crassatellites assemblage of Aya, Paphia-Amussiopecten-Brachiopoda assemblage of Yusunoki, ClementiaPaphia-Carcinoplax-Schizaster assemblage of Tano, Amussiopecten-Paphia assem- 
blage of Boroishi-Kitagô area, Anadara-Dosinia assemblage of Nangô, and Joannisiella-Keenaea-Glycymeris-Glossaulax assemblage of Tsuma. The former four assemblages may be grouped as Amussiopecten-Paphia assemblage. Each assemblage represents the particular influence of the oceanic or coastal water and of the bottom sediments.

Then it should be examined whether any environmental fluctuation or development is indicated in the vertical succession or not. In Aya area the basal coarse conglomerate is overlain by the granule conglomerate which yields Notostrea sp., Glycymeris formosana Yokoyama, Amussiopecten iitomiensis (OTUka), Trachycaridum cfr. shiobarense (Yokoyama), and Paphia hirabayashii tanoensis SHUTo. All the specimens except for Notostrea sp. are more or less abraded and considered to have been derived from deeper depth after damage. The overlying coarse sandstone yields abundantly Operculina complanata japonica HANZAWA, which indicates shallow warm open water condition. This bed is succeeded by the highly calcareous alternation of barren coarse to medium sandstones in a few tens meters cycles. Upon this lies the poorly sorted sandstone of Waritsuke, which is characterized by such purely open water elements as Veletuceta reevei (MAYER), Amussiopecten, Pycnodonta, Vasticardium, Dosinia (Bonartemis), Solecurtes, and Grammatomya. Overlying thick sequence consisting of the grey fine sandstones in irregular alternation is deficient in fossils. In higher horizon at Yanaze the beds yields abundant fossils consisting of only a few elements such as Amussiopecten, Ventricoloidea, Paphia exilis exilis SHUto, and $P$. exilis takaokaensis SHUTo and is succeeded by the rarely stratified sandstone, which sporadically bears the fully grown specimens of Amussiopecten and subordinately Paphia exilis takaokaensis SHUto and Siphonalia. These indicate that the area was under the strong influence of the open water in that time. However thereafter the influence of the shore water gradually increased. Thick massive silty sandstone sequence is a reflection of partially protected calm environment on the gently sinking basement and yields such coastal water elements as Joannisiella cumingii HANLEY, Clementia papyracea GRAY, and Glossaulax besides the open water elements. Thereafter the deposition was led by the sinking and this resulted the finer sediments with deeper faunule, consisting chiefly of Acila, Nuculana, and Cuspidaria. In other words in Aya area an apparent and gradual environmental change proceeded from purely open water condition to the condition under the moderate influence of the coastal water and from shallower $\left(\mathrm{N}_{1}\right)$ to deeper $\left(\mathrm{N}_{3}, \mathrm{~N}_{4}\right)$.

In Tano area below the Operculina horizon a thick sequence of barren sandstones and conglomerates is developed. The rock of the Operculina bed itself is similar to that of the Aya area and composed of calcareous coarse washed sandstone. It is overlain by the incompletely sorted greenish grey, medium to fine sandstone which yields the minute forms of Solen and Laevicardium. These genera are the good indicators of the bay water. These serial evidences suggest that the original coast, washed by the open oceanic water, was gradually filled up by poorly sorted material, and that the shallow muddy sand flat was formed so 
extensively that the invasion of the oceanic water was more or less prevented. As the transgression proceeded the flat became slightly deeper and more muddy and on this quiet environment the large group of benthonic organisms flourished with the marginal variable assemblages and the central rather simple one as. described in the preceding paragraphs.

In both area the ancient environments of first-a horizon gradually and apparently changed from shallow oceanic condition to deeper water condition under the influence of the coastal water.

The Operculina sandstone does not extend northwards beyond Hokkedake in Yatsushiro mura, though the sandstones of similar grain size but less calcareous. are developed equally further north. Amussiopecten-Paphia exilis beds also disappear north of the northern extremity of Yatsushiro mura. The distribution of these forms must have been ruled by the environmental conditions. The deltaic sediments are formed occupying the lower part of the Tsuma facies in a large scale at Mino mura and its adjacents. The river which charged the deltaic sediments must have drainaged vast volume of fresh water. The marine organisms must have been subjected to the influence of the drainage fresh water in the leeside area (south) to the mouth of the river beyond the outer extremity of the delta itself. This may be the sole reason why the fossil beds, extending from south, disappear abruptly north of Yatsushiro mura and other fossil beds exist outside and over the deltaic body.

Comparing with the assemblages of various localities south of Aya, those of the Tsuma area are characterized by the absence of the purely open sea elements. such as Schizaster, Carcinoplax, Amussiopecten, and Paphia exilis takaokaensis SHUTo and by the presence of various genera of Naticids, Paphia hirabayashii koyuensis Shuto, and Keenaea. Furthermore a bay water element, Joannisiella cumingii (HANLEY) attains full growth in this area and Dosinia (Phacosoma) nomurai OTUKA takes the place of the open water form, D. (Bonartemis) suketoensis (OTUKA).

That is to say Tsuma area must have been influenced more severely by the coastal water than the south areas.

V-Biological features of the fossil beds of the second to the sixth horizons

At the second and the upper horizons the fertile fossil beds scarcely developed in the Miyazaki and Aoshima facies where the thin-cycled alternations are extensively developed, and the important fossil beds are found exclusively in the Tsuma facies.

The cumulative curve of the latitudinal range of the constituents of the fossil assemblages of the first horizon resembles those of the recent assemblages: of the tropic waters and those of the third to sixth horizons agree to that of the subtropic Kii fauna. That of the second horizon is intermediate between the above two types. Almost all the fossil assemblages of the six horizons are considered to be of mesoneritic to subneritic fascia $\left(\mathrm{N}_{2}\right.$ to $\left.\mathrm{N}_{3}\right)$. These facts indicate that the temperature of the surface water gradually lowered down approximately 
from $20-28^{\circ} \mathrm{c}$. of the tropic condition to $14-22^{\circ} \mathrm{c}$. of the off shore waters of Kii in the lapse of time from the first to the third horizon and thereafter the thermal condition did not change essentially.

In the Tsuma area the lithologic character of the second horizon shows minor change laterally and vertically reflecting the variability in the environmental conditions. Consequently numerous small bodies of sandstones and silty sandstones are intercalated in the stratified silty sequence and the fossil beds are not extensive but small and rare. For example, Ancilla (Turrancilla), Limopsis, and Fulgoraria (Psephaea) community in the greenish grey siltstone at Kanekura; Parvamussium (Parvamussium), Cuspidaria (Cuspidaria), and Fulgoraria (Psephaea) community in the grey sandy siltstone at Nagano; Acila, Merisca, Ancilla (Baryspira), Thatcheria, and Inquisitor cummunity in the silty sandstone at Takajô; Lima (Acesta), Limopsis, Cuspidaria (Cuspidaria), Cuspidaria (Cardiomya), and Spirotropis cummunity in the grey siltstone south of Yamaji etc. All the above mentioned localities are placed in a small area closely adjoining one another.

In the Aya area the situation is quite difference. A very remarkable shell bank is developed at Morinaga for the length of $1500 \mathrm{~m}$. along the strike trend. It is largely composed of the valves of Amussiopecten iitomiensis (OTUKA) and accompanied by Paphia exilis takaokaensis SHUTo in a small fraction. It means that the favourable habitat for the life of the cited species existed on or near the shell bank.

In conclusion there were two contrasting environments, the purely open water condition and the one affected by the coastal water, at the time of the second horizon.

It is questionable from the ecological point of view that Clinocardium is found in the warm water assemblages of the lower part of the Miyazaki group and also of the middle Miocene beds of our country from Kyushu to Hokkaido as well as among other temperate water faunas. This genus is typically a neritic element of the cold waters as is well known from the Pliocene, Pleistocene, and Recent faunas in the north Pacific. The meaning of the fact has not been clarified yet.

The fossil assemblages of the third horizon in the Tsuma facies consists of two parts, the assemblages on the bottom of the muddy deep (Yamasumi and an area west of Nishinobyu) and those on the sandy bank (Tonogôri) which stretches into the muddy basin from south. The slumping sediments with contorted minor. structures and shallow elements of mollusks are found at a few localities around the bank sand bodies. The assemblages on the muddy bottom are typified by Perioploma, Pseudometis, Thyasira, Mangelia, and Operculina ammonoides (Gronovius) and those on the bank sand are characterized by the large and dense assemblages resembling the recent faunas on the coastal shelf sand off Tosa (=Kôchi), Kii (=Wakayama), and Enshû (=Shizuoka). Outside the Tsuma facies the sponge spicules, i.e. Sagarites chitanii MAKIYAMA, and Echinoderm. spines are found very abundantly from the grey mudstone in the alternations, 
but the mollusks are not known except for the rare and accidental occurrence of small pelecypods such as Acila, Palliolum, and Poromya. These facts indicate that the marine condition became open oceanic in both the Tsuma and the other areas, and the difference in the molluscan assemblages might have depended chiefly on the bottom material and the depth.

The assemblages of the fourth, fifth, and sixth horizons do not differ elementally from those of the third horizon. The faunules of the fourth horizon also include two types of the representatives, that is to say, the large assemblage of shallow depth at Hagenoshita and deeper one at Koonji. The former is characterized by Orectospira excelsa (YoKoYAMA), Uberella yokoyamai KURoDA and HaBe, Nassaria lischkei MakiYama, Siphonalia declivis YokoYama, Marginella novemprovincialis (YoKoYAMA), Turris kurodai MAKIYAMA, Leucosyrinx coreanica (ADAMs), Granulifusus dualis (YoKоYAMA), Venericardia (Megacardita) panda (YoKoYAMA), Glycymeris rotunda DUNKER etc. and the latter is featured by Acila divaricata (HINDS), Yoldia (Tepidoleda) naganumana (OTUKA), and Uberella yokoyamai KURODA and HABE. Hence they are compared with the assemblages of Tonogôri and Yamasumi of the third horizon respectively, but the difference in depth may not be so great. This noticeable tendency in the minor facies differentiation is also demonstrated at Hagenoshita where the difference in depth between the silty bottom and the sand bank became smaller at this horizon than it had been before. Two assemblages at Hagenoshita, one in the silt bed and the other in clean sand bed, are obviously distinctive from each other. However almost all the elements of the assemblage in the siltstone, except for Fulgoraria (Psephaea) kamakuraensis OTUKA, and Lyria mizuhonica koyuana (YoKoYAma), are found in that of the sand bed, which is very large and inclusive, containing many eurybenthic forms besides the typical sand dwellers such as Cryptopecten vesiculosus (DUNKER), Venericardia (Megacardita) panda (YoKoYAMA), V. (M.) granulicostata Nomura, Suchium, Siphonalia declivis YokoYama, Trigonostoma retc.

The fossil assemblages of the fifth horizon are quite similar to that of the fourth, and characterized by the frequent occurrence of Glycymeris rotunda Dunker, Orectospira excelsa (YoKoYAMA), Uberella yokoyamai KURoDA and HABE subsp., Nassaria lischkei MakiYama, Granulifusus dualis (YoKoYama), Marginella novemprovincialis (YoKOYAMA), Bathytoma striatotuberculata (YoKoYAMA), and Spirotropis subdeclivis (YoKoYAMA) in the sandstone, and Bathytoma, Granulifusus, and Spirotropis in the siltstone. It is remarkable that a pure Cucullaea granulosa JoNAs community is developed extensively in the fine sandstone at Idenouye in Kawaminami mura, though the ecological or zoogeographical meaning is not clear.

The sixth horizon is differentiated biologically into several local seggregations in spite of the lateral invariabilty of the lithology in the Tsuma facies. The large and dense assemblage at Tôriyama is characterized by Anadara castellata (YoKoYAma), Glycymeris rotunda DUnkeR. Amussiopecten praesignis (YoKoYAMa). Venericardia (Miegacardita) panda (Yoкоуама), Paphia takanabensis 
SHUTO and is naturally called Anadara castellata-Amussiopecten praesignis-Paphia takanabensis assemblage. The fossil beds at Heki and Iyedoko contain almost pure Volsella nitida community. The fossil group at Kizukume is also large and dense, which may be called Crassatellites takanabensis-Keenaea samarangaeLeucosyrinx coreanica community. At Iwawaki a very distinct shell reef consisting of the aberrant form of Lucinoma annulata (REEVE) is developed.

VI-Concluding remarks on the palaeoecology

Summarizing the palaeoecological remarks, the major changes of the ecological conditions through times are summarized here. Before describing the natural history a few general remarks ought to be given. From the palaeoecological research of the Miyazaki group, it can be stated that the limiting factors, i.e. inorganic environmental conditions display the clearer records than the structure of the biotic community. In other words the former seems to have played more fundamental roles in realizing the particular faunule at each locality than the mutual reaction of the organisms themselves had, though the chief subjects in the modern ecology are generally concerned with the social structure of the biotic community. In reality the palaeontologic materials are not yet thoroughly analysed for such a requirements. There are, however, some examples which may be explained from this side. For example a distinct social structure of the normal ecological pyramid is shown by the assemblage at Hagenoshita and also the differential communities caused by the purely biotic reaction in one and the same bed under the practically the same limiting factors are shown in the assemblages of the fossil bed of the sixth horizon.

Among the limitting factors the thermal conditions, salinity, depth of the waters, and the bottom material seem to be significant in ruling the marine animal community.

The temperature of the sea water lowered down gradually from tropic condition to subtropic in the lapse of time from the first to the third horizon and thereafter no essential change took place. From the bathymetric and hyaline stand point the major history is as follows: At first the oceanic water invaded on the land of low relief. Then, the sea became deeper rather rapidly on the general area as the transgression progressed, but reflecting the block movement or differential movement of the basement the area is differentiated laterally into deeper and shallower segments. Consequently, as regards the oceanic current, the north areas of the burried ridges were affected by the coastal water to which some bay water elements of mollusks were introduced. The occurrence of the bay water elements in such a shallow and open embayment is not inconsistent to the general tendency in the actual bays in the present time, since embayment fauna is really developed not only in the typical bay area, but also even in the small and open inlets protected only by the merged rock reef as illustrated at Norigoye of Sagami Gulf (OYAMA, 1954, p. 92) etc. The depth difference of shallower and deeper segments became smaller and smaller after the time of the third horizon until a rather flat muddy shelf was formed at the time of the sixth 
horizon. Keeping pace with this change the influence of the coastal water became weaker and weaker until the purely open sea condition was reached.

\section{Age of the faunas}

I-Analysis of the fossil assemblages of six horizons

The important fossil localities in the Miyazaki group are sorted into six horizons as mentioned in the section on the ecology. The molluscan fossils of each horizon are described from the chronological stand point.

(1) The first- $a$ fossil horizon (Akatani horizon)

Acila (Acila) submirabilis MaKIYAMA

Saccella confusa kongiensis OTUKA

Mio. - (Rec.)

Nuculana (Nuculana) cfr. pennula (YoKoYAMA)

Mio.

Yoldia (Tepidoleda) naganumana (OTUKA) n. subsp.

Mio.

Portlandia (Portlandella) japonica (AdAMS and ReEve)

Anadara (Scapharca) valentula (YoKOYAMA)

* (Plio. - Pleist.)

A. (S.) daitokudoensis MAKIYAMA

Mio. - Rec.

Mio.

A. (S.) rhombea (BORN)

Mio.

A. sp.

Cucullaea pamotanensis MARTIN

Plio. - Rec.

Glycymeric cfr. rotunda (DUNKER)

Low. Mio.

Amussiopecten iitomiensis (OTUKA)

Plio. - Rec.

Veletuceta reevei (MAYER)

Mio.

Palliolum (Delectopecten) peckhami (GABB)

Parvamussium (Parvamussium) kyushuense SHUTo

Rec.

Oligo. - Mio.

*

Ostrea (Pycnodonta) musashiana (YoKoYAMA)

O. (Nodostrea) sp.

Crassatellites (Crassatellites) tosanus NomurA

Plio. - Rec.

C. (C.) tenuiliratus tenuiliratus SHUTO

Plio.

C. (C.) tenuiliratus triangularis SHuto

Venericardia (Megacardita) megacostata SHuto

$V$. (M.) ferruginosa (A. ADAMS and REEVE)

Thyasira (Conchocele) nipponica YABE and NOMURA

Lucinoma acutilineata (CONRAD)

L. sp.

Joannisiella cumingii (HANLEY)

Vasticardium ogurai (OTUKA)

$V$. hyugaense SHUTо

Vepricardium kyushuense SHUTO

Trachycardium shiobarense (YoKoYAMA)

$\%$

$*$

$*$

Mio. - Rec.

Mio. - Plio.

Mio. - Plio.

Mio. - Rec.

Mio.

$*$

$*$

Mio.

* Asteriks in the column of the geologic range indicate the occurrence only in the Miyazaki group. 
Clinocardium shinjiense (YoKoYaMa) Mio.

Pitar (Pitarina) dohrni (RÖMER) Rec.

Laevicardium pigmae SHUTo *

P. (P.) cfr. sulfurea (PILSBRY) Plio. - Rec.

Venus (Ventricoloidea) foveolata miyazakiensis (SHUTo) *

$P$. (P.) semeliformis SHUTO

Dosinia (Bonartemis) suketoensis (ОTUKA)

D. (Phacosoma) sp.

Kaneharaia kaneharai (Yoкоуама)

Paphia (Paphia) hirabayashii tanoensis SHuto

$*$

$\approx$

$P$. (P.) exilis exilis SHUto

P. (P.) exilis takaokaensis SHuTo

$P$. (P.) exilis abbreviata SHuto

Mio.

Lutraria nenokamiensis KANNO

Solecurtes divaricatus LISCHKE

Grammatomya squamosa (LAMARCK)

Azorinus minutus (DUNKER)

Merisca n. sp.

Fabulina miyatensis (YoKOYAMA)

Thracia (Cyathodonta) dissimilis KURODA and HABE

Panope japonica REEVE

Cuspidaria (Cuspidaria) hindsiana (A. ADAMs)

Mio.

$*$ (Mio.)

兑

Mio. - Low. Plio.

$\ddot{*}$

Oligo.

Mio. - Rec.

Rec.

Rec.

$\%$

Plio. - Pleist.

Rec.

Mio. - Rec.

Rec.

C. (C.) n. sp. a

*

\%

C. (C.) n. sp. b

C. (Myonella) n. sp.

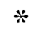

C. (M.) sp.

Pholadomya sp.

Calliostoma sp.

Tegula (Chlorostoma) yokoyamai NoMURA and HATAI

Turritella (Haustator) n. sp.

Architectonica (Stellaxis) n. sp.

Xenophora (Tugurium) dunkeri MARTIN

Epitonium (Boreoscala) nagamiensis OTUKA

Eudolium sp. b

Phalium (Bezoarium) japonica (REEVE)

Murex (Murex) n. sp.

Ancistrolepis (Clinopegma) unicus (PILSBRY)

Siphonalia praedeclivis ITOGAWA

Cantharus sp.

Fusinus 2 spp.

Hemifusus cfr. tuba (GMELIN)

Turris cfr. ugariensis MAKIYAMA

Parasyrinx sp.

Surculites yokoyamai OTUKA

Spirotropis subdeclivis (YoKoYama) n. subsp.

Mio.

$\%$

$\%$

Plio.

Plio.

Mio. - Rec.

$*$

Plio. - Rec.

关

Plio. - Rec.

Low. Plio.

Mio.

* 
Bathytoma n. sp.

Total number of the distinct species $(\mathrm{T}) \ldots \ldots \ldots \ldots \ldots \ldots \ldots \ldots \ldots \ldots$

Number of the inidentified species $(\mathrm{Sp}) \ldots \ldots \ldots \ldots \ldots \ldots \ldots \ldots \ldots \ldots$

Number of the species known only from the Miyazaki group (Msp) . . . 21

Number of the Oligocene element .................. 1

Number of the Oligocene to Miocene element $\ldots \ldots \ldots \ldots \ldots \ldots \ldots, 1$

1 st $-\alpha$ hor.

\begin{tabular}{|c|c|c|c|c|c|c|c|}
\hline \multirow[t]{9}{*}{ U. Oligo. } & L. Mio. & M.Mio. & U. Mio. & Plio. & Pleist. & Rec. & \\
\hline & & & & & & & 1 \\
\hline & & & & & & & 1 \\
\hline & & & & & & & 1 \\
\hline & & & & & & & 10 \\
\hline & & & & $=$ & & $\operatorname{sen} x=0$ & 6 \\
\hline & & & & & & & 4 \\
\hline & & & & & & & 6 \\
\hline & & & & & & $\operatorname{lax} x=0$ & 6 \\
\hline 2 & 4 & 20 & 20 & 21 & 14 & 18 & 43 \\
\hline
\end{tabular}

1 st $-\beta$ hor.

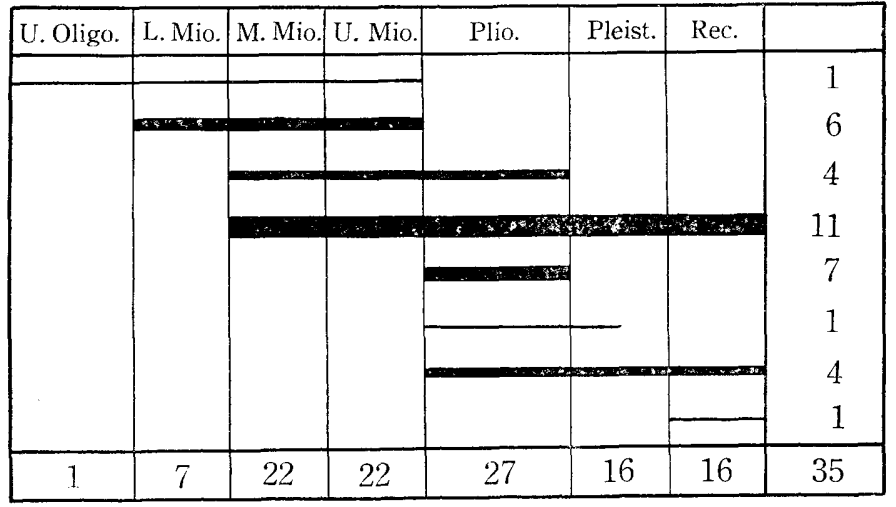

Text-fig. 9. Diagrammatic presentation of the composition of the molluscan assemblages of the first- $\alpha$ and first- $\beta$ horizon with regard to the known ranges of the species.

In each table the figures at the right-handed column indicate the number of the species of particular geologic range. The figure at the lower line of each column indicates the total number of the species at particular age. 
Number of the Miocene elements $\ldots \ldots \ldots \ldots \ldots \ldots \ldots \ldots \ldots \ldots \ldots$

Number of the Miocene to Pliocene elements $\ldots \ldots \ldots \ldots \ldots \ldots \ldots \ldots$

Number of the Miocene to Recent elements $\ldots \ldots \ldots \ldots \ldots \ldots \ldots \ldots 6$

Number of the Pliocene elements $\ldots \ldots \ldots \ldots \ldots \ldots \ldots \ldots \ldots \ldots \ldots$

Number of the Pliocene to Pleistocene elements .............. 2

Number of the Pliocene to Recent elements ............... 6

Number of the species known only from Recent $\ldots \ldots \ldots \ldots \ldots \ldots .6$

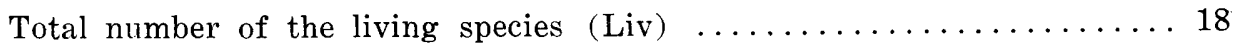

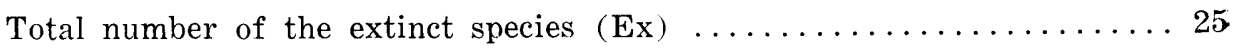

\begin{tabular}{|c|c|}
\hline $\mathrm{Liv} / \mathrm{T}$ & $23.4 \%$ \\
\hline $\mathrm{Ex} / \mathrm{T}$ & $32.5 \%$ \\
\hline $\mathrm{Liv} / \mathrm{T}-\mathrm{Sp} \ldots$ & $28.2 \%$ \\
\hline $\mathrm{Ex}+\mathrm{Msp} / \mathrm{T}-\mathrm{Sp}$ & $71.8 \%$ \\
\hline
\end{tabular}

(2) The first- $\beta$ fossil horizon (Kakoi horizon)

Acila (Acila) submirabilis MaKIYAMA

Saccella confusa kongiensis ОTUKA

Anadara (Scapharca) sp.

Cucullaea granulosa (JoNAs)

Glycymeris rotunda (DUNKER)

G. formosana (YoKoYAMA)

Limopsis obliqua A. ADAMS

Atrina (Servatrina) pectinata (LINNE)

Gloripallium miurensis (YoKоYамA)

Parvamussium (Parvamussium) kyushuense SHUTo

Palliolum (Delectopecten) peckhami (GABB)

Lima (Acesta) goliath ( SмITH)

Crassatellites (Crassatellites) tosanus NomurA

C. (Crassatina) oblongata uchidana (YokoYAMA)

Thyasira (Conchocele) nipponica YABE and NoMURA

Joannisiella cumingii (HANLEY)

Vepricardium kyushuense SHUTo

Nemocardium (Keenaea) samarangae (MAKIYAMA)

Pitar (Pitarina) pellucida (LAMARCK)

Callista (Callista) chinensis (HoLTEN)

Dosinia (Phacosoma) nomurai OTUKA

Mercenaria chitaniana (YoKoYAMA)

Clementia (Clementia) papyracea (GRAY)

Paphia (Paphia) hirabayashii koyuensis SHUTo

Cuspidaria (Cuspidaria) hindsiana (A. ADAMS)

C. (C.) n. sp. a

C. (Myonella) n. sp.

C. (Cardiomya) n. sp.

Lischkeia alwinae LISCHKE
Mio. - (Rec.)

Mio.

Mio. - Rec.

Plio. - Rec.

Plio.

Mio. - Rec.

Plio. - Rec.

Mio. - Plio.

*

Oligo. - Mio.

Mio. - Rec.

Low. Plio.

Plio.

Mio. - Plio.

Mio. - Rec.

$\%$

Mio. - Rec.

Rec.

Mio. - Rec.

Mio.

Mio. - Plio.

Mio. - Rec.

* (Mio.)

Plio. - Rec.

*

$\because$

$*$

Plio. - Rec. 
Suchium (Protorotella) maxima SHUTo

Crepidula sp.

Xenophora (Tugurium) dunkeri MARTIN

Polinices (Glossaulax) reiniana (DUNKER) subsp.

Tectonatica janthostomoides KURODA and HABE

Eudolium oyamai SHUTO

Cassidaria n. sp.

Murex (Murex) cfr. verbecki MARTIN

M. (M.) cfr. djarianensis MARTIN

Columbarium pagodum (LESSON)

Nassaria magnifica (LISCHKE) n. subsp.

Granulifusus koyuanus SHUTO

Ancilla (Turrancilla) otukai SHUTo

Cancellaria (Merica) cfr. pristina Yokoyama

Fulgoraria (Psephaea) koshibensis OTUKA

F. (P.) sp.

Ancistrosyrinx osawanoensis TsUDA

Spirotropis subdeclivis (YoKoYAMA) n. subsp.

Surculites siogamensis NOMURA

Borsonia sp.

Asprella (Endemoconus) toyamaensis (TSUDA)

Ringicula (Ringiculina) doliaris (GouLD)
* (Mio.)

Plio.

Plio. - Pleist.

Mio. - Rec.

*

$\%$

Plio.

Plio.

Mio. - Rec.

$*$

*

$*$

Mio. - Plio.

Plio.

Mio.

*

Mio.

Mio.

Mio. - Rec.

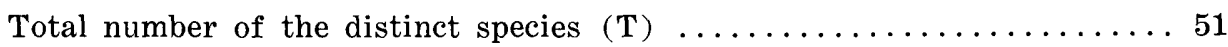

Number of the inidentified species $(\mathrm{Sp}) \ldots \ldots \ldots \ldots \ldots \ldots \ldots \ldots \ldots$

Number of the species known only from the Miyazaki group (Msp) . . 11

Number of the Oligocene to Miocene element .............. 2

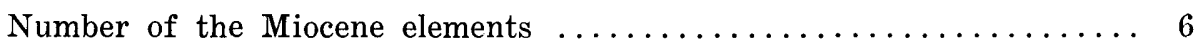

Number of the Miocene to Pliocene elements $\ldots \ldots \ldots \ldots \ldots \ldots \ldots 4$

Number of the Miocene to Recent elements $\ldots \ldots \ldots \ldots \ldots \ldots \ldots \ldots 11$

Number of the Pliocene elements ..................... 7

Number of the Pliocene to Pleistocene element $\ldots \ldots \ldots \ldots \ldots \ldots \ldots 1$

Number of the Pliocene to Recent elements $\ldots \ldots \ldots \ldots \ldots \ldots \ldots . \ldots 4$

Number of the species known only from Recent $\ldots \ldots \ldots \ldots \ldots \ldots, 1$

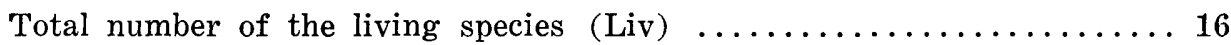

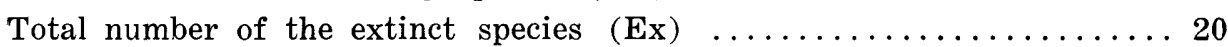

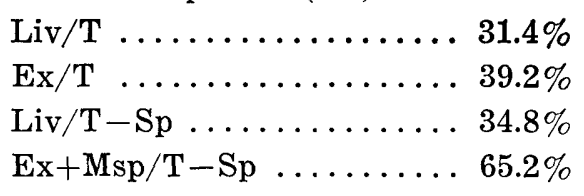

(3) The second fossil horizon (Yamaji horizon)

Ennucula cfr. akitana OTUKA

Acila (Acila) submirabilis MAKIYAMA

Saccella confusa kongiensis OTUKA
Mio.

Mio. - (Rec.)

Mio. 
Yoldia (Tepidoleda) naganumana (OTUKA) n. subsp. Acar plicata (DILlwYN)

Glycymeris rotunda (DUNKER)

Limopsis obliqua A. ADAMS

Pectunculina oblonga (A. ADAMs)

Pteria sp.

Chlamys sp.

Amussiopecten iitomiensis (OTUKA)

Lima (Acesta) goliath (SowERBY)

Crassatellites (Crassatellites) tsumaensis SHUTO

C. (Crassatina) oblongatus uchidanus (YoKoYAMA)

Scambra (Eucrassatella) sp.

Venericardia (Megacardita) megacostata SHUTo

Lucinoma annulata (REEVE)

L. sp.

Joannisiella cumingii (HANLEY)

Clinocardium subdecussatum SHUTO

Vepricardium kyushuense SHUTo

Nemocardium (Keenaea) samarangae (MAKIYAMA)

Callista (Callista) chinensis (HoLTEN)

C. (C.) roscida (Gould)

Venus (Ventricoloidea) foveolata miyazakiensis (SHUTo)

Dosinia (Phacosoma) nomurai OTUKA

D. sp.

Placamen tiara (DILlwYN)

Paphia (Paphia) exilis takaokaensis SHUTo

P. (P.) grata tsumaensis SHUTo

$P$. (P.) hirabayashii koyuensis ShUto

Solecurtes dunkeri KURODA

Merisca serricostata (TOKUNAGA)

Fabulina nitidula (DUNKER)

Cadella semitorta (SowERBY)

Solen sp.

Periploma pulchellum Hatai and Nisiyama

Myadora proxima (SMITH)

Cuspidaria (Cardiomya) n. sp.

Lischkeia alwinae (LISCHKE)

Calliostoma (Otukaia) kounjianum (YokoYaMA)

Suchium (Suchium) koyuense SHUTо

S. (S.) n. sp.

S. (Protorotella) hyugaensis SHUTo

Polinices (Glossaulax) reiniana (DUNKER) subsp.

Mammila simiae (Deshayes)

Uberella? sp.
* (Plio. - Pleist.)

Plio. - Rec.

Plio. - Rec.

Mio. - Rec.

Plio. - Rec.

Mio.

Mio. - Rec.

*

Plio.

*

Mio. - Rec.

Mio. - Rec.

*

$*$

Mio. - Rec.

Mio. - Rec.

Plio. - Rec.

*

Mio.

Plio. - Rec.

Mio. - Low. Plio.

*

* (Mio.)

Rec.

Plio. - Pleist.

Rec.

Rec.

Mio.

Rec.

*

Plio. - Rec.

Low. Plio.

*

*

* (Mio.)

Plio. - Pleist.

Rec. 
Tonna costata DESHAYES

Eudolium oyamai SHUTo

Ficus sp.

Cassidaria n. sp.

Cymatium cfr. tenuiliratus (LISCHKE)

Typhis japonicus A. ADAMS

Siphonalia tonohamaensis totomiensis MAKIYAMA

Nassaria magnifica LiSCHKE n. subsp.

Granulifusus koyuanus SHUTo

Nassarius (Hinia) caelatus (A. ADAMs) n. subsp.

Oliva (Oliva) mustellina LAMARCK

O. (O.) ispidula LINNÉ

Olivella (Olivella) consobrina LISCHKE

O. (O.) fulgurata (ADAMS and REEVE)

Ancilla (Baryspira) albocallosa okawai (YoKoYaMa)

Flugoraria (Fulgoraria) daviesi FultoN

F. (Psephaea) sp.

Uromitra nakamurai ugariensis MAKIYAMA

Ptychosyrinx n. sp.

Gemmula n. sp.

Bathytoma n. sp.

Turricula (Surcula) n. sp.

Spirotropis subdeclivis (YoкоYама) n. subsp.

Clavatula n. sp.

Psudoinquisitor pseudoprincipalis (YoKоYaMa) n. sp.

$P$. n. sp.

Thatcheria gradata (YoKoYAMA)

Myurella naumanni YoKoYAMA

Retusa sp.
Plio.

感

$\%$

Plio. - Rec.

Plio. - Rec.

Low. Plio.

$\%$

$*$

$\%$

Mio. - Rec.

Plio. - Rec.

Mio. - Rec.

Mio. - Rec.

Low. Plio.

Rec.

Low. Plio.

*

$*$

$*$

*

* (Plio.)

*

$\ddot{*}$

*

ㄸ.

Plio. - Rec.

Total number of the distinct species $(\mathrm{T}) \ldots \ldots \ldots \ldots \ldots \ldots \ldots \ldots \ldots$

Number of the inidentified species $(\mathrm{Sp}) \ldots \ldots \ldots \ldots \ldots \ldots \ldots \ldots \ldots \ldots \ldots$

Number of the species known only from the Miyazaki group (Msp) . . 26

Number of the Miocene elements $\ldots \ldots \ldots \ldots \ldots \ldots \ldots \ldots \ldots \ldots \ldots$

Number of the Miocene to Pliocene elements ................ 1

Number of the Miocene to Recent elements $\ldots \ldots \ldots \ldots \ldots \ldots \ldots \ldots 10$

Number of the Pliocene elements ..................... 6

Number of the Pliocene to Pleistocene elements .............. 2

Number of the Pliocene to Recent elements ................. 10

Number of the species known from only Recent $\ldots \ldots \ldots \ldots \ldots \ldots \ldots 6$

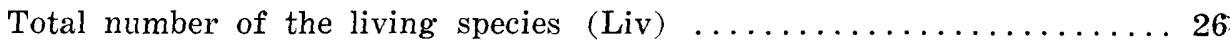

Total number of the extinct species $($ Ex $) \ldots \ldots \ldots \ldots \ldots \ldots \ldots \ldots \ldots \ldots$

$\operatorname{Liv} / \mathrm{T} \ldots \ldots \ldots \ldots \ldots \ldots . \ldots .2 \%$

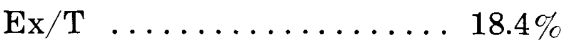

Liv $/ \mathrm{T}-\mathrm{Sp} \ldots \ldots \ldots \ldots \ldots 39.4 \%$ 


$$
\mathrm{Ex}+\mathrm{Msp} / \mathrm{T}-\mathrm{Sp} \ldots \ldots \ldots 60.6 \%
$$

2nd hor.

\begin{tabular}{|c|c|c|c|c|c|c|c|}
\hline \multirow[t]{7}{*}{ U. Oligo. } & L. Mio. & M. Mio. & U. Mio. & Plio. & Pleist. & Rec. & \\
\hline & & & & & & & 5 \\
\hline & & & & & & & 10 \\
\hline & & & & & & & 6 \\
\hline & & & & & & & 2 \\
\hline & & & & & & & 6 \\
\hline & & 16 & 16 & 20 & 2? & 26 & 40 \\
\hline
\end{tabular}

3rd hor.

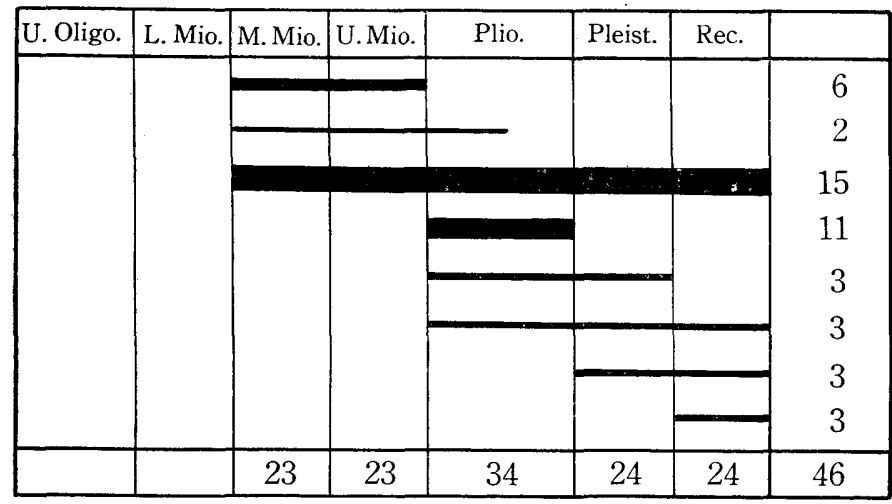

Text-fig. 10. Diagrammatic presentation of the composition of the molluscan assemblages of the second and the third horizons with regard to the known ranges of the species.

(4) The third fossil horizon (Tonogôri horizon)

Acila (Acila) submirabilis MAKIYAMA

Barbatia (Barbatirus) cometa (REEVE)

Pseudogrammatodon sp.

Glycymeris rotunda (DUNKER)

G. totomiensis MAKIYAMA

Limopsis obliqua A. ADAMS

Mimachlamys miniacea (LAMARCK)

Amusium n. sp.

Ostrea (Pycnodonta) musashiana (YoKoYAMA)

Crassatellites (Crassatellites) tosanus NomuRA

Venericardina (Megacardita) oyamai SHUTo

Thyasira tokunagai KURODA and HABE

T. sp.
Mio. - (Rec.)

Pleist. - Rec.

Plio. - Rec.

Low. Plio.

Mio. - Rec.

Rec.

*

Plio. - Rec.

Low. Plio.

$*$

Pleist. - Rec. 
Lucinoma annulata (REEVE)

Pillucina (Pillucina) pisidium (DUNKER)

$P$. (Sydlorina) cfr. yokoyamai OTUKA

Joannisiella cumingii (HANLEY)

Felaniella usta (GouLd)

Vepricardium kyushuense SHUTO

Nemocardium (Keenaea) samarangae (MAKIYAMA)

Callista (Callista) chinensis (HoLTEN)

Dosinia (Phacosoma) nomurai OTUKA

Paphia (Paphia) grata tsumaensis ShUto

Merisca cfr. serricostata (ToKUNAGA)

Macoma (Pseudometis) praerupta (SALISBURY)

Angulus sp.

Periploma besshoensis (YoKoYaMa) n. subsp.

Dentalium hexagonum GouLd

Siphonodentalium (Pulserum) ozawai (YoKoYaMA)

Calliostoma (Otukaia) kounjianum (YoKoYaMA)

Ethalia (Ethaliella) ? sp.

Calyptraea sp.

Crepidula cfr. isimotoi OTUKA

Turritella (Turritella) perterebra YoKoYama

Xenophora (Tugurium) dunkeri (MARTIN)

Polinices (Glossaulax) reiniana (DUNKER) subsp.

Uberella yokoyamai KURODA and HABE

Tectonatica janthostomoides KURODA and HABE

Tonna deshayesii REEVE

Eudolium oyamai SHUTo

E. sp. a

Apollon sp.

Murex (Murex) cfr. verbecki MARTIN

Columbarium pagodum (LESSON)

Babylonia areolata (LINK)

Siphonalia declivis tosaensis MAKIYAMA

S. dainitiensis MAKIYAMA

S. cfr. ikebei TsudA

Nassaria magnifica LISCHKE n. susbp.

Kelletia sp.

Cantharus wangwanensis NomurA

Buccinum sp.

Granulifusus koyuanus SHUTO

Nassarius sp.

Oliva (Oliva) mustellina LAMARCK

Olivella (Olivella) fulgurata (ADAMs and REEve)

Ancilla (Baryspira) albocallosa okawai (Yokoyama)
Mio. - Rec.

Mio. - Rec.

Mio.

Mio. - Rec.

Mio. - Rec.

*

Mio. - Rec.

Mio. - Rec.

Mio.

*

Plio. - Pleist.

Pleist. - Rec.

* (Mio.)

Mio. - Rec.

Plio.

Low. Plio.

Mio.

Upp. Mio. - Low. Plio.

Plio.

Plio. - Pleist.

Plio. - Pleist.

Mio. - Rec.

Rec.

*

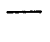

Plio.

Mio. - Rec.

Rec.

Low. Plio.

Low. Plio.

Mio.

*

Plio.

*

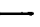

Mio. - Rec.

Mio. - Rec.

Low. Plio. 
Sydaphera spengleriana (DESHAYES)

Cancellaria (Merica) pristina (Yokoyama)

Turris cfr. kurodai MAKIYAMA

Bathytoma sp.

Turricula (Surcula) sobrina (YokoYama)

Ancistrosyrinx osawanoensis TSUDA

Cymatosyrinx sp.

Inquisitor (Inquisitor) jeffreysii (SMITH)

Borsonia sp.

Retusa sp.
Mio. - Rec.

Mio. - Plio.

Low. Plio.

Low. Plio.

Mio.

Plio. - Rec.

Total number of the distinct species $(\mathrm{T}) \ldots \ldots \ldots \ldots \ldots \ldots \ldots \ldots \ldots \ldots$

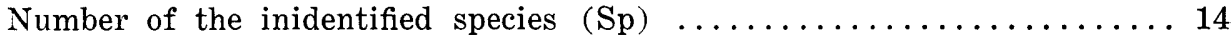

Number of the species known only from the Miyazaki group (Msp) $\ldots . \quad 7$

Number of the Miocene elements .....................6 6

Number of the Miocene to Pliocene elements ............... 2

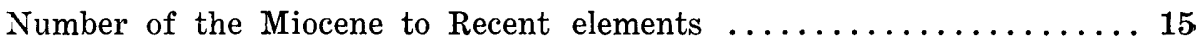

Number of the Pliocene elements ..................... 11

Number of the Pliocene to Pleistocene elements ............... 3

Number of the Pliocene to Recent elements ................ 3

Number of the Pleistocene to Recent elements .............. 3

Number of the species known only from Recent $\ldots \ldots \ldots \ldots \ldots \ldots . . . .3$

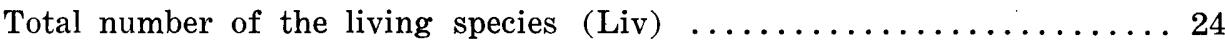

Total number of the extinct species $(\mathbf{E x}) \ldots \ldots \ldots \ldots \ldots \ldots \ldots \ldots \ldots 22$

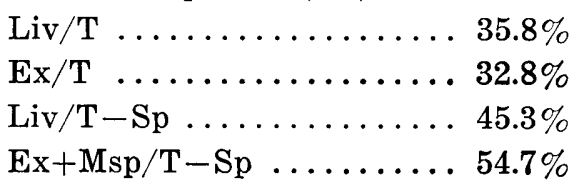

(5) The fourth fossil horizon (Hagenoshita horizon)

Acila (Acila) divaricata (HINDS)

Mio. - Rec.

Yoldia (Tepidoleda) naganumana (ОTUKA) n. subsp.

* (Plio. - Pleist.)

Anadara (Diluvarca) tricenicosta (NYST)

Plio. - Rec.

Hawaiarca uwaensis (YoKoYAMA)

Plio. - Rec.

Glycymeris rotunda (DUNKER)

Plio. - Rec.

$G$. totomiensis MAKIYAMA

Low. Plio.

Cucullaea granulosa JoNES

Mio. - Rec.

Limopsis obliqua A. ADAMS

Pectunculina oblonga (A. ADAMS)

Mio. - Rec.

Oblimopa forskalii (A. ADAMS)

Plio. - Rec.

Cryptopecten vesiculosus (DUNKER)

Plio. - Rec.

Aussiopecten praesignis (YoKOYAMA)

Mio. - Rec.

Anomia lischkei DeUtzenberg and Fischer

Low. Plio.

Crassatellites (Crassatellites) tosanus Nomura

Mio. - Rec.

Venericardia (Megacardita) panda (YoKоYAMA)

Low. Plio.

Low. Plio. 
V. (M.) granulicostata NomURA

Low. Plio.

Nemocardium (Keenaea) samarangae (MAKIYAMA)

Mio. - Rec.

Veremolpa scabra (HANLEY)

Myadora proxima (SмIтн)

Plio. - Rec.

Erodona cfr. amurensis (SCHRENCK)

Rec.

Dentalium hexagonum GoULD

Rec.

Mio. - Rec.

D. (Antalis) totomiensis MAKIYAMA

Low. Plio.

Siphonodentalium (Pulserum) ozawai (YoKoYAMA)

Plio. - Rec.

Calliostoma (Otukaia) kounjianum (YoKoYAMA)

Low. Plio.

Suchium (Suchium) obsoletum arenarium MAKIYAMA

Low. Plio.

Teinostoma pusillum (C. B. ADAMs)

Pleist. - Rec.

Starkeyna sobrina (A. ADAMS)

Bittium binodulosum YoKoYAMA

Pleist. - Rec.

Turritella (Haustator), n. sp.

Orectospira excelsa (YoKoYAMA)

Calyptraea yokoyamai KURoDA

Crepidula cfr. isimotoi OTUKA

Plio.

*

Plio. - Rec.

Pleist. - Rec.

Mio.

Melanella (Melanella) sp.

M. (Balcis) musta (Yoкоуама)

Pyramidella (Tibera) 2 spp.

Turbonilla (Tropaeas) sp.

T. (Turbonilla) 2 spp.

Odostomia (Odostomia) hilgendorfi (LESSON)

O. (O.) $2 \mathrm{spp}$.

Syrnola sp.

Kleinella (Leucotina) sp.

Menestho (Oscilla) sp.

Philippia cingulum (KIENER)

Polinices (Glossaulax) reiniana (DUNKER) subsp.

Plio. - Rec.

P. (G.) didyma (RödING)

P. (G.) didyma bicolor (BoRN)

Mammila simiae (DESHAYES)

Uberella yokoyamai KURODA and HABE

U. rufa (BoRN)

Tectonatica janthostomoides KURODA and HABE

$T$. andoi NOMURA

Volva sp.

Gyrineum bitubercularis (LAMARCK)

Plio. - Pleist.

Cymatium cfr. exaratus (REEVE)

Poirieria azami KURODA

Plio. - Pleist.

Mio. - Rec.

Pleist. - Rec.

Plio. - Rec.

Plio. - Pleist.

Plio. - Rec.

Mio. - Rec.

Plio.

Chicoreus pliciferoides KURODA

Murex (Murex) cfr. djarianensis MARTIN

Plio. - Rec.

Plio. - Rec.

Rec.

Plio. - Rec.

Plio.

Ceratoderma adunka (SoWERBY)

Pyrene bicinctella (YокоYAMA)

Plio. - Rec.

Plio. 
P. lischkei (SмITH)

Babylonia elata (YoKoyama)

Siphonalia declivis declivis YOKOYAMA

S. declivis tosaensis MAKIYAMA

S. declivis YokoYama n. subsp. a

S. declivis Yoкочама n. subsp. b

S. spadicea fuscolineata (PEAsE)

Nassaria magnifica LischKE

N. magnifica LisCHKE n. subsp.

N. lischkei MaKIYAMA

Granulifusus dualis (YoKoYAMA)

G. matsumotoi SHUTo

Hemifusus ternatanus (GMELIN)

Nassarius (Hinia) festivus (Powy) n. subsp.

Oliva (Oliva) mustellina LAMARCK

Olivella (Olivella) spretoides YoKOYAMA

O. (O.) baetica CARPENTER

Ancilla (Baryspira) albocallosa okawai (YokoYAMA)

A. (B.) oyamai SHuTo

Fulgoraria (Fulgoraria) cancellata KURODA and HABE

F. (Psephaea) kamakuraensis OTUKA

Lyria mizuhonica koyuana (YokoYAMA)

Mitra (Nebularia) hanleyana (DUNKER)

Trigonostoma kurodai MAKIYAMA

Sydaphera spengleriana (DESHAYES) n. subsp.

Cancellaria (Merica) pristina (YoKoYAMA)

C. (Cancellaria) reevei laticostata (LöBBECKE)

Marginella novemprovincialis (YOKOYAMA)

M. flaccida (YoKoYAMA)

Turris kurodai MAKIYAMA

Ptychosyrinx n. sp.

Gemmula granosa (HELBRING) n. subsp.

Bathytoma striatotuberculata (YoKoYAMA)

Micantapex n. sp.

Turricula (Orthosurcula) pervirgo (YoKoYAMA)

T. (Surcula) sobrina (YokоYAмA)

T. (S.) n. sp.

Ancistrosyrinx osawanoensis TSUDA

Cosmasyrinx n. sp.

Spirotropis subdeclivis (YOKOYAMA)

Leucosyrinx coreanica (ADAMS and REEVE)

Clavatula (Paradrillia) djocdjocartae serrana FISCHER

C. (P.) astuta (YOKOYAMA)

C. (P.) dainichiensis (YOKOYAMA)
Plio. - Rec.

Low. Plio.

Low. Plio.

Low. Plio.

*

*

Plio. - Rec.

Plio. - Rec.

*

Plio. - Rec.

Low. Plio.

*

Plio. - Rec.

*

Mio. - Rec.

Plio. - Pleist.

Plio. - Rec.

Low. Plio.

*

Rec.

Plio.

* (Plio.)

Plio. - Rec.

Low. Plio.

$\%$

Mio. - Plio.

Plio. - Rec.

$\%$

*

Low. Plio.

*

$*$

Plio.

*

Low. Plio.

Low. Plio.

\%

Mio.

*

Plio.

Plio. - Rec.

Plio.

Low. Plio.

Low. Plio. 
Cymatosyrinx rinsuikawaensis NoMURA

C. osawanoensis TSUDA

Inquisitor (Inquisitor) jeffreysii (SMITH)

Pseudoinquisitor totomiensis ugariensis MAKIYAMA

Tomopleura subdifficilis MAKIYAMA
Low. Plio.

Mio.

Plio. - Rec.

Low. Plio.

Low. Plio.

4th hor.

\begin{tabular}{|c|c|c|c|c|c|c|c|}
\hline \multirow[t]{7}{*}{ U.Oligo. } & L. Mio. & M. Mio. & U. Mio. & Plio. & Pleist. & Rec. & \\
\hline & & & & & & & 4 \\
\hline & & & & & & & $\begin{array}{r}1 \\
11\end{array}$ \\
\hline & & & & & & & 35 \\
\hline & & & & & & & 25 \\
\hline & & & & & & & 7 \\
\hline & & 16 & 16 & 76 & 44 & 47 & 91 \\
\hline
\end{tabular}

5th hor.

\begin{tabular}{|c|c|c|c|c|c|c|c|}
\hline U. Oligo. & L. Mio. & M.Mio. & U.Mio. & Plio. & Pleist. & Rec. & . \\
\hline & & & & & & & 7 \\
\hline & & & & & & & 17 \\
\hline & & & & & & & 7 \\
\hline & & & & & & & 5 \\
\hline & & & & & & & 2 \\
\hline & & 7 & 7 & 34 & 22 & 21 & 41 \\
\hline
\end{tabular}

6th hor.

\begin{tabular}{|c|c|c|c|c|c|c|c|}
\hline \multirow[t]{6}{*}{ U. Oligo. } & L. Mio & M. Mio & U. Mio & Plio. & Pleist. & Rec. & \\
\hline & & & & & & & 1 \\
\hline & & & & & & & 15 \\
\hline & & & & & & & 26 \\
\hline & & & & & & & 2 \\
\hline & & & & & & & 10 \\
\hline & & 16 & 16 & 63 & 36 & 44 & 73 \\
\hline
\end{tabular}

Text-fig. 11. Diagrammatic presentation of the composition of the molluscan assemblages of the fourth, fifth, and sixth horizons with regard to the known ranges of the species. 
T. yokoyamai (MAKIYAMA)

Borsonia n. sp.

$B$. sp.

Etrema hayasakai NomURA

Lienardia (L.) n. sp.

Antiguraleus sp.

Asprella (Asprella) comatosa (PILSBRY)

Myurella (Pervicacia) recticostata (YoKoYama)

Strioterebrum (Punctoterebra) makiyamae TSUDA

S. (P.) ozawai (YoKоYAMA)

"Terebra" $2 \mathrm{spp}$.

Ringicula (Ringiculina) doliaris (GoULD)

R. (R.) teramachii HABE

Retusa sp.

Acteocina (Decolifer) oyamai HABE
Low. Plio.

$*$

Low. Plio.

*

Rec.

Plio.

Mio.

Plio.

Mio. - Rec.

Rec.

Rec.

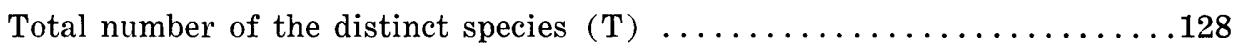

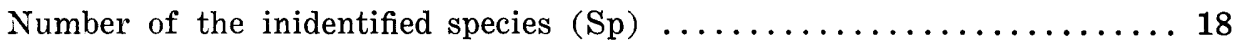

Number of the species known only from the Miyazaki group (Msp) . .. 18

Number of the Miocene elements $\ldots \ldots \ldots \ldots \ldots \ldots \ldots \ldots \ldots \ldots \ldots$

Number of the Miocene to Pliocene elements $\ldots \ldots \ldots \ldots \ldots \ldots \ldots 1$

Number of the Miocene to Recent elements $\ldots \ldots \ldots \ldots \ldots \ldots \ldots 12$

Number of the Pliocene elements ..................... 35

Number of the Pliocene to Pleistocene elements .............. 4

Number of the Pliocene to Recent elements $\ldots \ldots \ldots \ldots \ldots \ldots \ldots 25$

Number of the Pleistocene to Recent elements .............. 4

Number of the species known only from Recent $\ldots \ldots \ldots \ldots \ldots \ldots . \ldots 7$

Total number of the living species (Liv) $\ldots \ldots \ldots \ldots \ldots \ldots \ldots \ldots 4$

Total number of the extinct species $(\mathrm{Ex}) \ldots \ldots \ldots \ldots \ldots \ldots \ldots \ldots$

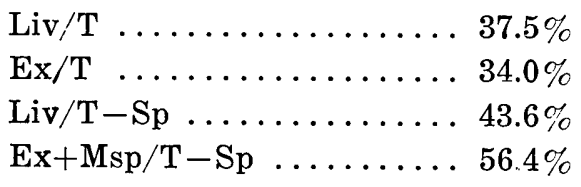

(6) The fifth fossil horizon (Nihonmatsu horizon)

Nucula (Nucula) paulula (A. ADAMs)

Cucullaea granulosa (JoNAS)

Pleist. - Rec.

Glycymeris rotunda (DUNKER)

Mio. - Rec.

Limopsis obliqua A. ADAMS

Plio. - Rec.

Pectunculina oblonga (A. ADAMS)

Mio. - Rec.

Amussiopecten praesignis (YoKoYAMA)

Plio. - Rec.

Anomia sp.

Joannisiella lunaris (YoKoYAMA)

Low. Plio.

Bellucina civica (YoKOYAMA)

Pleist. - Rec.

Myadora proxima (SмITH)

Mio. - Rec.

Rec. 
Siphonodentalium nipponicum MAKIYAMA

Dentalium (Antalis) totomiensis MAKIYAMA

Pseudoliotia micans (A. ADAMS)

Turritella (Turritella) perterebra YoKOYAMA

Orectospira excelsa YoKoYAMA

Bittium binodulosum YoKoYAMA

Turbonilla sp.

Melanella (Balcis) musta (YoKoYAMA)

Polinices (Mammilaria) sagamiensis (PILSBRY)

P. (Glossaulax) didyma bicolor (PHILIPPI)

Uberella yokoyamai KURODA and HABE

$U$. n. sp.

Tectonatica janthostomoides KURODA and HABE

Cymatium cfr. tenuiliratus (LISCHKE)

Chicoreus pliciferoides KURODA

Siphonalia declivis declivis YoKoYama

S. declivis tosaensis MAKIYAMA

Nassaria lischkei KuRODA

Rabyloniae elata (YoKoYAMA)

Granulifusus dualis (YoKоYAMA)

G. matsumotoi SHUTO

Nassarius (Hinia) caelatus dainitiensis MAKIYAMA

$N$. (H.) caelatus (A. ADAMS) n. subsp.

Ancilla (Baryspira) albocallosa okawai (Yokoyama)

Olivella (Olivella) spretoides YoKoYama

Marginella novemprovincialis (YoKoYAMA)

M. flaccida (YOKOYAMA)

Gemmula granosa (HeLBRING) n. subsp.

Bathytoma striatotuberculata (YOKOYAMA)

Spirotropis subdeclivis (YoKOYAMA)

Leucosyrinx coreanica (A. ADAMS and REEVE)

Clavatula (Paradrillia) dainichiensis (YoKoYAMA)

Tomopleura subdifficilis MAKIYAMA

Cymatosyrinx rinsuikawaensis NoMURA

Bellaspira (Lyromangilia) semicarinata (PILSBRY)

Acteon kirai $\mathrm{HABE}$

Pupa strigosa (GoULD)

Ringicula (Ringiculina) doliaris (Gould)
Low. Plio.

Low. Plio.

Plio. - Rec.

Upp. Mio. - Rec.

Plio. - Rec.

Plio.

Plio. - Pleist.

Mio. - Rec.

Pleist. - Rec.

Plio. - Pleist.

$*$

Mio. - Rec.

Plio. - Rec.

Plio. - Rec.

Low. Plio.

Low. Plio.

Plio. - Rec.

Low. Plio.

Low. Plio.

*

Low. Plio.

*

Low. Plio.

Plio. - Pleist.

*

$*$

*

Plio.

Plio.

Plio. - Rec.

Low. Plio.

Low. Plio.

Low. Plio.

Plio. - Rec.

Rec.

Pleist. - Rec.

Mio. - Rec.

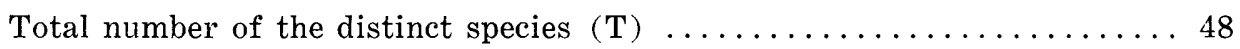

Number of the inidentified species $(\mathrm{Sp}) \ldots \ldots \ldots \ldots \ldots \ldots \ldots \ldots \ldots 2$

Number of the species known only from the Miyazaki group (Msp) . .. 4

Number of the Miocene to Recent elements ............. 7

Number of the Pliocene elements $\ldots \ldots \ldots \ldots \ldots \ldots \ldots \ldots \ldots \ldots \ldots$

Number of the Pliocene to Pleistocene elements $\ldots \ldots \ldots \ldots \ldots \ldots . \ldots$ 
Number of the Pliocene to Recent elements $\ldots \ldots \ldots \ldots \ldots \ldots \ldots \ldots$

Number of the Pleistocene to Recent elements $\ldots \ldots \ldots \ldots \ldots \ldots \ldots \ldots \ldots$

Number of the species known only from Recent $\ldots \ldots \ldots \ldots \ldots \ldots \ldots \ldots$

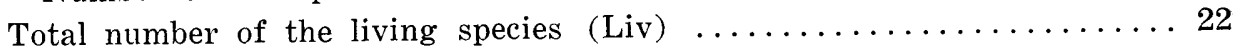

Total number of the extinct species $(\mathrm{Ex}) \ldots \ldots \ldots \ldots \ldots \ldots \ldots$

\begin{tabular}{|c|c|}
\hline $\mathrm{Liv} / \mathrm{T} \ldots$ & $45.8 \%$ \\
\hline $\mathrm{Ex} / \mathrm{T} \ldots$ & $39.5 \%$ \\
\hline $\mathrm{Liv} / \mathrm{T}-\mathrm{Sp} \ldots$ & $45.7 \%$ \\
\hline $\mathrm{Ex}+\mathrm{Msp} / \mathrm{T}-\mathrm{Sp}$ & $54.3 \%$ \\
\hline
\end{tabular}

(7) The sixth fossil horizon (Tôriyama horizon)

Acila (Acila) divaricata (HINDS)

A. (Truncacila) minuta (MAKIYAMA)

Saccella confusa (HANLEY)

Anadara (Scapharca) castellata (YoKoyAMA)

A. sp.

Glycymeris rotunda (DUNKER)

G. totomiensis MAKIYAMA

Veletuceta nakamurai (MAKIYAMA)

Limopsis obliqua A. ADAMS

Pectunculina oblonga (A. ADAMs)

Atrina (Atrina) penna (REEVE)

Volsella nitida (REEVE)

Gloripallium satowi (YoKoYAMA)

G. aurantiacus (SOWERBY)

Mimachlamys cfr. miniacea (LAMARCK)

Cryptopecten vesiculosus (DUNKER)

Patinopecten taiwanus NomURA

Amussiopecten praesignis (YokoYAMA)

Palliolum (Delectopecten) macrocheiricola $\mathrm{HABE}$

Ostrea (Pycnodonta) musashiana (YoKoYAMA)

O. (Crassostreo) gigas (THUMBERG)

Crassatellites (Crassatellites) takanabensis SHUTO

Cardita sp.

Venericardia (Megacardita) panda (YoKoYAMA)

V. (M.) ferruginosa (A. ADAMS and REEvE)

V. (M.) granulicostata NomURA

Thyasira (Conchocele) nipponica YABE and NoMURA

Lucinoma annulata (REEVE)

Dinocardium braunsi (ToKUNAGA)

Fulvia mutica (REEVE)

Nemocardium (Keenaea) samarangae (MAKIYAMA)

Callista (Callista) chinensis (HoLTEN)

Dosinia (Phacosoma) troscheli (LISCHKE)
Mio. - Rec.

Plio.

Mio. - Rec.

Plio.

Plio. - Rec.

Low. Plio.

Low. Plio.

Mio. - Rec.

Plio. - Rec.

Plio. - Rec.

Rec.

Low. Plio.

Plio. - Rec.

Rec.

Mio. - Rec.

Low. Plio.

Low. Plio.

Rec.

Plio. - Rec.

Mio. - Rec.

*

Low. Plio.

Mio. - Rec.

Low. Plio.

Mio. - Plio.

Mio. - Rec.

Plio. - Pleist.

Mio. - Rec.

Mio. - Rec.

Mio. - Rec.

Plio. - Rec. 
D. (Dosinorbis) bilunulata (GRAY)

Clementia (Clementia) papyracea GRAY

Placamen tiara (DILwYN)

Mercenaria stimpsoni (GouLD)

Veremolpa mindanensis (SMITH)

Paphia (Paphia) takanabensis SHUTo

Coelomactra antiquata (SPENGLER)

Solecurtes dunkeri KURODA

Mysella n. sp.

Cuspidaria (Cuspidaria) hindsiana (A. ADAMs)

Dentalium (Paradentalium) octangulatum (DoNOVAN)

D. (Antalis) totomiensis MAKIYAMA

Siphonodentalium (Siphonodentalium) nipponicum MAKIYAMA

S. (Pulserum) ozawai (YoKоYAMA)

Trochus sp.

Lischkeia n. sp.

Turcicula argenteonitens convexiuscula (YoKоYAMA)

Turcica sp.

Calliostoma (Tristichotrochus) shinagawaense cipangoanum (YoKoYAMA)

Suchium (Suchium) suchiense subsuchiense MAKIYAMA

Bittium sp.

Xenophora (Tugurium) dunkeri MARTIN

Polinices (Mammilaria) sagamiensis (PILSBRY)

P. (Glossaulax) reiniana (DUNKER) subsp.

Tonna canaliculata LINNÉ

Rapana aff. rapiformis (BORN)

Murex (Murex) djarianensis MARTIN

Cerastoderma adunka (SOWERBY)

Pyrene bicinctella (YoKoYAMA)

Babylonia elata (YoKoYAMA)

Plicifusus sp.

Nassaria magnifica LISCHKE

Siphonalia mikado Melville

Granulifusus dualis (YoKoYAMA)

G. matsumotoi SHUTо

Fusinus simplex SMITH

$F$. perplexus A. ADAMS

Hemifusus sp.

Oliva (Oliva) mustellina LAMARCK

Ancilla (Baryspira) albocallosa okawai (YoKoyama)

Fulgoraria (Fulgoraria) cancellata KURODA and HABE

Lyria mizuhonica mizuhonica MAKIYAMA
Plio. - Rec.

Mio. - Rec.

Plio. - Rec.

Plio. - Rec.

Rec.

*

Rec.

Rec.

*

Rec.

Mio. - Rec.

Low. Plio.

Low. Plio.

Plio. - Rec.

*

Plio.

Plio.

Plio.

Plio.

Mio. - Rec.

Plio. - Pleist.

Rec.

Rec.

Plio.

Plio. - Rec.

Plio.

Low. Plio.

Plio. - Rec.

Plio. - Rec.

Low. Plio.

*

Plio. - Rec.

Plio. - Rec.

Mio. - Rec.

Low. Plio.

Rec.

Low. Plio. 
Turris kurodai MaKIYAMA

Gemmula granosa (HELBRING)

Bathytoma striatotuberculata (YokoYama)

B. luedorf (LISCHKE)

Turricula (Orthosurcula) australis (RoIssY)

T. (Surcula) sobrina (YOKOYAMA)

Leucosyrinx coreanica (ADAMS and REEVE)

Spirotropis subdeclivis (YoKoYAMA)

Terebra insulindae FISCHER

Philine scalpta (A. AdAMs)
Low. Plio.

Mio. - Rec.

Plio.

Plio. - Rec.

Rec.

Low. Plio.

Plio. - Rec.

Plio.

Plio.

Rec.

Total number of the distinct species $(\mathrm{T}) \ldots \ldots \ldots \ldots \ldots \ldots \ldots \ldots \ldots$

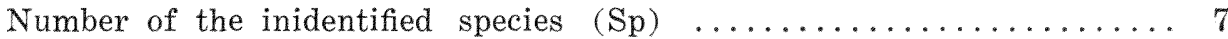

Number of the species known only from the Miyazaki group (Msp) .... 4

Number of the Miocene to Pliocene elements ................ 1

Number of the Miocene to Recent elements .................. 15

Number of the Pliocene elements ...................... 26

Number of the Pliocene to Pleistocene elements ............... 2

Number of the Pliocene to Recent elements ................. 19

Number of the species known only from Recent ............... 10

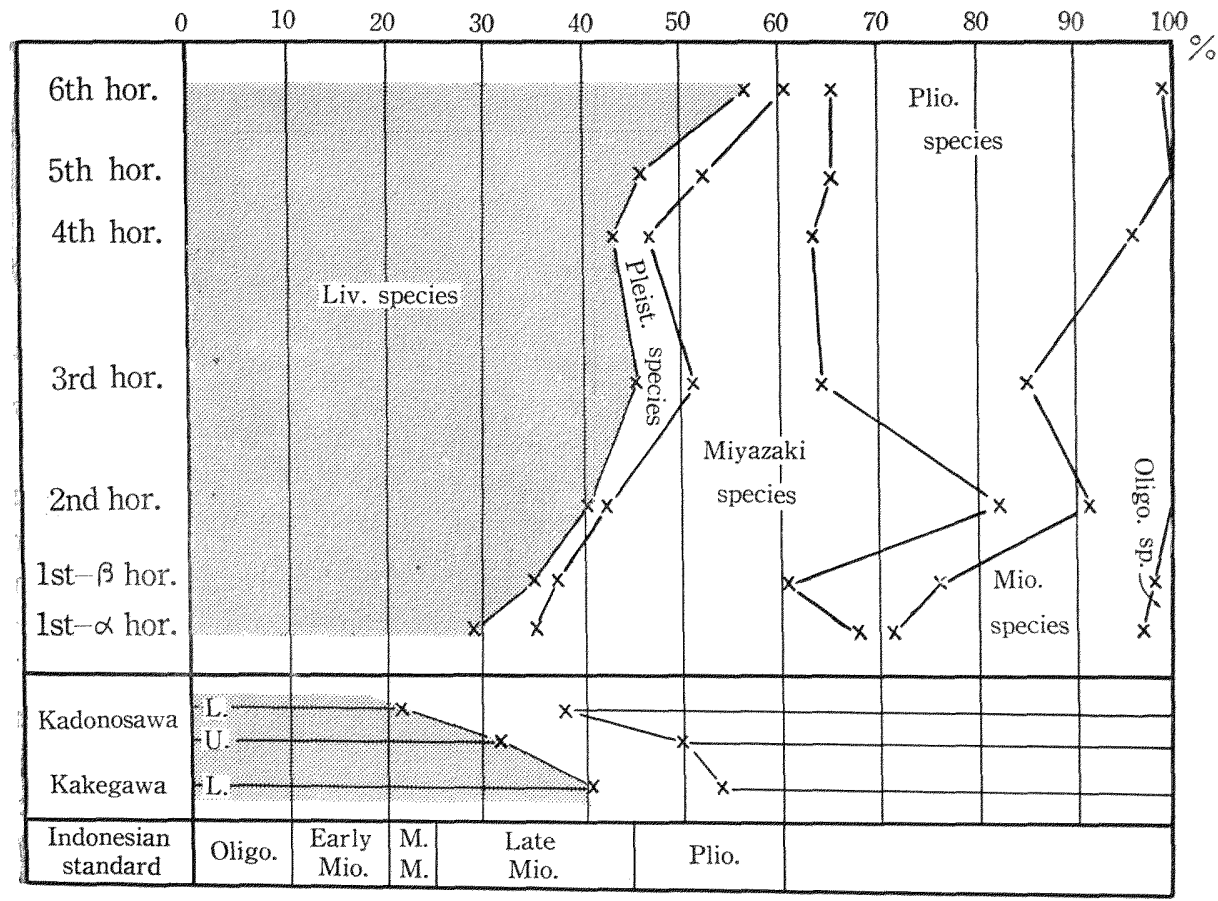

Text-fig. 12. Percentage analysis of the molluscan assemblages of the Miyazaki group.

Total number of the species (100\%) is divided into living, Pleistocene, Oliocene, Miocene, and Oligocene elements and those only known from the Miyazaki group. 
Total number of the living species (Liv) $\ldots \ldots \ldots \ldots \ldots \ldots \ldots \ldots 44$

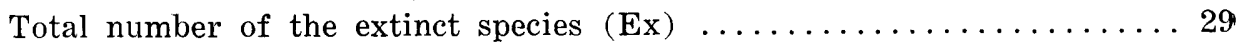

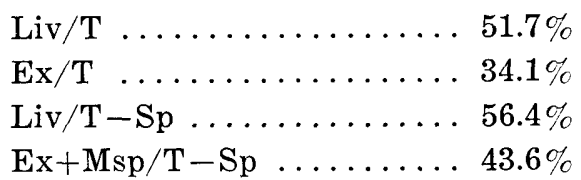

(8) Dating by the percentage method

K. MARTIN (1913, 161-173), WANNER and HAHN (1935, 222-273), and Oostingh $(1938,17-33)$ revised the Lyellian method so as to make it serviceable for the dating of the tropic faunas. According to them, the percentages of the number of the living species of the tropic Neogene faunas are 40 to 55 percent for Pliocene, 30 percent for Late Miocene, and 15 to 20 percent for Early Miocene. These Indonesian criteria may be applicable to the rough estimation of the geologic age of the Miyazaki group, because the fossil assemblages of that group, as already stated, are tropic to subtropic.

Percentages of the number of the living species of respective fossil horizons of the Miyazaki group are 28 percent for the first- $a, 35$ percent for the first- $\beta, 39$ percent for the second, about 45 percent for the third, fourth, and fifth, and 56 percent for the sixth horizon. Accordingly the Miyazaki group is correlated to the early Late Miocene to Pliocene of Indonesia by the percentage method. The detailed correlation is given in the following section.

II-Biological sequence and the zonation in the Miyazaki group

(1) Faunal succession and the geologic range of some important species in the Miyazaki group

It is generally accepted in Japan that the "middle Miocene" transgression, one of the greatest transgressions in the geological history of Japan, was taken its place towards "late Miocene" age by the gradual regression, which resulted not only the limitation of the sea but also the deepening of it. The "Pliocene" transgression followed this regression of short duration. Reflecting this history of transgression and regression the marine faunal succession in this time span was illustrated by the tropical shallow water fauna, the cold and deep water fauna, and the "Pliocene" fauna. The last one was differentiated into the cold shallow water fauna of Japan sea province and the subtropic shallow water one of the region surrounding southwest Japan.

In the Miyazaki group the faunal change from the first- $\alpha$ to the sixth horizon is not abrupt, but rather gradual. The forerunner of many "Pliocene" elements. appeared at the lower horizons and a remarkable number of the "Miocene" elements left their survivors and off-shoots at the higher horizons. This is also indicated by the examination of the exotic elements, the percentage of which falls down gradually from 10 percent (first-a) to 2 percent (third) and then rises to 6 percent (fourth). Thereafter it again gradually changes as indicated in the text-fig. 13. This indicates that the third horizon represents some transi- 
Table 2. Geological range of the important molluscan species of the Miyazaki group and its biostratigraphic zonation.

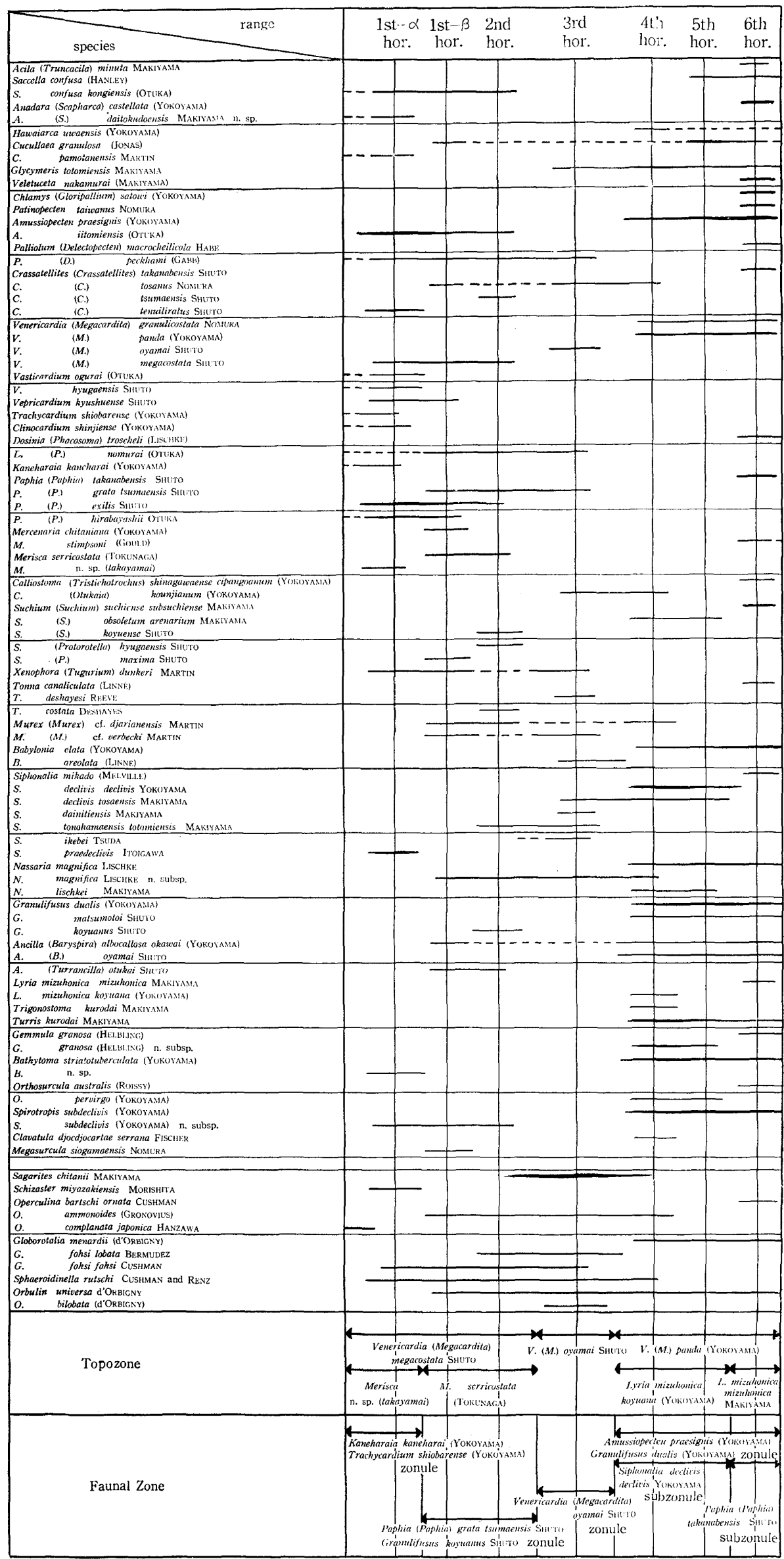


tion phase, although the change is very gradual and no evidence suggests the falling of the water temperature at this transition stage as is verified at the central and north Japan.

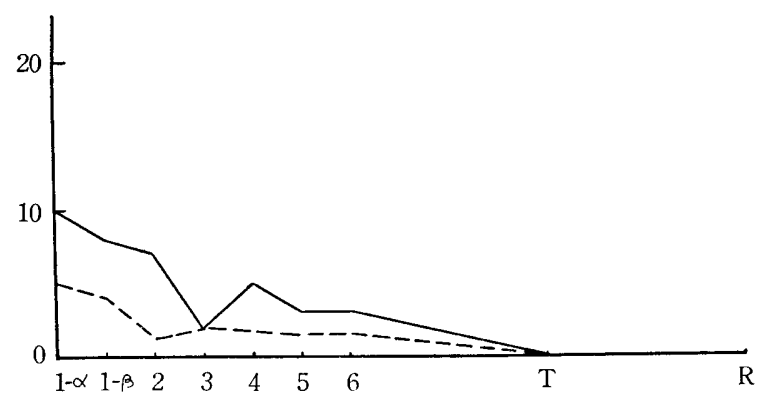

Text-fig. 13. Change in the percent of the exotic elements in the late Caenozoic marine molluscan faunas of the Miyazaki district. broken line....extinct element; solid line....exotic element. $1-\alpha, 1-\beta, 2,3,4,5,6 \ldots$ respective horizons of the Miyazaki group; T...Tôriyama formation (Mid. Pleisto.); R....recent.

The noticeable change in the composition of the faunas in the successional stages is the decrease of the cardiids and the increase of the buccinids, pectinids, and other indigenous elements. It is very interesting to clarify the relation between this tendency of the faunal succession and the geological range of the individual elements. As already noted, the fossil localities of the Miyazaki group are not scattered evenly in the strata, but rather concentrated in several limited parts. Consequently even the most prolific species occurs at intervals in the vertical sections and the geologic range of each species can not be traced continucusly in the strict sense of the words. The following table is a somewhat generalized range chart of some important species (table 2).

(2) Zonules and Topozones in the Miyazaki group

The examination of the geologic range of each species from the Miyazaki group shows that (a) some species are very long ranging and consequently not available for zoning, that (b) very short-lived species occur rather abundantly characterizing a certain horizon, and that (c) the greater parts of the species range in the moderate duration such as from first- $a$ to second, first- $\beta$ to fourth, fourth to six, etc. According to the frequency of the examples, they may be divided into three groups, (a) group limiting to the first- $\alpha$ horizon, (b) group ranging from the first- $\beta$ to second, and (c) group ranging from the fourth to sixth horizon. (c) group, in turn, is subdivided into $\left(c_{1}\right)$ ranging from the fourth to the fifth horizon and $\left(c_{2}\right)$ limiting to the sixth horizon. Many species of the (b) and (c) group range up and down to the third horizon. In other words the third horizon represents the transitional stage of the biological sequence with regards to both faunal succession and the geologic range of the individual elements. On the basis of the above mentioned facts the following zonules are distinguished. 
Kanaharaia kaneharai-Trachycardium shiobarense zonule .......... (a) Paphia grata tsumaensis-Granulifusus koyuanus zonule ............ (b) Amussiopecten praesignis-Granulifusus dualis zonule ............(c)

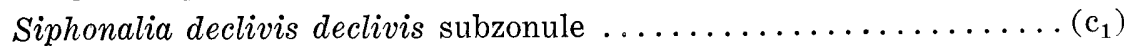

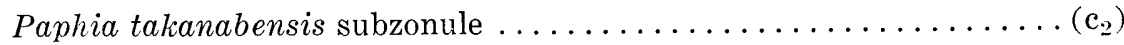
Megacardita oyamai zonule ........Transitional zonule between (b) and (c)

Amussiopecten praesignis-Granulifusus dualis zonule is characterized by the assemblage of Amussiopecten praesignis (YokoYAMA), Granulifusus dualis (YoKOYAMA), Venericardia (Megacardita) panda (YoKoYAMA), Babylonia elata (YoKoYAMa), Pyrene bicinctella (YoKoYAma), Bathytoma striatotuberculata (Yokoyama), Placamen tiara (DIllwyn), Spirotropis subdeclivis (YoKoyama), and Leucosyrinx coreanica (ADAMS and REEVE). Paphia takanabensis subzonule is characterized by Paphia (Paphia) talkanabensis SHuTo, Crassatellites takanabensis Shuto, Acila minuta MakiYama, Anadara castellata (Yokoyama), Veletuceta nakamurai (MAKIYAMA), Gloripallium satowi (YoKoYAMA), G. aurantiacus (Sowerby), Patinopecten taiwanus Nomura, Calliostoma (Tristichotrochus) shinagawaense cipangoanum (YoKoYAMA), Orthosurcula australis (RoISSY), Siphonalia mikado MELVILL, and Bathytoma luehdorfi (LISCHKE) and represents the hemera of Amussiopecten praesignis, Gloripallium satowi, and Anadara castellata. Siphonalia declivis declivis subzonule is characterized by Siphonalia declivis declivis YoKoYAMA, Orectospira excelsa (YoKoYAMA), Trigonostoma kurodai MAKIYAMA, Marginella novemprovincialis (YoKoYAMA), $M$. flaccida (Yokoуama), Orthosurcula pervirgo (Yokoyama), Clavatula (Paradrillia) dainichiensis (YoKoYAMA), Cymatosyrinx rinsuikawaensis NomuRA, Tomopleura suzdifficilis MAKIYAMA, Etrema hayasakai Nomura etc. Megacardita oyamai zonule is featured by the overlapping of younger and older elements and by the diagnostic elements such as Venericardia (Megacardita) oyamai SHuTo, Periploma besshoensis (YoKоYамA) n. subsp. etc.

Paphia grata tsumaensis-Granulifusus koyuanus zonule is characterized by Crassatellites (Crassatellites) tsumaensis Sнuto, Paphia (Paphia) grata tsumaensis SHUTo, Granulifusus koyuanus SHUTo, Merisca serricostata (ToKUNAGA), Thatcheria gradata (YokoYAMA), Ancilla (Turrancilla) otukai SHuTo, Lischkeia alwinae (LISCHKE), Galeodea n. sp. etc. Kaneharaia kaneharai-Trachycardium shiobarense zonule is characterized by the so-called Miocene elements such as Anadara daitokudoensis MAKIYAMA, A. valentula (YokoYAmA), Cucullaea pamotanensis MARTIN, Crassatellites tenuiliratus SHUTo, Vasticardium ogurai (OTUKA), Trachycardium shiobarense (YoKoYAMA), Clinocardium shinjiense (YoKOYAMA), Bonartemis suketoensis (OTUKA), Kaneharaia kaneharai (YoKoYAMA), Siphonalia praedeclivis ITOIGA, Surculites yokoyamai OTUKA.

Phacosoma nomurai OTUKA, Saccella confusa kongiensis (OTUKA), Amussiopecten iitomiensis (OTUKA), and Megacardita megacostata SHUTo range through the last mentioned two zonules altogether.

This tendency of periodicity in the biostratigraphic change is not only exemplified by the sequence of the faunal assemblages, but also illustrated by the 
lineal evolution of particular organic groups. For example the lineage from Amussiopecten iitomiensis (OTUKA) (first-a to second) to A. praesignis (YokoYAMA) (fourth to sixth), Venericardia (Megacardita) megacostata SHuTo (first-a to second) to $V$. (M.) panda (Yokoyama) (fourth to sixth) via $V$. (M.) oyamai Shuto (third), Suchium (Suchium) koyuense Shuto and S. (Protorotella) (first- $\beta$ to second) to $S$. (S.) suchiense (Yokoyama) and obsoletum MakiYama (fourth to sixth), Granulifusus koyuanus SHUTo (first- $\beta$ to third) to G. dualis (Yokoyama) and matsumotoi SHuTo (fourth to sixth), Spirotropis subdeclivis n. subsp. (first-a to second) to S. subdeclivis subdeclivis (YokoYama) (fourth to sixth), Bathytoma n. sp. (first-a to second) to B. striatotuberculata (YokoYAMA) (fourth to sixth), and Lyria mizuhonica koyuana YokoYAMA (fourth) to L. mizuhonica mizuhonica MaKIYAMA (sixth). These are represented biochronologically by three successive topozones (Moore, 1957), i.e. Venericardia (Megacardita) panda (YokoyAma), $V$. (M.) oyamai Shuto, and $V$. (M.) megacostata SHUTo topozone in descending order, of which panda topozone and megacostata topozone are subdivided respectively into Lyria mizuhonica mizuhonica MAKIYAMA and L. mizuhonica koyuana YoKoYama topozone and Merisca serricostata (ToKUNAGA) and $M$. n. sp. (takayamai) topozone.

This surprising concordance between the faunal and lineal successions suggests that the lineal evolution and the faunal change of the mollusks were controled primarily by the same factors.

As indicated in table 2 the zonules based on the higher foraminifers and planktonic foraminifers also rather harmonize with the preceding conclusion. That is to say, Operculina complanata japonica HANZAWA, O. ammonoides (GRONOVIUS), and $O$. bartschi ornata CUsHMAN have respective range of the first- $a$, first- $\beta$ to third, and sixth horizon, which respectively correspond to Kaneharaia kaneharai-Trachycardium shiobarense zonule, Megacardita oyamai zonule + Paphia tsumaensis - Granulifusus koyuanus zonule, and Paphia takanabensis zonule. While the planktonic foraminiferal zonules, Globorotalia menardii zonule and $G$. fohsi zonule respectively, correspond to Amussiopecten praesignis-Granulifusus dualis zonule and to Megacardita oyamai zonule to Paphia grata tsumaensisGranulifusus koyuanus zonule.

These lead the conclusion that the biostratigraphically significant boundary of the elementary order lies just below the fourth horizon, and the subordinately important boundaries lie between the first- $\alpha$ and the first- $\beta$ horizon, between the second and the third, and between the fifth and the sixth. These boundaries may be useful for the intraprovincial correlation.

\section{III-Correlation}

(1) - Comparison with the Kakegawa fauna

The standard of the Pliocene bio-stratigraphy of our country were established on the Kakegawa group in Shizuoka Prefecture by MakiYama (1940, 641-649), who proposed the stages Dainitian and Ketienzian. Prior to this proposal the palaeontological studies of the mollusks of the Kakegawa group had been carried 
out by Yokoyama (1926, 313-364) and MAKiYAMA (1927, 1-147) independently. Since that time several contributions have been added to the biostratigraphy and molluscan palaeontology of the Kakegawa group. The majority of them, however, did not deal with the general problems of the faunas but with the special subject or the description of part of the fauna, without giving the fundamental alteration to MAKIYAMA's scheme. Hereupon I adopted MAKIYAMA's result for the basis of the comparison of the molluscan faunas of the Kakegawa group with those of the Miyazaki group.

MAKIYAMA described 181 species from the lower part of the Kakegawa group, concluding that the age of this fauna (Dainitian fauna) corresponds to the European Plaisancian or Indian Pontian. The molluscan assemblages obtained from the Dainichi (Dainiti) and Tennô sand member respectively contain the living species by 41.6 percent and 52.7 percent of the total number of the species. The former corresponds to the figures of the second, third, fourth, and fifth horizon of the Miyazaki group and the latter to the figure of the sixth horizon.

The comparison of the elements of the assemblages reveals that the Dainichi assemblage closely resembles those of the above mentioned horizons with numerous species in common as indicated in the following.

5 species in common with the second horizon $(5 / 66=7.6 \%)$

10 species in common with the third horizon $(10 / 53=18.8 \%)$

22 species in common with the fourth horizon $(22 / 109=20.2 \%)$

13 species in common with the fifth horizon $(13 / 46=28.2 \%)$

19 species in common with the sixth horizon $(19 / 78=24.4 \%)$

The Tennô assemblage also resembles those of the upper horizons of the Miyazaki group with the large percentages of the common species.

10 species in common with the third horizon $(10 / 53=18.8 \%)$

23 species in common with the fourth horizon $(23 / 109=21.1 \%)$

10 species in common with the fifth horizon $(10 / 46=21.9 \%)$

34 species in common with the sixth horizon $(34 / 78=43.6 \%)$

Comparison of these figures leads to the conclusion that the Tennô sand member of the Kakegawa group is correlated to the first horizon of the Miyazaki group with least doubt. The Dainichi assemblage, on one hand, resembles those of the third to sixth horizon in the percentage of the living element and also in the number of the common species. However the assemblage of the third horizon contains many "Miocene" elements, which are entirely absent in those of the higher horizon of the Miyazaki group and in the Dainichi assemblage. Among the assemblages of the upper three horizons of the Miyazaki group the Dainichi assemblage shows the closest affinity to that of the fifth horizon. On the other hand the assemblage of the fifth horizon does not differ essentially from that of the fourth horizon, although the former contains the younger elements as Nucula paulula (A. AdAMs), Joannisiella lunaris (YokoYama), Pseudoliotia micans (A. AdAMS), Acteon kirai HABE, and Pupa strigosa (Gould). Accordingly I am inclined to correlate the Dainichi member to the fourth to the fifth horizon of the Miyazaki group. This agrees with the result based on the ranges of the 
important index species.

Following is the list of the species which are common between the lower Kakegawa and the Miyazaki groups.

Acila (Truncacila) minuta (MAKiYAMA), Acila (Acila) divaricata (Hinds), Limopsis obliqua A. AdAMs, Anadara (Scapharca) castellata (YoKoYAMA), Glycymeris rotunda DUNKER, G. totomiensis MAKIYAMA, Veletuceta nakamurai (MAKIYAMA), Atrina pectinata (Linné), Ostrea (Pycnodonta) musashiana (YoKoYAMa), Amussiopecten praesignis (Yokoyama), Anomia lischkei DAUTzenBERG and Fischer, Crassatellites (Crassatina) oblongatus uchidanus (YokoYAMA), Venericardia (Megacardita) panda (YoKoyama), Fulvia mutica (ReEve), Nemocardium (Keenaea) samarangae (MAKIYAMA), Dosinia (Phacosoma) troscheli (LISCHKe), D. (Dosinorbis) bilunulata (GRAY), Mercenaria chitaniana (YoKoyAma), Placamen tiara (Dillwyn), Callista (Callista) chinensis (Holten), Solecurtes divaricatus (LISCHKE), Panope japonica REEVE, Dentalium totomiensis MAKIYAMA, Siphonodentalium nipponicum MAKIYAMA, Lischkeia alwinae (LISCHKE), Suchium (Suchium) suchiense subsuchiense MAKIYAMA, S. (S.) obsoletum arenarium MaKIYAMa, Turritella perterebra YokoYama, Tectonatica janthostomoides Kuroda and Habe, Polinices (Mammilaria) sagamiensis Pilsbry, Murex (Murex) cfr. djarianensis MARTIN, Babylonia elata (Yokoyama), Siphonalia dainitiensis Makiyama, S. declivis declivis Yokoyama, S. mikado Melvill, Nassaria magnifica LischKe, $N$. lischkei MakiYama, Nassarius (Hinia) caelatus dainitiensis MAKIYAMA, Lyria mizuhonica MAKIYAMA, Uromitra nakamurai ugariensis MAKIYAMA, Oliva (Oliva) mustellina LAMARCK, Olivella (Olivella) spretoides YoKoYAMA, O. (O.) fulgurata (AdAMS and REEve) O. (O.) consobrina (LisChKe), Ancilla (Baryspira) albocallosa okawai (YoKoyama), Sydaphera spengleriana (Deshayes), C. (Merica) pristina (YoKoyama), Trigonostoma kurodai MAKIYAMA, Turris kurodai MakiYAMA, Gemmula granosa (Helbling), Tomopleura yokoyamai MakiYama, T. subdifficilis MakiYama, Bathytoma luedorfi (Lischke), Spirotropis subdeclivis (YoKoYama), Turricula (Surcula) sobrina (YoкоYама), Inquisitor jeffresysii (SMITH), I. totomiensis ugariensis MAKIYAMA, Clavatula dainichiensis YокоYама, Ringicula doliaris (GOULD).

Among the lower Kakegawa species of YokoYAMA 37 species are in common with the mass assemblage of the upper three horizons (4th, 5th, 6th) of the Miyazaki group, and these species are mostly included in the foregoing list except for Philippia cingulata (KIENER), Cymatium tenuiliratus (LISCHKE), Mercenaria stimpsoni (Gould), and Pectunculina oblonga (A. ADAMs).

(2) --Comparison with the Tônohama fauna in Shikoku

The Tônohama group, distributed along the southeastern coast of Kôchi City in Shikoku, was palaeontologically studied by YokoYAMA (1926, 365-368, 1929, 9-17), Makiyama (1927, 21-22), Nomura (1937, 67-90), Katto, NaKamura, and Takayanagi (1953, 1-15), and Katto and OzaKi (1955, 1-7). According to Katto the Tônohama group consists of three formations, the Anai, Nahari, and Nobori in descending order. Each of the formations is about $100 \mathrm{~m}$. in thickness, and 
the marine Anai formation is underlain conformably by the non-marine Nahari, which is in turn underlain unconformably by the marine Nobori formation. All the fossil localities described by Yokoyama, MakiYama, and Nomura belong to the Anai formation.

The molluscan assemblage of the Anai formation as a whole closely resembles those of the Amussiopecten praesignis-Granulifusus dualis zonule of the Miyazaki group. The comparison of NomurA's faunal list of the Anai formation, which is the most detailed among the available reports, with those of the fossil horizons of the Miyazaki group indicates the very high percentage of the species in common. The number of the species in common are

4 species for the first- $a$ horizon $(4 / 60=6.6 \%)$

10 species for the first- $\beta$ horizon $(10 / 46=21.7 \%)$

10 species for the second horizon $(10 / 66=15.1 \%)$

15 species for the third horizon $(15 / 53=28.3 \%)$

32 species for the fourth horizon $(32 / 109=29.3 \%)$

17 species for the fifth horizon $(17 / 46=36.9 \%)$

29 species for the sixth horizon $(29 / 78=37.2 \%)$

According to the same author the Anai assemblage consists of 214 species, of which 158 ( 74 percent) are living. The geological ranges of the elements of the assemblage are as following: 2 species for Oligocene to Recent; 4 species for Miocene to Pliocene; 33 species for Miocene to Recent; 31 species for Pliocene only; 82 species for Pliocene to Recent; 4 species for Pleistocene only; 2 species for Pleistocene to Recent; 20 species only for Recent; 17 new species.

The molluscan assemblage of the Nobori formation consists of 39 species, of which 16 species (41 percent) are living. It also contains the typical Miocene indices such as Nuculana yokoyamai KuRoda, Periploma pulchellum HataI and NisiYama, Palliolum (Delectopecten) peckhami (GABB), and Sagarites, resembling the assemblages of the middle horizons of the Miyazaki group. The number of the species common between the Miyazaki group and th Nobori formation are the following:

2 species for the first- $a$ horizon $(2 / 60=3.3 \%)$

7 species for the first- $\beta$ horizon $(7 / 46=15.2 \%)$

9 species for the second horizon $(9 / 66=13.6 \%)$

9 species for the third horizon $(9 / 53=16.9 \%)$

9 species for the fourth horizon $(9 / 109=8.2 \%)$

4 species for the fifth horizon $(4 / 46=8.7 \%)$

7 species for the sixth horizon $(7 / 78=8.9 \%)$

On the grounds of the percentage of the living species, the number of the species in common, the number of the index species, and the stratigraphic relation, I am inclined to correlate the Anai formation to the fifth and the sixth horizons. and the Nobori formation to the second and the third horizons of the Miyazaki group.

(3) Comparison with the Shimajiri fauna in Okinawa 
The mollusks of the Shimajiri group in Okinawa Island were described by NomURA and JiNBo (1935, 226-266) who correlated the group to the Tennô sand member of the Kakegawa group on the foundation of the faunal affinity with 14 species in common. The Shimajiri assemblage consists of 83 living species (78 percent), 11 extinct ones (10 percent), 12 new species, and 8 unidentified ones. The species in common with the Miyazaki group are Anadara (Diluvarca) tricenicosta (NYST), Cucullaea granulosa (JoNAS), Amussiopecten praesignis (YoKoYAMA), Chlamys (Gloripallium) satowi (YoKoYAMA), Joannisiella cumingii (HANley), Callista chinensis (Holten), Myadora proxima Smith, Dentalium hexagonum Gould, Siphonodentalium nipponicum MakiYama, Pseudoliotia micans (A. ADAMS), Polinices (Glossaulax) didyma (RoEDING), Inquisitor jeffreysii (SMITH), and Pupa strigosa (Gould).

The number of the species in common with the respective horizons of the Miyazaki group are as follows:

2 species in common with the third horizon $(2 / 53=3.8 \%)$

5 species in common with the fourth horizon $(5 / 109=4.6 \%)$

7 species in common with the fifth horizon $(7 / 46=15.2 \%)$

4 species in common with the sixth horizon $(4 / 78=5.1 \%)$

In spite of the high percentage of the living species, the Shimajiri assemblage shows the closest appearance not to the uppermost horizon of the Miyazaki group, but to the fifth one. However, since the Shimajiri group and the sixth horizon of the Miyazaki group represent altogether the hemera of such important index species as Gloripallium satowi and Amussiopecten praesignis with abundant occurrence, the two units are necessarily correlated.

(4) - Comparison with the Neogene mollusks of the Kwanto district surroundingTokyo

The Neogene strata of the Kwanto district with extensive distribution have repeatedly and minutely investigated by many authors. Bôsô peninsula, southeastern part of the district, is one of the standard area for the Pliocene and Pleistocene stratigraphy of Japan. Unfortunately the Pliocene and Miocene strata of the area bear only a few molluscan fossils.

According to OTUKA $(1947,94)$ the Amatsu formation yields the small assemblage of the mollusks, which consists of 7 species including 3 living ones (43 percent). The species in common with the Miyazaki group are Amussiopecten praesignis (YoKoYAMA), Ostrea (Pycnodonta) musashiana (YoKoYAMA), Conchocele nipponica YABE and NomURA, Palliolum (Delectopecten) peckhami (GABB), and Gloripallium miurensis (YoKoYAMA). These are of course far from the concrete base for the reliable correlation. Considering, however, the said geological range of these significant species, it is better to conclude that the Amatsu formation is correlated with third to fourth horizon of the Miyazaki group.

From the overing Kiyosumi subgroup 17 species of the mollusks were reported (OTUKA, 1949, 295-309). This assemblage consists of the deep water forms and includes 8 living species (47 percent). Only two species of the Kiyosumi assembl- 
age are in common with that of the Miyazaki group. They are Fulgoraria (Psephaea) kosibensis OTUKA and Limopsis obliqua A. ADAMs, both of which occurred from the first- $\beta$ horizon of the Miyazaki group. This fact may not have any chronologic significance but suggests the resemblance in the ecological condition of the two units, and accordingly exact correlation cannot be carried out from the available molluscan material.

From the Ofuna formation in Miura peninsula, OTUKA $(1949,310)$ obtained 31 molluscan species, of which the following 8 species are in common with the Niiyazaki assemblage. They are Acila (Acila) divaricata (HINDs), Ostrea gigas Thumberg, Lucinoma annulata (REeve), Fulvia mutica (REeve), Tectonatica jansostomoides KURODA and HABE, Sydaphera spengleriana (DESHAYES), Clavatula (Paradrillia) dainichiensis (YoKоYAMA), and Inquisitor jeffreysii (SмrтH). Two and four species of the eight are included respectively in the assemblage of the fifth and the sixth horizons. Five species including such Dainichi elements as Sydaphera spengleriana and Clavatula (Paradrillia) dainichiensis occur in the fourth horizon.

The molluscan assemblage of the overlying Koshiba formation consists of 95 species and contains only 19 species in common with the Miyazaki group. The important common elements between both units are Yoldia (Tepidoleda) naganumana OTUKA, Anadara (Scapharca) castellata (YokoYama), Ostrea (Pycnodonta) musashiana (YoкоYама), Gloripallium miurensis (YoKoYAMA), Lima (Acesta) goliath (SMITH), Conchocele nipponica YABE and Nomura, Keenaea samarangae (MAKIYAMA), Bittium binodulosum YokoYAma, and Inquisitor jeffreysii (SMITH).

The common 19 species between two stratigraphic units contain not only any apparent Miocene element, but also few of the typical Dainichi elements, and the greater part of them occurs in the sixth horizon of the Miyazaki group. Furthermore the percentages of the living species in the of una and the Koshiba formation are about 60 according to the revised data of Ogose $(1959,96)$. That is to say the ôfuna and the Koshiba faunas rather resemble the faunule of the Paphia takanabensis subzonule, but the former two contain much less Dainichi elements and much more living species than the latter. Consequently the former may be somewhat younger than the latter, the top of the Miyazaki group.

The Nakatsu group, developed along the middle course of the Sagami river in Kanagawa Prefecture, yields a large molluscan assemblage. According to SUZUKI (1932, 49-70, 97-132) the assemblage obtained from the lower part of the group consists of 109 species, which contain 66 living elements ( 60.5 percent). 35 species of this assemblage are in common with those of the Miyazaki group including the following important species: Yoldia (Tepidoleda) naganumana Otuka, Portlandia (Portlandella) japonica (AdAMs and ReEve), Glycymeris totomiensis (MAKIYAмa), Veletuceta nakamurai (MAKIYAMA), Gloripallium miurensis (YokoYama), Venericardia (Megacardita) panda (Yokoyama), Felaniella usta (GOULD), Joannisiella cumingii (HANLEY), Trachycardium shiobarense (YokoYama), Keenaea samarangae (MAKIYAMA), Mercenaria chitaniana (YoKo- 
YAMA), Suchium (Suchium) suchiense subsuchiense MAKIYAMA, S. (S.) obsoletum arenarium MAKIYAMA, Turritella perterebra YoкоYамA, Olivella (Olivella) spretoides YoKoYAma, Siphonalia declivis declivis YoKoyama, and Clavatula (Paradrillia) dainichiensis (YoKoYama).

The assemblage of the upper Nakatsu group consists of 60 species and contains 41 living form (68.3 percent). 17 species of the assemblage are in common with the Miyazaki group, including the important elements: Yoldia (Tepidoleda) naganumana OTUKA, Cryptopecten vesiculosus (DUNKER), Felaniella usta (GoULD), Conchocele nipponica YABE and Nomura, Keenaea samarangae (Makiyama), Panope japonica A. Adams, and Inquisitor jeffreysii (Smith).

The number of species of the lower and the upper Nakatsu group in common with the assemblages of the respective horizons of the Miyazaki group are tabulated below.

assemblage of the lower Nakatsu group

7 species in common with the first- $\alpha$ horizon $(7 / 60=11.6 \%)$

7 species in common with the first- $\beta$ horizon $(7 / 46=15.3 \%)$

9 species in common with the second horizon $(9 / 66=16.6 \%)$

10 species in common with the third horizon $(10 / 53=18.8 \%)$

13 species in common with the fourth horizon $(13 / 109=11.9 \%)$

8 species in common with the fifth horizon $(8 / 46=17.4 \%)$

17 species in common with the sixth horizon $(17 / 78=21.8 \%)$

assemblage of the upper Nakatsu group

8 species in common with the fourth horizon $(8 / 109=7.3 \%)$

2 species in common with the fifth horizon $(2 / 46=4.3 \%)$

8 species in common with the sixth horizon $(8 / 78=10.3 \%)$

Considering the percentage of the living species and the frequency of the "Pliocene elements", the lower Nakatsu group may be safely correlated to the Amussiopecten praesignis-Granulifusus dualis zonule of the Miyazaki group and the upper Nakatsu may be younger in age.

The Tertiary strata are extensively developed also in Jôban coal field, north Kwanto and divided into the following three groups: the Naigô [=Shiramizu], Yunagaya, and Taga in ascending order, of which the latter two groups have been considered to be Miocene in age. The subdivisions of the Miocene groups and their moliuscan elements in common with the assemblages of the Miyazaki group are listed below:

Taga group-Saccella confusa kongiensis (OTUKA), Periploma pulchellum Hatai and Nisiyama, Lucinoma acutilineata Conrad, Panope japonica A. ADAMS, Tectonatica janthostomoides KURODA and HABE.

Yunagaya group

Misawa formation-Turritella perterebra YoKоYAMA, Lucinoma acutilineata CONRAD

Honya formation-Conchocele nipponica YABE and NomURA, Lucinoma acutilineata Conrad, Periploma pulchellum Hatai and Nisiyama. 
Kamenowo and Mizunoya formation-Conchocele nipponica YABE and NoMURA, Lucinoma acutilineata CONRAD, Periploma besshoensis (YoKoYAMA).

Goyasu formation-none.

The Jôban Miocene mollusks, as a whole, represent rather the deep fauna comprising the species of lesser number, and consequently these figures do not result any reliable basis for the correlation of the stratigraphic units of this area to the Miyazaki group. However the second horizon of the latter may be approximately correlated to the Taga group, if the significance of the geologic ranges of such leading species as Saccella confusa kongiensis and Periploma pulchellum is evaluated critically.

(5) - Comparison with the Miocene faunas of northeast Japan

Ninoye district of Iwate Prefecture in northeast Honshû is one of the classical locality of the Miocene and Pliocene biostratigraphy in Japan. In this area the Yotsuyaku, Kadonosawa, and Suyenomatsuyama groups are developed in ascending order.

According to ОTUKA (1934, 566-638), the lower Kadonosawa fauna consists of 50 identified species, of which 11 (22 percent) are living and 36 (72 percent) are the Miocene elements. The upper Kadonosawa and the Suyenomatsuyama fauna consists of 22 and 15 species respectively. The former contains the living species by 32 percent and the Miocene elements by 50 percent. The common species between these faunas and those of the Miyazaki group are as follows:

Suyenomatsuyama fauna

3 species in common with the first- $a$ horizon

1 species in common with the sixth horizon

Venericardia (Megacardita) ferruginosa ADAMs and ReEve, Panope japonica A. ADAMs, and Epitonium (Boreoscala) nagamiensis OTUKA

upper Kadonosawa fauna

3 species in common with the first-a horizon

1 species in common with the first- $\beta$, the second, the third, and the fouorth horizon

Acila (Acila) submirabilis MakiYama, Ostrea gigas Thumberg,

Lucinoma acutilineata CoNRAD, and Panope japonica A. ADAMs.

lower Kadonosawa fauna

3 species in common with the first- $a$ horizon $(6.5 \%)$

4 species in common with the first- $\beta$ and second horizon ( 6.0 and $6.6 \%)$

2 species in common with the third and fourth horizon (1.8 and $3.8 \%$ )

Saccella confusa kongiensis (OTUKA), Ostrea gigas THUMBERG, Clinocardium shinjiense (Yokoyama), Callista chinensis Holten, Dosinia (Phacosoma) nomurai OTUKA, Polinices (Glossaulax) didyma (RoEDING), Olivella (Olivella) consobrina LISCHKE, Sydaphera spengleriana (DESHAYES), and Surculites (Megasurcula) yokoyamai OTUKA.

The number of the species in common between the two areas is, thus, surpri- 
singly small. This is of course natural in the case of the Suyenomatsuyama fauna, because it is featured by the cold water elements contrasting to the warm water faunas of the Miyazaki group. Although there are such important Miocene index species as Saccella confusa kongiensis, Clinocardium shinjiense, Dosinia (Phacosoma) nomurai, and Surculites (Megacurcula) yokoyamai in the 7 species common between the lower Kadonosawa group and the basal bed of the Miyazaki group, the former contains the Miocene elements by 72 percent, and the latter contains them by 29 percent (or 54 percent if the new species from this horizon are added as the Miocene elements). The figure, 54 percnt, is as large as the figure from the upper Kadonosawa fauna (50 percent). The meagerness of the species in common between the last mentioned two units may be the reflection of the separation of the palaeobiogeographical provinces about $1200 \mathrm{~km}$. apart from northeast to southwest each other. In conclusion I am inclined to correlate the lowest part of the Miyazaki group with the upper half of the Kadonosawa group.

The Miocene strata named the Natori group are distributed in the adjacent area of Sendai City, Miyagi Prefecture. This group is divided into four formations, the Takadate andesite, Moniwa, Hatadate, and Tsunaki formations in ascending order, and the latter three yield molluscan fossils. The Monwia, Hatadate, and Tsunaki assemblage consists respectively of 86 species including the living species by about 32 percent, 24 species with the living one by 33 percent, and 24 species with the living one by 29 percent. The number of the species of each fauna in common with the assemblages of the Miyazaki group are as follows:

Moniwa formation

$6,8,5,5,2,1$, and 3 species respectively in common with the first- $\alpha$, first- $\beta$, second, third, fourth, fifth, and sixth horizons. They are altogether 14 as listed below.

Acila (Acila) submirabilis MakiYama, Lima (Acesta) goliath (Sмrth), Ostrea gigas THUMBERG, Joannisiella cumingii (HANLEY), Felaniella usta (Gould), Lucinoma acutilineata CONRAD, Trachycardium shiobarense (YoKoYAMA), Callista chinensis (HoLten), Mercenaria chitaniana (YoKoYAMa), Dosinia (Phacosoma) nomurai OTUKA, Kaneharaia kaneharai (YoKoYAMA), Panope japonica A. AdAMs, Tectonatica janthostomoides KURODA and HABE, and Surculites (Megasurcula) siogamensis NoMURA.

Hatadate formation

$3,3,2,1$, and 1 species respectively in common with the first- $a$, first- $\beta$, second, third, and fourth horizon.

Lima (Acesta) goliath (SMITH), Lucinoma acutilineata CoNRAD, Clinocardium shinjiense (Yokoyama), Mercenaria chitaniana (Yokoyama), Panope japonica A. ADAMs, Tectonatica janthostomoides KURODA and HABE.

Tsunaki formation

2,1 , and 1 species in common with the first- $\alpha$, first- $\beta$, and second horizon. Lucinoma acutilineata CONRAD, Callista chinensis (HoLteN), Kaneharaia 


\section{kaneharai (YoKoYAMA).}

The examination of the molluscan assemblages of the Natori and the Miyazaki group suggests that the exact comparison can not be expected because of the paucity of the index species common to both stratigraphic units. The faunal discrepancy of this sort may due to the difference in the palaeo-biogeographical conditions of two groups as in the case of the preceding Kadonosawa group.

(6) - Comparison with the Miocene faunas of northwest Japan

The Neogene strata of the "geosynclinal province" along Japan Sea coast have been studied extensively by many geologists and palaeontologists, because the strata form the most important producing oil fields in this country. The molluscan fossils occur abundantly from the so-called Pliocene formation and rarely from the Miocene. The Pliocene mollusks, called Omma-Manganji fauna, consist purely of the cold water elements, and utterly differ from the warm water Kakegawa fauna, the latter of which includes same molluscan species as the upper three horizons of the Miyazaki group. The Miocene mollusks from the oil province show the close affinity to the Miocene faunas of northeast Honshû above mentioned, and accordingly the correlation of it with those of the Miyazaki group based on the molluscan materials alone can not be exactly done.

In the Izumo province, the western end of the oil Tertiary belt, the thick Neogene strata are extensively developed. In the standard section they are divided into eight formations, which are, according to TAI (1959, 265-395) the Hata, Kawai, Kuri, Omori, Kimachi, Fusina, Matsuye, and Tsunôzu formation in ascending order. The study on the molluscan palaeontology of this area was lead by M. Yokoyama (1923, 1-9), and thereafter several results have been offered. Among the eight formations the Fusina formation is richest in molluscan fossils in which 50 species containing 17 living species (34 percent) were distinguished by S. Nomura and K. HataI (1939, 1-9). The following 11 species are in common with the faunas of the Miyazaki group.

Acila (Acila) submirabilis MakiYama, Felaniella usta (Gould), Lucinoma acutilineata CONRAD, Keenaea samarangae (MAKIYAMA), Clinocardium shinjiense (Yokoyama), Trachycardium shiobarense (Yokoyama), Dosinia (Phacosoma) nomurai Otuka, Mercenaria chitaniana (Yokoyama), Panope japonica A. Adams, Polinices (Glossaulax) didyma (RoEdING), Fulgoraria sinziensis NomURA and HataI ( $=F$. daviesi Fulton).

9 of the above noted 11 species are known from the basal bed of the Miyazaki group. On the basis of the faunal affinity the Fusina formation is preliminarily correlated with the Paphia grata tsumaensis-Granulifusus koyuanus zonule to Kaneharaia kaneharai-Trachycardium shiobarense zonule of the Miyazaki group.

(7) - Comparison with the Neogene faunas of Formosa

In Formosa the Tertiary formations have been diligently studied from two standpoints, namely, from the tectonic interest and the oil prospecting. However the available palaeontological results are not enough for the general consideration except for the latest results on the foraminiferal faunas. Only a few formations 
have been extensively studied for molluscan palaecntology, one of which is the Byoritsu beds.

Both of the independent results of Yokoyama (1928, 1-112) and Nomura (1933, 1-106; 1936, 53-228) indicate that the Byoritsu beds can be correlated without any vital error with the Amussiopecten praesignis-Granulifusus dualis zonule, especially with the Paphia takanabensis subzonule of the Miyazaki group on the basis of the frequent occurrence of such common index species to both the beds as Gloripallium satowi (YoKoYama), Patinopecten taiwanus Nomura, Amussiopecten praesignis (YoKoYAMA), Venericardia (Megacardita) panda (YoKoyAMA), V. (M.) granulicostata NomurA, Tectonatica andoi Nomura, Olivella spretoides YokoYama, Turricula sobrina (YoKoYAMA), Cymatosyrinx rinsuikawaensis Nomura, and Etrema hayasakai Nomura.

The upper Arisan assemblage consists of 14 identified species, of which Venericardia (Megacardita) panda (YokoYAMA), Gloripallium satowi (YoKoYAMA), Amussiopecten praesignis (YoKoYAMA) and other two species are in common with the first horizon of the Miyazaki group. Both of the fifth and the fourth horizons yields three species in common with the upper Arisan. The Kaizan assemblage also consists of 14 identified species, of which 3 including "Amussiopecten praesignis" are in common with the fourth to sixth horizons of the Miyazaki group. Considering the geologic range of these index species, the so-called Upper Miocene beds in Formosa may be correlated with the fourth horizon of the Miyazaki group and represent the basal Pliocene. The other "Miocene" formations in Formosa have not been thoroughly studied for molluscan palaeontology and even a rough comparison with the Miyazaki group is difficult.

\section{(8)-Comparison with the Neogene faunas of Indonesia}

The fossil molluscan assemblages of the Miyazaki group show the close affinity to those of the Indonesian Neogene faunas, having many turrids, olivids and being scanty of the pectinids, though the assemblages of the latter are distinguished from the former by abundant conids, strombids, and batillariids. This faunal affinity makes the correlation of the strata rather practical.

The stratigraphical standard in Java was, as is well known, established in 1913 by K. MARTIN (161-173), who in that occasion employed two methods for the determination of the geological age. The one is the percentage method in which he considered that the tropic fossil faunas contain the living species by higher percentage than the simultaneous fossil faunas of the temperate region because of the high survival ratio favoured by the ecologic conditions. The bio-chronologic standard established by MARTIN shows that the percentage of the living species contained in the tropic fauna are 50 to 60,25 to 45 , and 10 to 20 in Pliocene, Late Miocene, and Early Miocene, respectively. The other method he employed is the comparison of the faunas with other chronologically known faunas for which the particular elements were specially investigated. The biostratigraphical standard established by MARTiN for the Indonesian Neogene is: as following: 
Pliocene

Sonde beds $\quad 54$ percent living (Gastropoda)

Tjandi beds 41 percent living (Gastropoda)

Late Miocene

Tjilanang beds 30 percent living (Invertebrates)

Limestone of Liotjitjangkang 32 percent* living

Burdigalian

Njalindung beds $21\left(18^{*}\right)$ percent living (Gastropoda)

Coral limestone of Tegalsari (Nephrolepidina and Miogypsina)

Aquitanian

Riff limestone of West-Progo mountains 8 percent* living (Nephrolepidina, small Eulepidina, Cycloclypeus)

Limestone of Radjamandala (large Eulepidina)

Rembang beds 15 percent living (Mollusca; large Eulepidina, Cycloclypeus)

The species in common between the Indonesian Neogene formations and the Miyazaki group and those close allied between both the areas are listd below. In this list the specific names, geologic range in Indonesia, and the stratigraphic horizons in the Miyazaki group are indicated in the first, second, and third column respectively.

$$
\text { common species }
$$

Anadara (Scapharca) rhombea (BORN)

Dentalium hexagonum Gould

Plio. - Rec. 1st- $a$

Tonna costatum (DESHAYES)

Plio. - Rec. 4th-6th

Oliva (Oliva) ispidula LINNÉ

Xenophora (Tugurium) dunkeri MARTiN

Plio. - Rec. 2nd

Murex (Murex) djarianensis MARTIN

Plio. - Rec. 2nd

$M$. (M.) verbecki MARTIN

Plio.

1 st- $a-6$ th

Clavatula djocdjocartae serrana FISCHER

Plio.

2nd-4th

Terebra insulindae FISCHER

Plio. $\quad 3$ rd

Plio. $\quad 4$ th

Celementia (Clementia) papyracea (GRAY)

Plio. 6th

Uberella rufa (BORN)

Apollon bitubercularis (LAMARCK)

Gemmula granosa (HELBLING)

Low. Mio. - Rec. 1st-6th

Cucullaea pamotanensis MARTIN

Low. Mio. - Rec. 4th

Low. Mio. - Rec. 4th

Low. Mio. - Rec. 6th

Low. Mio. 1st-a

Allied species

Paphia (Paphia) rimosa (PHILIPPI)

The species indicated in the brackets are the corresponding allies from the Miyazaki group

$[P .(P$.$) exilis exilis Shuto]$

Plio.

1 st

Uberella gendingensis (MARTIN)

[U. yokoyamai KURodA \& HABE] Plio.

4th-5th

* Revised figure by WANNER and HAHN (1935). 
Granulifusus rufinodis timorensis (FISCHER)

[G. matsumotoi SHUTO]

Plio.

4th-6th

Ancilla (Baryspira) junghuhni (MARTIN)

[A. (B.) oyamai SHUTo]

Plio.

4th-6th

Trigonostoma crispata (SOWERBY)

[T. kurodai MaKIYAMa]

Plio.

4 th

Bathytoma grissensis MARTIN

[B. striatotuberculata (Yokoyama)] Plio.

4th-6th

Orthosurcula gendingensis MARTIN

[O. pervirgo (YOKOYAMA)]

Plio.

5 th

Clavatula ktolemandoënsis (MARTIN)

[C. astuta (YoKoYAMA)]

Plio.

4 th

Terebra javana MARTIN

[T. reticulata YoкоYама]

Plio.

4th-6th

Solecurtes pectiniferus MARTIN

[S. divaricatus (LischKE) subsp.]

Late Mio.

1 st- $a$

Lyria edwardsi D'ARCHIAC

[L. mizuhonica MAKIYAMA]

Late Mio.

4th-6th

Surculites carinata MARTIN

[S. siogamensis NomURA]

Late Mio.

1 st- $\beta$

Crassatellites (Crassatellites) parva MARTIN

[C. (C.) takanabensis SHUTo]

Vasticardium njalindungense (MARTIN)

[V. hyugaense SHUTO]

Late Mio.

6th

Amussiopecten singkirensis (MARTIN)

[A. iitomiensis (OTUKA)]

Early Mio. $\quad$ 1st- $a$

Early Mio. 1st-2nd

From all the available references the faunas of the Miyazaki group are concluded to be related rather closely to the Indonesian Neogene faunas than to those of northeast and northwest Japan. The Miyazaki district must have been included within the Indo-West Pacific palaeobiogeographical province (DAviEs, 1934, 18; EKMAN, 1953, 11-29) together with the Pacific side of southwest Japan in the time of the Medial Miocene to Early Pliocene. Accordingly the Neogene faunas of south Japan can be practically correlated with those of the Indonesean Neogene, if the minute and very exact conclusion is not claimed.

As indicated in the preceding list, the fourth, fifth, and sixth horizons, i.e. the Amusiopecten praesignis-Granulifusus dualis zonule of the Miyazaki group contain many Indonesian Pliocene elements and their allies and can be correlated with the Javan Pliocene. The first and second horizon of the former, the Paphia grata tsumaensis-Granulifusus koyuanus zonule and the Kaneharaia kaneharaia-Trachycardium shiobarense zonule, having the important Indonesian Miocene indices, can be correlated with Javan early Late Miocene. The evidence from the percentages of the living species to the total species supports this conclusion. That is to say, the sixth horizon (56 percent) and the second one (40 percent) correspond with the Sonde (54 percent) and the Tjandi (41 percent) beds respectively, while the first- $\beta$ (35 percent) and 
first- $a$ (28 percent) horizons correspond with the Tjilanang beds (32 percent). Considering the above data I am inclined to correlate the sixth horizon of the Miyazaki group with Javan Sondian, fourth to fifth with the Cheribonian, second and third with the Tjandi, and the first with the Tjilanang of Java.

(9) - Correlation with European and American standards

Correlation of the Tertiary, especially of the Neogene molluscan faunas of south Europe and east Asia is very difficult as pointed out by many authors because the two regions belong to different palaeobiogeographic provinces, the Mediterranean and the Indo-West Pacific. About 20 percent of the genera of the Miyazaki group including many important time indicators are quite indigenous to the west Pacific province. Consequently no reliable result could be expected from the comparison of the mollusks of the Miyazaki group to the European Neogene faunas. However, the fauna of Indo-West-Pacific province is more closely allied with Mediterranean fauna than it is to the faunas of other separated provinces such as northwest America or Atlantic, but the common species between the Mediterranean and the Indo-West-Pacific province are very few. This must hav been caused by the Middle East barrier, which separated two provinces almost throughout the Neogene time except for the first and possibly the second Mediterranean transgressive stage. Moreover many of the species in common between both provinces unfortunately show very wide geologic ranges, and accordingly they are not available for the correlation on a fine scale.

Certain index genera, i.e. Amussiopecten, Palliolum, Megacardita, Orectospira (="Trochocerithium") etc., do occur at several localities and horizons in both regions, but they are rather few in number and range generally over four stages. Consequently the correlation based on them is not necessarily fine and exact.

More accurate intercontinental correlation of the Tertiary formations is possible with the aid of the larger foraminifers and the plankton ones, as is well known. Especially the remarkable advancement in the study of the planktonic foraminifers in the last decade have enabled the world-wide correlation of the strata on a fine scale. This is probably free from the serious and unavoidable risk resulted from the inter regional migration of the organisms. On the foundation of the studies on the Caenozoic plankton foraminifers of Middle America of Bolli, LoEBlich, and TAPPEN (1957, 3-50), and Blow (1959, 1-193) established the foraminiferal zones. According to them Middle American foraminiferal zones are as follows: Catapsydrax dissimilis zones, C. stainforthi zone, Globigerinatella insueta zone, and Globorotalia fohsi zone in ascending order for the early Miocene, Globorotalia mayeri zone, G. menardii menardiiGlobigerina nepenthes zone, and Sphaeroidinella seminulina zone for the Middle and Late Mĩocene, and Globigerina bulloides zone for the Pliocene. AsANo et al. (1959) followed BLow in the study of the zonation by plankton foraminifers and presented a preliminary correlation table of the selected Japanese Neogene formations from which a necessary part is quoted here in the following para- 
graphs. According to them the lower part of the Kakegawa group ranges from the upper half of the Globorotalia menardii menardii-Globigerina nepenthes zone to the Sphaeroidinella seminulina zone. The Sagara group, which underlies the Kakegawa group, represents the Globorotalia fohsi zone and the G. mayeri zone. The Saigo group, which underlies the Sagara group, represents the Globigerinatella insueta zone.

The lower half of the Miyazaki group below the fourth fossil horizon is characterized by the plankton foraminifer such as Orbulina universa, Globorotalia fohsi fohsi, G. fohsi lobata, and Sphaeroidinella rutschi. While the upper half, the fourth and the higher horizons, is featured by Orbulina universa and Globorotalia menardii. Comparing with BLow's scheme of the foraminiferal zones, the former may be correlated with the Globorotalia fohsi zone to the G. meyeri zone and the latter with the G. menardii menardii-Globigerina nepenthes zone. In other words the former is Late "Burdigalian" to "Vindobonian" and the latter is "Sarmatian" in age, though the relation between the BLow's foraminifera zones in Middle America and the standard stages in Europe is not necessarily clear, and accordingly it may not be appropriate to use here the stage names such as Burdigalian, Vindobonian, etc.

W. H. Akers and C. W. Drooger $(1957,656-678)$ put in order the various data on the Oligocene and Miocene biostratigraphy of Gulf region. According to them the European type Aquitanian and Burdigalian are biochronologically represented by the biozones of Miogypsina gunteri-M. tani and by those of $M$. irregularis $-M$. intermedia respectively. These biozones are also recognized in the Gulf region. European Helvetian is featured by $M$. cushmani and the Gulf Coast Helvetian is represented by the biozones of $M$. cushmani-M. mexicana. Consequently Middle American Helvetian in this sense is quite correlated to the type Helvetian. The Middle American Helvetian, thus established, contains in its upper part the distinct assemblage of plankton foraminifers characterized by Globigerinatella insueta, Globigerinoides bispherica, and Globorotalia praemenardii. While the overlying Tortonian is featured by the dominant occurrence of Globorotalia fohsi. Orbulina universa and Globorotalia menardii appeared together with the earliest representative of $G$. fohsi group. As regards to the zonation by plankton foraminifers these results do not differ essentially from BLow's, but are quite divergent from BLow's in the reference to the international stages. If AKER's correlation is valid, it is better to correlate the lower half of the Miyazaki group, Megacardita oyamai zonule and Paphia grata tsumaensis-Granulifusus koyuanus zonule, to Tortonian. This conclusion is rather harmonious with the result of the correlation of the Miyazaki group to the Indonesian Neogene on the basis of the mollusks and also of the larger foraminifers to be mentioned below.

Among the larger foraminifers only Operculina is known from the Miyazaki group. Operculina complanata japonica HANZAWA, O. ammonoides (GRoNoviUs), and $O$. bartschi ornata (CUSHMAN) respectively occur in the basal sandstone, the silty beds between the second and fourth horizon, and the sandstones of the 
uppermost horizon. In our country O. complanata japonica frequently occurs in the Miogypsina bearing beds and also in a little higher part. In other words $O$. complanata japonica survived slightly later than Miogypsina did. In the case of the Miyazaki group O. complanata japonica horizon is not in the Miogypsina zone, but represents a little higher horizon, because the accompanying fossil assemblage of the basal Miyazaki group contains of younger elements than that of the Miogypsina zone. The subgenus Miogypsina is said to range generally from basal Miocene to middle Miocene. If this is generally accepted, the basal bed-Operculina complanata japonica horizon-of the Miyazaki group* should be just above the middle Miocene. This conclusion quite agrees with the correlation of that group to the Indonesian Neogene by molluscan fossils as mentioned in the foregoing section.

As already noted the molluscan faunas of the Miyazaki group are quite dissimilar to the Mediterranean faunas with only a few allied elements. Amussiopecten, Megacardita, and Orectospira are the typical Mediterranean-IndoWest-Pacific elements. Amussiopecten ranges from Aquitanian to Tortonian in the Mediterranean region and also occurs in the so-called Miocene formations of the southeast Asia. Japanese species A. iitomiensis (OTUKA) [=A. hyugaensis Shuto] and A. praesignis (YokoYAma) are closely allied respectively to A. passinii (MENEGHINI) [=A. flavellum UgOLINI] and to A. burdigalensis (LAMARCK) of which the former occurs in the Helvetian of Sardaigne and the latter in the Aquitanian and the Burdigalian. Megacardita appeared in the Burdigalian beds of the Italy. While our Venericardia (Megacardita) megacostata SHuto is closely allied to $V$. (M.) jouanneti BASTELOT, which is the earliest species of this subgenus. In Europe "Trochocerithium" has not been known from any formation except for the Miocene. If the geologic ranges of the Japanese allies were same as those of the European genera and species, in other words, if the time needed for the regional migration of these forms was negligibly short and if the off-springs in both regions survived until almost the same age, the lower half of the Miyazaki group might roughly be correlated to Burdigalian and Vindobonian of Europe. This conclusion is consistent with the result of the correlation by plankton foraminifers under the BLow's scheme, but disagrees to the results of the correlation to Indonesian Neogene by means of larger foraminifers and mollusks and also to the those by plankton foraminifers in AKERS' scheme.

As indicated in the preceding statements the results of the correlation are remarkably divergent according to the different groups of organisms or to the different stratigraphic scheme on which different authors are depending. However the results from the comparison of the molluscan assemblages, index larger foraminifers, and the plankton foraminifers in the scheme of AKERS et al. are rather harmonizing. Hereupon I am inclined to come to the conclusion for the chronology of the Miyazaki group that the Paphia takanabensis subzonule is

\footnotetext{
* Below the Operculina bed the siltsones and sandstones of hundreds meters are developed at the southern extremity.
} 
Table 3. Correlation chart of the selected Neogene formations.

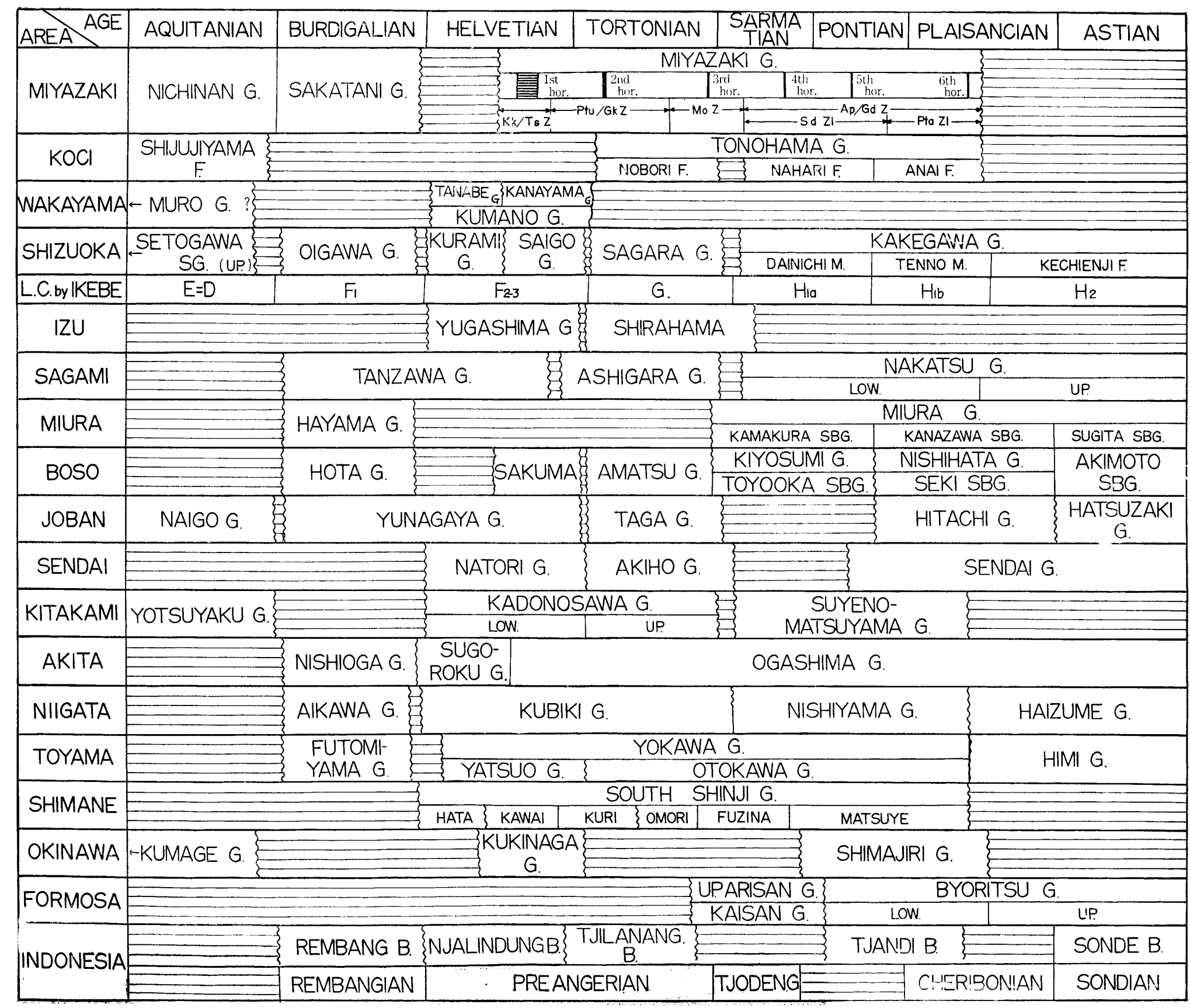


Lower Pliocene (Plaisancian), Siphonalia declivis declivis subzonule is Pontian, Megacardita oyamai zonule is Sarmatian, Paphia grata tsumaensis-Granulifusus koyuanus zonule is Tortonian, and Kaneharaia kaneharai-Trachycardium shiobarense zonule is Late Helvetian in age. Results of the correlation with various faunas are summarized in table 3 .

\section{Speciation of some molluscan species}

Concerning the evolution of the mollusca, many authors have hitherto offered piles of data, successful considerations, and profound theories.

Evolution is originally a many-sided subject, because it is realized in nature under the controle of the compound function of numberless factors, both biotic and inorganic. However, if simplification is allowed, the evolution theory may be classed into following two major branches on the basis of the objective material which the researchers deal with. One is the palaeontological evolution theory based on the fossil materials and the other is genetical one based on the heredity of living organisms.

The palaeontological evolution theories are the assumption constructed upon the data, on one hand, of the morphology, comparative anatomy, and ontogeny of the given fossils, which are the sole records of the ancient organisms, and on the other hand of the stratigraphic horizons of the beds containing the fossils. The proposed phylogeny of Umboniinae (MakiYama, 1924, 119-130 and 1935, 241-255; OYAMA, 1955, 169-176; Shuto, 1956, 47-66; Sugryama, 1935, 404-430; SuZUKI, 1934, 67-81 and 537-539) and Turritellidae (IDA, 1952, 1-64; KoTAKA, 1959, 1-135; MARWICK, 1957, 1-55; MERRIAM, 1941, 1-214) is the famous example of this category based on the molluscan material. In spite of the glorious success in the assumption of the genealogical tree, it betrays the fatal deficiency in the consideration of the speciation process.

Whereas the genetical school of the evolution theory has tried to clarify the evolution phenomena with the aid of the results of the experimental genetics, which follows up an inquiry of the mechanics of the gene flow in the organic system. In this school there are every indications that absoluteness of the genes has been sustained in an unsparning manner, and in consequence the theory, say, was often push against the biased view as regards to heredity mechanism. One of the most valuable contributions of the researchers of this school to the evolution theory is the appropriate estimation of the variability of the species for the basis of the speciation mechanics.

Both of these two branches of the evolution theory have greatly advanced recently. Palaeontological evolution theory, on one hand, has become more minute and accurate with the aid of the knowledge of the statistics, ecology, and even biochemistry. On the other hand, genetical evolution theory also has put an entirely new aspect since the introduction of the knowledge of the modern population genetics and cell-genetics. Many researchers have made every effort to organize these divergent branches into a harmonious complete theory. In spite of the partial success, the complete organization has not been realized yet. 
Actually it seems that the vast difference in time interval, which the genetics: and the palaeontology concern with respectively, derives the essentially divergent concept on time and forms an incombatible barrier between the two branches.

The actors of the evolution phenomena in nature are originally the interbreeding populations, the largest reacting units in nature. We should put in mind that even the most rapid progresses in evolution phenomena were realized through the action and reaction of the interbreeding populations, though some genera (or larger categories) seem to have been off-sprung directly and immediately from other genus (or larger category) in such rush evolution. Accordingly the analytic investigation of the interbreeding populations is also necessary for the consideration of the evolution of the ancient organisms, though difficult in practice. This concerns naturally with the variability of the populations, degree of isolation among the populations, and with the isolation mechanics. This seems to be one way through which the respective knowledge of the neontology and palaeontology can be united together into the real evolution theory. Furthermore the latest progress in biochemistry informs us that any change in the characters of organization is realized through the metabolism and that the mechanics of the metabolism in organisms is in a close connection with the environmental factors.

Taking these advanced knowledges into consideration I am inclined to think that it is most effective for the comprehension of the meaning of the variation of some organisms in time and space (geologic and geographic variations) to synthesize the variation phenomena in the organisms and the fluctuation in the environmental factors. In the case of the fossil materials the morphologic variation in the autochthonous intraspecies population should be examined in connection with the physico-chemical characters of the enclosing bed, which at least is the reflex of a part, if not all, of the palaeo-environmental factors. In brief the fossil materials themselves alone are meaningless for the comprehension of the essence of the evolution without any knowledge of the enclosing sediments.

The distinction of the interbreeding population in the fossil material from any locality or area of a given horizon can be made out through the statistical analysis of the morphologic characters. The biological statistics teaches us that the distribution of a appropriately selected form character of the given fossil group may be one modal, if whole individuals belong to a perfectly fused population. If the frequency distribution curve of any one character is bimodal with the peaks separated at the base, it is naturally considered that the material consists of utterly different two interspecies populations. In the case the curve is bimodal but the peaks are separated only by a shallow valley, the situation is not simple. In this case this fossil group apparently includes more or less isolated populations. Such a phenomena as above noted may be seen both in the inter- and intraspecies populations, if only one character is treated. However the statistical discrimination in the same manner of various characters may reveal the correct order of isolation between or among the populations of given fossil material, and proves the validity of this method for the criterion to set the 
population at a real taxonomic level. In the case that one or more characters show bimodal distribution, the intermediate forms in regards to the character in question should be examined. Through these treatments we may be able to keep our conclusion from a fatal error in distinguishing the interbreeding populations.

The first fossil horizon of the Miyazaki group, as already mentioned, extends laterally beyond the boundary of the mega-facies. The fossil beds themselves of this horizon are differentiated into a few seggregations with particular sedimentary and biological features. Generally the seggregated areas are characterized by the combination of the stenotopic species. However many eurytopic species occur over several areas without any apparent morphologic difference throughout the entire areas. It is very interesting that, on one hand, some of the eurytopic species illustrate the microgeographic variation and the isolates differ slightly from one another in the mean values of the measureable characters, though within a single area these intraspecies populations are almost homogenous. On the other hand some populations, slightly divergent but not so different as to be separated into the particular species, occur within a single area and even at a single locality. According to MAYER's definition the phenomena demonstrated by the former group is allopatry and the latter sympatry.

\section{I-Allopatric polytypy}

The fossil beds of the first horizon yield commonly the following species, each of which consists of a few allopatric intraspecies populations. They are Clementia (Clementia) papyracea (GRAY), Venus (Ventricoloidea) foveolata miyazakiensis (Shuto), Joannisiella cumingii (HANLEY), Paphia (Paphia) hirabayashii OTUKA, and Eudolium oyamai SHUTo. The description of the allopatric populations of these species are given below.

\section{(1) Clementia (Clementia) papyracea (GRAY)}

The specimens of Clementia from the first horizon are readily distinguished from $C$. (C.) vatheletii MABILLE in having smaller and thinner shell with stronger concentric rugae. The majority of the specimens is preserved in the silty sandstones with the valves conjoined in spite of the fragility of the shells. This may due largely to the life form, burrowing habit of this species. Many of the specimens are unfortunately more or less deformed and this makes difficult to examine the exact morphologic variation. Living forms of this species dwell on the mud or fine sand bottom of the protected shallow water in the region from Formosa to Australia.

In general there is not any significant difference in the shell-outline and ornamentation among the populations of minor areas within the Miyazaki district. The apparent difference among them are observed in the size of the shell, which is represented by the length (L) for convenience, and the convexity of the valve represented by the ratio of the depth to the length $(\mathrm{D} / \mathrm{L})$. Either of these measures is the maximum in the specimens of the Tano area, especially of Hôkôbô, where the species in question has the densest population making 
itself as the dominant element of the molluscan assemblage, and is smaller in those from the thinly populated areas. It is a remarkable fact that the size and convexity of the valve of the constituent of the population generally corresponds with the size of the population itself. This means that the shallow muddy flat of the Tano embayment might have been most favourable habitat for this species among the serial locations and this follows from the very nature of the life form of the species above noted. Any morphologic and ontogenetic difference other than the above cited is not observed among the local populations of Clementia (Clementia) papyracea in the first horizon.

(2) Venus (Ventricoloidea) foveolata miyazakiensis (SHUTo)

Venus (Ventricoloidea) foveolata miyazakiensis (SHUTO) is closely allied to $V$. ( $V$.) foveolata cassinaeformis (Yokoyama), a Pliocene element of the Pacific side of southwest Japan, and to $V$. ( $V$.) foveolata foveolata (Sowerby), a living form in the warm waters around Formosa, Okinawa, and south Japan. $V$. (V.) f. cassinaeformis is distinguished from the present subspecies in its.

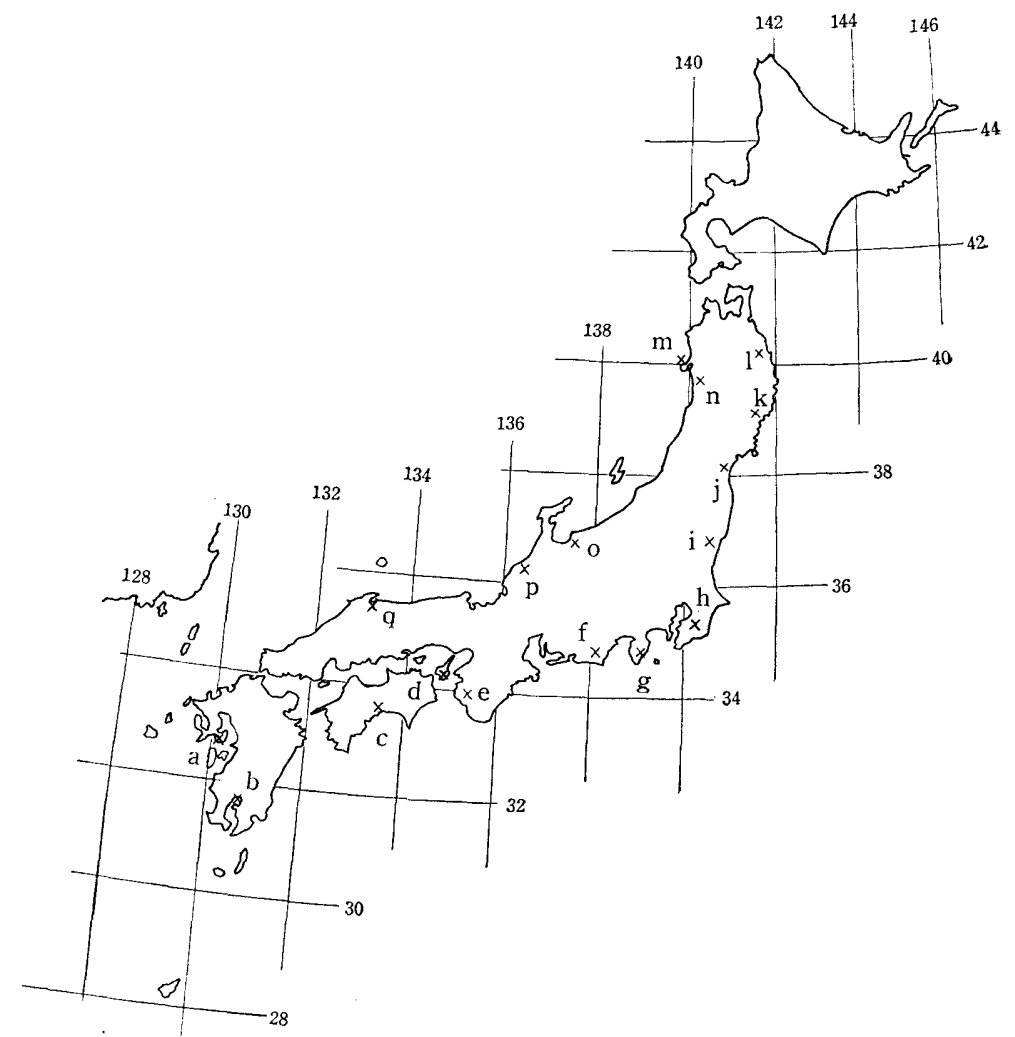

Text-fig. 14. Index map showing the referred localities.

a.... Kuchinotsu group (Low. Pleisto.); b.... Moyeshima formation (Mid. Pleisto.) ; c.... Tônohama group (Upp. Mio. -Low. Plio.) ; d....Fukura; e....Kii (Wakayama); f....Kakegawa group (Plio.); g.... Shirahama. group (Mio.-Plio.) ; h.... Bôsô ; i....Jôban ; j....Sendai ; k....Sanriku ; l....Kadonosa group (Mid. Mio.); m.... Oga; n....Akita; o... Toyama; p....Fukui ; q.... Matsuye. 
higher shell with much more circular outline and curved postero-dorsal margin. $V$. (V.) f. miyazakiensis differs from foveolata foveolata in having lower shell with more straight postero-dorsal margin. Furthermore the former has a tendency to become less convex than the latter in the adult stage, though the convexity of the valve is similar in two forms until the adolescent stage is reached in the ontogenetic growth.

$V$. (V.) $f$. miyazakiensis occurs abundantly in the first horizon of the Aya area and at Haigano of the Tano area. The adult specimens from Haigano are small with the standard size of 22 to $23 \mathrm{~mm}$. in length. While those from the Aya area are measured to be 34 to $40 \mathrm{~mm}$. in length. The height-length ratio and the depth-length ratio are 85 to 95 percent and about 24 percent in the majority of the former material and 79 to 84 percent and 20 to 22 percent in the latter group. That is to say, the shells of the specimens from Haigano are as a whole smaller, higher, and more convex than those from the Aya area. The specimens from the Aya area include four populations of four horizons and there is no significant difference in the character in question among these four populations. Another small number of specimens were collected from Oyamada of northeast part of the Yusunoki peninsula and Kagamisu pass, north side of Boroishi cape, both locality of which as already noted might have been influenced by more agitating and purer open water than the Aya and Tano areas. It is interesting that these specimens are still smaller, higher, and more convex than those from Haigano in Tano area.

\section{(3) Joannisiella cumingii (HANLEY)}

Joannisiella specimens from the first horizon are apparently related to $J$. cumingii (HANLEY), J. semiasperoides NoMURA, J. meisensis MAKIYAMA, and $J$. takeyamai OTUKA. Among these species $J$. meisensis is readily distinguished from the present specimens in having the ovoid shell with rounded postero-dorsal margin. J. takeyamai also differs from the present specimens in the moderately curved postero-dorsal margin and broadly curved anterior end.

Some authors consider $J$. semiasperoides NomURA is a synonym of $J$. cumingii (HANLEY), and others have the opinion that the two are particular and different species. The said major difference between the two is that $J$. cumingii (HANLEY) is more inflated than J. semiasperoides NomURA. However the examination of some recent and fossil specimens of this group indicates that the convexity of the valves of this group varies within a remarkably wide range ( $\mathrm{D} / \mathrm{L}=28$ to 50 percent) and there is no clear boundary between cumingii and semiasperoides. Accordingly it is not appropriate to separate this group into independent species. Joannisiella specimens from the first horizon are identified with $J$. cumingii (HANLEY) in this sense.

The shell form and the ornamentation do not significantly differ among the populations of respective areas. The form characters are most stable in the Tsuma specimens showing the narrow range of variation, and rather unstable in the Tano and Aya specimens. The major difference among these populations concerns with the dimension. The specimens from the Tsuma area are largest in 
size and least inflated. Those from the Tano and Aya area are similar in size, but the former is somewhat more inflated than the latter. Comparing these populations with other known ones, the Tsuma population resembles the living ones from the shallow bays of Fukura and Oga in the convexity of the valves and is comparable in size with those of Moyeshima Pleistocene bed, which is characterized biologically by the open water association of 100 to $200 \mathrm{~m}$. depth. 'The Aya and Tano populations resemble those from Fukura, Oga, and Kuchinotsu in size and those from Moyeshima in the convexity of the valves.

These facts suggest that $J$. cumingii (HANLEY) has the tendency to have less inflated valves of more stable form under the stronger influence of coastal water than under the influence of the open sea water. This may be natural, because the species flourishes so frequently in the restricted bay waters that it is considered as an indicator of such environment. The observed variation in morphology above mentioned may be ecological in origin.

\section{(4) Paphia (Paphia) hirabayashii OTUKA}

The specimens of this species from the first horizon of the Miyazaki group differ from the holotype from the Shizukawa group of Shizuoka Prefecture in having the valves with more produced and longer anterior parts and coarser sculptures. They contain two end forms, one of which closely approaches to the type and is distributed in the Tsuma area, and the other which has smaller and higher shells provided with closer ribs, being distributed in the Tano area. They were named $P$. $(P$.) hirabayashii koyuensis SHUto and $P .(P$.$) hirabayashii$ tanoensis SHUTo respectively (SHUTO, 1957-b, 139-160). Another population, showing the intermediate feature between these two forms, is distributed in the Aya area, the transitional area between Tsuma and Tano. As for the above mentioned circumstance these three populations seem to illustrate a cline or morphoseries. However the characteristics of the concentric ribs, which are very significant in studying the ontogeny of the species of Paphia, do not exhibit the serial change by areas in good order. The concentric ribs are closest in the specimens of the Aya population, in which the minute riblets of post-embrionic stage abruptly become large at early juvenile stage. While in the specimens of the Tano population this boundary in the development of the ribs is at the transitional stage between juvenile and adolescent and in those of the Tsuma population even the post-embrionic ribs are not so minute. These facts suggest that three populations do not form a mere ecological morphoseries or cline corresponding to the serial environments. In other words it is reasonable to assume that these microgeographical populations are isolated not merely ecologically but also genetically to some extent.

\section{(5) Eudolium oyamai SHUTo}

Eudolium oyamai, a fossil species, is featured by the alternately arranged primary and secondary spirals and by the granulated primary spirals on the upper part of the body whorls. The present species occurs in the first horizon of the Tsuma and Tano area with almost equal frequency. The specimens from 
the Tsuma area are measured $25 \mathrm{~mm}$. or less in diameter of the body whorl and have about 13 primary spirals on the body whorl. While those from the Tano area show the diameter of $15 \mathrm{~mm}$. or less and are provided with about 15 primaries. The granules on the primary spirals develop on the upper third of the body whorl in the Tsuma specimens and only on the shoulder in the Tano specimens. In other words the adult specimens from two areas differ not only in size but also remarkably in ornamentation. The further examination of the development of the spirals, however, reveals that the specimen groups of two areas quite agree in regards to the rib character at the juvenile stage and are not essentially different even at the early adolescent. The specimens of the two populations are provided with about 12 primary spirals altogether in the adolescent stage, which are coarser on the upper half of the whorls. In conclusion the two populations diverse into different branches in the course of the later stages of the ontogenetic development. Such a difference as above mentioned must be behind the controle of the purely ecological factors on the ground of the known varieties of living species, and may probably be under the genetical controle. Under the present taxonomic situation it is not objective but rather subjective to judge these differences as intraspecific or interspecific. Accordingly my disposition of these populations as the same species is not necessarily concrete, however it is indisputable evidence that these very closely allied populations diversed from a common ancestor.

As indicated in the foregoing paragraphs the intraspecies allopatric examples include at first the species illustrating merely ecological variety (ecodeme) as in Clementia papyracea (GRAY), Ventricoloidea foveolata miyazakiensis (SHUTO), Joannisiella cumingii (HANLEY); secondly the one demonstrating rather the complicate variety based on the combined effect of the ecological and genetical factors (ecogenodeme) as Paphia hirabayashii OTUKA; and thirdly the one consisting of the genetically isolated genodemes as Eudolium oyamai SHUTo. In brief the extent and the mechanics of isolation in the intraspecies allopatric forms are not the same in different species (or cases), but divergent by every degrees. This is very significant in the consideration of the speciation process of the marine benthonic mollusks in the correspondence of the environmental influence.

\section{II-Sympatric polytypy}

The occurrence of the closely allied fossil species in common at one locality readily indicates that these allies must have dwelled together in the same habitat, if the assemblage is autochthonous. It is a well known tendency that the allies expell each other within one and the same niche because of the resemblance of the life form. How is the tendency demonstrated in the benthonic molluscan world? To clarify the relation between the alliedness and the difficulty of symbiosis of any molluscan group I examined the catalogues of the molluscan assemblages from the selected 230 localities. The reports of the several authors, who have not necessarily the same concept about species, are not on the same 
level of confidence, though they are selected so as to make the least bias. The result is as following; (a) Coexistence of the different species of the same genus is rather frequent. According to my checkup the synbiosis of this category are exemplified in the assemblages of 39 percent of whole localities and in the species of 14 percent of the total element of whole assemblages. (b) Coexistence of the close allies is checked at about 20 percent of whole localities. (c) Coexistence of the intraspecific forms is exemplified at about 8 percent of the localities. The subject of this section concerns with (c) of the preceding paragraphs. Though the symbiosis of the intraspecific forms within one and the same niche is really uncommon as above mentioned, it is not so rare to unable the scientific discrimination of it.

In the Miyazaki group the intraspecific sympatry is demonstrated by Crassatellites (Crassatellites) tenuiliratus SHUTo and Paphia (Paphia) exilis SHUTO from the first horizon. The descriptions of the intraspecies populations are given in the following section.

\section{(1) Crassatellites (Crassatellites) tenuiliratus SHUTO}

The present species occurs only at Akatani and Kano of the Aya area. It is featured by its straight posterior half of the ventral margin and the unique ornamentation which consists of the fine but equally and regularly spaced lirae throughout from the umbonal to the ventral area. It contains two end forms, one of which is laterally elongated and named $C$. (C.) tenuiliratus tenuiliratus, and the other does not show the lateral elongation, but roundly triangular outline and is named C. (C.) tenuiliratus triangularis. These two forms occur together even in one and the same block, though the former is more frequent than the latter. The most direct and practical criterion to distinguish the two is the ratio of the length of the postero-dorsal margin to that of the antero-dorsal one (P-A ratio). The frequency of the $\mathrm{P}-\mathrm{A}$ ratios measured at the mixed sample shows apparently bimodal distribution. That is to say this specimen group contains two populations which are different each other in their mean values of the important characters as is suggested by the observation of the end forms. While the end forms are linked together quite continuously by several intermediate forms, which make up the complete morphoseries. Furthermore the present specimen group is very distinctive by the above mentioned diagnosis among the numberless species of Crassatellite (Crassatellites). These facts mean that the two populations were not perfectly fused into a single population prevented by some isolation mechanics, though they might have interbreeded each other in certain extent. Therefore these peculiar groups are naturally considered to be intraspecific, but not interspecific.

\section{(2) Paphia (Paphia) exilis Shuto}

The present species makes a very interesting example exhibiting the intraspecies sympatry and partially allopatry together.

It shows the wide range of variation in several characters such as the outline of the shell, the closeness of the concentric lirae, and the convexity of the valves. 


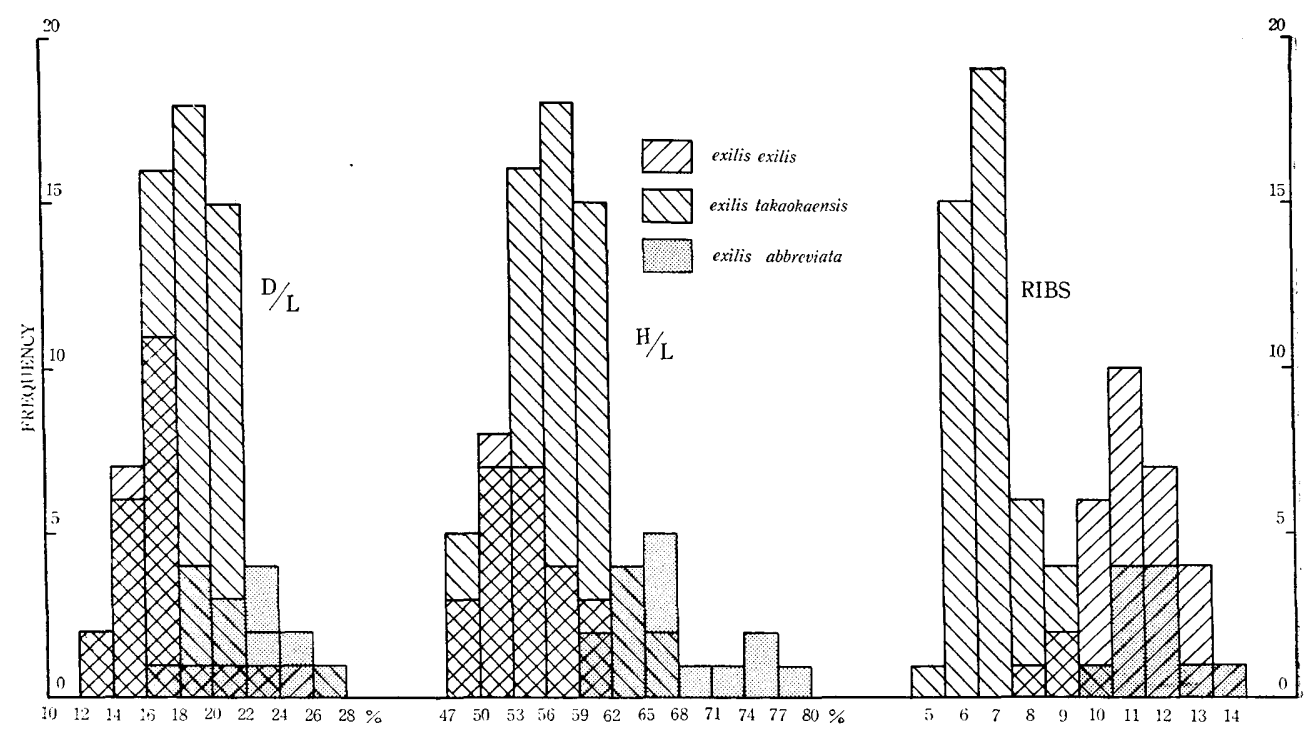

Text-fig. 15. Frequency distribution of the selected form characters of Paphia (Paphia) exilis Shuto.

$\mathrm{D} / \mathrm{L}$.... the ratio of the depth of the valve to the length.

$\mathrm{H} / \mathrm{L}$.... the ratio of the height of the valve to the length.

The number of the lirae is counted on the line of $10 \mathrm{~mm}$. from the ventral margin toward the umbo at the adolescent stage of growth, attaining about $20 \mathrm{~mm}$. long is size.

The combination of these factors realized three forms: (a) the valve is long and rather shallow; the lirae are fine and close. (b) The valve is long and rather deep; the lirae are coarse. (c) The valve is short and rather deep; the lirae are fine and close.* Consequently the distribution of the length-heightconvexity ratio (L-H-D ratio) of the valves is different by three forms in the triangle diagram and the frequency of the closeness of the lirae also does not show the one modal distribution in the mixed sample. While these forms are connected by many intermediate forms in regards to above cited characters.

These facts lead to the conclusion that on one hand (a) three forms must have interbreeded to a remarkable extent, but on the other hand (b) they must have been isolated reproductively to such a extent that they have not been fused into a single homogenous population. This assumption is also coraborated through the scrutiny of the ontogenetic development of the concentric lirae, which are the only available feature to trace the ontogeny of Paphia. As indicated in the text-figure 16, the development of the lirae of Paphia is characteristic of the respective species, at least of the respective species group. In the Japanese species of $P$. (P.) euglypta (PHILIPPI) and $P$. (P.) naganumana OTUKA

* The combination of the length of the valve and the closeness of the lirae should have resulted the fourth form, which is featured by the short valve with coarse lirae. However, in reality such form has not been found from the Miyazaki group yet. 
the central and the ventral lirae of the adult specimens are very coarse in spite of the minuteness of the umbonal ones. In the diagram of the number of the lirae protted against the distance from the umbo the curves showing the density of the lirae of these two species are not smooth, but rather bending about $15 \mathrm{~mm}$. apart from the umbo. In other words the lirae become abruptly large and

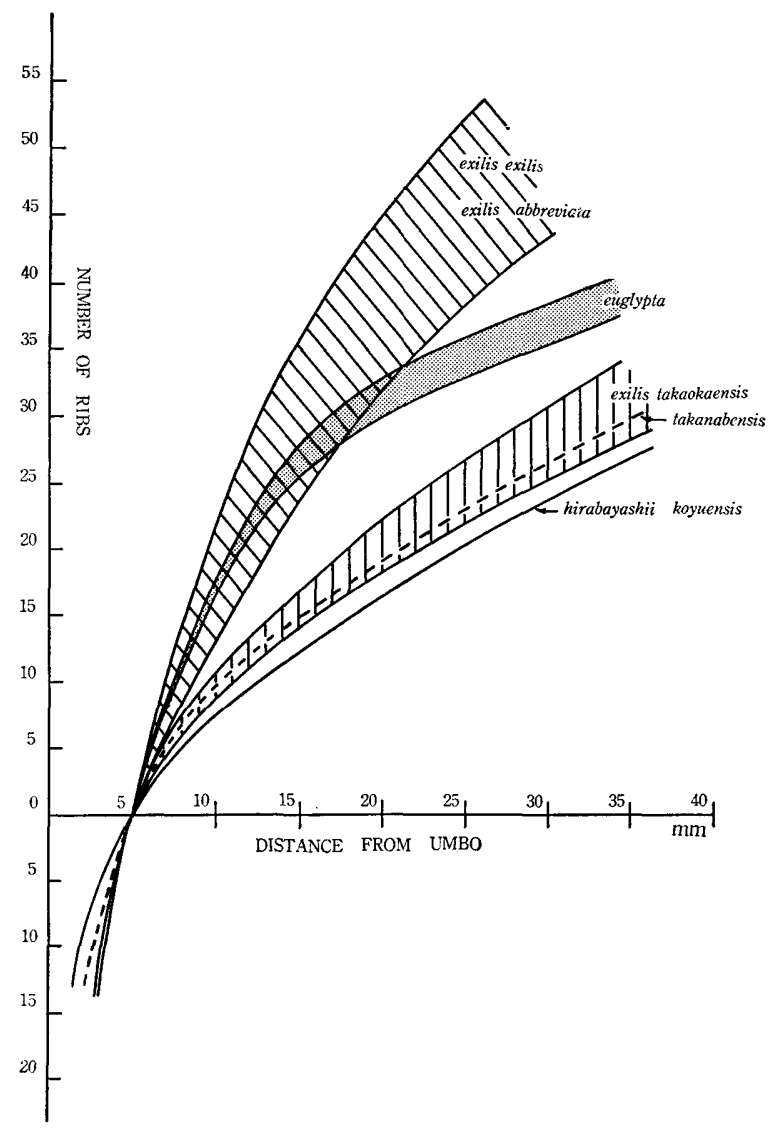

Tezt-fig. 16. Ontogenetic development of the lirae of the selected species of Paphia.

Origin is selected at $5 \mathrm{~mm}$. apart from the umbo toward the ventral margin. The reason is that the counting of the lirae of the umbonal area is often difficult because of the abrasin.

coarse at the late juvenile stage of the growth in these species. While in $P$. $(P$.) amabilis (PHILIPPI) the lirae are moderately fine at the umbonal part and become gradually large resulting the moderately coarse ones at the ventral part of the adult specimens. That is to say, there is not any abrupt change in development throughout the growth stages to result the flat and smooth curve in the diagram. Among three forms of $P .(P$.$) exilis SHUTo, P .(P$.$) exilis exilis and$ exilis abbreviata have the fine and close lirae throughout, and exilis takaokaensis 
has moderately coarse ones at the juvenile to adult stage. This results the upstanding and smooth curves of the former and the flat and smooth one of the latter in the diagram. That is to say, the development of the lirae of this group diversed into the different trends at rather early stage of the growth. This may be a reflex of the genetical difference of some extent among them.

The examination of three subspecies in the distribution and frequency in microgeographical occurrence indicates that $P .(P$.$) exilis exilis and exilis$ abbreviata are quite sympatric, and that these two and exilis takaokaensis are partially sympatric and partially allopatric. In the case of the coexistence they occur with quite different frequency. $P$. (P.) exilis exilis and exilis takaokaensis are dominant in the Aya and the Tano area respectively and the former has never been obtained from the area further south of the Tano area. While $P$. $(P$.) exilis abbreviata occurs in the Aya and Tano areas as a subordinate element.

In brief these three forms, though they are not so divergent to be separated into independent species, represent the particular populations which are adapted to the slightly different environment and may be isolated genetically from one another to some extent.

The descriptive notes on the molluscan ecology and the polytypy hitherto.

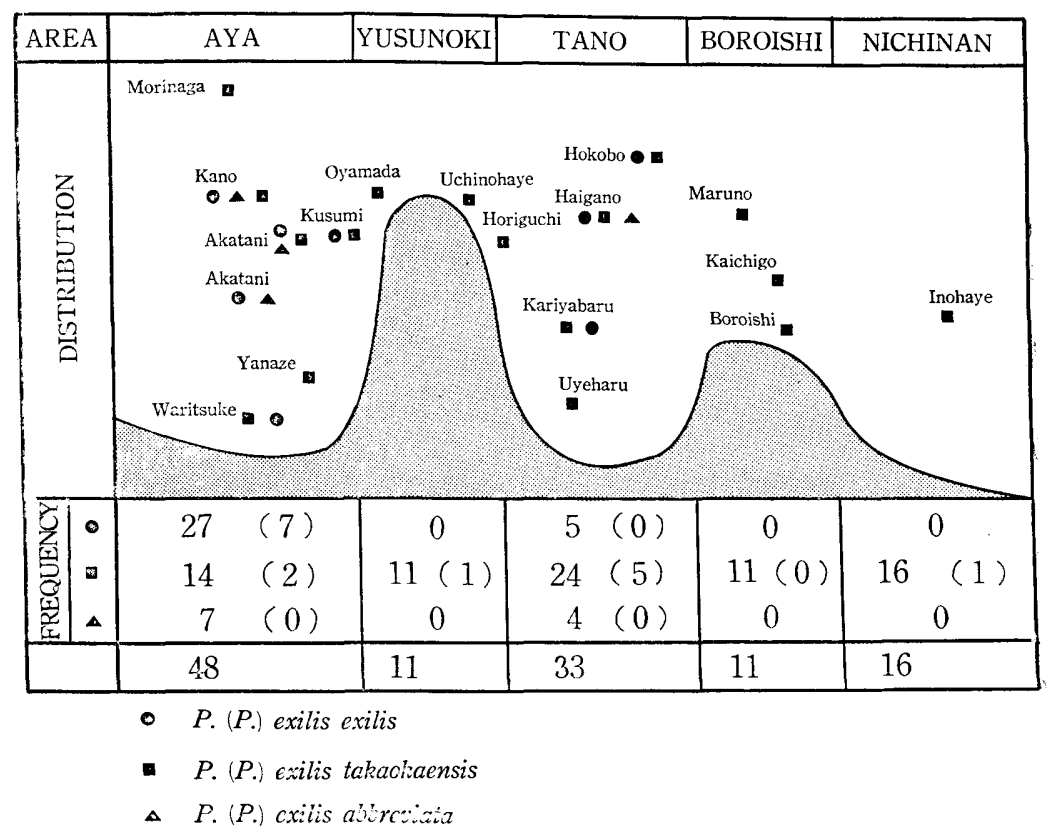

Text-fig. 17. Distribution of Paphia (Paphia) exilis SHUTo in the lower part of the Miyazaki group.

The distridution of each subspecies is illustrated in the generalized cross section, in which the grey and white ground indicate respectively the basement rocks and the Miyazaki group. Frequency is indicated by the number of the individuals obtained at each area. The figures in the: parentheses indicate the number of the conjoined valves. 
mentioned resolve themselve into four points.

(a) Several parts, ecologically differentiated and microgeographically separated, are recognized within a single fossil horizon. They are the Nangô-Nichinan, Boroishi, Tano, Yusunokihashi, Aya, and Tsuma areas, which comprise a ecoseries ranging from the condition under the purely open sea condition to that under the moderate influence of the coastal water.

(b) These ecologically characterized environments continued for considerably long duration, and the minor fluctuation did not affect the general tendency of the areas.

(c) Some stenotopic molluscan species occur in a particular area specialized by certain diagnostic environmental factors.

(d) A few eurytopic species are distributed in several areas, irrespective of the divergence of the environments, and illustrate the intraspecies allopatry. The intraspecies allopatric populations contain three groups as regards to the extent and mechanics of isolation. The first group exemplifies the mere ecodeme and the populations may not be isolated reproductively. Clementia (Clementia) papyracea (GRAY), Verus (Ventricoloidea) foveolata miyazakiensis (SHuto), and Joannisiella cumingii (HANLEY) belong to this group. The second group including Paphia (Paphia) hirabayashii OTUKA is considered to illustrate the slight genetical isolation in addition to the ecological one (ecogenodeme). In the third group, including Eudolium oyamai SHUTO, the populations are isolated reproductively to a remarkable extent (genodeme).

(e) A few populations belonging to certain species as Crassatellites (Crassatellites) tenuiliratus SHuto and Paphia (Paphia) exilis SHUTo illustrate the intraspecies sympatry and are reproductively isolated to a notable extent.

Several authors pointed out that the geographical subspecies (varieties or races of authors) of animals are closely related with the environmental factors of their habitats in general. Meanwhile we observed, as above mentioned, the surprising correspondence among the lithofacies, biofacies, and intraspecies allopatry. Herein it may be natural to suppose some relation between the physicochemical and biological environmental influence and the speciation of the organisms.

III-Environmental influence to the speciation of the marine mollusca

In this section at first the general relation between the environmental influence and the variability of the pelecypods is considered and secondly the bearing of these phenomena on the evolution of the marine mollusks, especially on the speciation, is examined. In the case of the consideration of the heredity or the speciation of any organism, we should, for the foundation, keep the plain concept of the time and space needed for the realization of the phenomena in question and should have the definite information of the physiological and ecological speciality of the populations.

(1) Time

As is generally known the rate of evolution is not the same among the 
different taxonomic units. The mammals, one of the groups showing the most rapid rate of evolution, are said to need the time of about one-third epoch for the full speciation. The evolution rate of the pelecypods must have been slower than that of the mammals.

Meanwhile the Miyazaki group attains the maximum thickness of about $2500 \mathrm{~m}$. and represents a little less than one epoch chronologically. The maximum thickness of the basal coarse beds of the group is about $400 \mathrm{~m}$. Accordingly the basal beds may approximately represent about one-sixth epoch, if the sedimentation rate is assumed as even throughout the age of the Miyazaki group. Consequently the pelecypods may have not realized the speciation cycle during the time represented by the basal bed of the Miyazaki group. Although this time interval is, thus, actually too short for the full speciation of the pelecypods, it will be sufficient enough for about a half of a million successive generations of the pelecypods, which in general need a few years for the individual maturity. The genetical meaning of a half of a million successive generations is the problem awaiting the solution.

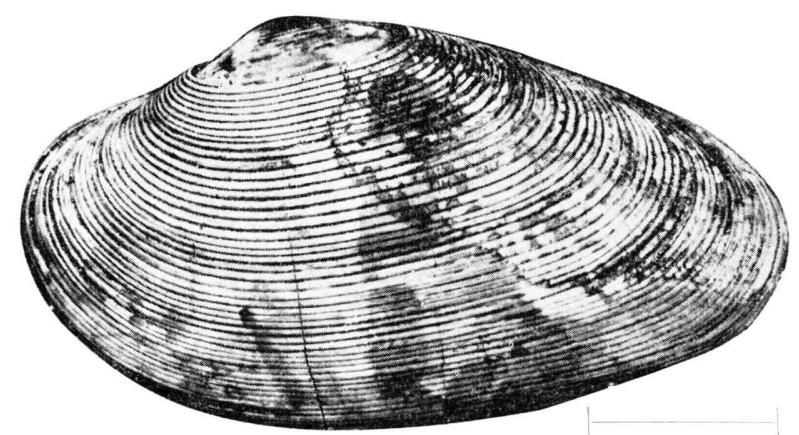

Text-fig. 18. Paphia (Paphia) takanabeensis SHUto. Unit bar indicates three $\mathrm{cm}$.

\section{(2) Space}

Comparing with the distribution area of the living species of pelecypods, the area occupied by the first horizon of the Miyazaki group is very small and narrow. Here a critical question arises whether or not the speciation phenomena observed in such a small area are equivalent to or comparable with the phenomena realized in more extensive area. This question may be offered in other expression. May such a small area contain enough range of the environments within its extent as wide as those contained in the entire occupation area of a species to provide for the enough room for the speciation of that species? Is it acceptable, if this is the case, that the speciation phenomena observed in such a small area are comparable to those realized in the entire occupation irrespective of the relative position (center, margin, etc.) of the area in the geographical distribution of the species?

Strictly speaking the range of the environmental conditions of the first horizon is at most equal to or narrower than that of the whole habitable areas of the species. However there may be practically no essential difference between the 


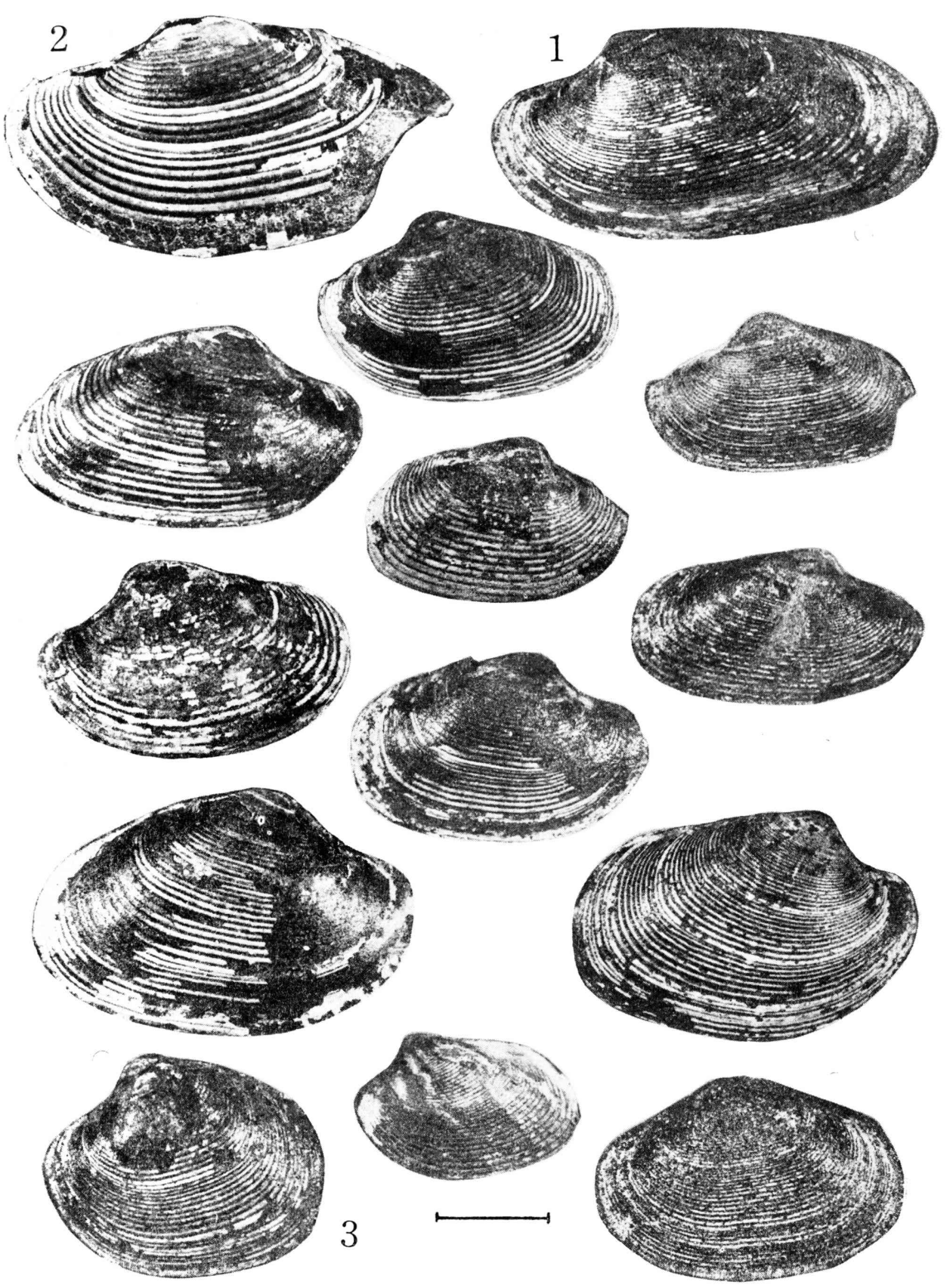

Text-fig. 19. Variation in the general outline of Paphia (Paphia) exilis SHUto.

1....P. (P.) exilis exilis Shuto, 2...P. (P.) exilis takaokaensis Shuto, 3....P. (P.) exilis abbreviata SHUTo.

Unit bar indicates one $\mathrm{cm}$. 
two so far as the present case is concerned. The reasons are as follows.

(a) Salinity: The first horizon and the immediately upper and lower sequence in the Miyazaki group contain a serial facies including sediments from purely open water to deltaic. This means the salinity range was wide enough for the ordinary marine pelecypods.

(b) Water temperature: The water temperature at the time of the first horizon is considered as high as that of the marginal area of the present-day tropic waters on the basis of the fossil assemblages and the neritic sediments cemented with highly calcareous matrix. The water temperature of the recent tropic region of west Pacific is remarkably stable and this offers the basis of the supposition of the ancient condition.

(c) Depth and bottom material: The first horizon, representing fully the neritic depth range and having the every detrital sediments, is almost quite equivalent to the recent neritic sediments except for the purely carbonaceous ones.

By all accounts the environmental conditions, at least the physico-chemical conditions, of the first horizon are almost equivalent to those of the total distribution area of the tropic species of pelecypods. Consequently the first horizon might have potentially contained all the major variants of each tropic or subtropic species on account of the dispersion at the velliger stage. Accordingly the intraspecies polytypy and the speciation phenomena of any species observed in the first horizon may not be so far from the total phenomena in whole the occupation of this species.

(3) Physiology and embryology

The limits of tolerance of the pelecypods and gastropods in regards to the environmental conditions are not constant throughout the growth stages. The limits for the development of eggs and velliger larvae are much more restricted than those for the adult life, largely because the biochemical balance tends to be broken down in the early stage of development. For example the eggs may be easily put to death by puncture or shrinkage caused by the change in osmoic pressure which corresponds with the salinity change. It is also well known that the larvae of the oyster perish altogether in extraordinary cold summer. The eggs and larvae are both stenothermal and stenohaline. In other words the marine gastropods and pelecypods are affected effectively by the environmental factors in the early stages of the ontogenetic growth. Accordingly for the geographical distribution of any benthonic molluscan species the limit of the tolerance of the larvae to the environmental influence must be carefully considered.

The examples of the first horizon, as already mentioned, show the tendency that in every polytypic species the shells are large in the specimens from the area with moderately dense population and more convex in those from the area under the stronger influence of the open water or of the agitating water than those from the areas under weaker one. These facts do agree to the general tendency observed in the living forms. It is generally accepted by the ecologists that the ontogenetic development of the marine pelecypods is accelerated in the favourable salinity, and the shells become more convex and more solid in the water strongly 


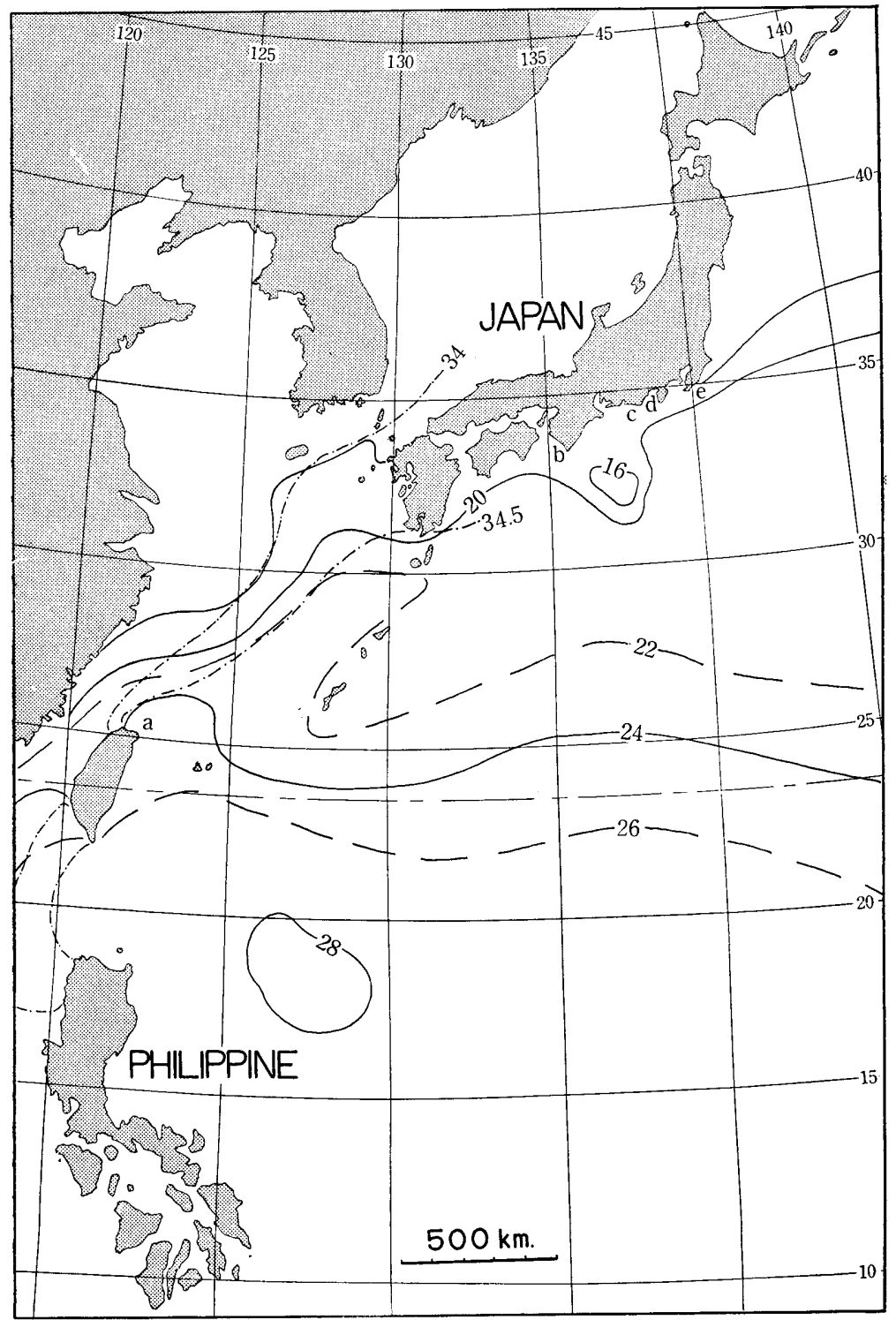

Text-fig. 20. Map of the Philippine Sea and the adjacent waters, showing the oceanographical condition in summer.

full and broken lines....isothermal lines at $100 \mathrm{~m}$. depth ; dot and dash lines.... isohaline.

a.... Kiirun; b.... Sea of Wakayama (Kii) ; c.... Sea of Enshu (Shizuoka); d....Suruga Bay; e....Tateyama Bay.

agitating than in the calm water, especially they do so in the case of the favourably high temperature. These two factors, salinity and agitation of the water are the fundamental factors to prescribe the degree of embayment.

To clarify the mutual relation among the intraspecies populations of Paphia 
(Paphia) exilis SHUTo further examination will be given in the ecological field.

IV-Paphia (Paphia) exilis SHuto group and its characters

(1) Paphia (Paphia) exilis SHuto and its close allies

$P$. ( $P$.) exilis Shuto is unique and distinctive in four characteristic features among the known species of Paphia, both living and fossil.

(a) It has the laterally elongated valves, the height-length ratio of which is 53 to 56 percent in most specimens of this group except in $P$. (P.) exilis abbreviata. (b) Its ventral and postero-dorsal margins are long and rather straight, and even in $P$. exilis abbreviata the straight part of the ventral margin is relatively long.

(c) The posterior end of the valve is obliquely subtruncated.

(d) The surface of the valve is ornamented by the high and flat-topped close concentric lirae separated by the U-shaped interspaces throughout.

There are a few known species which are provided with these characteristics altogether. Following is the brief notes on these species.

Paphia (Paphia) papilionacea (LAMARCK), living in Madagascar, Ceylon, and New Guinea. This closely resembles $P$. (P.) exilis exilis in general contour, ornamentation, and size, but the former has the valves with slightly closer lirae and a more broadly rounded postero-dorsal margin.

$P$. (P.) amabilis (PHILIPPI), living in Australia and south Japan and also occurs as fossils in the Pliocene beds of Japan and Formosa. This species resembles $P$. (P.) exilis takaokaensis in the general contour and especially in the ribbing, but has a somewhat higher shell with a smaller umbonal angle than the latter.

$P$. (P.) euglypta (PHILIPPI), living in south Japan. This species quite agrees with $P$. ( $P$.) exilis exilis and exilis takaokaensis in the outline of the shell, but has larger shell with unique ribbing than the latter two. As has been already mentioned, it is provided with very fine lirae in the juvenile and extremely coarse ones in the adolescent and adult stage.

$P$. (P.) naganumana OTUkA, Pleistocene species of the Kwanto district of Japan. This species looks like $P$. $(P$.) exilis takaokaensis, but is much larger in size than the latter and its ribbing is quite different from that of the latter like as $P$. (P.) euglypta.

$P$. (P.) exarata (PhilipPI), living in south Japan and in the Chinese seas. This species is featured by somewhat short valve and closely resembles $P$. ( $P$.) exilis abbreviata, but its valve is more convex than the latter. Furthermore the form characters are rather variable in the latter, while they are remarkably stable in the former.

$P$. (P.) lirata (PHILIPPI), living in Philippines and Formosa, and also occurs as fossils in the Pliocene beds of the same areas. This resembles $P$. $(P$.) exilis takaokaensis in general character, but has shorter valve featured by the more rounded postero-dorsal margin and rather round-topped lirae than the latter.

$P$. (P.) takanabensis ShuTo, fossil species from the uppermost part of the Miyazaki group. This species quite agrees with $P$. (P.) exilis takaokaensis in 
every character except in the size. The former is about three times as large as the latter.

$P$. (P.) rimosa (PHILIPPI), living in Indonesian waters and also occurs as fossils in the Pliocene beds of the same area. This species is allied to $P$. (P.) exilis exilis of the Tano area, but its valve is more rounded at the ventral margin and hardly truncated at the posterior end.

$P$. (P.) protolirata (NoETLING), Miocene species of Burma. This is closely allied to $P$. ( $P$.) exilis takaokaensis, but is smaller in size and provided with the smaller posterior part than the latter.

The subgenus Paphia is a typical element of the Indo-West-Pacific fauna. The living individuals of this species are obtained from the silty sand and fine sand bottom of euneritic to mesoneritic fascia of the open sea of this region. In the same region the fossil ones also occur in the sediments of similar lithology with the association of the upper and middle neritic faunas of the open water. In brief the habitat of Paphia (Paphia) exilis group, both living and fossils, is the fine sandy bottom of the euneritic to mesoneritic fascia in the tropic to subtropic waters of southeast Asia. In this region the salinity ranges from 34 percent at the highest to 31 percent at the lowest. The temperature of the surface layer of the oceanic water ranges 27 to $28^{\circ} \mathrm{C}$. at the equatorial and 20 to $28^{\circ} \mathrm{C}$. at the north end of the region. That is to say the habitat of Paphia (Paphia) exilis group is very stable in regards to the salinity and temperature.

(2) Distribution of Paphia (Paphia) exilis in the first horizon

The distribution and the frequency of $P$. $(P$.$) exilis is summarized in text-$ fig. 17.

In the Aya area the specimens of $P$. (P.) exilis exilis and $P .(P$.$) exilis$ takaokaensis obtained from the calcareous sandstone of the lowest part of the first horizon are altogether somewhat smaller than the standard form including holotype. Furthermore the former subspecies is rare in occurrence and has less convex valves provided with the coarser lirae than the standard form. While the latter subspecies occurs frequently and its form characters are rather invariable, showing the stable L-H-D ratio. Towards the upper horizon $P$. ( $P$.) exili: exilis gradually increases its population, but $P$. $(P$.) exilis takaokaensis, on the contrary, abruptly decreases with the restricted occurrence chiefly to the margin $x 1$ and rather coarse facies. In addition to the change in the size of populations the form characters also changes in these subspecies in the lapse of time. That is to say during this time interval $P$. $(P$.) exilis exilis shows the apparent tendency to become larger, and more elongate laterally, and to have more numerous lirae While in $P$. ( $P$.) exilis takaokaensis the L-H-D ratio became variable within the wide range and the convexity weakened.

In the Tano area the relatively large specimens of $P .(P$.$) exilis takaokaensis$ occurs in abundance not only in the coarse marginal facies but also in the fine central facies throughout, but $P$. $(P$. $)$ exilis exilis is obtained only from the muddy central facies in a small number. The former subspecies is slightly larger, highe, 
and more convex than the standard form from the Aya area, and the latter subspecies has slightly smaller and more convex valves with coarser lirae than the standard form.

As indicated by these facts, $P$. ( $P$.) exilis group illustrates the similar tendency in morphologic variation to that demonstrated by the above mentioned intraspecies allopatric forms. That is to say, the valves of the intraspecies forms of $P$. (P.) exilis are larger in the area with moderately denser population and more convex under the stronger influence of water agitation.

The question whether or not these phenomena are the examples of the mere fluctuation may be plainly answered by the serial change of $P$. ( $P$.) exilis exilis in a particular environment in the time interval which is represented by the basal sequence of the Miyazaki group in the Aya area. In the Aya area, on one hand, towards the upper horizon its population became denser, its valve became larger and more elongate, and the lirae closer. On the other hand the environmental condition of this area, as already stated, became gradually less oceanic towards the upper affected by the coastal water (see III-D). This supports the assumption that the adaptive evolution must have proceeded in the course of this time in that area.

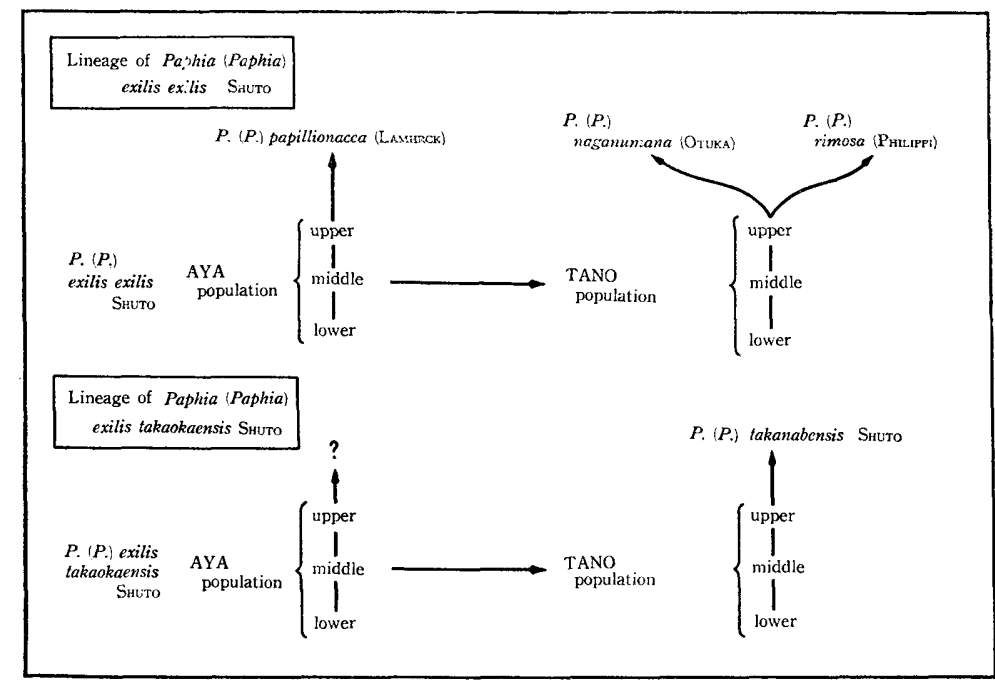

Text-fig. 21. Diagrammatic presentation of the evolution of Paphia (Paphia) exilis exilis SHUTO and $P .(P$.$) exilis takaokaensis SHUTo.$

The speciation corresponds with the differention of the environments.

$P$. (P.) exilis takaokaensis, on the contrary, is rare in occurrence and not fully characteristic in morphology in the Aya area, especially at the higher horizon. But in the Tano area, which was under somewhat stronger influence of the open water than the Aya area in almost the same age, it developed fully with typical morphology and a larger population. This illustrates that in the course of the evolution the succession of the demes become more and more 
adaptive to the environmental factors which is practically invariable in certain duration or gradually changes towards a definite direction.

\section{(3) Lineages of Paphia (Paphia) exilis SHuto group}

Because such dimensional form characters as size, convexity, L-H-D ratio. of the valves etc. much depend on the growth conditions influenced by the temporary ecological factors, the careful consideration is necessary to applyingthe results from the observation of such measurable characters to the development of other hardly measurable ones such as ornamentation. Hence I examined the evolutional lineages of $P$. $(P$.) exilis group to exclude a possible error which might be introduced into the consideration of the development of the hardly measurable character, because the recognition of the evolutionary trends of this species group may clarify the relation of it to the development of the dimensional characters.

In the preceding section I pointed out that $P$. (P.) exilis group makes up. itself a peculiar stock among the species of Paphia, living and fossil, with its unique characters. A few lineages can be distinguished within this group through orthodox palaeontological method-through the examination of the morphological alliness, stratigraphical relation of the occurrence, ontogeny, etc. Of course there are some risks in concluding the phylogenetic relationship on the basis of the resemblance in ontogenetic development, since the palingenesis is not always the general truth in every organisms. However at the present state of the science such assumption of the lineages through orthodox method is not meaningless. The exact recognition of the ontogenetic development of certain characters is generally very difficult in majority of the pelecypod species. Fortunately in Paphia the concentric lirae are useful for this purpose, for the development of the lirae of Paphia is not always in harmony with the growth of the shell itself in every species. As already cited the shells of $P$. ( $P$.) exilis. exilis are ornamented by relatively minute lirae throughout from young to adult stage; while those of $P$. (P.) exilis takaokaensis, $P$. (P.) lirata, and $P$. (P.) takanabensis are provided with comparatively coarse and strong lirae throughout the entire surface except for the umbonal area. In $P$. (P.) euglypta the lirae is very minute at the early juvenile stage but extraordinarily coarse and strong. at the adult. In other words $P$. (P.) euglypta is seemingly related to $P$. ( $P$.) exilis takaokaensis and $P$. (P.) takanabensis in the adult sculpture, but in reality it is a derivative of $P$. ( $P$.) exilis exilis as already mentioned.

Found upon the morphologic characters and the ontogenetic development of the lirae the species of Paphia (Paphia) exilis group may be separated into the three lineages, $P$. (P.) exilis exilis, exilis takaokaensis, and exilis abbreviata lineages.

The first lineage, in turn, is separated into collateral lines from $P$. $(P$.) exilis exilis Shuto to $P$. ( $P$.) papilionacea (LAMARCK), from $P$. $(P$.) naganumana (OTUKA) to $P$. ( $P$.) euglypta (PhIlIPPI), and from $P$. $(P$.) rimosa (PhILIPPI) to $(P$.) rimosa (PHILIPPI), and there is no evidence that this lineage has left. 
the direct line. The second lineage consist of the direct line from $P$. (P.) exilis takaokaensis SHuto to $P$. (P.) takanabensis Shuto and the collateral lines of $P$. (P.) lirata (PHILIPPI) and ambilis (PHILIPPI).

$P$. (P.) protolirata (Noetling) from the lowest Miocene of Burma quite resembles $P$. ( $P$.) exilis takaokaensis except for its smaller size and narrower posterior end than the latter. Considering the morphologic resemblance and the stratigraphic occurrence, the direct phylogenetic relationship between the two is assumed. Indonesian Pliocene form, $P$. (P.) rimosa (PHILIPPI) resembles the specimens of $P$. (P.) exilis exilis from the Tano area having the shorter valve with slightly coaser lirae than the types of exilis exilis.

The preliminary result of the consideration of the phylogeny of Paphia exilis group is given in table 4 .

The relation between the physico-chemical aspect of the ecology and the morphology of a few species is clarified to some extent. $P$. (P.) euglypta and $P$. (P.) naganumana, the lirae of which show the unique development as already noted, are the typical elements of the warm open water, Kuroshio fauna. In $P$. (P.) exilis exilis, ancestor of $P .(P$.$) euglypta and P .(P$.$) naganumana, the$ lirae are coarser and less numerous in the specimens from the Tano area than in those from the Aya area. The former area was affected by stronger influence of the open water than the latter in which the strong influence of the coastal water was rather predominant. $P$. $(P$.) exilis takaokaensis developed in the Tano and the other areas affected directly by the open oceanic water and its. discendants dwell altogether in the purely open warm waters.

This suggests some relations between the morphogenetic change under the

Table 4. Proposed lineages of the species group of Paphia (Paphia) exilis Shuto and $P$. (P.) hirabayashii OTUKA.

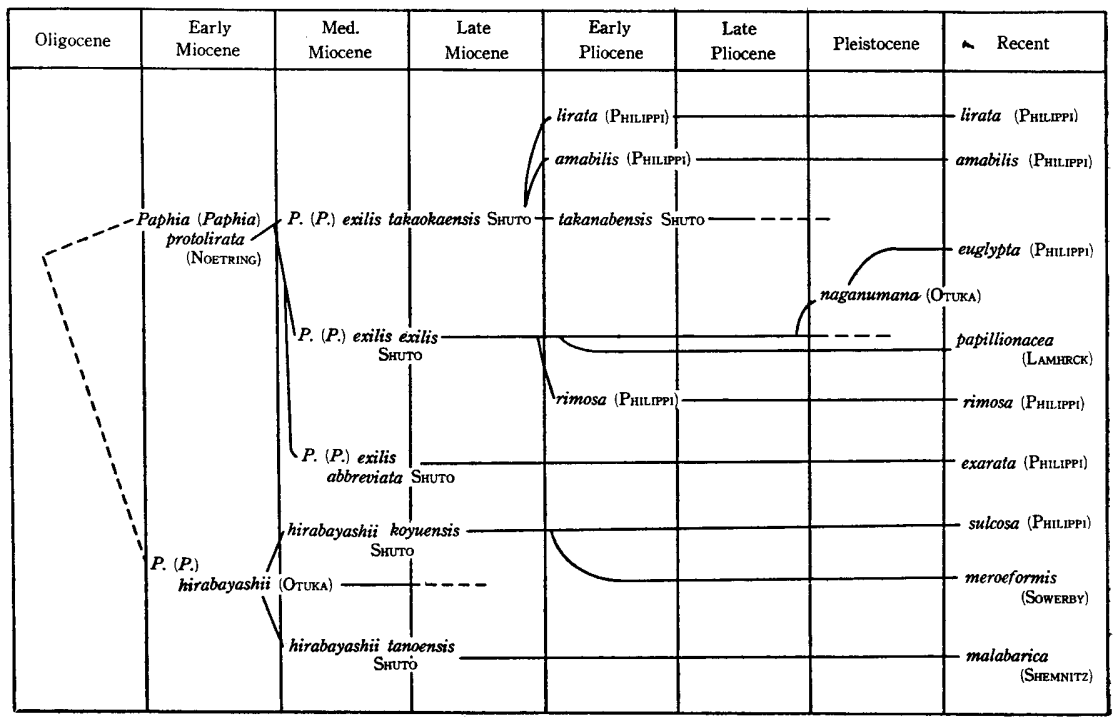


ecological control in the moderately long duration and the genetic deviation and isolation of the intraspecies demes-the real evolution in the narrow sense.

In the present schme of evolution of Paphia a few apparent tendencies are readily understood that first the discendants become larger in size than the ancestors in each lineage and the time of branching coincides with the general transgressive phases at medial Miocene, early Pliocene, and early Pleistocene.

\section{V-Adaptive evolution of Venericardia (Megacardita) panda (YoKoYAMA) group}

Venericardia (Megacardita) panda (YoKоYAMA) group is another example suggesting the adaptive evolution in the similar manner to Paphia (Paphia) exilis, though the specimens of this group are not enough in number to establish a concrete result which is comparable to the result from Paphia (Paphia) exilis SHUTO group.

V. (M.) megacostata Shuto, V. (M.) oyamai Shuto, and V. (M.) panda (Yoкочама) are closely related one another and occur respectively from the first and second, the third, and the fifth and sixth horizons of the Miyazaki group. The latter two species are distributed only in the Tsuma area, but the former not only in the Tsuma, but also in the Yusunoki and Boroishi areas. That is to say it is rather eurytopic.

The specimens of $V .(M$.$) megacostata from the Tsuma area, under the$ moderate influence of the coastal water, are featured by the unique ribs, which are eighteen to nineteen in number, quadrate in profile throughout from the early juvenile to the adult stage of growth and almost equal in breadth to the interspaces. While the specimens from the Yusunoki and the Boroishi areas, under the direct influence of the agitating open sea water, are provided with seventeen to eighteen ribs which are quadrate in the juvenile and the adolescent stages and become rounded in the adult. The intraspecies demes of $V$. (M.) megacostata are thus readily distinguished.

$V$. (M.) megacostata, oyamai, and panda are practically distinguished one another through the following diagnosis. $V$. (M.) megacostata has the smallest shells provided with the most numerous and narrowest ribs of the most quadrate profile. $V$. (M.) panda, on the contrary, has the largest shells with the least, and widest ribs of the most rounded profile. $V$. (M.) oyamai shows the typically intermediate characters between the former two.

As indicated in the text-fig. 22, the very rounded ribs of the gerontic stage of $V .(M$.$) oyamai quite agree with the adolescent-adult ribs of panda and the$ gerontic ribs of the latter are much more gently wavy. That is to say in the ontogenetic growth the rib-character at any growth stage of $V$. (M.) oyamai appears by one stage earlier in $V$. $(M$.) panda, in which finally the over-stepping feature of the same tendency is added to the adolescent development. This is an example of the hypermorphosis of de BEER.

In $V$. (M.) oyamai and $V$. (M.) megacostata from the Tsuma area the ribs are quite similar to each other until the early adolescent stage, but in the 


\begin{tabular}{|c|c|c|c|c|c|c|c|c|c|}
\hline & $V(M) M E$ & EGACOS & ATA & V.M & OYAMAI & & $V(M)$ & MPANDA & \\
\hline GEOLOGIC RANGE & Upper & or Miocer & & Mio & Pliocene & & Lower & - Pliocene & \\
\hline LENGTH & & 32 & $\mathrm{~mm}$ & & 40 & $\mathrm{~mm}$ & 50 & $\sim 60$ & $\mathrm{~mm}$ \\
\hline HEIGHT/LENGTH & 78 & $\sim 80$ & $\%$ & 75 & $\sim 79$ & $\%$ & 75 & $\sim 79$ & $\%$ \\
\hline DEPTH/LENGTH & & $\sim 33$ & $\%$ & 32.5 & $\sim 37.5$ & $\%$ & 32 & $\sim 34$ & $\%$ \\
\hline NUMBER OF RIBS & & 18 & & & 16 & & & 15 & \\
\hline UMBONAL ANGLE & $107^{\circ}$ & $\approx 115$ & & $110^{\circ}$ & $\sim 117^{\circ}$ & & & $\therefore 104$ & \\
\hline
\end{tabular}

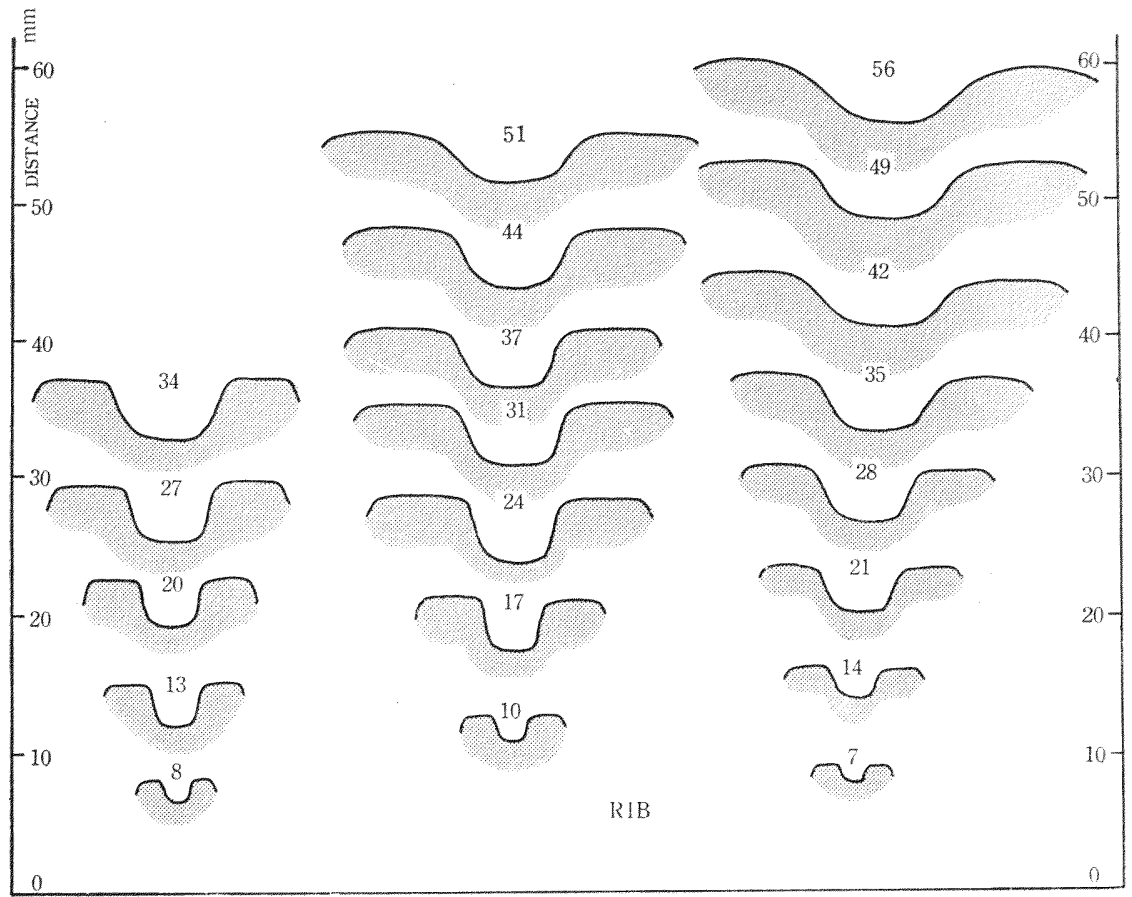

Text-fig. 22. Comparison of the species of the Venericardia (Megacardita) panda (YoKO YAMA) group.

The form characters are indicated in the upper table and the ontogenetic change of the ribs is illustrated in the lower cross sections, in which figures indicate the distance from the umbo in the term of $\mathrm{mm}$.

later stage the ribs of the former species become round in profile and wider than the interspaces, while in the latter species they develop more quickly than the interspaces. In other words the two deviate into other trend of the development at the early adolescent stage. It is very interesting that the development of the ribs of $V .(M$.$) megacostata from the Yusunoki and the Boroishi area$ bears some resemblance to the tendency of $V$. (M.) oyamai to become round in profile at the adult stage. Accordingly the open water form of $V$. (M.) megacostata is naturally considered to be a forerunner of $V$. (M.) oyamai. That is to say, $V$. (M.) megacostata includes, at least, two demes, one of which may have dwelled in the mixed water influenced by the coastal water and the other in the purely open water, and the open water species, $V$. (M.) oyamai and $V$. 
(M.) panda, are considered to have originated from the open water form of $V$. (M.) megacostata. The above mentioned facts seem to support a theory that the ecological forms appear as the result of the mutual reaction of the environmental factors and the organisms themselves, and these ecological forms, the forerunners, are fixed genetically through the effects of the everlasting environmental factors.

VI-Other examples of sympatric species among the marine animals

As indicated in the foregoing sections, polytypic species are very useful for the recognition of the process of the adaptive evolution.

E. MAYER, who urged the importance of the geographical isolation for the speciation of land animals in many papers (1947, 263-288; 1949, 281-298, etc.), also discussed it with the illustration of the marine animals, the tropic shallow water sea-urchins based on MoRTENSEN's enormous monographs (1954, 1-18). According to him "geographic speciation proceeds in two steps. During the first, a species breaks up into geographical isolates which diverge from each other genetically. The second step consists of the secondary overlap of some of these populations, without interbreeding." Hence he advocated in regards to the polytypy of the species that "the important point was that sympatric species were generally quite different, much more different than the allopatric species, so that it might have been assumed that their origin lies in the more distant past," and that "the sympatric occurrence of sibling species, such as that of Diadema setosum and of D. savignyi, was nothing exceptional." The sympatric occurrence of Diadema setosum (Rumphius) and D. savignyi (AUDouIN) is very interesting above all the examples of various allopatric and less numerous sympatric forms of echinoids which he recognized. MoRtensen distinguished six species in the genus Diadema Humphreys, of which five are allopatric and two are sympatric. The sympatric species $D$. savignyi and D. setosum are distributed from the Indian Ocean to the south Pacific altogether. Although the two are very closely similar to each other, they slightly differ in the coloration and the valves of the tridentate pedicellariae. They live in the separate colonies, but the existence of the distinct hybrids have been reported. Hence I am inclined to regard them as an example of the coexistence of the intraspecies populations like as Paphia exilis rather than the interspecies populations. Examples of the allopatric polytypy in living mollusks are numerless and those of the sympatric polytypy, even those of the closely allied forms, are not rare. Following is the selected examples of the sympatry.

Venericardia (Megacardita) kiiensis (SOWERBY) and $V$. (M.) ferruginosa (ADAMS and REEVE) are very closely allied, and are only distinguished from each other by the feature of the postero-dorsal margin which is typically rather angulated in $V$. (M.) ferruginosa and smoothly curved in $V .(M$.$) kiiensis.$ However many intermediate forms, which suggest the interconnection between the two species, were obtained from various localities. The said geographical and the geological range of $V$. (M.) kiiensis is included in those of $V$. (M.) 
ferruginosa, and the population density of the former is always much thinner than that of the latter in the overlapping area. Hence there is no concrete base to rank the two as good and different species, although they belong, of course, to different demes respectively.

Three Japanese species of Crassatellites (Crassatellites), C. (C.) adamsi (Kobelt), C. (C.) heteroglyptus PILsBRy, and C. (C.) nanus (AdAMs and REEVE) also so closely resemble one another that some malachologists treat them as synonymous. Distinction of the typical forms of three species is practically carried out. C. (C.) nanus attains the largest size with the dimensions below noted, and its shell is featured by the sculpture consisting of regular concentric ribs throughout the entire surface and by the somewhat concave ventral margin just before the rostration in fully grown shells $C$. (C.) heteroglyptus is medium in size and its weak concentric ribs become obsolete and irregular after adolescent stage is reached. C. (C.) adamsi is smallest in size and the ribs are of intermediate strength and regularity between the foregoing two. The dimensions are as follows in the adult specimens of the standard form:
C. (C.) nanus
$\mathrm{L}=40-45 \mathrm{~mm} ., \mathrm{H} / \mathrm{L}=86-88 \%, \mathrm{D} / \mathrm{L}=43-45 \%$
C. (C.) heteroglyptus
$\mathrm{L}=30-35 \mathrm{~mm} ., \mathrm{H} / \mathrm{L}=80 \pm \%, \mathrm{D} / \mathrm{L}=40 \pm \%$
C. (C.) adamsi
$\mathrm{L}=20 \mathrm{~mm}$,
$\mathrm{H} / \mathrm{L}=76-79 \%, \mathrm{D} / \mathrm{L}=42 \pm \%$

Among three end forms there are various and complicate intermediate forms as well known. C. (C.) adamsi is distributed in Japan Sea from Tsushima to Fukui and in the Pacific off Bôsô. C. (C.) heteroglyptus is distributed in the Pacific from Kyushu to Bôsô and in Japan Sea from Tsushima to Sado. C. (C.) nanus, which has the widest geographical range, is longest in the geological range, being back to the lower Pliocene.* I am interested in the analogy of distribution, both geographical and geological, between Japanese Crassatellites and Paphia exilis group.

There are other examples which represent different stages of the adaptive speciation. Turcicula argenteonitens (LISCHKE) is distributed on the upper continental slope of the Pacific side of Japan from Kyushu to Sanriku (N. 33 to 41). This species contains two distinct groups of populations; one is distributed north of Bôsô in the Oyashio waters and is called T. argenteonitens hirasei KURODA, and the other, T. a. argenteonitens (LISCHKE), is distributed south of Bôsô in the Kuroshio waters. The morphologic criterion to separate two forms is that the spirally arranged nodules at the middle of the whorls are rather sharp and developed on the whole whorls except for the earliest ones in $T$. argenteonites hirasei, but they are rather blunt and developed only on the penultimate and the last whorl of the adult shells in T. argenteonitens argenteonitens. The typical forms are thus practically and easily distinguished each other. However, on one hand, the population of T. argenteonitens argenteonitens contains the specimen which are quite similar to T. argenteonitens hirasei and also the specimens utterly devoid of the nodules. On the other hand the population of $T$. argenteonitens hirasei contains some specimens similar to $T$. argenteonitens

\footnotetext{
* Pliocene of Formosa (Byoritsu heds) and Okinawa (Shimajiri group).
} 


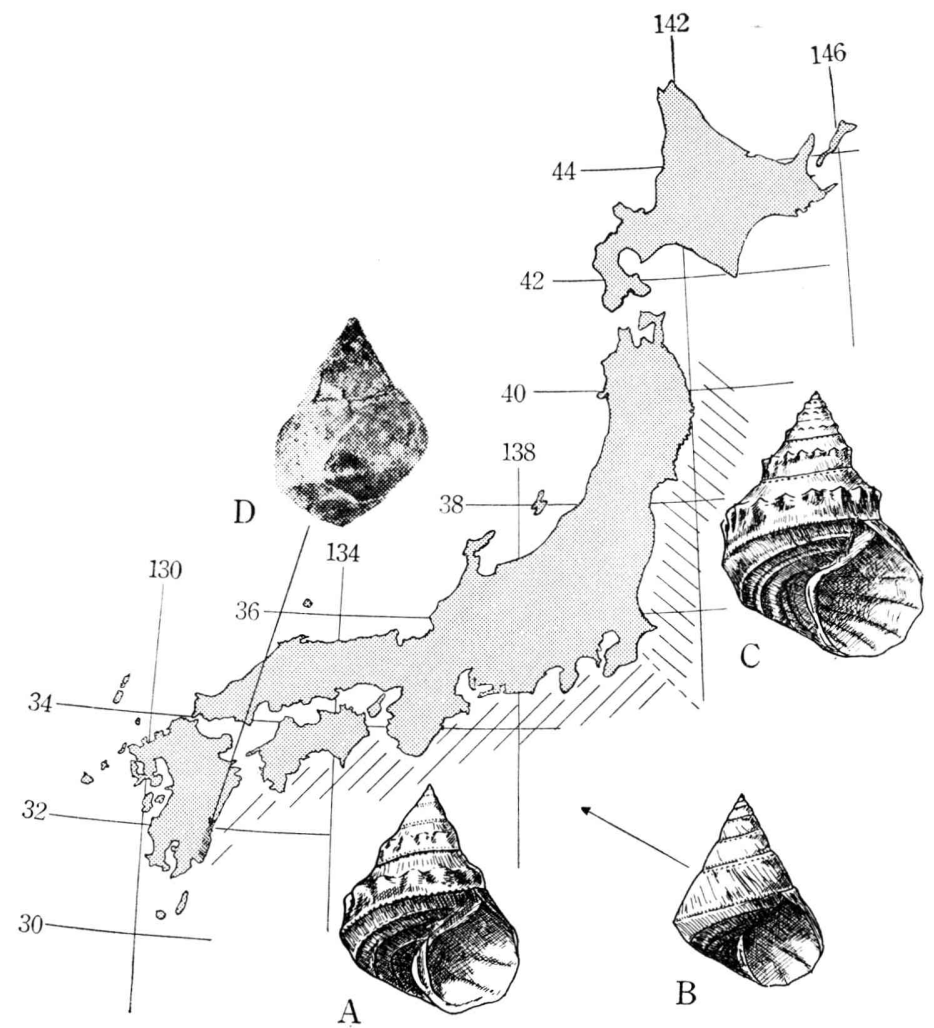

Text-fig. 23. Three forms of Turcicula argenteonitens (LISCHKE) and their geographical distribution.

A...T. argenteonitens argenteonitens (LISCHKE),

B....small and nodule-free form obtained from the population of $T$. argenteotitens argenteonitens.

C....T. argenteonitens hirasei KurodA,

D....T. argenteonitens convexiuscula (YoKoyAMA) from the uppermost horizon (sixth horizon) of the Miyazaki group. It quite agrees with B.

argenteonitens. Whereas the Pliocene form, T. argeneonitens convexiuscula (YoKоYAMA) quite resembles T. argenteonitens argenteonitens except for the smaller size and the nodule-free whorls, and is considered by the Japanese malachologsits as the ancestor of the latter. The former is distributed in the Pliocene beds of the Pacific side of our country from Kyushu to Bôsô with the association of the molluscan assemblages of the lower shelf. The morphologic features and the habitats of the three subspecies may be briefly written as follows.

T. a. convexiuscula [ancestor], subtropical waters, lower shelf; smaller size, nodule-free.

T. a. argenteonitens [off spring], present Kuroshio waters, upper part of the continental slope; larger size, nodules developed only on the late 
whorls.

T. a. hirasei [off spring], present Oyashio waters, upper part of the continental slope; largest, nodules fully developed.

This group may illustrate that the warm water form invaded adaptically into the cool waters to establish a new form. Ancestral form in the warm water left two distinctive subspecies; the one is a dweller in the less warmer and deeper water than the ancester lived and the other is typically cold water

Table 5. Hypothetic scheme of the speciation process of the pelecypods. Enthusiasm is placed on the correspondence between the phenomena in pelecypods and those in the environment.

SPECIATION PROCESS OF PELECYPODA

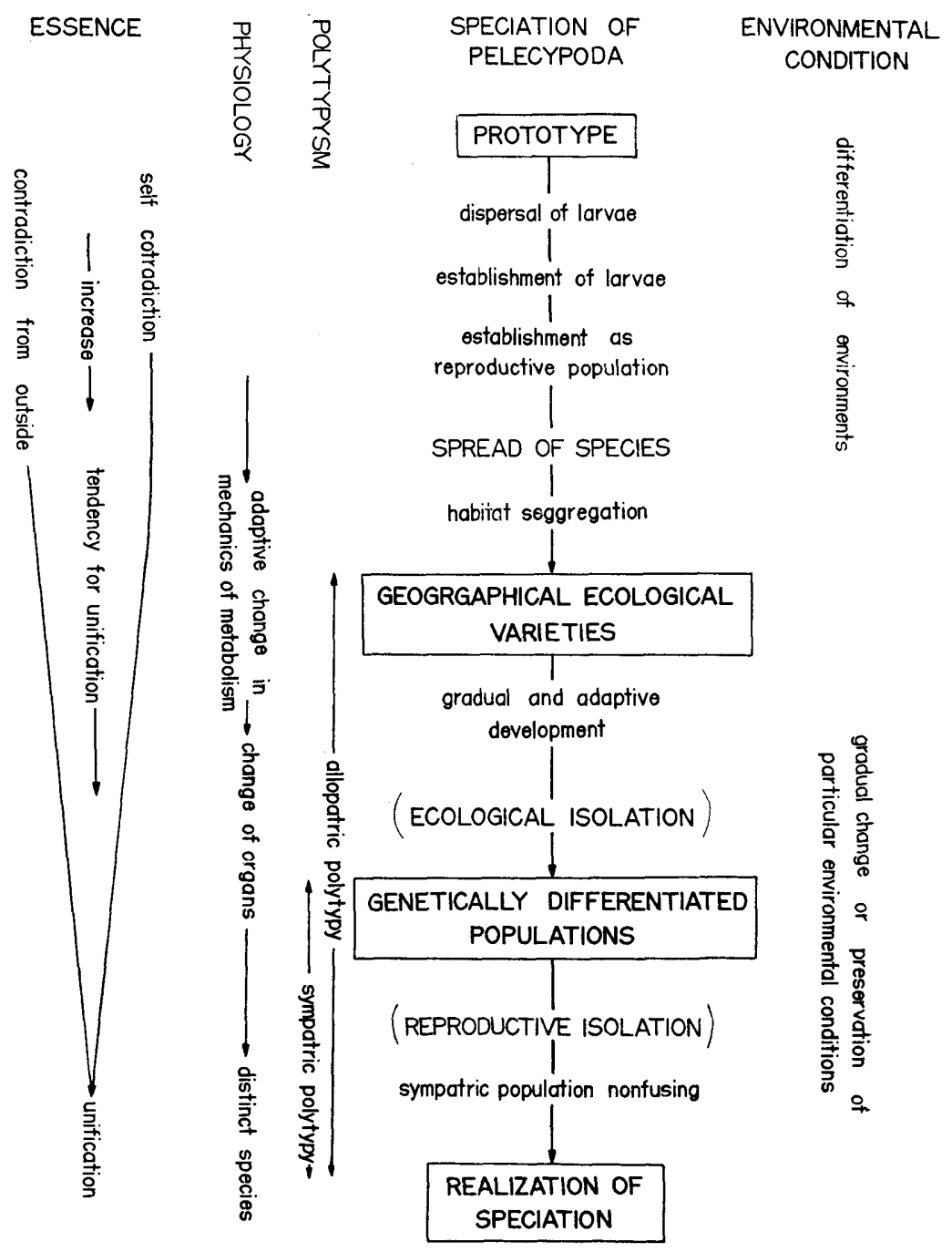


subspecies. Namely the facts suggest the make up of the geographical isolates which, according to MAYER, is the first step of the geographical speciation.

These examples may respectively represent the different steps of adaptive speciation including the mere ecological and temporary isolation illustrated by Joannisiella cumingii (HANLEY) etc., the imperfect genetical isolation illustrated by Turcicula argenteonitens (LISCHKE) group, and the moderate genetical isolation illustrated by Paphia (Paphia) exilis SHUTo group.

\section{VII-Concluding remarks}

For the conclusion of the observations above mentioned, a hypothetical scheme of the speciation process of the marine pelecypods is offered in table 5.

This scheme of the speciation is not entirely new and original, but it contains partially almost the same frame works as those of certain authors (MAYER, 1947, 263-288, Sylvester-BradLeY, 1951, 88-102) as well as the ideas newly introduced here.

Intraspecies allopatry of certain eurytopic species corresponding with the environmental divergence is, as already pointed out, available for the consideration of the speciation of the organisms, and moreover the examination of such polytypic species as Paphia (Paphia) exilis SHUTo, exhibiting partially allopatric and partially sympatric at the same time, enables us to approach the analysis of the process and the phase of the speciation of a species, which the orthodox method could not inquire into.

From the phenomenological stand of view the speciation may be explained as following. The prototype spreads at first through the dispersal of the velliger larvae. If the new environment where the larvae are brought about is favourable for their lives, they settle and establish themselves there to result the expansion of the occupation of this species. In nature every species consists generally of various populations, which are differentiated ecologically but not genetically and occupy favourable habitats respectively. Because of the homogenety of the heredity, irrespective of the morpho-ecological divergency, the velliger larvae or settled larvae of the particular population may develop to other morpho-ecological form which adapts to new environment and characterizes it, if they are artificially brought into different but not unsuitable environment. Several experiments of this kind have been offered before us.

If any environment is maintained or progressively developed along the same tendency, the adaptability of the pelecypod species, which dwell in that environment, may be accelerated through the change in the mechanics of the metabolism. In other words among the populations which respectively adapt to different environments the merely ecological isolation develops gradually into the genetical isolation through the adaptive change in the metabolism.

The substance of these phenomena is understood as following. In the case that the external factors, acted upon organism from outside, change to some extent, the harmony between the organisms and the environment is broken down, resulting in the inevitable contradiction to the lives of the organisms. 
Corresponding to the external contradiction some tension breaks out within the organism itself. This tension is called interior contradiction. While the organisms has the tendency not merely to sustain the tension, but to release itthe tendency to harmonize with the external stimulus. This is realized through the change in the mechanisms of metabolism of the organisms. When the contradictions, both internal and external, are synthetized-when the physical constriction of the organisms changes to adapt perfectly to the environmentthe speciation is completed.

I treated here chiefly the sedimentary rocks and the pelecypod fossils for the basis of the consideration of the speciation. Accordingly the result of the consideration can not be necessarily applied to the speciation phenomena of other organisms which differ from the marine pelecypods in physiology and ecology. However the concept and the frame work included in this hypothesis are significant to be examined by other examples. 


\section{Works Cited}

Abel, Othenio (1929): Palaeobiologie und Stammesgeschichte. Gustav Fischer, Jena. 1-423, 224 text-figs.

Akers, W. H. (1955): Some Planktonic Foraminifera of the American Gulf and Suggested Correlations with the Caribbean Tertiary. Jour. Paleont. 29, (4), 647-664, 1 pl., 3 text-figs.

A kers, W. H. and Drooger, C. W. (1957): Miogypsinids, Planktonic Foraminifers, and Gulf Coast Oligocene-Miocene Correlation. Amer. Assoc. Petrol. Geol. 41, 656-678, 2 text-figs.

Beadle, L. C. (1931): The Effect of Salinity Changes on the Water Content and the Respiration of Marine Invertebrates. Jour. Exper. Biol. 8, (3), 211-227, 6 text figs.

Bellardi, L and Sacco, F. (1872-1904): Molluschi dei Terreni Terziari del Piemonte e della Liguria. 30 fas.

Bemmelen, R. W. van (1949): The Geology of Indonesia Vol.1, A General Geology of Indonesia and Adjacent Archipelagoes. Government Printing Office, Hague. 1-732, 377 text-figs.

Beurlen, Karl (1937): Die Stammesgeschichtlichen Grundlagen der Abstammungslehre. Gustav Fischer, Jena. 1-264.

Blow, H. W. (1959): Age, Correlation, and Biostratigraphy of the Upper Tocuyo (San Lorenzo) and Pozón Formations, Eastern Falcón, Venezuela. Bull. Amer. Paleont., 39, (178), 1-251, 19 pls.

Bolli, Hans M., Loeblich, A. R. Jr., and TapPen, H. (1957): Planktonic Foraminiferal Families Hantkeninidae, Orbulinidae, Globorotaliidae, and Globotruncanidae. U. S. Nat. Mus. Bull., (215), 3-50, 9 text-figs., $11 \mathrm{pls.}$

CaIN, J. A. (1952): Geography, Ecology and Coexistence in Relation to Biological Definition of Species. Evolution, 7, (1), 76-83.

Cooke, C. W., Gardner, Julia, and Woodring, Wendel P. (1943): Correlation of the Cenozoic Formations of the Atlantic and Gulf Coastal Plain and the Caribbean Region. Geol. Soc. Amer. Bull., 54, 1713-1722, 1 pl.

Cossmann, Maurice and Peyrot, A. (1900-33): Conchologie Néogènique de l'Aquitaine. Actes Soc. Linn. Bordeaux. 6 vols., 124 pls.

Cushman, Joseph Augustine (1914): A Monograph of the Foraminifers of the North Pacific Ocean. Pt. 4, Chilostomellidae, Globigerinidae, Nummulitidae. U. S. Nat. Mus. Bull., (71), 1-43, 19 pls.

Davies, A. Morley (1934): Tertiary Faunas, Vol. 2, The Sequence of Tertiary Faunas. Thomas Murby and Co., London. 1-252, 28 text-figs.

DeHM, R. (1949) : Zur Oligocän-Miocän Grenze. N. Jahrb. f. Min., Paläont., etc., Monatsh. Abt. B, Jahrg. 1949, 141-146.

DePeret, C. (1902-1912): Monographie des Pectinidés Néogènes de L'Europe et des Régions Voisines. Soc. Geol. France Mem., (26), 1-168, 71 text-figs., 2 tables, 23 pls.

Dobzhansky, Theodosius (1951): Genetics and Origin of Species (3rd Ed.), Columbia Univ. Press, New York, 1-364, 23 text-figs., 15 tables.

Dollfus, G. F. and Dautzenberg, P. (1902-20): Conchyliologie du Miocene Moyen du Bassin de la Loire. Soc. Geol. France Mem., (27), Pelecypodes, 1-500, 51 pls.

Drooger, C. W. (1954): The Oligocene-Miocene Boundary on Both Sides of the Atlantic. Geol. Mag., 91, 514-518, 1 text-fig.

Durham, J. Wyatt (1958): Palaeoclimates (in Physics and Chemistry of the Earth, L. H. Ahrens et al. ed.), Pergamon Press, London, 1-16, 8 text-figs. 
EAmes, F. E. (1953): The Miocene/Oligocene Boundary and the Use of the Term Aquitanian. Geol. Mag., 90, (6), 388-399.

EkMAN, S. (1953): Zoogeography of the Sea. (Translated in English by Elizabeth PALMER). Sedgwick and Jackson. 1-417, 121 text-figs., 49 tables.

Elton, C. (1953) : Animal Ecology, Sedgwick and Jackson. 1-207, 13 text-figs., 8 pls. Fischer, P. J. (1921) : Eine Pliocänfauna von Seran (Molukken). Zentral blatt $f$. Min. Geol. u. Paläont. Jahrg. 1921, 242-251.

(1927) : Beiträg zur Kenntnis der Pliozänfauna der Molukkeninseln Serran und Obi. Paläontologie von Timor, Lief. 15, 1-179, 6 pls.

Fleming, C. A. (1957): The Genus Pecten in New Zealand. New Zealand Geol. Surv. Paleont. Bull., (26), 1-69, 13 text-figs., 15 pls.

Florkin, Marcel (1949) : Biochemical Evolution (ed. Sergius Morgulius), Academic Press, 1-157, 25 text-figs., 13 tables.

Fujita, Tadashi (1929): Report on the Dredged Shells of Tateyama Bay. Pt. 1 and 2. Venus 1, (2), 58-65 and 1, (3), 88-92, 3 text-figs., 1 pl.

CARdner, Julia (1924): Coastal Plain and European Miocene and Pliocene Mollusks. Geol. Soc. Amer. Bull., 35, 857-866.

, (1926-28): The Molluscan Fauna of the Alum Bluff Group of Florida. U. S. Geol. Surv. Prof. Paper (142), 1-249, 36 pls.

Gekker, R. Th. (1957): Vvedenie v Paleoekologiyu (transl. in Japanese by Transl. Subcommittee in the Assoc. Geol. Collab. Japan). 1-204, 30 text-figs., 20 pls.

Gignoux, Maurice (1936): Géologie Stratigraphique (2me ed.). Masson, Paris, $1-709,145$ text-figs.

Glaessner, Martin F. (1951): Three Foraminiferal Zones in the Tertiary of Australia. Geol. Mag., 88, 273-283.

(1953): Time Stratigraphy and the Miocene Epoch. Geol. Soc. Amer. Bull., 64, (6), 647-658, 1 table.

Gregory, J. W., Cox, L. R., and Currie, Ethel D. (1924): The Geology of Aru Islands. Geol. Mag., 61, 52-72, 4 text-figs., 1 pl.

Gunter, Gordon (1957): Temperature. (in Treatise in Ecology and Paleoecology. Vol. 1, Ecology). Geol. Soc. Amer. Mem., (67), 159-183, 3 text-figs.

HAMAI, Ikuzo (1934): On the Local Variation on the Shells of Meretrix meretrix (L.), with Special Reference to Growth of Organism. Sci. Rep. Tohoku Imp. Univ., Ser. 4, 9, (2), 131-158, 6 text-figs.

- _ (1935): A Study of One Case in Which Different Environmental Conditions Produce Different Types of Merctrix meretrix (L.). ibid. 10, (3), 485-498, 8 text-figs.

Hanzawa, Shoshiro (1935): Some Fossil Operculina and Miogypsina from Japan and Their Straitigraphic Signification. ibid. Ser. 2, 18, (1), 1-29, 3 pls.

HARMer, F. W. (1898) : Pliocene Deposits of the East of England Pt. 1, The Lenham Beds and the Coralline Crag. Quart. Jour. Geol. Soc. London, 56, 705-738, 6 text-figs.

HeddberG, H. D. (1948) : Time-Stratigraphic Classification of Sedimentary Rocks. Geol. Soc. Amer. Bull. 59, (5), 447-462.

-, (1951): Nature of Time-Stratigraphic Units and Geologic Time Units. Amer. Assoc. Petrol. Geol. Bull., 35, 1077-1081.

HEDGPETH, Joel W. (1957) : Marine Biogeography. (in Treatise in Ecology and Paleoecology, Vol. 1, Ecology). Geol. Soc. Amer. Mem. (67), 359-382, 16 text-figs., $1 \mathrm{pl}$.

Horikoshi, Masuoki (1957): Note on the Molluscan Fauna of Sagami Bay and its Adjacent Waters. Sci. Rep. Yokohama Nat. Univ. Sec. 2, (6), 37-64, 13 text-figs., 1 pl., 2 charts. 
IDA, Kazuyoshi (1952): A Study of Fossil Turritella in Japan. Geol. Surv. Japan Rep. (150), 1-64, 25 text-figs., 7 pls.

, (1956): A New Graphic Method Indicating the Character of Fossil Molluscan Fauna Geol. Surv. Japan Bull., 7, (2), 15-20, 10 text-figs., 1 table.

IGI, Josei (1904): Geological Map Sheet and Explanatory Text, Sadowara (1/200000). 1-42, 3 text-figs., 2 pls.

IJIRI, Shoji (1952): Fundamental Problems in Geology (in Japanese). Earth Science Series from Assoc. Geol. Collab. Japan, (5), 1-58, 7 text-figs., 4 tables.

, 1954): On Science (in Japanese). Rironsha, Tokyo. 1-308, 13 text-figs.

IKeBe, Nobuo (1954): Cenozoic Biochronology of Japan. Jour. Inst. Polytechnics, Osaka City Univ. 1, (1), 73-86, 5 text-figs., 2 tables.

Itorgawa, Junji (1953): A New Species of Fossil Siphonalia from the Miyazaki Group. Venus (Japan. Jour. Malachology) 17, (4), 213-218, 4 text-figs.

Jeletzky, J. A. (1956): Paleontology, Basis of Practical Geochronology. Amer. Assoc. Petrol. Geol. Bull. 40, (4), 679-706.

Joysey, K. A. (1952): Fossil Lineages and Environmental Change. Geol. Mag. 89, (5), $357-360$.

Katto, Jiro and Ozaki, Hiroshi (1955): Miocene Nobori Formation of Kochi Prefecture, Japan. Res. Rep. Kochi Univ. 4, (1) , 1-7, 4 text-figs., 3 tables.

Keen, A. Myra (1940): The Percentage Method of Stratigraphic Dating. Proc. 6th Pacific Sci. Congr. Pacific Sci. Assoc. 2, 659-663, 2 text-figs.

KeLleR, Gerhard (1956): Paläotopographie und Kleinfacies. Geol. Rundsch. 45, (1), 128-134.

Kinoshita, Toraichiro (1932): Dredged Shells from the Off Shore Waters of Kii. Venus (Japan. Jour. Malac.) 3, (5), 251-259, 1 text-fig.

Kitazaki, Umeka, Yagishita, Hideharu, and Araki, Harumi (1955): Convenient Method of the Measurement of the Organic Content in the Sedimentary Rocks by Means of D.T.A. Res. Inst. Nat. Resources Misc. Rep. (37), 11-18, 9 text-fig.

KITOU, Masataka (1956): Vertical Distributions of Planktonic Larvae of Littoral Animals and Neritic Zooplanktons. Oceanographical Mag. 3, (2), 93-95, 4 text-figs.

Korzumi, Masami (1957): On the Forecast of Oceanographic Conditions in the Seas West of Japan. ibid. 9, (1), 43-49, 2 text-figs.

KoтAка, Tamio (1958): Faunal Consideration of the Neogene Invertebrates of Northern Honshu, Japan. Saito Ho-on Kai Mus. Res. Bull. (27), 38-44, 1 textfig., 3 tables.

- , (1959): The Cenozoic Turritellidae of Japan. Sci. Rep. Tohoku Univ. Ser. 2, 31, (2), 1-135, 11 text-figs, $15 \mathrm{pls}$

Kuroda, Tokubei (1941): A Catalogue of Molluscan Shells from Taiwan (Formosa), with Descriptions of New Species. Mem. Fac. Sci. \& Agri. Taihoku Imp. Univ. 22, (4), 65-216, 7 pls.

Kuroda, Tokubei and HABE, Tadashige (1952): Check List and Bibliography of the Recent Marine Mollusca of Japan. Tokyo, 1-210.

Le Roy, L. W. (1948): The Foraminifer Orbulina universa d'Orbigny, a Suggested Middle Tertiary Time Indicator. Jour. Paleont. 22, 500-508, 4 text-figs.

Maelennan, Rhone M. (1945): Generations in a Fossil Community. Geol. Mag. 82, (4), 177-181.

MakiYama, Jiro (1924): The Evolution of Umbonium. Japan. Jour. Geol. Georg. 3, (3-4), 119-130, 3 text-figs., $1 \mathrm{pl}$.

(1927): Molluscan Fauna of the Lower Part of the Kakegawa Series in the Province of Totomi, Japan. Mem. Coll. Sci. Kyoto Imp. Univ. Ser. B, 3, (1) (Art. 1), 1-147, 6 pls. 
, (1931): Stratigraphy of the Kakegawa Pliocene in Totomi. ibid. 7, (1), (Art. 1), 1-53, 4 text-figs., 3 pls.

(1935) : Morphogenetic History of Umboniidae (in Japanese). Venus (Japan. Jour. Malac.) 5, 241-255, 2 text-figs.

(1940): The Neogenic Stratigraphy of Japan Islands. Proc. 6th Pacific Sci. Congr. Pacific Sci. Assoc. 2, 641-649, 3 text-figs., 1 pl.

MANSFIEld, W. C. (1936) : Stratigraphic Significance of Miocene, Pliocene and Pleistocene Pectinidae in the Southeastern U. S. Jour. Paleont. 10, 665-668.

MARTIN, K. (1879-80): Tertiaerschichten auf Java. Leiden, 1-164, 16 pls.

, (1881): Jungtertiaere Ablagerungen in Padangschen Hochlande auf Sumatra. Samml. Geol. Reichs-Mus. Leiden, Ite Ser. 1, 84-104, 2 pls.

, (1913): Einige Algemeinere Betrachtungen über des Tertiäre von Java. Geol. Rundsch. 4, (3), 161-173.

(1914): Miocene Gastropoden von Ost-Borneo. Samml. Geol. Reichs-Mus. Leiden, Ite Ser. 9, 326-336, 1 text-fig.

Marwick, J. (1957) : New Zealand Genera of Turritellidae, and the Species of Stiracolpus. New Zealand Geol. Surv. Paleont. Bull. (27), 1-55, 5 text-figs., 2 tables, 5 pls.

MAYER, Ernst (1942): Systematics and the Origin of Species. Columbia Univ. Press, New York. 1-334, text-figs.

- , (1947): Ecological Factors in Speciation. Evolution 1, (4), 263-288. 1 text-fig.

, (1949): Speciation and Systematics (in Genetics, Paleontology, and Evolution ed. G. L. JePson, G. G. Simpson, and E. MAYER) 281-298, 3 text-figs.

, (1954): Geographic Speciation in Tropical Echinoids. Evolution, 8, (1), 1-8, 7 text-figs.

(1955): Systematics and Modes of Speciation. Biological Systematics. Proc. 16th Annual Biology Colloqium. 45-51.

, (1957): Species Concepts and Definitions (in The Species Problem). 1-22.

Metcalf, M. M. (1930): Salinity and Size. Science 72, 526-527.

Miller, Robert L. (1955): The Statistical Stability of Quantitative Properties as a Fundamental Criterion for the Study of Environments. Jour. Geol. 63, (4), 376-387.

Mizuno, Atsuyuki (1957): Cenozoic Sedimentary Provinces in Japan and their Evolution. (3) Tertiary History of the Pacific side of Southwest Japan. Bull. Assoc. Geol. Collab. Japan (Shinseidaino Kenkyu) (24-25), 28-41, 7 text-figs., 6 tables.

MooRe, Hilary B. (1958): Marine Ecology. Wiley, New York. 1-493, 243 text-figs., 72 tables.

Moore, Raymond C. (1949): Meaning of Facies. Geol. Soc. Amer. Mem. (39), 1-34, 21 text-figs.

(1957): Modern Method of Paleoecology. Amer. Assoc. Petrol. Geol. Bull. 41, (8), 1775-1801.

Moriyasu, Shigeo (1956): On the Oceanographical Conditions South of Honshu in 1954. Oceanographical Mag. 7, (2), 105-113, 6 text-figs.

Mortensen, Th. (1940): A Monograph of the Echinoidea III-1, Aulodonta. C. A. Reitzel Publ. Copenhagen. 1-370, 197 text-figs., 77 pls.

Newcombe, C. L. and Kessler, H. (1936): Variations in Growth Indices of Mya arenaria L. on the Atlantic Coast of North America. Ecology, 17, (3) , 429-443.

Noetling, Fritz (1901): The Fauna of the Miocene Beds of Burma. Palaeontologia Indica N.S. 1, (3), 1-378, 25 pls.

Nomura, Sitihei (1933): Catalogue of the Tertiary and Quartery Mollusca from the 
Islad of Taiwan (Formosa) in the Institute of Geology and Paleontology, Tohoku Imperial University, Sendai, Japan. Sci. Rep. Tohoku Imp. Univ. Ser. 2, 16, 1-106, 4 pls.

(1936): Catalogue of the Tertiary and Quartery Mollusca from the Island of Taiwan (Formosa) in the Institute of Geology and Paleontology, Tohoku Imperial University, Sendai, Japan Pt. 2, Scaphopoda and Gastropoda. ibid. $18,53-228,5 \mathrm{pls}$.

(1937): The Molluscan Fauna from the Pliocene of Tosa. Japan. Jour. Geol. Geogr. 14, (3-4), 67-90, $1 \mathrm{pl}$.

Nomura, Sitihei and Zimbo, Noboru (1935): Molluscan Fossils from the Shimajiri Beds of Okinawa-zima, Ryukyu Islands. Sci. Rep. Tohoku Imp. Univ. Ser. 2, $18,226-266,1 \mathrm{pl}$.

Odum, Eugene P. (1953): Fundamentals of Ecology. W. B. Saunders, New York. 1-432, 119 text-figs., 19 tables.

Ogose, Sunao (1959): On Some Problems of Percentage Method. (in Japanese). Jour. Geol. Soc. Japan 65, (761), 90-105, 11 tables.

Oostingh, C. H. (1935): Die Mollusken des Pliozän von Boemiajoe (Java). Wet. Meded. 26, 1-247, 17 pls.

Otsuka, Senichi (1900): Geological Map Sheet and Explanatory Text (1/200000), Miyazaki. (in Japanese). 1-31, 1 text-fig.

OтUkA, Yanosuke (1930): Some Geological Problems at the Takanabe District, Miyazaki Prefecture. (in Japanese). Geogr. Rev. 6, (7), 496-522, 8 text-figs.

-_, (1934): Tertiary Structures of the Northwestern End of the Kitakami Mountainland, Iwate Prefecture, Japan. Bull. Earthq. Res. Inst. 12, (3), $566-638,8$ pls.

- - (1947): On the Sakuma Group of Boso Peninsula (in Japanese). Jour. Geol. Soc. Japan 53, (622-627), 94.

, (1949): Fossil Mollusca and Rocks of the Kiyosumi Group Exposed at Minato-machi, Chiba Prefecture and its Environs. Japan. Jour. Geol. Geogr. 21, (1-4) , 295-314, 3 text-figs., 1 pl.

Oyama, Katura (1952): On the Vertical Distribution of Marine Mollusca. (in Japanese). Venus (Japan. Jour. Malac.), 17, (1), 27-35.

- _ (1953-a): Studies on Fossil Molluscan Biocoenosis: on the Fossil Communities of the Coastal Water (No. 1). (in Japanese). Misc. Rep. Res. Inst. Nat. Resources, (31), 54-59, 2 tables.

, (1953-b): Studies on Fossil Molluscan Biocoenosis: Fossil Communities of the Oceanic Waters (No. 1). (in Japanese). ibid. (32), 23-30, 1 table.

, (1954-a): Studies on Fossil Molluscan Biocoenosis: On the Fossil Communities of the Coastal Water (No. 2). (in Japanese). ibid. (33), 92-99, 1 table.

, (1954-b) : Stratigraphical View for Pecten Ecology. $19^{\circ}$ Congr. Geol. Intern. Comptes Rendus, Sect. 13, Fasc. (15), 97-99.

, (1954-c): Studies on Fossil Molluscan Biocoenosis: On the Fossil Communities of the Oceanic Water (No. 2). (in Japanese). Misc. Rep. Res. Inst. Nat. Resources, (34), 89-100, 2 tables.

- (1955): A Contribution to the Phylogeny of Umboniinae (in Japanese). Venus (Japan. Jour. Malac.) 18, (3), 169-176.

PaIro, M. Crusafont (1951): El Sistema Miocénico en la Depressión Espanõla del Vallés-Penedés. Intern. Geol. Congr. Rep. Sec. 18, Pt. (11), 34-42.

PARKER, Robert H. (1956): Macro-Invertebrate Assemblages as Indicators of Sedimentary Environments in East Mississippi Delta Region. Amer. Assoc. Petrol. Geol. Bull. 40, (2), 295-376, 32 text-figs., 8 pls.

-_, (1959): Macro-Invertebrate Assemblages of Central Texas Coastal Bays. 
and Laguna Madre. ibid. 43, (9), 2100-2166, 32 text-figs., 6 pls.

Pearse, A. S. and Gunter, Gordon (1957): Salinity (in Treatise on Marine Ecology and Paleoecology Vol. 1, Ecology). Geol. Soc. Amer. Mem. (67), 129-158, 7 text-figs.

Pensch, Bernhard (1948): Histological Changes Correlated with Evolutionary Changes of Body Size. Evolution 2, (2), 218-230, 14 text-figs.

Philippi, H. E. (1898): Beiträge zur Morphologie und Phylogenie der Lamellibrachier. Zeitschr. Deut. Geol. Geselsch. 50, 597-622, 7 text-figs.

-_, (1900): Beiträge zur Morphologie und Phylogenie der Lamellibranchier. ibid. 52, 64-117, 24 text-figs.

Polski, William (1959): Foraminiferal Biofacies off the North Asiatic Coast. Jour. Paleont. 33, (4), 569-587, 8 text-figs., 3 tables.

Powell, A. W. B. (1940): Note on the Importance of Recent Animal Ecology as a Basis of Paleoecology. Proc. 6th Pacific Sci. Congr. Pacific Sci. Assoc. 2, $607-617,3$ pls.

Revelle, Roger and Fairbridge, Rhodes (1957): Carbonates and Carbon Dioxide (in Treatise on Marine Ecology and Paleocology Vol. 1, Ecology), Geol. Soc. Amer. Mem. (67), 239-296, 8 text-figs.

Roger, Jean (1939): Le Genre Chlamys dans les Formations Néogènes de L'Europe. Mém. Soc. Géol. France N. Ser. (40), 1-294, 113 text-fig.s., 28 pls.

Roemer, Eduard (1800): Monographie der Mollusken Gattung Venus 2, Tapes, 38 pls.

Schenck, Hubert G. (1936): Nuculid Bivalves of the Genus Acila. Geol. Soc. Amer. Spec. Pap. (4), 1-149, 13 text-figs., 17 tables, 17 pls.

Schindewolf, Otto H. (1950-a): Grundlagen und Methoden der Paläontologische Chronologie, 3e Auflage. Schweizerbart'sche, Stuttgart. 1-152, 48 text-figs.

, (1950-b): Grundfragen der Paliiontologie. Schweizerbart'sche, Stuttgart. 1-506, 332 t€xt-figs., 32 pls.

(1950-c): Zeitfaktor in Geologie und Paläontologie. Schweizerbart'sche, Stuttgart. 1-114, 18 text-figs.

Shuto, Tsugio (1952): Stratigraphical Study of the Miyazaki Group (in Japanese). Sci. Rep. Fac. Sci. Kyushu Univ. Geology. 4, (1), 1-40, 4 text figs., 4 tables, $8 \mathrm{pls}$.

, (1955): Amussiopecten from the Miyazaki Group, Miyazaki Prefecture (Palaeontological Study of the Miyazaki Group-2). Trans. Proc. Palaeont. Soc. Japan N.S. (20), 101-110, 4 text-figs., 2 pls.

(1956): Umboniinae from the Miyazaki Group (Palaeontological Study of the Miyazaki Group-1). Japan. Jour. Geol. Geogr. 27, (1), 47-66, 7 text-figs., 4 tables, 1 pl.

(1957-a): Crassatellites and Venericardia from the Miyazaki Group (Palaeontological Study of the Miyazaki Group-4). Mem. Fac. Sci. Kyushu Univ. Ser. D, Geol. 6, (2), 69-89, 7 text-figs., 3 tables, 1 pl.

, (1957-b) : Polymorphism in Mollusks and Facies Differentiation (Palaeontological Basis for the Consideration of Speciation-I). (in Japanese with English Abstract). Jour. Geol. Soc. Japan 63, (745), 565-685, 17 text-figs., 3 tables. (1957-c) : Palaeontological Aspects of Speciation (Palaeontological Basis for the Consideration of Speciation-II). (in Japanese with English Abstract). ibid. 63, (746), 636-647, 2 text-figs., 2 tables.

, (1957-d): Fossil Paphia from the Miyazaki Group (Palaeontological Study of the Miyazaki Group-3). Japan. Jour. Geol. Geogr. 28, (1-3), 139-160, 9 text-figs., 6 tables, 1 pl.

(1958-a): Granulifusus from the Miyazaki Group (Palaeontological Study of the Miyazaki Group-5). Trans, Proc. Palaeont. Soc. Japan. N.S. (31), 
253-264, 3 text-figs., 3 tables, $1 \mathrm{pl}$.

(1958-b) : Medial and Late Caenozoic History in Kyushu, Japan, with Special Reference to its Characters of Sedimentation and Tectonics. (in Japanese). Bull. Assoc. Geol. Collab. Japan (Shinseidaino Kenkyu) (28), 8-18, 4 text-figs. (1959-a): Olivid Gastropods from the Miyazaki Group (Palaeontological Study of the Miyazaki Group-6). Japan. Jour. Geol. Geogr. 30, 169-182, 4 text-figs., $1 \mathrm{pl}$.

, (1959-b): On the Palaeontological Approach to the Speciation Process of Marine Mollusca. (in Japanese with English Résumé) Earth Science, Assoc. Geol. Collab. Japan. (45), 29-36, 6 text-figs.

, $(1960-a)$ : On Some Pectinids and Venerids from the Miyazaki Group (Palaeontological Study of the Miyazaki Group-7). Mem. Fac. Sci. Kyushu Univ., Ser. D, Geol. 9, (3), 119-149, 14 text-figs., 2 tables, 3 pls.

(1960-b) : Carditids from the Miyazaki Group (Palaeontological Study of the Miyazaki Group-8). Trans. Proc. Palaeont. Soc. Japan N.S. (37), 209-222, 2 text-figs., 1 pl.

SOVERI, U. (1952): Differential Thermal Analysis of Quartery Clays of Fennoscandia. Ann. Acad. Sci. Fennicae Ser. A, III, Geol.-Geogr. (23-29), 1-103, 29 text-figs., 8 tables.

StAinforth, R. M. (1960): Current Status of Transatlantic Oligo-Miocene Correlation by Means of Planktonic Foraminifera. Revue de Micropaleontologie, 2, (4), 219-230.

Stenzel, H. B. (1949): Successional Speciation in Paleontology: The Case of the Oysters of the Sellae-formis Stock. Evolution, 3, (1), 34-50, 8 text-figs.

Sugryama, Toshio (1935): On the Variation on the Shells of Living and Fossil Umbonium from Japan and its Evolution (in Japanese). Jour. Geol. Soc. Japan, 42, (503), 404-430, 1 pl. and 503, 449-482, 28 text-figs.

Suzuki, Koichi (1932): Pliocene Beds North of Atsuki, Kanagawa Prefecture, Japan (in Japanese). Jour. Geol. Soc. Tokyo 39, (461), 49-70, 4 text-figs. and (462), 97-132.

_- (1934-a): On the Evolution of Umvonium (in Japanese). ibid. 41, (485), $67-81,4$ text-figs.

- _ , (1934-b): Supplementary Notes on the Evolutoin of Umbonium (in Japanese). ibid. 41, (491), 537-539, 1 text-fig.

Sverdrup, H. W., Johnson, M. W. and Fleming, R. S. H. (1957): The Oceans: Their Physics, Chemistry and General Biology, (7th print). Prentice-Hall, Englewood Cliff, N.J. 1-1087, 265 text-figs., 120 tables, 5 extra tables, 7 charts.

Sylvester-Bradley, P. C. (1951): The Subspecies in Palaeontology. Geol. Mag. 88, (2) , 88-102, 6 text-figs., 4 tables.

, (Ed.) (1956): The Species Concept in Palaeontology. Systematics Assoc. Publ. (2), 1-292.

TAI, Yoshirô (1959): Miocene Microbiostratigraphy of West Honshû, Japan. Jour. Sci. Hiroshima Univ. Ser. C, 2, (4), 265-395, 34 tables, 5 pls.

TAKI, Isao (1938): On the Molluscan Distribution in Japanese Waters (in Japanese). Zool. Mag. (Tokyo), 50, (4), 176-177.

Teichert, Curt (1958-a): Some Biostratigraphic Concepts. Geol. Soc. Amer. Bull. 69, (1), 99-120.

-, (1958-b) : Concepts of Facies. Amer. Assoc. Petrol. Geol. Bull. 42, (11), 2719-2744, 1 text-fig.

Tesch, P. (1920) : Jungtertiaere und Quartaere Mollusken von Timor, IIter Teil. Paläontologie von Timor, Lief. 8, 41-121, 22 pls.

THIELE, Johannes (1935): Handbuch der Systematischen Weichtierkunde, Bd. II, 
Teil 4, Vergleichende Morphologie, Phylogenie und Geographische Verbreitung. Gustav Fischer, Jena. 1023-1154, 4 text-figs.

Thoroson, Gunnar (1936): The Larval Development, Growth and Metabolism of Arctic Marine Bottom Invertebrates. Medd. om Gr申nland, (100), 1-155, 14 text-figs.

(1957): Bottom Communities. (in Treatise in Ecology and Paleoecology Vol. 1, Ecology). Geol. Soc. Amer. Mem. 67, 461-534, 20 text-figs.

TokudA, Mitoshi (1957): Evolution (rev. ed.) (in Japanese). Iwanami Science Series. (144), 1-250, 76 text-figs., 8 tables, 1 pl.

Tsuchi, Ryuichi (1955): The Palaeo-ecological Significance of the Later Pliocene Molluscan Fauna from the Kakegawa District, the Pacific Coast of Central Japan. Rep. Liberal Arts Fac., Shizuoka Univ. (Nat. Sci.) 1, (8), 45-58, 2 text-figs., 23 tables, 1 pl.

- , (1956): The Ecological Distribution of Marine Mollusca Living in the Coast of Shizuoka Prefecture, the Pacific Side of Central Japan. ibid. 1, (10), 15-24, 4 text-figs.

, (1958): A Note on Mollusca Dredged from Suruga Bay, the Pacific Side of Central Japan. ibid. 2, (2), 69-76, 3 text-figs., 6 tables, 2 pls.

-.._. (1959): Molluses and Shell Remains from the Coast of Chihama in the Sea of Enshû, the Pacific Side of Central Japan. ibid. 2, (4), 143-152, 2 text-figs., 2 tables, 1 pl.

VREdenberg, E. (1921): Results of a Revision of some Portion of Dr. NoEtLing's Second Monograph of the Tertiary Fauna of Burma. Rec. Geol. Surv. India 51, (3), 224-302, 1 text-fig.

UCHio, Takayasu (1947): Geology of the Miyazaki District (in Japanese). Jour. Geol. Soc. Japan 53, (622-627), 87-88.

UJine, Hiroshi (1959): Standard Age-Division of the Middle Tertiary by Means of Pelagic Foraminifera (Review) (in Japanese). Earth Science Assoc. Geol. Collab. Japan (42), 25-40, 6 tables.

VLERK, I. M. van der (1955) : Correlation of the Tertiary of the Far East and Europe. Micropaleontologist 1, (1), 72-75, 2 tables.

Vlerk, I. M. von der, und UMbGrove, J. H. F. (1928) : The Genus Lepidocyclina in the Far East. Eclog. Geol. Helvet. 21, 182-211, 3 tables, 18 pls.

WANNER, J. und HAHN, E. (1935) : Miocäne Mollusken aus der Landschaft Rembang. Zeitschr. Deut. Geol. Geselsch. 87, (4), 222-273, 3 text-figs., 5 pls.

Wayland, Edward James and Davies, Arthur Morley (1923): The Miocene of Ceylon. Quart. Jour. Geol. Soc. London 79, 577-602, 7 text-figs., 2 pls.

Wheeler, Harry E. (1958) : Time-Stratigraphy. Amer. Assoc. Petrol. Geol. 42, (5), 1047-1063, 7 text-figs.

Woodring, Wendel P. (1925): Contributions to the Geology and Palaeontology of the West Indies: Miocene Mollusks from Boden, Jamaica: Pelecypods and Scaphopods. Carnegie Inst. Washington Publ. (366), 1-222, 28 pls.

- , (1926): American Tertiary Mollusks of the Genus Clementia. U. S. Geol. Surv. Prof. Pap. (147-c), 25-47, 1 text-fig., 4 pls.

, (1928): Contributions to the Geology and Palaeontology of the West Indies: Miocene Mollusks from Boden, Jamaica: Pt. II, Gastropods and Discussion of Results. Carnegie Inst. Washington Publ. 385, 1-539, 40 pls.

Yokoyama, Matajiro (1923): On Some Fossil Mollusca from the Neogene of Izumo. Japan. Jour. Geol. Geogr. 2, (1), 1-9, 2 pls.

, (1926-a): Tertiary Mollusca from Southern Totomi. Jour. Fac. Sci. Imp. Univ. Tokyo Sect. 2, 1, (9), 313-364, 4 pls.

, (1926-b) : Tertiary Shells from Tosa. ibid. 1, (9), 365-368, 1 pl. 
, (1928): Pliocene Shells from Hyuga. ibid. 2, (7), 331-350, 2 pls.

- _ , (1929-a): Mollusca from the Oil-Field of the Island of Taiwan. Imp. Geol. Surv. Japan Rep. (101), 1-112, 18 pls.

, (1929-b): Pliocene Shells from Tonohama. ibid. (104), 9-17, 2 pls.

Zhemchuzhinikov, Y. A. (1959): Nomenclature of Facies. Intern. Geol. Rev. 1, (1), 65-72. 
Plate 1. Geological map of the Miyazaki district. Locality numbers are same to those in the text (pp. 99-117)
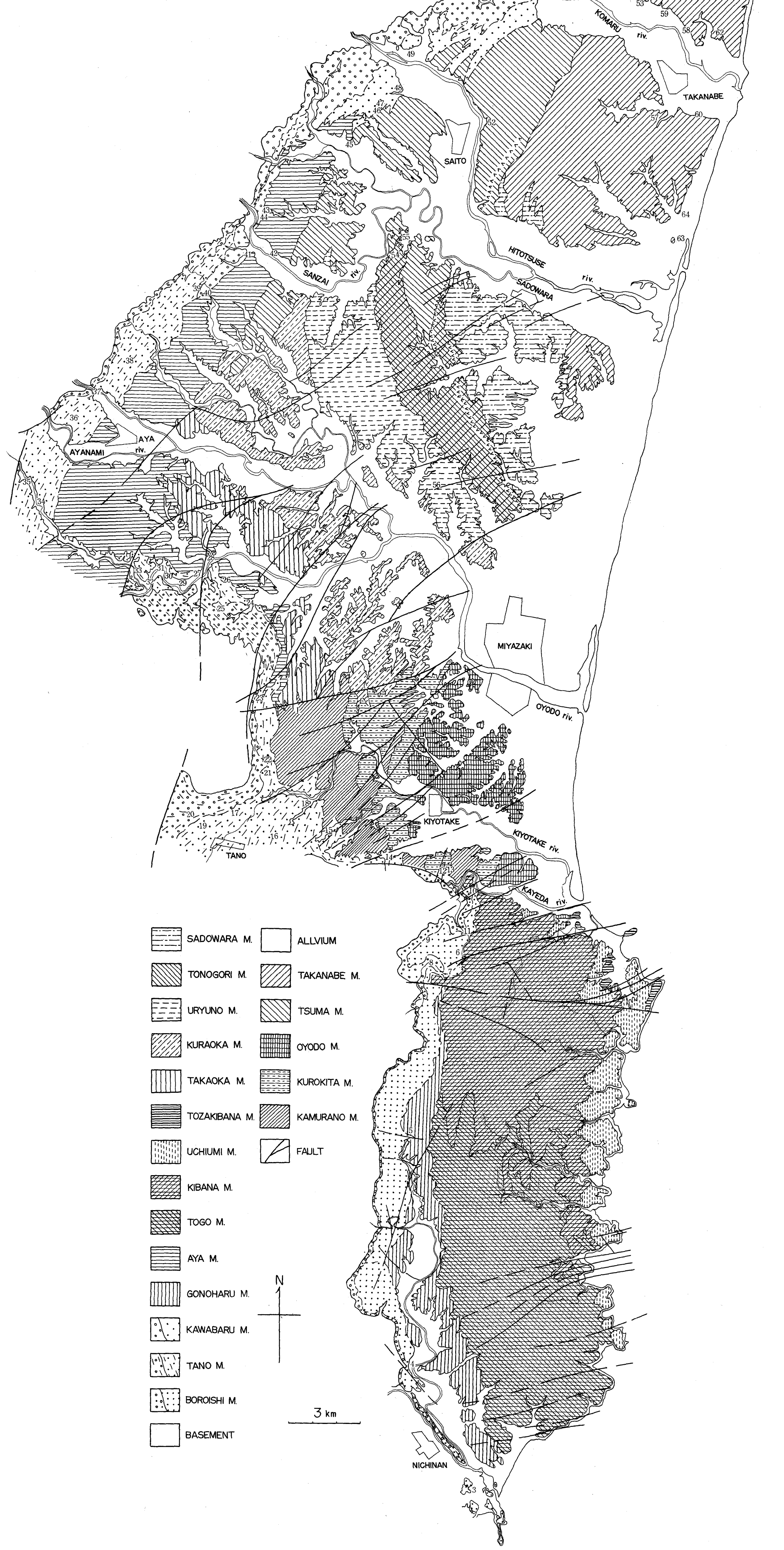


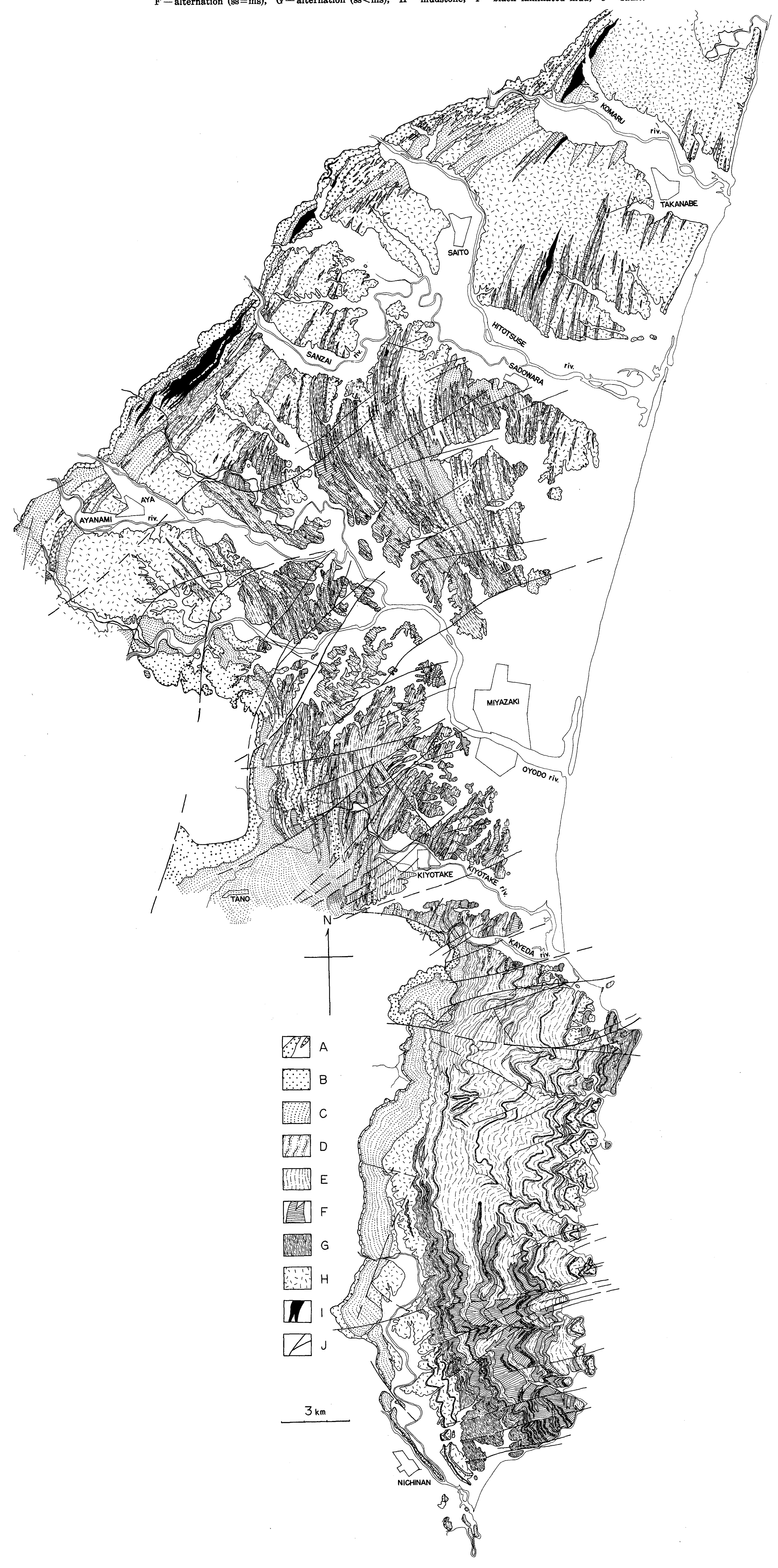




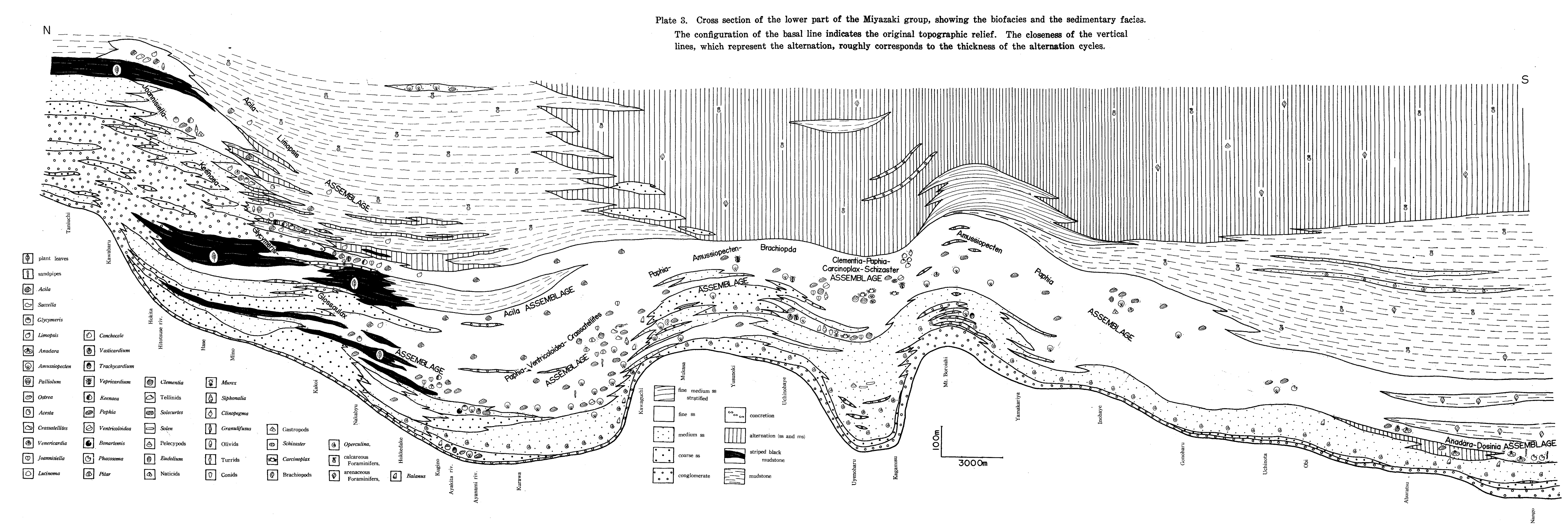

